\title{
Mitochondria in a personalized cancer treatment approach
}

Citation for published version (APA):

van Gisbergen, M. W. (2017). Mitochondria in a personalized cancer treatment approach. [Doctoral Thesis, Maastricht University]. Datawyse / Universitaire Pers Maastricht. https://doi.org/10.26481/dis.20170329mvg

Document status and date:

Published: 01/01/2017

DOI:

10.26481/dis.20170329mvg

Document Version:

Publisher's PDF, also known as Version of record

Document license:

Unspecified

\section{Please check the document version of this publication:}

- A submitted manuscript is the version of the article upon submission and before peer-review. There can be important differences between the submitted version and the official published version of record.

People interested in the research are advised to contact the author for the final version of the publication, or visit the DOI to the publisher's website.

- The final author version and the galley proof are versions of the publication after peer review.

- The final published version features the final layout of the paper including the volume, issue and page numbers.

Link to publication

\footnotetext{
General rights rights.

- You may freely distribute the URL identifying the publication in the public portal. please follow below link for the End User Agreement:

www.umlib.nl/taverne-license

Take down policy

If you believe that this document breaches copyright please contact us at:

repository@maastrichtuniversity.nl

providing details and we will investigate your claim.
}

Copyright and moral rights for the publications made accessible in the public portal are retained by the authors and/or other copyright owners and it is a condition of accessing publications that users recognise and abide by the legal requirements associated with these

- Users may download and print one copy of any publication from the public portal for the purpose of private study or research.

- You may not further distribute the material or use it for any profit-making activity or commercial gain

If the publication is distributed under the terms of Article $25 \mathrm{fa}$ of the Dutch Copyright Act, indicated by the "Taverne" license above, 


\section{Mitochondria in a personalized cancer treatment approach}




\section{Cover}

Cover design: Anna Karlström

\section{Production}

Printing: Datawyse | Universitaire Pers Maastricht ISBN 9789461596772

(c) Copyright: Marike W. van Gisbergen, Maastricht, 2017.

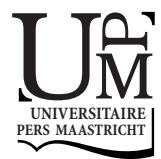




\title{
Mitochondria in a personalized cancer treatment approach
}

\author{
Proefschrift \\ ter verkrijging van de graad van doctor aan de Universiteit Maastricht, \\ op gezag van de Rector Magnificus, Prof. dr. Rianne M. Letschert, \\ volgens het besluit van het College van Decanen \\ in het openbaar te verdedigen \\ op woensdag 29 maart 2017 om 14:00 uur \\ door
}

Marike Willemijn van Gisbergen 


\section{Promotor}

Prof. dr. P. Lambin

\section{Copromotores}

Dr. L.J. Dubois

Dr. A.M. Voets, Universiteit Hasselt

\section{Beoordelingscommissie}

Prof. dr. F.C.S. Ramaekers (voorzitter)

Prof. dr. R.P. Coppes, UMC Groningen

Dr. P. Willems, Radboud UMC Nijmegen

Prof. dr. L.P.A.J. Schrauwen

Dr. F. van Tienen 


\section{Contents}

Chapter 1 General introduction and thesis outline

Chapter 2 How do changes in the mtDNA and mitochondrial dysfunction influence cancer and cancer therapy? Challenges, opportunities and models

Chapter 3 Distinct radiation responses after in vitro mtDNA depletion are potentially related to oxidative stress

Chapter 4 Mitochondrial dysfunction inhibits hypoxia-induced HIF-1 $\alpha$ stabilization and expression of downstream targets

Chapter 5 New approach of delivering cytotoxic drugs towards CAIX expressing cells: A concept of dual-target drugs

Chapter 6 Nitroglycerin's influence on tumor hypoxia and perfusion in nonsmall cell lung cancer: a window-of-opportunity trial

Chapter 7 Chemical reactivity window determines prodrug efficiency toward glutathione transferase overexpressing cancer cells

Chapter 8 General discussion

Summary

Samenvatting

Valorization Addendum

Acknowledgements / Dankwoord

Curriculum Vitae 255

List of publications 

Chapter

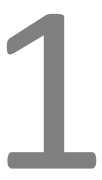

General introduction and thesis outline 



\section{General introduction}

Now-a-days cancer is one of the leading causes of death worldwide. In the Netherlands alone already 43.214 deaths from cancer were registered for the year 2014 [1]. Cancer is a generic term for many types of diseases, which all involve several common characteristics and is used for both benign and malignant tumors. A cell mass that is rapidly dividing and could potentially invade its surrounding tissues is often referred to as a cancer. The abnormal growth of this cell population starts by sequential accumulation of different DNA mutations inherited by cell division. Under normal physiological conditions tissues have the capability to replace mutated cells. However, when the normal cellular functions are compromised a benign tumor mass could arise. Cells from a benign tumor are able to evade apoptosis; however, they don't have the capability to invade other tissues yet. When a cell population acquires additional mutations, the rise of a malignant tumor may occur. Tumors display several characteristics which enable the tumorigenic and/or metastatic potential of the cell population and include sustained proliferative signaling, evading growth suppressors, avoiding destruction by the immune system, enabling replicative immortality, tumor promoting inflammation, invasion and metastatic potential, inducing angiogenesis, genomic instability and genomic mutations, resisting cell death and deregulated cellular energetics. Often these characteristics are referred to as the 'Hallmarks of cancer', which have been comprehensively described by Hanahan and Weinberg [2, 3].

Treatment of malignancies is of great importance in order to increase survival and quality of life of cancer patients. Now-a-days many cancer treatment options are available in the clinic although the majority of the patients are currently still treated by surgery, chemotherapy or radiotherapy, or a combination of those. The choice of therapy is dependent on several factors such as the patient's condition, the tumor's histopathology, stage and location. Different cancer types often do benefit from additional hormone therapy [4, 5] or even immunotherapy [6]. Heterogeneity amongst patients, but also amongst tumors and their microenvironment influence treatment outcome. Also, heterogeneities in nutrient supply, proliferation, $\mathrm{pH}$ levels and oxygen supply or consumption influence the treatment response. Knowledge of a tumor's status and the predictive power of treatment response could therefore improve treatment efficacy and reduce treatment costs [7].

\section{The role of the tumor microenvironment in tumor survival}

The tumor and its microenvironment (TME) is a very complex but important system, from which our understanding is gradually increasing. The TME consists out of blood vessels, signaling molecules, the extracellular matrix and many cell types such as 
fibroblasts, dendritic cells, lymphocytes and macrophages [8]. The TME is a heterogeneous, dynamic mechanism of various cells, all having a different gene expression profile, metabolism and oxygen supply. The lack of nutrient and oxygen supply interacts with two main characteristics of a malignancy, its bioenergetics and angiogenesis. Hypoxia can occur through different mechanisms. Diffusion-limited hypoxia arises in cells with an oxygen consumption higher than its supply or beyond the oxygen diffusion distance. This phenomenon is commonly referred to as chronic hypoxia [9]. Perfusion-limited or acute hypoxia arises around vessels which are temporarily closed, frequently caused by the malformation of the blood vessels in a tumor [9]. In order to survive, malignant cells often switch to a more glycolytic phenotype, independent whether or not oxygen is present, a phenomenon better known as the Warburg effect [10] (Fig. 1), resulting in acidification of the extracellular environment. The enhanced glycolysis produces massive amounts of lactate in the cytoplasm, contributing together with production of $\mathrm{CO}_{2}$ to an acidic intracellular $\mathrm{pH}$. In order to survive, cancer cells correct this intracellular acidification by the increased expression and activity of certain $\mathrm{pH}$ regulating membrane transporters regulating lactate efflux (monocarboxylate transporters (MCT4)) and Na-driven proton extrusion (sodium proton exchangers (NHE) and anion exchangers AE1 and AE2) [11].

\section{Proliferative and differentiated tissues}

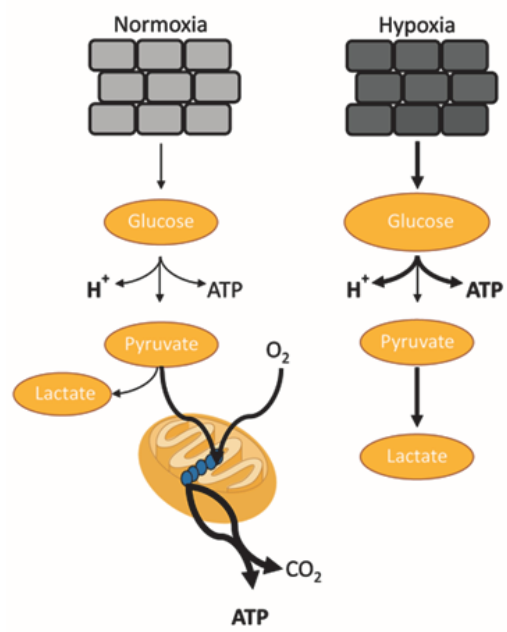

\section{Tumorigenic tissues}

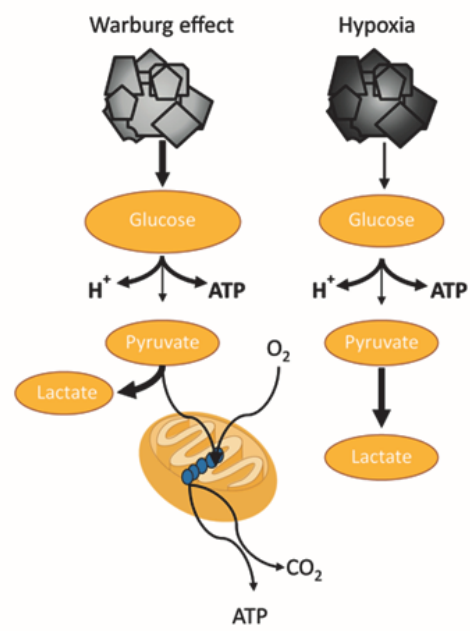

Figure 1. Overview of the influence of hypoxia in the metabolism of proliferative and differentiated tissues and tumorigenic tissues.

Adapted from [10].

Another group of important enzymes for maintaining the intracellular $\mathrm{pH}$ are the carbonic anhydrases (CAs). CAIX, a transmembrane protein with its active site 
extracellularly, is one of the CAs often found to be upregulated in cancer leading to worse locoregional control, disease progression, and even leads to a higher risk to develop metastasis in patients [12]. CAIX catalyzes the conversion of carbon dioxide $\left(\mathrm{CO}_{2}\right)$ and $\mathrm{H}_{2} \mathrm{O}$ into bicarbonate and a proton $\left(\mathrm{H}^{+}\right)[13] . \mathrm{CO}_{2}$ is an acid produced as a byproduct at the tricarboxylic acid (TCA) cycle and when pyruvate is converted into acetylCoA. $\mathrm{CO}_{2}$ can also be produced as the result of the reduction of bicarbonate by intracellular CAs such as CAll. $\mathrm{CO}_{2}$ diffuses across the cell membrane and forms the substrate for rehydratation by either CAIX or CAXII to produce bicarbonate (Fig. 2). To sustain an alkaline intracellular $\mathrm{pH}$, cells enable transport of bicarbonate via bicarbonate transporters or anion exchangers into the cell. This complex system results in acidification of the extracellular environment and is a mechanism often observed in tumors to promote survival [11].

CAIX expression is closely regulated by hypoxia as it contains a hypoxia response element in its promotor region and therefore is a direct downstream target of hypoxia inducible factor 1 alpha (HIF-1 $\alpha$ ) activity [14] (Fig. 2.) Under normal oxygen tension (Fig. 2A), HIF-1 $\alpha$ is hydroxylated at its proline residues by prolyl hydroxylases (PHDs), which targets HIF-1 $\alpha$ for proteasomal degradation through binding with the Von Hippel-Lindau complex (VHL) leading to ubiquitylation via E3 ubiquitin-protein ligases. However, under conditions of hypoxia (Fig. 2A), causing downregulation of PHDs activity, or by mutations in the tumor suppressor gene VHL (mainly reported in renal cell carcinomas), HIF-1 $\alpha$ is stabilized and interaction with HIF-1ß (also known as aryl hydrocarbon receptor nuclear translocator ARNT) and translocation to the nucleus occurs. Together with cofactors such as CBP/p300 and the DNA polymerase II complex, the HIF-1 complex can bind to hypoxia response elements (HREs). HIF-1 $\alpha$ regulates specific regulatory sequences in the promotor region of target genes in order to let them be controlled by changes in oxygen concentrations (Fig. 2B). Where angiogenesis is for instance influenced by VEGF expression, metabolism is influenced by GLUT1 expression, and regulation of the extracellular pH by CAIX; all of them target genes of HIF-1 $\alpha[9,15]$. 


\section{A}

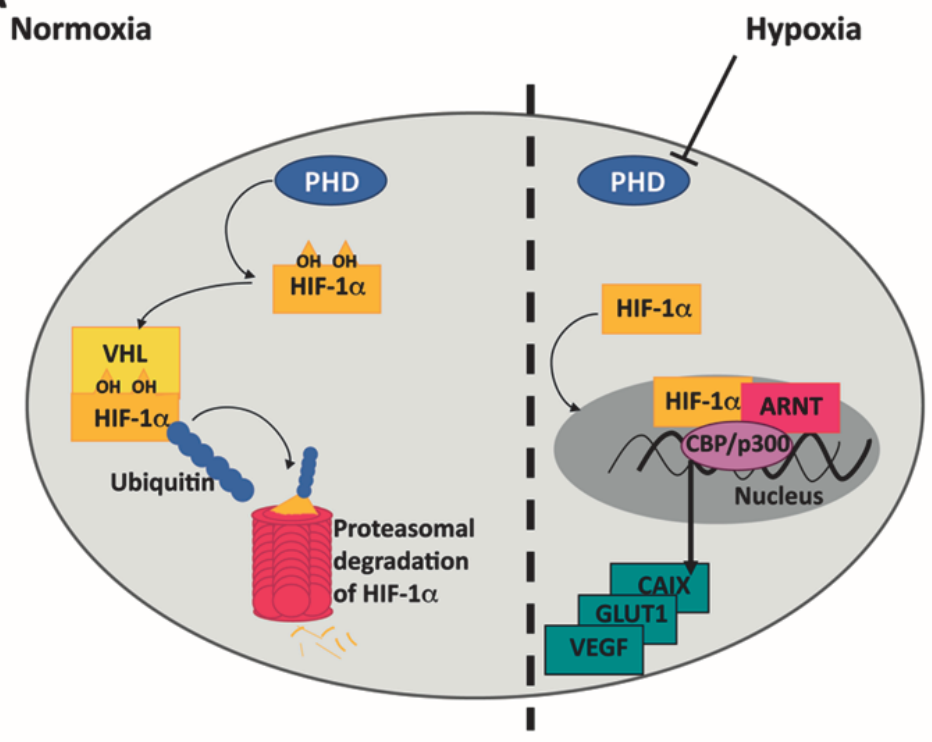

Mechanisms to maintain $\mathrm{pH}$ homeostasis under hypoxia

\section{Glucose}

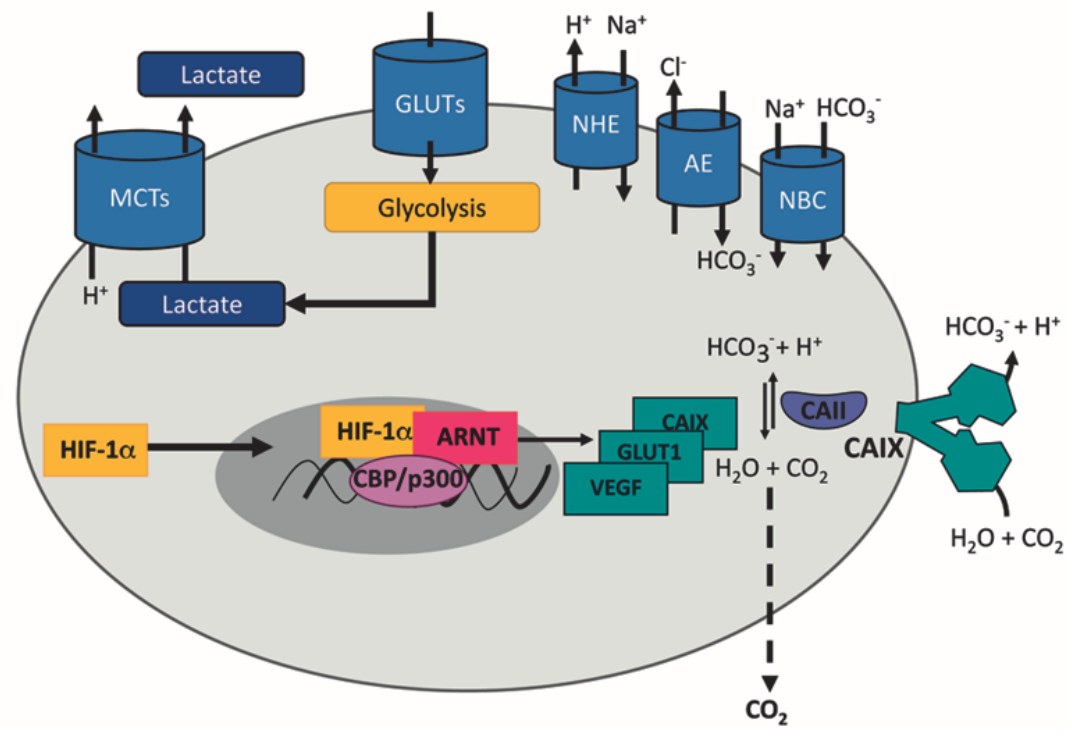

Figure 2. Overview of the influence of normoxia and hypoxia on the HIF pathway and its effects on its downstream targets.

A. HIF-1 $\alpha$ pathway under normoxic and hypoxic conditions B. The influence of HIF-1 $\alpha$ under hypoxic conditions on CAIX. ARNT (Aryl hydrocarbon receptor nuclear translocator), CAll (carbonic anhydrase II), CAIX (carbonic anhydrase IX), GLUT1 (glucose transporter 1), HIF-1 $\alpha$ (Hypoxia Inducible Factor $1 \alpha$ ), MCTs (monocarboxylate transporters), NHE ( $\mathrm{Na}+\mathrm{H}+$ exchangers) AE (anion exchangers) NBC (sodium-bicarbonate co-transporter), VEGF (vascular endothelial growth factor). Adapted from [16, 17]. 


\section{Mitochondria, ROS production and its role in the tumor microenvironment}

Mitochondria are organelles of $0.5 \sim 3 \mu \mathrm{m}$ in size located in the cytoplasm of the cell. They can fuse with each other (fusion) and be separated again (fission) and thereby influence their function, their formed network and their own genetic material (mitochondrial DNA; mtDNA). Mitochondria are well known for their role in the production of ATP and are therefore often referred to as the power plant of a cell. Furthermore, they also have their own toxic waste production better known as reactive oxygen species (ROS). ROS plays an important role in maintaining the cellular redox status, but also contributes to enzymatic activity and intracellular signaling [18-20]. Mitochondria consist of a double-layered membrane, creating two compartments, the internal matrix and intermembrane space. Although both outer and inner membrane contains transport proteins, only the inner membrane harbors subunits of the electron transport chain. The matrix contains enzymes that metabolize pyruvate or fatty acids in order to produce acetyl CoA as well as enzymes that oxidize acetyl CoA further in the Kreb's cycle [21]. Every cell itself can contain multiple copies of mitochondria and every mitochondrion contains multiple copies of mtDNA, depending on the energy demand of the respective cell.

Under normal physiological circumstances, mitochondria use most of the oxygen present in the tissue to produce ATP. However when oxygen tension is low either a metabolic switch to glycolysis occurs [22] or cell death due to insufficient ATP production [23]. Mitochondria are known for their maternally inherited DNA encoding for 13 of the approximately 90 structural subunits of the oxidative phosphorylation chain (OXPHOS) $[24,25]$ and variations in the mtDNA can contribute to an altered energy supply in (tumor) cells $[26,27]$. It also has been reported that severe mitochondrial dysfunction impairs tumor formation. However, mild mitochondrial dysfunction can result in tumor formation [28]. This suggests that the role of mtDNA variations and its subsequently dysfunctional OXPHOS system in tumorigenicity is a complex balance.

The OXPHOS system is one of the major contributors to the production of ROS (Fig. 3), with complex I (CI) and complex III (CIII) acting as the major ROS producing sites [29-31]. $\mathrm{Cl}$ (and $\mathrm{CII}$ ) releases the produced ROS directly into the mitochondrial matrix, whereas $\mathrm{CIII}$ can release ROS either in the mitochondrial matrix or in the intermembrane space [32]. mtDNA variations can lead to dysfunction of OXPHOS resulting in an increased ROS production [33]. Also, other endogenous mechanisms (e.g. endoplasmic reticulum, peroxisomes and enzyme systems such as NADPH oxidases, nitric oxide synthase etc. [34]) or exogenous inducers (like xenobiotics and radiation [34, 35]) are contributing to cellular ROS production. In order to maintain the balance between ROS production, 
which is also used as a signaling molecule in cells, and its harmful consequences when ROS is produced in excess, cells harbor specific defense mechanisms. The cellular antioxidant mechanism protects cells from the harmful ROS effects, such as damage towards lipids, proteins and nucleic acids and the induction of double stranded DNA breaks [36]. Superoxide produced in the mitochondria is transformed into hydrogen peroxide $\left(\mathrm{H}_{2} \mathrm{O}_{2}\right)$ by superoxide dismutases either in the mitochondria themselves by the manganese superoxide dismutase (MnSOD/SOD2) or in the nucleus, lysosomes or cytosol by the copper-zinc superoxide dismutase (CuZnSOD/SOD1). Catalases, located at the peroxisomes, are responsible for the subsequent conversion from $\mathrm{H}_{2} \mathrm{O}_{2}$ towards oxygen $\left(\mathrm{O}_{2}\right)$ and water $\left(\mathrm{H}_{2} \mathrm{O}\right)$. In tumors, the efficacy of these defense mechanisms is usually sub-optimal due to deregulated or even exhausted antioxidant systems leading to an increased metastatic potential. For example, it has been shown that mitochondrial superoxide plays a role in cellular migration, invasion and clonogenic potential [37].

The thioredoxin system (Fig. 3) consisting out of thioredoxin peroxidases (peroxiredoxins), thioredoxin reductases and its main substrate thioredoxin ( $\operatorname{Trx}$ ), is of great importance in catalyzing the reaction of $\mathrm{H}_{2} \mathrm{O}_{2}$ into $\mathrm{H}_{2} \mathrm{O}$ under the influence of NADPH. Mitochondria even have their own specific thioredoxin (Trx2), thioredoxin reductase (TrxR2) and peroxiredoxin ( $\operatorname{Prx} 3)$ [38]. Where thioredoxin is involved in redox regulation and can act as an antioxidant, thioredoxin peroxidases are involved in the regulation and reduction of peroxides [38, 39]. Also, glutathione peroxidases are able to catalyze the reduction of $\mathrm{H}_{2} \mathrm{O}_{2}$ using the substrate glutathione, a very important antioxidant [38]. Glutathione is a low molecular weight non-protein thiol, which is very abundant in the cell and especially in the mitochondria. It is mostly present in its reduced form (GSH) but can be oxidized into GSSG. To maintain the cellular redox homeostasis, GSSG can be reduced by glutathione reductases and serves therefore as an antioxidant defense mechanism. Studies have shown that an interplay between the thioredoxin and the glutathione system exists [40,41]. Also, these systems could be potentially interesting to target in a prodrug-based approach. Other potential targets could be glutathione S-transferases (GSTs), a group of enzymes that use glutathione as a co-factor and catalyze the conjugation of glutathione to a wide range of endogenous and exogenous electrophilic compounds. Mitochondrial (GST Kappa), cytosolic (GST Alpha, mu, Pi, Sigma, Theta, Omega and Zeta) and microsomal (MAPEG family), as well as multiple classes of GSTs have been reported [42]. Overexpression is often reported in tumors and can contribute to a drug resistant phenotype as GSTs serve Phase II detoxification enzymes and are involved in the regulation of oxidative stress [43]. 


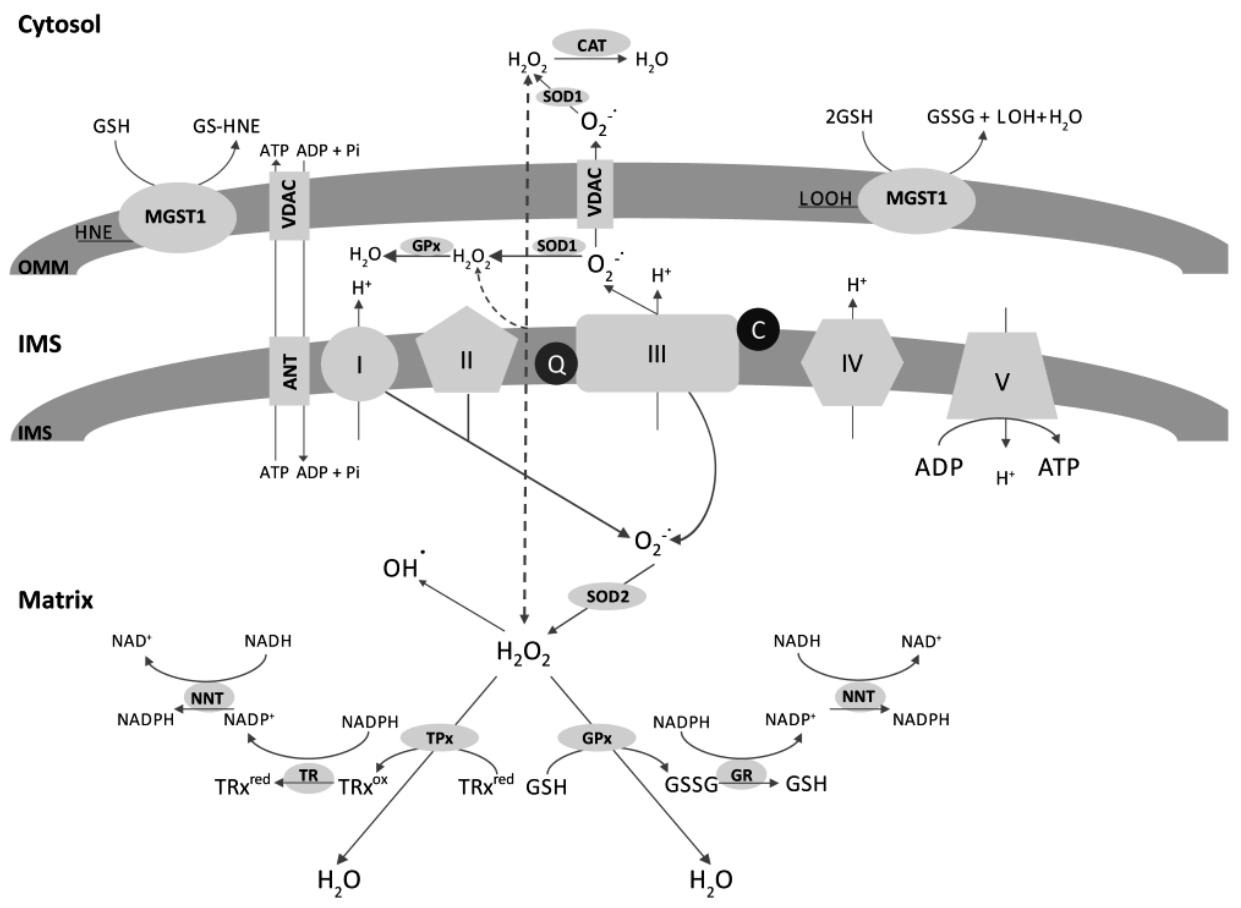

Figure 3. Schematic representation of the mitochondrial antioxidant system.

I-V, OXPHOS complex I-V; ANT, adenine nucleotide translocases; CAT, catalase; GSH, reduced glutathione; GSSG, oxidized glutathione; GPx, glutathione peroxidase; GR, glutathione reductase; 4-hydroxynonenal, HNE; IMM, inner membrane space; LOH, lipid alcohol; MGST1, microsomal glutathione transferase; NNT; nicotinamide nucleotide transhydrogenase; OMM, outer membrane space; SOD, superoxide dismutase; TPX, thioredoxin peroxidase; TR, thioredoxin reductase; $\mathrm{TRx}^{\mathrm{red}} / \mathrm{TRx}^{\mathrm{ox}}$; reduced and oxidized thioredoxin; VDAC, voltage-dependent anion-selective channel. Adapted from [44-47].

For instance, one of the MAPEG GSTs members MGST1 is highly abundant in the outer membrane of the mitochondria and in the membrane of the endoplasmic reticulum, expressed in most tissues with the highest abundance in liver tissue. MGST1 overexpression has been reported in various tumor types ranging from acute myeloid leukemia, colorectal cancer to Ewing's sarcoma [48]. MGST1 is able to act as both a Glutathione peroxidase and GST [48]. It can protect against induced lipid peroxidation and oxidative stress $[49,50]$ but also against doxorubicin and cisplatin exposure causing a drug resistance phenotype [51]. In Ewing's sarcoma patients increased expression of MGST1 was found to influence prognosis via resistance against doxorubicin [52]. Potentially this could be counteracted by a prodrug approach were the cytotoxic agent is only released in a GST dependent manner $[51,53]$.

Upon insufficient oxygen supply, tumors shift their metabolism to an anaerobic glycolysis phenotype. It is however debatable what happens with the ROS production under these low oxygen conditions. It has been suggested that non-specific ROS is reduced [54]. 
Conversely, specifically $\mathrm{CII}$ and $\mathrm{CIII}$ mediated ROS production is increased under low oxygen tensions [55-57]. Additionally it has been suggested that only CIII specific ROS contributes to HIF-1 $\alpha$ stabilization by inhibiting PHDs [56, 58, 59]. The exact role of other mitochondrial ROS sources remains unknown. Mitochondrial ROS potentially could act as a signaling molecule contributing to tumorigenesis, tumor progression and metastasis formation [37, 60-63]. However, reducing ROS levels could be a double-edged sword as it also has been reported to stimulate tumor progression [64].

\section{The unfavorable treatment response in cancer patients}

Treatment resistance occurs in a substantial part of cancer patients. Resistance to chemotherapeutic agents is a major problem in cancer treatment and can be attributed 1 ) to the genetics of the tumor, i.e. mutations in genes regulating apoptosis, cell cycle and DNA-repair, 2) to overexpression of drug detoxification enzymes like glutathione transferases and 3) to changes in membrane barriers such as influx or efflux pumps [43, 65]. This can cause recurrence of the tumor and even can be fatal to the patient. The drug resistance can already exist at the moment the tumor arises or can be acquired during treatment. For instance drug resistance towards doxorubicin, a commonly used cytostatic drug for leukemia and (soft tissue) carcinomas, has been reported resulting in an unfavorable therapeutic outcome [66]. Additionally, low oxygen regions (hypoxia) heterogeneously spread in tumors can also lead to a drug resistance phenotype as a result of decreased drug availability or lower uptake of certain drugs due to the increased extracellular acidification $[67,68]$ and has been reported for e.g. doxorubicin, etoposide, cisplatin, 5-fluorouracil, gemcitabine, and docetaxel [69-74]. Furthermore, resistance to radiotherapy (RT) is a well-known phenomenon in cancer patients [75-77]. Cells with altered DNA-repair systems or hypoxic regions can contribute to this resistant phenotype. To be effective as therapy according to the oxygen-fixation theory, RT needs oxygen to "fix" the DNA damage, i.e. to convert a reactive hydroxyl radical in a chemically stable carboxyl group [35]. When less oxygen is available, i.e. an increased hypoxic fraction, the radiotherapy efficacy decreases leading to a worse prognosis in e.g. head and neck cancer patients [76]. The success of cancer treatment is therefore not only dependent on the selected therapy, but is also influenced by the tumor microenvironment. 


\section{Repurpose drug approach to decrease tumor hypoxia and improve treatment outcome.}

Repurposing already well-known drugs and use their therapeutic potential is a valid approach to improve the standard treatment of cancer patients and simultaneously reduce drug development costs and thereby treatment costs. One of those potential interesting drugs for repurposing is metformin. Metformin, a biguanide often used in type 2 diabetes treatment, has been linked to a reduced risk of developing cancer in this patient population [78]. Another fascinating feature of metformin is its potential use as a metabolism-changing agent. Metformin can reduce $\mathrm{Cl}$ activity and thereby decreases both the ATP production and oxygen consumption of the OXPHOS system [79]. As a result, levels of adenosine monophosphate (AMP) increase thereby triggering an increase in AMP activated kinase (AMPK) levels in the cell [80].

Metformin can also reduce gluconeogenesis (generation of glucose from noncarbohydrate carbonic substrates such as lipids) through interference with the mitochondrial glycerophosphate dehydrogenase (mGPD) enzyme [81]. Furthermore, also the metformin-induced increased AMPK levels and diminished glucagon signaling (via decreased cyclic AMP levels) contribute to a decreased gluconeogenesis [82]. In cancer patients, metformin probably results in decreased proliferation rates as a consequence of the combination of the above-described mechanisms and consequent reduction of glucose and increase in insulin sensitivity. In addition, metformin is able to decrease the hypoxic fraction in tumors, probably based on the principle that by using less oxygen via respiration, the oxygen can diffuse further into the tumor. Both in vitro and in vivo studies already showed positive effects for the combination of RT with metformin $[83,84]$. Also in small clinical trials, it was observed that therapeutic response rates were indeed improved among different tumor types [85-87]. However, in a breast cancer patient cohort more interruptions from their radiation therapy schedules were observed in the metformin group due to radiation dermatitis [88] (for a comprehensive review see [98]). In order to increase therapeutic efficiency, recently a mitochondrial targeted metformin compound, mito-metformin, has been developed. Pre-clinical studies showed that ROS production was increased while tumor growth and progression was reduced in human pancreatic ductal adenocarcinoma xenografts, suggesting that the functionality of metformin could still be further improved [89]. Currently, phenformin, another more potent biguanide drug is making its comeback. Where phenformin was withdrawn from the North-American market in 1977 by the FDA due to fatal lactic acidosis in diabetic patients, it currently regained interest. Although, no clinical studies have been published with phenformin investigating the potential usage of phenformin in cancer patients, pre-clinical studies did show promising results on tumor growth delay and enhanced therapeutic effects in combination treatments [90-92]. It has been suggested that phenformin has less toxicity compared to the most 
commonly used chemotherapeutics, making it potentially more acceptable for cancer treatment compared to diabetes [91].

Another potential candidate for a drug repurposing approach is nitroglycerin. Although the public knows nitroglycerin as one of the key components for dynamite it is not only used as an explosive. Currently, it is also a very common drug for heart diseases such as angina pectoris due to its nitric oxide releasing properties. Those released nitric oxides cause a vasodilatation effect in the affected heart tissue, and thereby increase the blood flow in cardiac patients. Those properties also make it an interesting drug for its use in cancer patients. It had been suggested that nitric oxides could influence the hypoxia status of the tumors by increasing the tumor perfusion as they can serve as vasodilators. This might result in a potentially improved cancer treatment by enhanced chemotherapy delivery or by influencing the radiosensitization of tumors [93, 94]. Other potential interesting mechanisms are increased formation of intracellular free radicals caused by the nitric oxide donors.

\section{Objective and thesis outline}

The aim of this thesis was to investigate the role of mitochondrial function, reactive oxygen species (ROS) and hypoxia modulation in tumors in view of the possible implementation in a personalized treatment approach in order to reduce the possibility of recurrences or adverse effects.

A comprehensive and detailed overview on the literature involving mitochondrial dysfunction and mitochondrial DNA variations influencing cancer and cancer treatment is given in chapter 2. Mitochondria and radiotherapy share several molecular processes like ROS production. Mitochondria are important for ATP production and the regulation of apoptosis [95], whereas ionizing radiation induces ROS formation which can lead to cell death [96]. It is known that exposure to ionizing radiation can up-regulate mitochondrial content and function [97], and ATP is required to repair radiation induced damages. Several molecular approaches have been used to investigate the effect of energetic stress and/or stress inducers (irradiation and hypoxia) in relation to mitochondrial function. In chapter 3 a proof-of-principle study was conducted to investigate the influence of mtDNA variations on RT response both in immortalized bronchial cells as in tumor cell lines. We used complete mtDNA depletion in our in vitro system as an extreme model of mtDNA-related mitochondrial dysfunction. In addition, the potential role of ROS and antioxidants in this response has been investigated.

Stabilized HIF-1 $\alpha$ contributes to a glycolytic phenotype of the tumor. However, under re-oxygenation, this glycolytic phenotype (Warburg phenotype) persists and is associated with a decreased intracellular $\mathrm{pH}$. This induced Warburg phenotype could be 
potentially caused by mtDNA variations, which makes it relevant to investigate the effect of changes in the function of Complex I. Therefore, in chapter 4 we investigate the effect of complex I inhibition on HIF-1 $\alpha$ function and downstream targets under hypoxic conditions using the anti-diabetic drug metformin, rotenone or by genetically modifying mtDNA-encoded complex I genes, via the usage of cytoplasmic hybrid and mtDNA depletion models.

In chapter 5 we investigated specific targeting of the biguanide phenformin (more potent than metformin) to tumor cells, in order to reduce the risk of phenformininduced lactic acidosis in normal tissue. Therefore, we exploited the tumor-specific expression of the hypoxia-regulated enzyme CAIX. We have designed a series of CAIX targeting compounds using the dual-drug approach where the CAIX active site targeting sulfonamide moiety is coupled to several anti-cancer drugs and evaluated these for their therapeutic efficacy. Specifically, the sulfonamide-phenformin was investigated for its inhibitory properties on mitochondrial respiration.

Repurposing FDA approved drugs might be another approach to treat cancer since their side effects are known. This will reduce drug development costs and thereby treatment costs in order to keep cancer treatment affordable while improving cancer treatment. Nitroglycerin is a nitric oxide donor and used as vasodilator for angina pectoris. Vasodilating agents offer the opportunity to counteract tumor hypoxia by opening temporarily occluded vessels thereby increasing blood flow. Using a window-ofopportunity study design, we investigated in chapter 6 the possibility of using nitroglycerin to reduce tumor hypoxia in NSCLC patients and furthermore, we assessed the underlying molecular effects such as the potential inhibitory effect on mitochondrial respiration as possible explanation for a decrease in tumor hypoxia.

Another approach to increase therapeutic efficacy is described in chapter 7, in which we investigated specifically targeting glutathione S-transferases (GSTs), a phase II detoxification enzyme family. GST overexpression has been reported in many cancer types and plays an important role in drug resistance towards anti-cancer drugs and oxidative stress response. Therefore, GST targeting pro-drugs were developed. Doxorubicin derivatives have been developed and evaluated, for their specifically interaction with GSTs.

Finally, in chapter 8 a general discussion on the implementation of influencing the hypoxic fractions and the role of mitochondria in respect to the metabolism of the tumor is included. Important findings and future perspectives are being discussed. 


\section{References}

1. Nederlandse Kankerregistratie. webpage cited: 2016-09-30; Available from: http://www.cijfersoverkanker.nl.

2. Hanahan, D. and R.A. Weinberg, The hallmarks of cancer. Cell, 2000. 100(1): p. 57-70.

3. Hanahan, D. and R.A. Weinberg, Hallmarks of cancer: the next generation. Cell, 2011. 144(5): p. 646-74.

4. Group, E.B.C.T.C., Effects of chemotherapy and hormonal therapy for early breast cancer on recurrence and 15-year survival: an overview of the randomised trials. Lancet, 2005. 365(9472): p. 1687-717.

5. Messing, E.M., et al., Immediate hormonal therapy compared with observation after radical prostatectomy and pelvic lymphadenectomy in men with node-positive prostate cancer. The New England journal of medicine, 1999. 341(24): p. 1781-8.

6. Atkins, M.B., et al., High-dose recombinant interleukin 2 therapy for patients with metastatic melanoma: analysis of 270 patients treated between 1985 and 1993. Journal of clinical oncology : official journal of the American Society of Clinical Oncology, 1999. 17(7): p. 2105-16.

7. Gazouli, M. and K. Souliotis, The economic considerations and implications of the stratification of future oncology therapeutics. Molecular diagnosis \& therapy, 2014. 18(4): p. 403-8.

8. Quail, D.F. and J.A. Joyce, Microenvironmental regulation of tumor progression and metastasis. Nature medicine, 2013. 19(11): p. 1423-37.

9. Harris, A.L., Hypoxia--a key regulatory factor in tumour growth. Nature reviews. Cancer, 2002. 2(1): p. 38-47.

10. Vander Heiden, M.G., L.C. Cantley, and C.B. Thompson, Understanding the Warburg effect: the metabolic requirements of cell proliferation. Science, 2009. 324(5930): p. 1029-33.

11. Damaghi, M., J.W. Wojtkowiak, and R.J. Gillies, $\mathrm{pH}$ sensing and regulation in cancer. Frontiers in physiology, 2013. 4: p. 370.

12. van Kuijk, S.J., et al., Prognostic Significance of Carbonic Anhydrase IX Expression in Cancer Patients: A Meta-Analysis. Frontiers in oncology, 2016. 6: p. 69.

13. Swietach, P., et al., The chemistry, physiology and pathology of $\mathrm{pH}$ in cancer. Philosophical transactions of the Royal Society of London. Series B, Biological sciences, 2014. 369(1638): p. 20130099.

14. Kaluz, S., et al., Transcriptional control of the tumor-and hypoxia-marker carbonic anhydrase 9: A one transcription factor (HIF-1) show? Biochimica et biophysica acta, 2009. 1795(2): p. 162-72.

15. Rankin, E.B. and A.J. Giaccia, Hypoxic control of metastasis. Science, 2016. 352(6282): p. 175-80.

16. Maes, C., G. Carmeliet, and E. Schipani, Hypoxia-driven pathways in bone development, regeneration and disease. Nature reviews. Rheumatology, 2012. 8(6): p. 358-66.

17. Neri, D. and C.T. Supuran, Interfering with $\mathrm{pH}$ regulation in tumours as a therapeutic strategy. Nature reviews. Drug discovery, 2011. 10(10): p. 767-77.

18. Oktyabrsky, O.N. and G.V. Smirnova, Redox regulation of cellular functions. Biochemistry. Biokhimiia, 2007. 72(2): p. 132-45.

19. Schieber, M. and N.S. Chandel, ROS function in redox signaling and oxidative stress. Current biology : CB, 2014. 24(10): p. R453-62.

20. D'Autreaux, B. and M.B. Toledano, ROS as signalling molecules: mechanisms that generate specificity in ROS homeostasis. Nature reviews. Molecular cell biology, 2007. 8(10): p. 813-24.

21. van Gisbergen, M.W., et al., How do changes in the mtDNA and mitochondrial dysfunction influence cancer and cancer therapy? Challenges, opportunities and models. Mutat Res Rev Mutat Res, 2015. 764: p. 16-30.

22. Guillaumond, F., et al., Strengthened glycolysis under hypoxia supports tumor symbiosis and hexosamine biosynthesis in pancreatic adenocarcinoma. Proceedings of the National Academy of Sciences of the United States of America, 2013. 110(10): p. 3919-24.

23. Steinbach, J.P., et al., Hypoxia-induced cell death in human malignant glioma cells: energy deprivation promotes decoupling of mitochondrial cytochrome c release from caspase processing and necrotic cell death. Cell death and differentiation, 2003. 10(7): p. 823-32. 
24. Wallace, D.C., Why do we still have a maternally inherited mitochondrial DNA? Insights from evolutionary medicine. Annual review of biochemistry, 2007. 76: p. 781-821.

25. Schon, E.A., S. DiMauro, and M. Hirano, Human mitochondrial DNA: roles of inherited and somatic mutations. Nature reviews. Genetics, 2012. 13(12): p. 878-90.

26. Calabrese, C., et al., Respiratory complex I is essential to induce a Warburg profile in mitochondriadefective tumor cells. Cancer \& metabolism, 2013. 1(1): p. 11.

27. Garcia-Heredia, J.M. and A. Carnero, Decoding Warburg's hypothesis: tumor-related mutations in the mitochondrial respiratory chain. Oncotarget, 2015. 6(39): p. 41582-99.

28. Iommarini, L., et al., Different mtDNA mutations modify tumor progression in dependence of the degree of respiratory complex I impairment. Human molecular genetics, 2014. 23(6): p. 1453-66.

29. Sugioka, K., et al., Mechanism of O2-generation in reduction and oxidation cycle of ubiquinones in a model of mitochondrial electron transport systems. Biochimica et biophysica acta, 1988. 936(3): p. 377-85.

30. Turrens, J.F. and A. Boveris, Generation of superoxide anion by the NADH dehydrogenase of bovine heart mitochondria. The Biochemical journal, 1980. 191(2): p. 421-7.

31. Chen, Q., et al., Production of reactive oxygen species by mitochondria: central role of complex III. The Journal of biological chemistry, 2003. 278(38): p. 36027-31.

32. Muller, F.L., Y. Liu, and H. Van Remmen, Complex III releases superoxide to both sides of the inner mitochondrial membrane. The Journal of biological chemistry, 2004. 279(47): p. 49064-73.

33. Gasparre, G., et al., Relevance of mitochondrial genetics and metabolism in cancer development. Cold Spring Harbor perspectives in biology, 2013. 5(2).

34. Holmstrom, K.M. and T. Finkel, Cellular mechanisms and physiological consequences of redox-dependent signalling. Nature reviews. Molecular cell biology, 2014. 15(6): p. 411-21.

35. Joiner, M. and A.v.d. Kogel, Basic clinical radiobiology, 2009, Hodder Arnold: London. p. 1 online resource (vi, 375 p.).

36. Valko, M., et al., Free radicals, metals and antioxidants in oxidative stress-induced cancer. Chemicobiological interactions, 2006. 160(1): p. 1-40.

37. Porporato, P.E., et al., A mitochondrial switch promotes tumor metastasis. Cell reports, 2014. 8(3): p. 754-66.

38. Lu, J. and A. Holmgren, The thioredoxin antioxidant system. Free radical biology \& medicine, 2014. 66: p. 75-87.

39. Nordberg, J. and E.S. Arner, Reactive oxygen species, antioxidants, and the mammalian thioredoxin system. Free radical biology \& medicine, 2001. 31(11): p. 1287-312.

40. Lu, J., E.H. Chew, and A. Holmgren, Targeting thioredoxin reductase is a basis for cancer therapy by arsenic trioxide. Proceedings of the National Academy of Sciences of the United States of America, 2007. 104(30): p. 12288-93.

41. Du, Y., et al., Glutathione and glutaredoxin act as a backup of human thioredoxin reductase 1 to reduce thioredoxin 1 preventing cell death by aurothioglucose. The Journal of biological chemistry, 2012. 287(45): p. 38210-9.

42. Luo, W., et al., Glutathione s-transferases in pediatric cancer. Frontiers in oncology, 2011. 1: p. 39.

43. Townsend, D.M. and K.D. Tew, The role of glutathione-S-transferase in anti-cancer drug resistance. Oncogene, 2003. 22(47): p. 7369-75.

44. Johansson, K.;Microsomal Glutahione Transferase 1 in anti-cancer drug resistance and protection against oxidative stress'. (Thesis for doctoral degree, Ph.D.), 2010 Karolinska Institutet Stockholm, Sweden. ISBN: 978-91-7409-832-7.

45. Voets, A.M.;New pathophysiological concepts and potential therapeutic targets for oxidative phosphorylation disorders'. (Thesis for doctoral degree, Ph.D.), 2012 Maastricht University Maastricht, The Netherlands. ISBN: 978-90-8891-411-9.

46. Sabharwal, S.S. and P.T. Schumacker, Mitochondrial ROS in cancer: initiators, amplifiers or an Achilles' heel? Nature reviews. Cancer, 2014. 14(11): p. 709-21.

47. Fulda, S., L. Galluzzi, and G. Kroemer, Targeting mitochondria for cancer therapy. Nature reviews. Drug discovery, 2010. 9(6): p. 447-64. 
48. Morgenstern, R., J. Zhang, and K. Johansson, Microsomal glutathione transferase 1: mechanism and functional roles. Drug metabolism reviews, 2011. 43(2): p. 300-6.

49. Siritantikorn, A., et al., Protection of cells from oxidative stress by microsomal glutathione transferase 1. Biochemical and biophysical research communications, 2007. 355(2): p. 592-6.

50. Johansson, K., et al., Multiple roles of microsomal glutathione transferase 1 in cellular protection: a mechanistic study. Free radical biology \& medicine, 2010. 49(11): p. 1638-45.

51. Johansson, K., et al., Characterization of new potential anticancer drugs designed to overcome glutathione transferase mediated resistance. Molecular pharmaceutics, 2011. 8(5): p. 1698-708.

52. Scotlandi, K., et al., Overcoming resistance to conventional drugs in Ewing sarcoma and identification of molecular predictors of outcome. Journal of clinical oncology : official journal of the American Society of Clinical Oncology, 2009. 27(13): p. 2209-16.

53. Tew, K.D. and D.M. Townsend, Redox platforms in cancer drug discovery and development. Current opinion in chemical biology, 2011. 15(1): p. 156-61.

54. Waypa, G.B., et al., Hypoxia triggers subcellular compartmental redox signaling in vascular smooth muscle cells. Circulation research, 2010. 106(3): p. 526-35.

55. Chandel, N.S., et al., Mitochondrial reactive oxygen species trigger hypoxia-induced transcription. Proceedings of the National Academy of Sciences of the United States of America, 1998. 95(20): p. 11715-20.

56. Guzy, R.D., et al., Mitochondrial complex III is required for hypoxia-induced ROS production and cellular oxygen sensing. Cell metabolism, 2005. 1(6): p. 401-8.

57. Paddenberg, R., et al., Essential role of complex /l of the respiratory chain in hypoxia-induced ROS generation in the pulmonary vasculature. American journal of physiology. Lung cellular and molecular physiology, 2003. 284(5): p. L710-9.

58. Sabharwal, S.S., et al., Peroxiredoxin-5 targeted to the mitochondrial intermembrane space attenuates hypoxia-induced reactive oxygen species signalling. The Biochemical journal, 2013. 456(3): p. 337-46.

59. Bell, E.L., et al., The Qo site of the mitochondrial complex III is required for the transduction of hypoxic signaling via reactive oxygen species production. The Journal of cell biology, 2007. 177(6): p. 1029-36.

60. Sullivan, L.B. and N.S. Chandel, Mitochondrial reactive oxygen species and cancer. Cancer \& metabolism, 2014. 2: p. 17.

61. Porporato, P.E. and P. Sonveaux, Paving the way for therapeutic prevention of tumor metastasis with agents targeting mitochondrial superoxide. Molecular \& cellular oncology, 2015. 2(3): p. e968043.

62. Sharma, L.K., et al., Mitochondrial respiratory complex I dysfunction promotes tumorigenesis through ROS alteration and AKT activation. Human molecular genetics, 2011. 20(23): p. 4605-16.

63. Policastro, L.L., et al., The tumor microenvironment: characterization, redox considerations, and novel approaches for reactive oxygen species-targeted gene therapy. Antioxidants \& redox signaling, 2013. 19(8): p. 854-95.

64. Sayin, V.I., et al., Antioxidants accelerate lung cancer progression in mice. Science translational medicine, 2014. 6(221): p. 221ra15.

65. Holohan, C., et al., Cancer drug resistance: an evolving paradigm. Nature reviews. Cancer, 2013. 13(10): p. 714-26.

66. Volm, M. and T. Efferth, Prediction of Cancer Drug Resistance and Implications for Personalized Medicine. Frontiers in oncology, 2015. 5: p. 282.

67. Swietach, P., et al., Importance of intracellular pH in determining the uptake and efficacy of the weakly basic chemotherapeutic drug, doxorubicin. PLoS One, 2012. 7(4): p. e35949.

68. Wojtkowiak, J.W., et al., Drug resistance and cellular adaptation to tumor acidic pH microenvironment. Molecular pharmaceutics, 2011. 8(6): p. 2032-8.

69. Chen, L., et al., Effect of hypoxia-inducible factor-1alpha silencing on the sensitivity of human brain glioma cells to doxorubicin and etoposide. Neurochemical research, 2009. 34(5): p. 984-90.

70. Hao, J., et al., Effects of lentivirus-mediated HIF-1alpha knockdown on hypoxia-related cisplatin resistance and their dependence on p53 status in fibrosarcoma cells. Cancer gene therapy, 2008. 15(7): p. 449-55. 
71. Sasabe, E., et al., The involvement of hypoxia-inducible factor-1alpha in the susceptibility to gamma-rays and chemotherapeutic drugs of oral squamous cell carcinoma cells. International journal of cancer, 2007. 120(2): p. 268-77.

72. Peng, X.H., et al., Cross-talk between epidermal growth factor receptor and hypoxia-inducible factor1alpha signal pathways increases resistance to apoptosis by up-regulating survivin gene expression. The Journal of biological chemistry, 2006. 281(36): p. 25903-14.

73. Chang, Q., et al., Effect of antisense hypoxia-inducible factor 1alpha on progression, metastasis, and chemosensitivity of pancreatic cancer. Pancreas, 2006. 32(3): p. 297-305.

74. Rohwer, N. and T. Cramer, Hypoxia-mediated drug resistance: novel insights on the functional interaction of HIFs and cell death pathways. Drug resistance updates : reviews and commentaries in antimicrobial and anticancer chemotherapy, 2011. 14(3): p. 191-201.

75. Overgaard, J., Hypoxic modification of radiotherapy in squamous cell carcinoma of the head and neck--a systematic review and meta-analysis. Radiotherapy and oncology : journal of the European Society for Therapeutic Radiology and Oncology, 2011. 100(1): p. 22-32.

76. Nordsmark, M., et al., Prognostic value of tumor oxygenation in 397 head and neck tumors after primary radiation therapy. An international multi-center study. Radiotherapy and oncology : journal of the European Society for Therapeutic Radiology and Oncology, 2005. 77(1): p. 18-24.

77. Milosevic, M., et al., Tumor hypoxia predicts biochemical failure following radiotherapy for clinically localized prostate cancer. Clinical cancer research : an official journal of the American Association for Cancer Research, 2012. 18(7): p. 2108-14.

78. Decensi, A., et al., Metformin and cancer risk in diabetic patients: a systematic review and meta-analysis. Cancer prevention research, 2010. 3(11): p. 1451-61.

79. Wheaton, W.W., et al., Metformin inhibits mitochondrial complex I of cancer cells to reduce tumorigenesis. eLife, 2014. 3: p. e02242.

80. Zhou, G., et al., Role of AMP-activated protein kinase in mechanism of metformin action. The Journal of clinical investigation, 2001. 108(8): p. 1167-74.

81. Madiraju, A.K., et al., Metformin suppresses gluconeogenesis by inhibiting mitochondrial glycerophosphate dehydrogenase. Nature, 2014. 510(7506): p. 542-6.

82. Miller, R.A., et al., Biguanides suppress hepatic glucagon signalling by decreasing production of cyclic AMP. Nature, 2013. 494(7436): p. 256-60.

83. Zannella, V.E., et al., Reprogramming metabolism with metformin improves tumor oxygenation and radiotherapy response. Clinical cancer research : an official journal of the American Association for Cancer Research, 2013. 19(24): p. 6741-50.

84. Storozhuk, Y., et al., Metformin inhibits growth and enhances radiation response of non-small cell lung cancer (NSCLC) through ATM and AMPK. British journal of cancer, 2013. 108(10): p. 2021-32.

85. Skinner, H.D., et al., TP53 disruptive mutations lead to head and neck cancer treatment failure through inhibition of radiation-induced senescence. Clinical cancer research : an official journal of the American Association for Cancer Research, 2012. 18(1): p. 290-300.

86. Skinner, H.D., et al., Metformin use and improved response to therapy in rectal cancer. Cancer medicine, 2013. 2(1): p. 99-107.

87. Skinner, H.D., et al., Metformin use and improved response to therapy in esophageal adenocarcinoma. Acta oncologica, 2013. 52(5): p. 1002-9.

88. Ferro, A., et al., Evaluation of Diabetic Patients with Breast Cancer Treated with Metformin during Adjuvant Radiotherapy. International journal of breast cancer, 2013. 2013: p. 659723.

89. Cheng, G., et al., Mitochondria-Targeted Analogues of Metformin Exhibit Enhanced Antiproliferative and Radiosensitizing Effects in Pancreatic Cancer Cells. Cancer research, 2016. 76(13): p. 3904-15.

90. Appleyard, M.V., et al., Phenformin as prophylaxis and therapy in breast cancer xenografts. Br J Cancer, 2012. 106(6): p. 1117-22.

91. Yuan, P., et al., Phenformin enhances the therapeutic benefit of BRAF(V600E) inhibition in melanoma. Proc Natl Acad Sci U S A, 2013. 110(45): p. 18226-31. 


\section{Chapter 1}

92. Navarro, P., et al., Targeting Tumor Mitochondrial Metabolism Overcomes Resistance to Antiangiogenics. Cell Rep, 2016. 15(12): p. 2705-18.

93. Sonveaux, P., et al., Nitric oxide delivery to cancer: why and how? European journal of cancer, 2009. 45(8): p. 1352-69.

94. Yasuda, H., Solid tumor physiology and hypoxia-induced chemo/radio-resistance: novel strategy for cancer therapy: nitric oxide donor as a therapeutic enhancer. Nitric oxide : biology and chemistry / official journal of the Nitric Oxide Society, 2008. 19(2): p. 205-16.

95. Redza-Dutordoir, M. and D.A. Averill-Bates, Activation of apoptosis signalling pathways by reactive oxygen species. Biochimica et biophysica acta, 2016. 1863(12): p. 2977-2992.

96. Lee, S., et al., An effective strategy for increasing the radiosensitivity of Human lung Cancer cells by blocking Nrf2-dependent antioxidant responses. Free Radic Biol Med, 2012. 53(4): p. 807-16.

97. Yamamori, T., et al., lonizing radiation induces mitochondrial reactive oxygen species production accompanied by upregulation of mitochondrial electron transport chain function and mitochondrial content under control of the cell cycle checkpoint. Free Radic Biol Med, 2012. 53(2): p. 260-70.

98. Koritzinsky, M., Metformin: A Novel Biological Modifier of Tumor Response to Radiation Therapy. International journal of radiation oncology, biology, physics, 2015. 93(2): p. 454-64. 


\section{Chapter}

\section{How do changes in the mtDNA and mitochondrial dysfunction influence cancer and cancer therapy? Challenges, opportunities and models}

Published in: Mutation research. Reviews in mutation research, 2015. 764: p.16-30. doi: 10.1016/j.mrrev.2015.01.001

Marike W. van Gisbergen, An M. Voets, Maud H.W. Starmans, Irenaeus F.M. de Coo, Rana Yadak, Roland F. Hoffmann, Paul C. Boutros, Hubertus J.M. Smeets, Ludwig Dubois, Philippe Lambin 


\section{Abstract}

Several mutations in nuclear genes encoding for mitochondrial components have been associated with an increased cancer risk or are even causative, e.g. succinate dehydrogenase (SDHB, SDHC and SDHD genes) and iso-citrate dehydrogenase (IDH1 and IDH2 genes). Recently, studies have suggested an eminent role for mitochondrial DNA (mtDNA) mutations in the development of a wide variety of cancers. Various studies associated mtDNA abnormalities, including mutations, deletions, inversions and copy number alterations, with mitochondrial dysfunction. This might, explain the hampered cellular bioenergetics in many cancer cell types. Germline (e.g. m.10398A>G; m.6253T>C) and somatic mtDNA mutations as well as differences in mtDNA copy number seem to be associated with cancer risk. It seems that mtDNA can contribute as driver or as complementary gene mutation according to the multiple-hit model. This can enhance the mutagenic/clonogenic potential of the cell as observed for m.8993T>G or influences the metastatic potential in later stages of cancer progression. Alternatively, other mtDNA variations will be innocent passenger mutations in a tumor and therefore do not contribute to the tumorigenic or metastatic potential. In this review, we discuss how reported mtDNA variations interfere with cancer treatment and what implications this has on current successful pharmaceutical interventions. Mutations in MT-ND4 and mtDNA depletion have been reported to be involved in cisplatin resistance. Pharmaceutical impairment of OXPHOS by metformin can increase the efficiency of radiotherapy. To study mitochondrial dysfunction in cancer, different cellular models (like $\rho^{0}$ cells or cybrids), in vivo murine models (xenografts and specific mtDNA mouse models in combination with a spontaneous cancer mouse model) and small animal models (e.g. Danio rerio) could be potentially interesting to use. For future research, we foresee that unraveling mtDNA variations can contribute to personalized therapy for specific cancer types and improve the outcome of the disease. 


\section{Introduction: the cancer's cell energy supply}

In normal physiology, mitochondria are very important in the cell as they produce most of the adenosine triphosphate (ATP) via the oxidative phosphorylation system (OXPHOS), which is a necessary energy supply for cellular processes. The OXPHOS system consists of five protein complexes (complex I-V). All complexes are partly encoded by the mitochondrial DNA (mtDNA), except for complex II, which is completely nuclear encoded [1]. The OXPHOS system also consists of electron carriers that are situated in the inner mitochondrial membrane (Fig. 1B) [2]. Approximately $0.15-2 \%$ of the electrons that enter the electron transport chain (ETC) [3] can escape the OXPHOS cycle, resulting in the formation of superoxide. Increased exposure to this OXPHOSrelated superoxide may not only affect the nearby located mtDNA but also the nuclear DNA (nDNA), proteins and lipids, resulting in impaired proteins and/or enhanced reactive oxygen species (ROS) production [4]. mtDNA mutations can lead to a decreased efficiency of the ETC and can cause more ROS production [5][6]. It has been shown both in vitro and in vivo that the OXPHOS system and OXPHOS-related superoxide can have a major influence on tumor progression [7]. As the electron transport chain is the most important site of ROS generation, mutations in mIDNA can cause more ROS production [8]. Evidence suggests when mitochondrial integrity is compromised by excessive ROS formation or mtDNA instability, cancer progression is enhanced. Increased ROS levels and oxidative damage are observed in fibroblasts of patients with mtDNA mutations [6]. To protect against ROS, cells have an antioxidant network from which important antioxidant enzyme groups are catalases, glutathione peroxidases, thioredoxins, peroxiredoxins, glutathione transferases and superoxide dismutases (SODs). SODs are capable of catalyzing the dismutation of superoxide into hydrogen peroxide and oxygen [9]. One of these SOD family members, manganese superoxide dismutase (MnSOD) (Fig. 1B), is located in the matrix of mitochondria [10]. Many types of human cancer cells have reduced MnSOD activity compared to their originated cells [11]. Increased MnSOD expression was able to suppress tumor cell growth and tumor formation in MCF7, A172R, U118, SCC25 and DU145 cells [12]. It has been suggested that MnSOD is a tumor suppressor gene [13] and that mutations found in its promoter region could explain the reduced MnSOD activity [14]. Additionally, also antioxidants are involved in this network. The hydrophilic non-protein thiol, glutathione (GSH), is an important ROS scavenger. In a cell, GSH mainly exits in its reduced form, but is able to oxidize into glutathione disulfide (GSSG), with a ratio between 30:1 and 100:1. However, this ratio can decrease upon oxidative stress [15]. Additionally, GSSG can be converted back into GSH driven by glutathione reductase, the catalyzer for this reaction [16]. An inadequate antioxidant response, where antioxidants are not able to maintain 'normal' physiological ROS levels, will lead to a vicious circle of ROS production and create cellular damage $[6,17]$ 
Warburg observed in 1927 that cancer cells rely less on the OXPHOS system, but rather on glycolysis for ATP production and therefore produce high amounts of lactate, even in the presence of oxygen. This phenomenon is known as the Warburg effect, or aerobic glycolysis [18]. Many cancer types have a tendency to be highly glycolytic and mitochondria in cancers cells also show altered cristae, membrane composition and membrane potential [4][19], resulting in an aberrant mitochondrial function influencing ROS production and apoptosis [4, 20]. ROS production can be increased due to aberrant mitochondrial function causing cells to be more prone to apoptosis by activating the mitochondrial permeability transition pore [21-23]. However, increased apoptosis could also be independent of ROS as observed in for instance the mutator mouse model [24]. In cancer, inhibition of factors initiating apoptosis could drive cells toward an actively proliferating state with ROS escalation and DNA damage. The paradox between ROS and apoptosis, in aging or cancer has been extensively reviewed by Wallace [22, 25]. Mitochondrial dysfunction can be observed in tumors, particularly in those that are aggressive and growing rapidly $[26,27]$. Simonnet et al. compared mitochondrial enzymes and DNA contents in renal cell carcinoma (RCC) to normal kidney tissue. Mitochondrial impairment was increased from the less aggressive to the most aggressive RCCS and correlated with a considerably decreased content of OXPHOS complexes [27]. To maintain optimal ATP levels, some tumor cells are able to stimulate the glycolysis pathway upon pharmacological inhibition of the OXPHOS system. In contrary, after inhibition of glycolysis, the OXPHOS system cannot compensate for the loss of ATP production in these same cells $[28,29]$. This is, however, not universal as other tumor cell lines are still capable of maintaining high ATP contents when they are deprived of glucose $[30,31]$. The extent of changes in mitochondrial respiratory capacity seems to depend on the tumor type and its microenvironmental characteristics, such as hypoxia and hormones [32-34]. Adaptation of these processes might affect the tumor responsiveness to therapies, which points to a potential therapeutic target for new cancer therapies. 
A

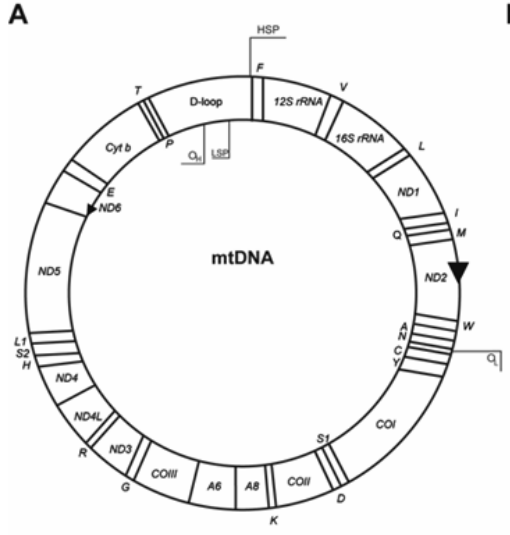

B

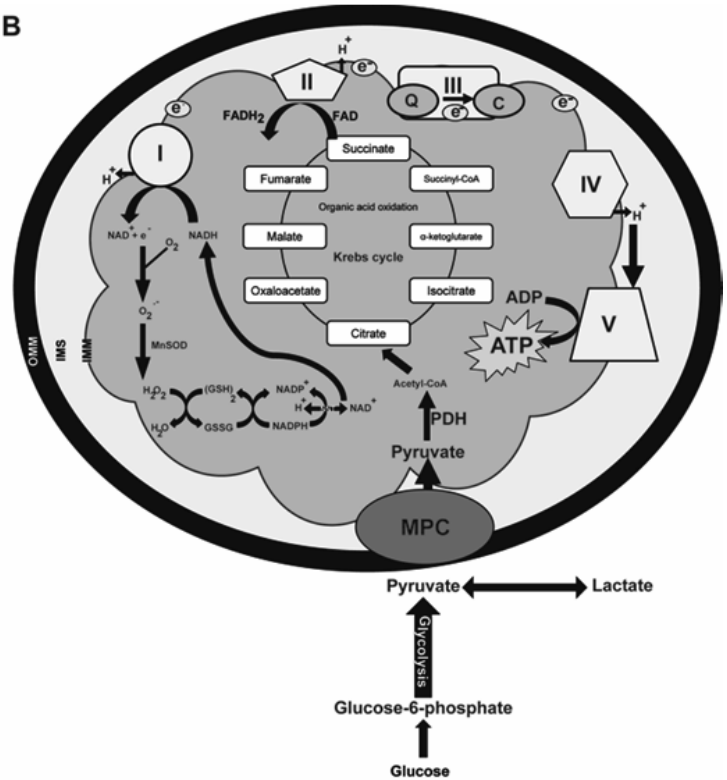

Figure 1. Global representation of the OXPHOS system and the link to glycolysis in a cell. A.

Overview of the mitochondrial DNA. The genes coding for subunits of OXPHOS complex I are ND1-ND6 and ND4L. Genes for cytochrome c oxidase (complex IV) are COI-COIII. The gene encoding for Cytochrome B of complex III abbreviated with Cyt B. Additionally the subunits of Complex V (ATP synthase) are ATPase 6 and 8 abbreviated with $A 6$ and $A 8$ respectively. The 22 tRNAs are indicated with a single letter. The two ribosomal RNAs encoded by the mtDNA are $12 \mathrm{~S}$ and $16 \mathrm{~S}$. The displacement loop is represented as D-loop and contains sequences for initiation for replication and transcription including the origin of heavy-strand replication $(\mathrm{OH})$. The light strand replication origin is indicated by OL. The position of the Light strand promoter is shown as LSP and the position of the heavy-strand promotor as HSP. B. Overview of OXPHOS system. When glucose enters the cell it is converted to glucose-6-phosphate, after which it undergoes glycolysis. The final step of glycolysis is the formation of pyruvate and reduced nicotinamide adenine dinucleotide (NADH). Pyruvate can be either transformed into lactate and nicotinamide adenine dinucleotide (NAD) by lactate dehydrogenase (LDH) or can be transported immediately into the mitochondria. This transport is mediated by a mitochondrial pyruvate carrier (MPC) which transports pyruvate across the outer mitochondrial membrane (OMM), the intermembrane space (IMS) and the inner mitochondrial membrane (IMM) into the mitochondrial matrix, where pyruvate dehydrogenase (PDH) catalysis it is converted into acetyl coenzyme A (acetyl CoA). At the same time, the MPC transports hydroxide out of the mitochondria. Acetyl CoA is able to enter the Krebs cycle, where organic acid oxidation takes place and results in succinate production as part of the Krebs cycle and NADH. Electrons from NADH can enter the electron transport chain (ETC), formed by complex I, complex II, complex III and complex IV (I-IV). $\mathrm{Cl}$ is also known as NADH dehydrogenase / NADH ubiquinone oxidoreductase, the largest of the oxidative phosphorylation (OXPHOS) complexes, and is one of the starting points of enrolling the electrons into the ETC [5]. The ETC transports electrons through the IMM, towards coenzyme $\mathrm{Q}(\mathrm{Q})$, after which they are transferred to complex III (III). The electrons can also skip $\mathrm{Cl}$ and be transported via flavin containing enzyme complexes directly towards $Q$ [2]. Another route for electron donation to the ETC is via complex II, succinate dehydrogenase/ succinate ubiquinone oxidoreductase (II), where succinate is reduced and electrons are transferred towards coenzyme $Q$ and complex III (III). Via complex III and cytochrome $c(\mathrm{C})$, electrons are moved towards CIV. During electron transport, a proton gradient is formed over the IMM by $\mathrm{Cl}, \mathrm{CIII}$ and CIV, which drives protons into the mitochondrial matrix via, CV, ATP synthase, (V) with concomitant production of ATP [2, 35]. All complexes combined are called OXPHOS. 
In total, OXPHOS can produce up to $36 \mathrm{~mol}$ of ATP per mol of glucose[36]. Additionally, reactive oxygen species can be formed which can be transferred into $\mathrm{H}_{2} \mathrm{O}_{2}$ by manganese superoxide dismutases (MnSOD). $\mathrm{H}_{2} \mathrm{O}_{2}$ and glutathione can be transformed into $\mathrm{H}_{2} \mathrm{O}$ and oxidized under the influence of glutathione peroxidase (GPX). Via glutathione reductase (GR) and nicotinamide (NNT) eventually $N A D^{+}$can be formed again.

\section{Mutations in nuclear-encoded mitochondrial genes can cause cancer}

Next to the conventional cancer causing mutations (e.g. TP53 encoding for p53 which is involved in proliferation, apoptosis and DNA repair [37-39]), some cancers (e.g. paraganglioma) are found to be associated with a mutation in one of the nuclear mitochondrial genes encoding for OXPHOS subunits or parts of the Krebs cycle. Patients suffering from this disease have a dysfunctional succinate dehydrogenase $(S D H)$, better known as complex II, which is composed of four subunits. Whereas mutations in subunit A are mostly responsible for Leigh's disease (a rare neurometabolic disorder), mutations in subunits B, C or D mainly cause paraganglioma [40-43]. In a xenograft study studying a SDHC missense mutation it was found that mice formed benign tumors comparable with paragangliomas [43]. In 2 other studies investigating families with paragangliomas carrying mutations in SDHD [41] or SDHC [42] a causative role was found for these mutations and a tumor suppressor function for the genes was suggested [41, 42].

Nevertheless, a family with mutated SDHA was recently reported to have paraganglioma as well [44]. A proposed mechanism in patients could be that mitochondrial ROS increases and more oxidative damage occurs. This could result in oncogenic transformation as occurs for paragangliomas [43].

Additionally, mutations in succinate dehydrogenase have been found in breast cancer, gastrointestinal stromal cancer and renal carcinomas [45-47]. For other cancers like uterine leiomyomas and RCCs $[48,49]$, associations with mutations were found in other nuclear-encoded mitochondrial genes such as fumarate hydratase. Heterozygous mutations have been reported in the gene encoding for fumarate hydratase, which catalyzes the reversible hydration of fumarate to malate, a Krebs cycle substrate (see Fig. 1B) [50]. Similarly, for iso-citrate dehydrogenase, catalyzing the conversion of isocitrate into 2-glutarate in the Krebs cycle, mutations in the cytoplasmic (IDH1) and the mitochondrial (IDH2) isoforms are reported in a wide variety of human cancer types such as: acute myeloid leukemia [51, 52], angioimmunoblastic T-cell lymphomas [53], cartilaginous tumors [54], colorectal cancer [55], glioblastomas [56], glioma [57] and prostate cancer [58]. For a more extended review see Gaude and Frezza [59].

Although, evidence for a causative role of the latter mentioned mutations in oncogenesis is mostly indirect, the consequent changes in metabolite levels such as succinate have been shown to influence hypoxia inducible factor (HIF) stabilization [60]. 


\section{Mitochondrial DNA mutations and cancer}

Over recent years, accumulating evidence suggests that mtDNA mutations may also contribute to a cell's potential to become a cancer cell [61-63], ultimately leading to tissue invasion and metastasis. mtDNA variations, such as deletions, point mutations and copy number differences, are associated with a wide variety of cancer types [64-74].

\section{Genetic insight into mtDNA}

mtDNA characteristics

The mtDNA is a $16.5 \mathrm{~kb}$ double-stranded, circular-shaped DNA molecule that is distinct from nDNA in several ways: (1) mtDNA is only maternally inherited; (2) mammalian cells can contain thousands of mtDNA molecules, but they nevertheless represent only a minor percentage of the total DNA [75]. As mtDNA is polyploid, a mitochondrion contains five to ten copies of mtDNA, which can differ in composition from each other. Homoplasmy indicates that the mtDNA copies in a cell are all identical, while a mixture of wild-type and variant copies is referred to as heteroplasmy [76]; (3) mtDNA is almost completely comprised of coding sequence. It codes for 13 OXPHOS subunits, 22 tRNAs and 2 rRNAs; less than 10 percent of the entire mtDNA is non-coding [1] (Fig. 1A). This non-coding region is mainly located in the displacement (D)-loop, a $1.1 \mathrm{~kb}$ region involved in mtDNA replication and transcription. In addition, mtDNA encoded genes do not contain introns and have none or only a few non-coding bases between them. The coding sequences of some of the encoded genes are even overlapping, i.e. they share several mtDNA bases [1]. Consequently, changes in the mtDNA are more likely to have functional consequences than nDNA variations [70, 77, 78]; (4) in the mitochondrial genome, transcription starts from one of the two mitochondrial promoters and can take place on both DNA strands. Subsequently, transcription produces a polycistronic precursor RNA that results in individual tRNA, rRNA and mRNA molecules after processing; (5) mtDNA replication occurs independent of the cell cycle which is therefore referred to as relaxed replication. This means that the number of mtDNA molecules replicated per cell cycle is only restricted by the levels of available replication machinery factors and nucleotides [79, 80]; (6) mtDNA has a higher mutation frequency compared to the nDNA in mammals $[81,82]$. Possible explanations are: mtDNA is located close to the ROS producing OXPHOS and mtDNA lacks protective histones [83, 84]. However, there is a debate about the protective role of histones, as there is also evidence showing that the electrons can transfer easily from histones to DNA leading to damage [85] and under some conditions (exposure to $\mathrm{Cu}(\mathrm{II}) / \mathrm{H}_{2} \mathrm{O}_{2}$ ) histones can even enhance DNA damage [86]. It is also suggested that DNA-binding proteins of mitochondrial nucleoids can be as equally protective as histones for mtDNA under $\mathrm{H}_{2} \mathrm{O}_{2}$ or X-ray exposure $[87,88]$. 
Mitochondrial DNA maintenance

The replication of mtDNA is maintained via DNA polymerase $\gamma$ (POLG1 and POLG2 genes) [89], mitochondrial transcription factor A (TFAM) [90], Twinkle (C10orf2) [44], mitochondrial 12S rRNA dimethylase 1 and mitochondrial 12S rRNA dimethylase 2 (TFB1M and TFB2M, synonyms: mtTFB or Mtf1) [50], mitochondrial single stranded DNA binding protein (mtSSB) [91] and mitochondrial RNA polymerase gene (POLRMT) [74]. Both polymerase $\gamma$ and TFAM are essential for maintaining mtDNA copy number and integrity [92-96]. More frequent point mutations and deletions were observed when introducing mutations in the exonuclease domain of POLG resulting in misincorporation of nucleotides [97, 98]. Furthermore, compared to nDNA, mtDNA damage persists longer [84].

In murine models having a complete knockout for TFAM, loss of mtDNA and severe OXPHOS defects are observed leading to embryonic death [96]. Inducible knock-down leads to cell death of the targeted cell type [99, 100]. In 2011, Balliet and colleagues showed that fibroblasts lacking TFAM exhibited mitochondrial dysfunction and increased oxidative stress due to the loss of certain OXPHOS components and overproduction of hydrogen peroxide and lactate [101].

mtDNA dynamics

Mitochondria are able to change their number and shape in different cell types under varying physiological conditions $[102,103]$. One possible way for mitochondria to maintain their integrity and thus to ensure a healthy population is by exchanging mtDNA through constant fission and fusion processes. It can guard a cell by allowing the mitochondria to fuse or divide and thereby protect the cell from detrimental effects of (accumulating) mtDNA mutations [104].

Mitophagy, a degradation process of mitochondria through autophagy, controls the number of mitochondria in the cell and initiates the removal of dysfunctional and damaged mitochondria [105]. Previous studies had already shown that defective mitochondria displayed increased mitophagy $[106,107]$. So when a mutation leads to a reduced mitochondrial membrane potential (depolarization of mitochondria) the mutation causes a phenotypical change and mitochondria loose therefore their ability to function normal. Consequently, the mitochondria are not able to re-fuse with the mitochondrial network after fission and are recycled [108].

When a somatic mtDNA mutation first occurs, it will be in one mtDNA molecule. The process of random genetic drift, i.e. the change in the frequency of a variant in a population due to random sampling, will at first determine the persistence and expansion or loss of this mutation since it will not have significant functional effects and thus selection advantage as a single copy. These mutations are not targeted by mitophagy and via fusion dominant mutations can become homoplasmic [70]. It is 
known from haplogroup studies that mtDNA variation can have an effect on how efficient electron transport and ATP production are coupled in the mitochondria [109]. In that sense, varying percentages of mutant mtDNA can lead to bioenergetic defects ranging from mild mitochondrial dysfunction to severe metabolic distress and cell death [110] or they can lead to more optimal functioning of the OXPHOS process. In combination with the proposed model that cancer metabolism switches from mainly glycolysis to preferentially OXPHOS and back during the cancer process [111], this suggests that mutations reaching certain heteroplasmy levels by random genetic drift resulting in a detrimental (stimulating glycolysis) or beneficial effect on OXPHOS will be selected and clonogenic expanded in these respective metabolism waves.

\section{Germline mtDNA mutations associated with cancer}

Different studies have explored the association between germline mtDNA mutations and cancer. There are indications that different germline mutations could actually contribute to the development of a certain cancer type in a specific population.

Different human populations can be distinguished by different human mtDNA haplogroups which are defined by unique sets of mtDNA polymorphisms, reflecting mutations accumulated by a discrete maternal lineage [112]. The haplogroups are associated with region-specific mtDNA sequence variation as a result of genetic drift and/or adaptive selection for an environment-favored mitochondrial function [109, 113]. Tanaka et al. classified 30 different haplogroups in a retrospective study and found that the risk in the population M7b2 haplogroup was related to increased risk for hematopoietic cancer [114]. Furthermore, Booker et al. showed that haplogroup U was related to a 2.5-fold increased risk for developing renal cancer in Caucasian American men [115]. They also reported a two-fold increased risk for development of prostate cancer, a finding which was supported by Canter et al. [115, 116].

Bai et al. identified two different haplogroups that influence breast cancer risk in a Caucasian population: haplogroup $\mathrm{K}$ indicates an increased risk whereas haplogroup $\mathrm{U}$ is a protective haplogroup [117]. Additionally, they identified four different single nucleotide polymorphisms (SNPs) that influence cancer risks in certain populations.

The m.10398A is especially of interest as it also defines the European haplogroup N [118]. The revised Cambridge Reference Sequence (rCRS) [119] has an A as reference nucleotide. In the paper of van Oven et al. a different sequence was published based on a phylogenetic approach. Here it was shown that the original wild-type nucleotide on this position should be $a, G$ and this site is prone for mutations as the $G>A$ polymorphism occurs in different haplogroups [118]. Annotations in this paper are based on the rCRS nomenclature and HGVS guidelines. 
The m.10398A $>\mathrm{G}$ and $\mathrm{m} .16519 \mathrm{~T}>\mathrm{C}$ variants were found to be related to an increased risk of breast cancer. Two other SNPs, m.13708G $>$ A and m.3197T >C, were identified as protective variations [117]. Interestingly, in a population of African-American women, the m.10398A variant led to an increased risk of invasive breast cancer [120]. In an Indian population with haplogroup N, an association was found between m.10398A and breast and esophageal cancer [121]. However, in another study by Francis et al. within an Indian population no correlation was found between the m.10398A polymorphism and increased breast cancer risk [122]. In a study performed by Mosquera-Miguel et al. also no association was found for this variation in a Spanish population [123]. Recently, Salas et al. reanalyzed all studies and no correlation could be found regarding the m.10398A>G SNP [124].

For prostate cancer, Petros et al. have demonstrated an association for four different germline mutations in cytochrome oxidase subunit I (m.6253T>C, m.6340C $>$ T, m.6261G $>A$, and m.6663A>G) in a cohort study. Additionally, in a proof of principle study they showed for the germline mutation m.8993T>G in ATP6 a 7-fold enhanced tumor volume for mutants compared to wild-type tumors. This indicates that mtDNA germline variations could play a role in tumor growth for prostate cancer [68].

For pancreatic cancer Wang et al. investigated if pancreatic cancer risk increase was associated with mtDNA SNPs however no correlation could be observed [125]. Navaglia et al. showed by sequencing the D-loop region that germline m.16519T worsened the prognosis in pancreatic cancer patients. However, somatic D-loop mutations seemed not to be involved [126]. Also, Halfdanarson et al. investigated if survival outcome in pancreatic cancer could be predicted based on mtDNA variations by using a SNP approach; but they did not find a relationship between certain SNPS and pancreatic survival [127]. However, Lam et al. found using genome wide sequencing an association with pancreatic cancer and the variant $\mathrm{m} .5460 \mathrm{G}>\mathrm{A}$ encoding for a non-synonymous p.A331T substitution in the ND2 gene. In the same study it was found that haplogroup $\mathrm{K}$ was correlated with a reduced risk compared to haplogroup $\mathrm{H}$, however this could be a false positive finding, since these results were inconsistent with previous data and the amount of haplogroup $\mathrm{K}$ participants was very low. Additionally, in haplogroup $\mathrm{N}$ the amount of rare singleton (variants unique to a single participant) variations in HV2 and 12S RNA regions was increased compared to controls. The same was found for singletons ND4 and ND5 among patients with haplogroup L [67]. These findings indicate that mtDNA variations might contribute to pancreatic cancer risk, however this needs to be further investigated.

The mentioned studies above took different approaches to identify mtDNA variations. Multiple studies only looked at certain SNPs [68, 117, 120, 122, 123, 125-127], and others performed complete mtDNA sequencing to pick up variants [67], which give more inside in the different types of variations. It should be taken into account that also 
individual non-synonymous polymorphisms are able to influence mitochondrial function and that restricting analysis to haplogroup variation does not cover the load of mtDNA variation. Epistatic interactions (interactions between 2 or more variations) between the mtDNA and the nDNA are worth investigating in relation to cancer predisposition or development. There seems to be some evidence that germline polymorphisms and haplogroups are related to different cancer types. Note that studies finding germline mtDNA variations often face high frequencies of functional polymorphisms and high mutation rates can lead to the rise of the same mutation in different populations and therefore should be adjusted for population substructures as it is done in similar to Genome Wide Association studies. Otherwise, the SNPs can just mark different ancestral populations and not causal mechanisms [128].

Identifying germline mutations related to a specific cancer type in a defined population can be very difficult, as the same mutation can lead to a different risk in another population. From this perspective, somatic mtDNA mutations have an advantage as they are only carried in the tumor and not the patients' normal tissue [70].

\section{Somatic mtDNA mutations related to cancer}

In 1998, Polyak et al. demonstrated that somatic variations in the mtDNA were present in the primary tumors of colorectal cancer patients [68]. In the following years, numerous somatic mtDNA mutations were reported in a wide variety of tumors (e.g. colorectal, breast, bladder, esophageal, head and neck, ovarian, renal, leukemia, lung and thyroid cancer) [69-71, 129-133]. Furthermore, somatic mutations have been shown to influence cancer progression and metastasis [68, 73, 134, 135]. Data analysis by Larman et al. showed that, across 5 different cancer types, displayed somatic mtDNA mutations ranging, from $13 \%$ in glioblastoma to $63 \%$ frequency in rectal adenocarcinomas [135]. Some data suggests that the effect of the somatic mtDNA mutations and the degree or nature of the tumorigenesis effect depend on the functional and threshold effect of the mutation [136-138]. For instance, the m.3460G>A/MT-ND1 mutation (decrease in complex I activity) result in different tumorigenic potential as determined by colony forming efficiency and tumor growth of osteosarcoma cybrids (cytoplasmic hybrids, Fig. 2) [137] compared to the m.3571insC/MT-ND1 and the m.3243A>G/MT-TL1 mutation (severe structural and functional complex I alteration). More severe alterations in complex I (m.3571insC/MTND1 and the m.3243A>G/MT-TL1 mutation) resulted in a reduced tumorigenic potential both in vitro and in vivo, compared with cells displaying milder complex I dysfunction (m.3460G>A/MT-ND1 mutation) [137]. 


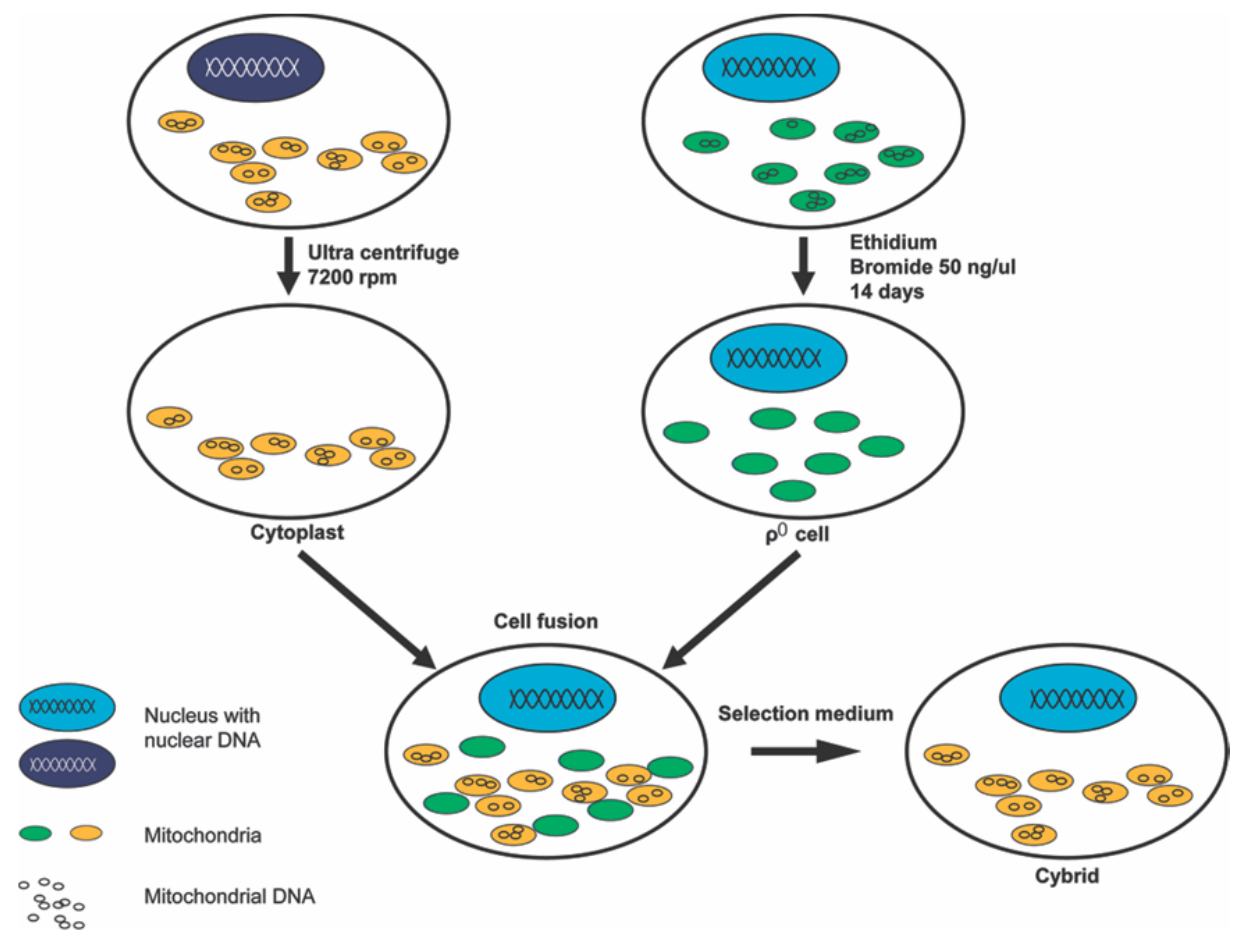

Figure 2. Cybrid model for investigating the influence of mtDNA on cancer.

Cybrids are produced by combining nucleated cells without mtDNA ( $\rho^{0}$ cells) with cytoplasm from nonnucleated cells (cytoplasts). A cytoplast is made by removing the nucleus through ultracentrifugation (7200 RPM), leaving a cell that only contains mitochondria with mtDNA. Nucleated cells without mtDNA ( $\rho^{0}$ cells) are formed by the depletion of the mtDNA by exposing the cells to ethidium bromide (Etbr). As these cells contain nuclear DNA, they define the nuclear background of the cybrid. Fusion of $\rho^{0}$ cells with the cytoplasts leads to the formation of cybrids.

\section{Changes in mtDNA copy number and its association with cancer}

The number of mtDNA molecules could be another factor of consideration since this can affect proper mitochondrial function too [139]. Other reviews describe this subject in more depth or have been exclusively dedicated to this subject (see [140]). Here we give a short summary on this subject; however, this is not a complete list regarding observed changes of mtDNA copy number in cancer. The association between copy number variation and cancer is still subject of debate. Different trends have been reported in multiple tumor types. Both an increase and a decrease in mtDNA copy number have been reported to be associated with an increased risk for tumorigenesis. In genomic DNA extracted from blood an elevated copy number (compared to matched control subjects) has been observed in of patients with various cancers e.g. breast cancer [141], RCCs [142], non-small cell lung cancer [65]. Additionally, there are also studies investigating mtDNA copy number in tumor tissues. In tumor tissues compared 
to matched control subjects an elevated copy number was found for endometrial cancer [143], glioblastoma [144], head and neck cancer [145] and ovarian cancer [146]. Also in tumor tissue compared with paired non-tumor tissue from the same patient an elevated copy number have been found for colorectal carcinoma [147], esophageal squamous cell carcinoma [148], metastatic fibrolamellar carcinomas [149], prostate cancer [150] and thyroid carcinoma [151].

Increased mtDNA copy numbers in tumors could possibly be explained by an increase of oxidative stress [152]. However, the precise mechanisms have not been fully understood. Potentially mtDNA replication could be increased to compensate for metabolic effects caused by mtDNA variations or oxidative stress [140].

In contrast, decreased mtDNA copy numbers have been reported in patients compared to matched tissue subjects for astrocytoma [153] and Ewings sarcoma [154], and in tumor versus non-tumor tissue samples from the same patient for breast cancer [151], lung cancer [155], primary fibrolamellar carcinoma [149], gastric cancer [156], hepatocellular carcinoma [149] and RCC [157]. A twin study found that a reduced mtDNA copy number was related to an increased risk for the development of RCC [158].

Furthermore, there are indications that the depletion of mtDNA in cancer cells can be responsible for disease progression [159]. Lowered mtDNA is correlated with metastasis in patients suffering from Ewing's sarcomas [154]. In addition, shorter survival time was related to low mtDNA copy numbers in patients suffering from astrocytoma [153]. Again, a reverse observation was made for head and neck tumors where increased mtDNA copy number was found to be associated with a decreased survival [160].

Low mtDNA content can be caused by p53 or POLG1 mutations, resulting in tumor initiation or progression [159, 161-163]. The POLG1 gene was found to be mutated in $63 \%$ of breast tumors, and thought to be responsible for a depletion of mtDNA in these tumors [162]. TFAM mutations associated with a lowered TFAM protein and a decreased mitochondrial copy number were found in colorectal cancers with microsatellite instability while no TFAM mutations were observed in colorectal cancers with microsatellite stability. Additionally, TFAM mutated cells (TFAM frame-shift mutation) were able to grow larger tumors in vivo [95] and lowered mtDNA copy number and more oxidative mtDNA damage was observed in $\operatorname{TFAM}^{+/-}$mice $[96,164]$. When this model was crossbred with the adenomatous polyposis coli multiple intestinal neoplasia $\left(\mathrm{APC}^{-/+}\right)$mouse cancer model, mtDNA instability enhanced tumorigenesis [164]. These findings suggest that both polymerase $\gamma$ and TFAM might play an important role in tumorigenesis. Although, it is not investigated if POLG1 and TFAM mutations influence oncogenic transformation as such, a promotion of oncogenic potential after primary transformation has been shown especially for TFAM [95].

There have been several reports of higher cancer incidence with both increased and decreased copy numbers in the same tumor type. An example is RCC, for which both 
increased [142] and decreased copy numbers has been described [157][158]. Furthermore, there seems to be no specificity for tumor type or primary site. The exact molecular and metabolic differences between the cancer types that relate to these copy number differences should be examined in more detail for a plausible rationale to be found.

Previously, we discussed several types of nuclear encoded mitochondrial and mtDNA mutations exhibiting mitochondrial dysfunction and how they relate to cancer and cancer therapy (see Fig. 3). (1) Certain mutations display a causative effect for nuclear encoded mitochondrial and mtDNA genes in cancer. Some of the mutations in nuclear encoded mitochondrial genes have been reported to behave like tumor suppressor genes (like SDH or MnSOD) and loss of function or lowered expression increases tumor formation. Additionally, it has also been reported that mtDNA depletion can result in a tumorigenic phenotype in vitro as well as in vivo [53]. (2) The multiple-hit model could be an explanation for the observed alterations of mtDNA variations observed in cancer as most scientific findings presented only an association of mtDNA mutations in cancer. These mutations are not directly involved in generating the neoplastic phenotype but can be responsible for tumor progression or play an important role in the metastatic potential of the tumors. Additionally, such mutations can also influence the outcome of disease by promoting cancer treatment insensitivity. However, there is also a second mechanism for the multiple-hit model possible. In this mechanism some driver mutations, without direct selective value, can be randomly fixed via random-drift. In this manner, they only express their clonogenic or metastatic potential at a later stage and only in combination with other driver mutations. (3) Another option could be that neutral mtDNA mutations occur random and are not related to clonogenic potential or metastatic potential and are subject to genetic drift (= can disappear or accumulate) and are so called bystander (or passenger) mutations. However, it is likely that combination of the different models takes place in a tumor. 


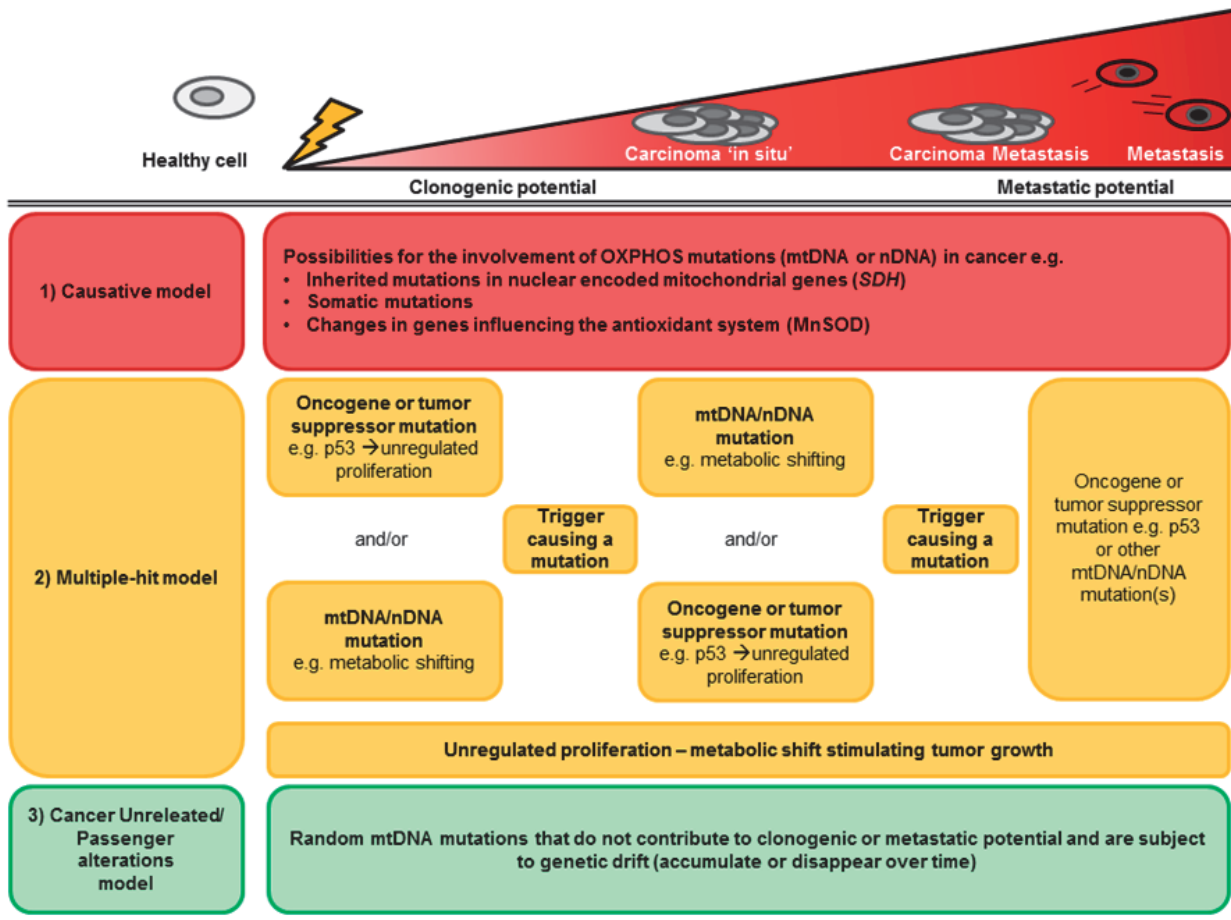

Figure 3. Representation of different scenarios possibly explaining how mitochondrial genes encoded in nDNA or mtDNA could contribute to cancer.

When a cell gains clonogenic potential and evolves into a primary tumor or metastasis, different alterations occur in nuclear encoded mitochondrial genes and mtDNA. Potentially three different scenarios could describe and explain these observations. In the causative model, mutations in mitochondrial genes encoded either in the nDNA or mtDNA cause the development of a carcinoma and/or metastasis. The multiple-hit model describes a scenario where different types of mutations (in classic oncogenes or tumor suppressor genes and in mtDNA or nDNA OXPHOS genes) together contribute to the clonogenic potential and the development of a carcinoma or metastasis. Mutations can follow sequentially or a driver mutation needs additional mutations after which it can express its mutagenic or metastatic potential resulting in a pathogenic phenotype. Additionally, also low heteroplasmic mtDNA mutation levels can be increased via drift. This increased mutation percentage could thereby exhibit the pathogenic effect of the mutation. The third scenario consists of passenger mutations observed in the tumor but not contributing to an increased clonogenic or metastatic potential. The mutations can accumulate or disappear over time (genetic-drift). All different models can exist in the same tumor site, tumor or tumor type.

\section{mtDNA and cancer treatment implications}

In cancer patients, the choice of therapy depends on multiple factors such as the histopathology of the tumor, the stage of the disease and the patient's condition. The three most commonly used treatment options, sometimes combined with one another, are surgery, chemotherapy and radiotherapy. 


\section{Chemotherapy}

mtDNA abnormalities affect the response and outcome of therapies in cancer patients. As described in a review by Singh et al. different studies have already shown that alterations in mtDNA can result in chemotherapy resistance [4]. It has been reported that mutations in the NADH dehydrogenase subunit 4 (MT-ND4) contributed to acquired chemoresistance during paclitaxel carboplatin treatment [165].

However, it also has been suggested that mtDNA variations can be induced by chemotherapy. In a case report a patient with ovarian carcinoma had a mtDNA mutation in the MT-ND4 gene (m.10875T $>C$ ) which occurred after chemotherapy treatment [165]. A second study observed that in a leukemia patient 6 months after chemotherapy more mtDNA variations were found compared to the samples withdrawn prior to treatment [8]. In the same study, leukemia patients treated with a fludarabine/alkylator-based chemotherapy regimen displayed an increase in mtDNA mutations in primary leukemic cells compared to non-treated patients [8]. Additionally, accumulation of mtDNA damage even persisted after ending doxorubicin treatment and was associated with adverse effects such as cardiotoxicity [166]. Therefore, it seems that de novo variations can be induced by chemotherapy. Another possibility is that due to selection a very low heteroplasmy mutation load could be increased in the patients due to its clonogenic advantageous property. Both possible mechanisms should be further investigated as well as the observations should be validated in larger study populations before any conclusions could be drawn.

Another chemotherapeutic drug, cisplatin, is able to accumulate in the mitochondria, causing impairment of mtDNA and mtRNA synthesis [167]. This drug has been shown in vitro to induce more adduct formation in the mtDNA compared to the nuclear DNA [168]. In addition, cisplatin leads to caspase-dependent apoptosis by changing the mitochondrial membrane permeability and resulting in the release of cytochrome $c$ into the cytosol, subsequently activating caspase 8 and 9 [169]. For the intestines, a correlation was observed between the mitochondrial density and cisplatin sensitivity. Cisplatin sensitivity was limited in normal ileum tissue having a low mitochondrial density, while the opposite was observed for the normal duodenum tissue, which has a high mitochondrial density. In line with these findings, in vitro cultured cells depleted from their mtDNA, rho-zero cells $\left(\rho^{0}\right.$, Fig. 2$)$ generated from normal intestinal epithelial cell lines (IEC-6) showed a four- to five-fold increased resistance against cisplatin compared to their parental counterparts [170]. This can be explained by the fact that cisplatin-induced adduct formation in mtDNA measured in head and neck squamous cell carcinoma cell lines can be up to 500 times higher compared to nDNA [171]. However, it has also been suggested that mtDNA mutations lead to an increased ROS production and that combination with chemotherapeutics with the same effect (like cisplatin) can lead to exhaustion of a cell's antioxidant capacity/response and thereby eventually lead 
to apoptosis [172-174]. Correlations of ROS production and sensitivity to 2methoxyestradiol, a ROS generating agent, have already been reported $[175,176]$

There seems to be some evidence that mtDNA variations could contribute to chemotherapy resistance and some drugs directly interact with the mtDNA. The observations also apply to radiotherapy, another conventional cancer therapy.

\section{Radiation therapy}

Ionizing radiation (IR) is a widely used cancer therapy that results in cellular damage in a direct or indirect cellular manner. The direct mechanism involves the transfer of energy from incident photons or particles to target molecules in their path. ROS are formed in the presence of oxygen, resulting in secondary damage [177]. Upon DNA damage, cells might undergo a temporary cell cycle arrest to repair the damage or die, mainly by mitotic catastrophe and only partly by apoptosis [178]; both processes require ATP $[179,180]$, and might involve directly or indirectly mitochondria as both are at least in part dependent on mitochondrial membrane permeabilization. This suggests that mitochondria are potentially involved in downstream irradiation effects [181, 182].

Studies have shown that $\rho^{0}$ cells are more radioresistant than parental cells containing wild-type mtDNA [183][184]. Human fibroblast and pancreatic cancer $\rho^{0}$ cells have shown higher survival rates following radiation, which could be related to decreased G2/M cell cycle arrest [183] or decreased apoptosis without alterations in cell cycle distribution [184]. Improved survival could not be linked to differences in antioxidant enzyme expression [183]. In contrast, other studies did not identify differences in survival after irradiation between human fibroblast and osteosarcoma in parental versus $\rho^{0}$ cells, although less micronuclei (fragments found in the cytoplasm which originates from nuclear DNA and correlates with dose/quality of irradiation [185]) formation was observed in $\rho^{0}$ cells after irradiation $[186,187]$.

A differential radiation response was observed between a normal B-lymphoblastoid cell line and the mitochondrial mutant cell lines for Leigh's syndrome (m.8993T>G mutation in MTATP6 ATP synthase gene) or Leber's optic atrophy (m.11778G>A mutation in MTND4 gene [188, 189]. In both cell lines, the apoptosis-related genes showed a remarkable up-regulation compared to the control cell line. However, the repair of the irradiation-induced double-stranded breaks was different for each of the mutant cell lines. Repair was significantly decreased in cells with Leigh's mutation, leading to worse short-term radiation survival, while for the Leber's cells, double-strand breaks could be repaired resulting in better short-term radiation survival although the studies were not performed in cell lines with the same background $[188,190]$. In a recently published in vivo study it was shown that irradiation of a SiHa cell line with induced mitochondrial dysfunction (showing a decrease of oxygen consumption upon exposure to ethidium 
bromide) lead to an increased time to reach the endpoint compared to their parental counterpart [191].

There are also indications that mtDNA variation may not only affect radiotherapy outcome, but that radiotherapy and chemotherapy itself also can induce mutations in the mtDNA. Wardell et al. observed an increased number of point mutations and deletions in patients treated with radiotherapy and chemotherapy [192]. Additionally, a decrease in mtDNA content has been reported as a result of radiotherapy in cancer patients [193]. However, another study found no evidence that radiotherapy for pediatric cancer, which resulted in scatter radiation to the ovaries, is associated with the mitochondrial genome mutation frequency in female cancer survivors and their children [194]. In conclusion, mtDNA mutations (induced by radiotherapy or germline) can also affect the response and therefore the outcome of radiotherapy although the precise role and mechanisms are not yet fully understood.

\section{Mitochondrial function interventions in cancer therapy}

ROS is one of the major processes implicated in cancer. It may be possible to reduce the ROS escalation caused by dysfunctional OXPHOS using metformin, a type II diabetes drug that is used to suppress gluconeogenesis and, among others, inhibits ROS production as well as mitochondrial complex I [195-197]. A previous study showed that type II diabetes patients have an increased risk of developing cancer with a poorer prognosis [198]. In 2005, Evans et al. observed a correlation between a reduced risk of developing cancer and treatment of type II diabetes patients with metformin [199], which was recently confirmed in a large cohort study [200].

Metformin activates adenosine monophosphate activated protein kinase (AMPK) and therefore stimulates muscles to take up glucose from the blood, a process also activated by exercise [199]. Furthermore, the activation of the cell metabolism regulator AMPK results in inhibition of $\mathrm{MTORC1}$ and its downstream signaling pathway, thereby decreasing protein synthesis and cell proliferation crucial for tumors [201, 202]. The combination of metformin and radiation therapy was shown to be successful in vitro since cellular survival was decreased [203]. Song et al. found that metformin was able to increase radiosensitivity of cancer cells in vitro and enhance radiation-induced growth delay of fibrosarcoma tumors as well as non-small cell lung cancer $[204,205]$. When metformin treatment was combined with 2-deoxyglucose (2-DG, a glucose analog), it resulted in an energetic stress cell death [206, 207]. Instead of using a glucose analog, glucose uptake could be inhibited by other substances (e.g. Pholertin, WZB117 or Fasentin) to enact a similar mechanism [208-210]. Inhibition of glucose uptake of the cell could also be a potential target for tumors with a dysfunctional OXPHOS system [211]. 
Both chemo- and radiotherapy could be influenced by mtDNA variations. Additionally, a combination therapy including conventional therapies and drugs influencing mitochondrial function could be explored as a therapeutic option for cancer patients.

\section{Opportunities and pitfalls in studies using mtDNA models}

Multiple studies have identified potential associations between mtDNA variations and cancer [64-73]. Mutations/deletions and copy number variations in the mtDNA are able to affect proper OXPHOS function and consequently change predisposition for a disorder. mtDNA variations (de novo, inherited, caused by ROS production in tumors or by cancer treatments) can promote tumor development and progression. Different mtDNA models are available for diagnostic and research purposes. Table 1 presents a short representation of in vitro, in vivo and ex vivo models.

\section{In vitro mtDNA models}

Effects of mtDNA depletion are commonly investigated in the $\rho^{0}$ cell model, mammalian cells depleted of mtDNA by exposure to ethidium bromide (Etbr) [212]. Consequently, $\rho^{0}$ cells rely completely on glycolysis instead of oxidative phosphorylation for energy production. Removal of Etbr leads to mtDNA repopulation and therefore it should be used throughout the entire experiment. Etbr treatment can lead to stalled proliferation [213], is genotoxic to cells in vitro (http://ntp.niehs.nih.gov) and can lead to off-target effects on the nDNA. Therefore, this model is not suitable for in vivo studies.

Most studies on pathophysiology have exploited fibroblasts derived from patients with mtDNA diseases or mutations. These models allow for high-throughput screening [77, $78,214]$ and testing of potential therapies in vitro. Unfortunately, the consequences of OXPHOS-related defects are sometimes less pronounced in in vitro fibroblast cultures, since cells are capable of switching from oxidative phosphorylation toward glycolysis under specific culturing conditions [215]. In addition, these cells are generally not able to form tumors by themselves. Therefore, fibroblasts are not suitable for investigating the link between cancer and mtDNA.

A cybrid cell line is another model used to study the effect of mtDNA variations in cancer. These are cell lines with the nuclear DNA background of a tumor cell line [134] and the mtDNA of another cell line (Fig. 2). The mtDNA can come from a patientderived cancer cell line, a fibroblast or a lymphocyte cell line. The main advantage of these cybrids is the ability to investigate whether phenotypes and biochemical changes are related to changes in the nDNA or mtDNA. Furthermore, cybrid models enable the investigation of in vitro and in vivo effects of different mtDNA mutations in cancer cell 
lines and tumors with the same nuclear background [216]. A possible disadvantage is the dependency on naturally occurring patient mtDNA variations.

\section{Mitochondrial animal models}

Patients with variations in their mtDNA are often unique, so grouping them is very difficult. Therefore, data obtained from patient studies should be carefully interpreted with respect to large inter-individual variation. The use of murine models is in this respect advantageous, as experimental settings can be better controlled than in patient studies. For instance, cell lines with the same nuclear genetic background harboring one mtDNA variation can be used as xenograft models. Additionally, to easier investigate the molecular mechanisms, specific mitochondrial murine models, such as the POLG mutator mouse, can be used either combined with mouse models which spontaneously develop cancer.

Currently, different mouse models are available to unravel the pathways involved in mtDNA mutations and cancer (Table 1). The most studied mouse model created for translational research in this particular field is the POLG knock-in mouse. These mice lack the proofreading function of pol $\gamma$ and therefore show an accumulation of mutations and deletions in their mtDNA [217].

In homozygous mice, but not in heterozygous mice, a premature aging phenotype resulting in effects such as heart enlargement and hearing impairment has been observed. However, no cancer development has been reported [217, 218]. Another mouse model carries a mutation in the complex I subunit NADH dehydrogenase ubiquinone Fe-S protein 4 (NDUFS4). These mice displayed a phenotype similar to that in human patients carrying a NDUFS4 mutation, and a full knockout was lethal at approximately seven weeks of age $[219,220]$. A heterozygote mutant of the NDUFS4 mice was viable and still showed the biochemical changes observed in patients suffering from Leigh's syndrome [221]. 
Involvement of mtDNA and mitochondrial dysfunction in cancer

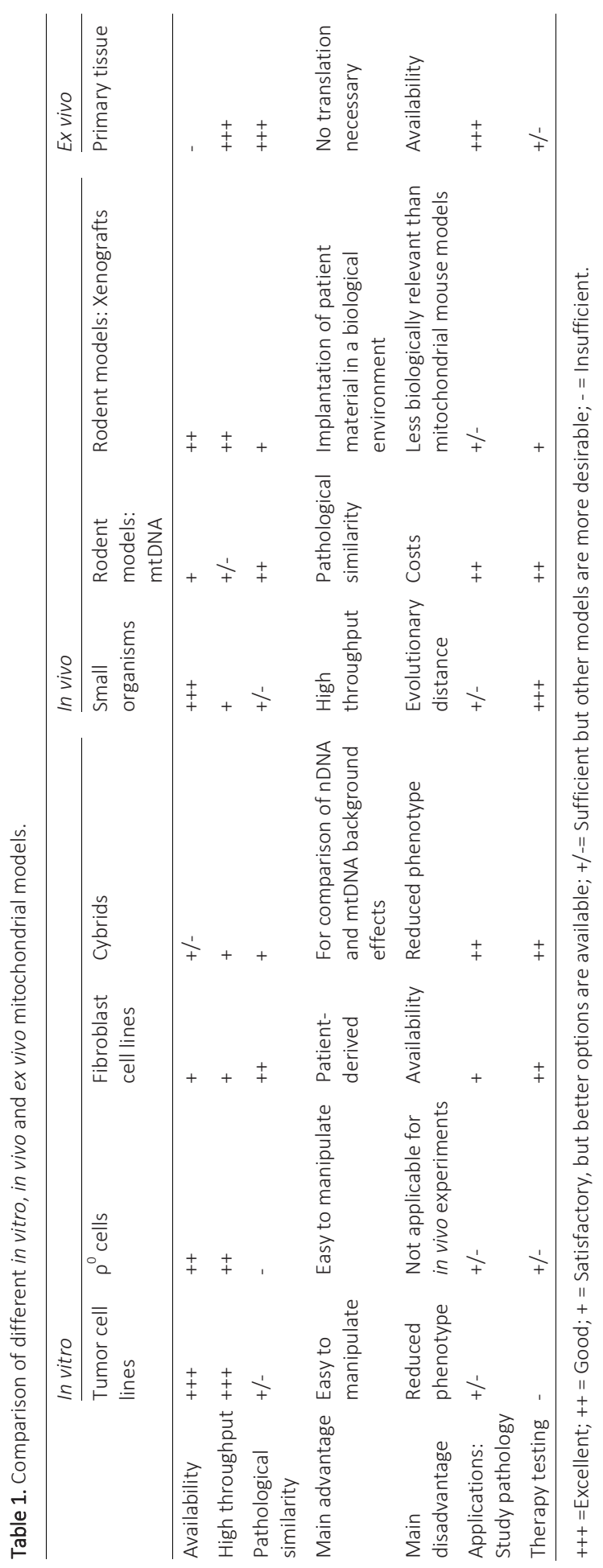


A third model, the thymidine phosphorylase (Tp) and uridine phosphorylase (Upp) double knockout mouse model $\left(T p^{-/} U p p-1^{-/-}\right)$has been developed for studying mitochondrial neurogastrointestinal encephalomyopathy (MNGIE). Murine UPP-1, unlike human UPP-1, cleaves thymidine as well as uridine; therefore, the full capacity to cleave mouse thymidine is abolished in this mouse model [222-224]. In addition, deficiencies of complex I and IV could be observed in the brain of very old $T p^{-/-} U p p-1^{-/-}$ mice [224]. This thymidine-induced dNTP imbalance has been recently demonstrated to be overcome by the metabolic switch into the salvage pathway in acute lymphoblastic leukemia cells, therefore avoiding the lethal replication stress and the subsequent tumor progression. Accordingly, strategies focused on inhibition of this switch have been suggested as metabolic intervention in acute lymphoblastic leukemia [225]. Tp is found to be overexpressed in different tumors (e.g. non-small-cell lung cancer and colorectal cancer) and seems to play an important role in angiogenesis, invasion and metastasis of tumors [226, 227]. A study by Moghaddam et al. found that Tp has angiogenic properties and, in a MCF7 breast cancer xenograft model, Tp overexpression led to faster tumor growth [228]. In contrast, low Tp expression correlates to the favorable prognosis of gastric cancer treated with chemotherapy [229, 230] and promoter methylation has been proposed as a mechanism of its expression down regulation in different cancer cells [231, 232].

Mice can be given a trigger (e.g. a toxin such as nitrosaminoketone) or have a nuclear gene background such as the K-rasLA mouse model to develop cancer [233, 234]. This in combination with a murine model with a pathological mtDNA background could lead to the creation of a model containing both the mtDNA phenotype and the cancer phenotype to investigate the contribution of both parameters to the phenotype of the tumor.

As every mouse strain has specific differences and it is labor intensive to make genetic mouse models for every human phenotype, another option is to use small animal models specially created for studying the mitochondrial DNA mutation pathology. Animal models, such as Danio rerio (zebra fish) [235], Drosophila melanogaster (fruit fly) [236] and Caenorhabditis elegans (round worm) [237], develop rather quickly and the model can be manipulated by directly adding compounds to the food, medium or water, or irradiating their culture dish. Because the evolutionary distance to humans is larger than for mice, some of these organisms do not contain all the organs affected in humans [238].

\section{OXPHOS inhibitors in cellular models for therapeutic interventions}

Several studies demonstrated that inhibition of glycolysis could be a possible treatment option for cancers with compromised mitochondrial function (see review Pelicano et al. [211]). As these cancers rely more on aerobic glycolysis (Warburg effect), driving the 
tumors to use OXPHOS instead would lead to sever energetic stress. Cells with mtDNA mutations or with a lack of oxygen (hypoxia) are not able to use alternative energy sources such as fatty acids and amino acids to produce metabolic intermediates which can be used in the Krebs cycle for ATP production through OXPHOS. In these cells inhibition of glycolysis could induce cell death $[239,240]$. For instance, in an in vitro system using human leukemia cells ( $\mathrm{HL}-60)$ and human lymphoma cells (Raji), cells with a respiration defect were less sensitive to cytostatic drugs like $1-\beta$ - $d$-arabinofuranosylcytosine (ara- $C$ ), doxorubicin (Adriamycin), taxol and vincristine [29]. However, lonidamine, 3bromopyruvate and 2-deoxyglucose are known inhibitors of glycolysis that showed promising results [29, 206, 207, 241]. In addition, partial inhibition of OXPHOS can be achieved by inhibition of the different complexes of the OXPHOS system. The pesticide rotenone is known for its ability to inhibit complex I [220]. It can be used in an experimental set-up at low concentrations, but it is not likely to obtain U.S. Food and Drug Administration (FDA) approval for usage in humans due to toxic effects (e.g. irritation of the respiratory tract and apoptosis of erythrocytes) [242, 243]. Metformin, an FDA approved drug prescribed to patients with type II diabetes [201], is another inhibitor of complex I [195]. However, as discussed above metformin is also used as an anti-cancer drug by inhibiting the mTOR signaling through activation of AMPK. A more potent drug for complex I inhibition is phenformin [244] a drug related to metformin from the biguanide class that is able to inhibit the development and growth of MCF7 and MDAMV231 tumors in a xenograft mouse model [245]. Additionally, it also affects the mTOR signaling pathway [246]. In xenograft melanomas and in genetically modified mice for melanomas (BRAF $^{\mathrm{V} 600 \mathrm{E}}$ ) combined therapy of phenformin and PLX4720 showed a significant growth reduction whereas treatment with only phenformin or PLX470 resulted in growth inhibition but no tumor regression [246]. However, FDA has redrawn phenformin from the North-American market in 1977, due to its association with fatal lactic acidosis in diabetic patients.

\section{Future prospects}

There is still a lot of debate about the precise relationship between mtDNA variants, OXPHOS abnormalities and cancer. Are these mtDNA variations driving disease, involved in disease progression or implicated in treatment response and adaptation to treatment? Or are they merely passenger observations?

\section{Detecting mtDNA mutations}

Various platforms can be used to identify mtDNA mutations in tumor material or other specimens. Using mutation specific restriction digestion or restriction fragment length polymorphism (RFLP) analysis, screening for common mtDNA mutations becomes 
possible [247]. Denaturing high performance liquid chromatography can be used to determine heteroplasmy mtDNA mutations [248]. Pyrosequencing allows accurate quantification of the heteroplasmy levels of a variation [249]. For detection of mtDNA deletions long-range PCR [250, 251] or quantitative real-time PCR based methods [252] and southern blotting [253] could be used. Though, currently there is more desire for high-throughput methods of which some of these are listed below.

Random Mutation Capture (RMC) assay is based on single molecule amplification which facilitates the user in measuring relative values of the spontaneous mutation frequency and mtDNA deletions [254]. Another method is digital deletion detection (3D assay) and is based on the RMC method; with $3 \mathrm{D}$ it is also possible to identify rare deletion events [255]. Currently, next generation sequencing (NGS) of mtDNA is the standard for determination of homo- and heteroplasmic variations. Heteroplasmy depends on tissue type; therefore somatic mutations in tumor tissue are mainly heteroplasmic since tumors exist out of different cell types. NGS has an enhanced sensitivity for detecting low levels of heteroplasmy and has as well an increased coverage [256]. The previously used platform,

MitoChip, is commonly used for the identification of homoplasmic mutations. Although the detection range is limited, also some heteroplastic variants can be found [257, 258]. mtDNA variants detected with Mitochip usually have to be confirmed using conventional Sanger sequencing.

\section{Identification of potential drug targets}

mtDNA research is not only pursuing the identification of variations of mtDNA that are involved in cancer and its treatment, but also the identification of potential drug targets. The ideal treatment would be to repair the appropriate defective mtDNA sequence, although this would be almost impossible for cancer-related somatic mtDNA mutations. Different strategies to achieve this goal have been suggested for severe germline mtDNA mutations. For example, blocking the replication of mutated mtDNA by peptide nucleic acid might result in repopulated cells with unaffected wild-type DNA [259]. However, as interventions specifically for mtDNA are difficult to implement, mitochondria and mtDNA can be targeted at multiple downstream steps of physiological processes. Drugs can interact with mitochondrial permeability, membrane potential, energy supply, antioxidants, ROS production and apoptosis. For instance, influencing the function of B-cell lymphoma 2 ( $\mathrm{Bcl}-2$ ), an anti-apoptotic protein, might be used as a target. Bcl-2 is often overexpressed in solid tumors and contributes to resistance to conventional therapies [260]. Possible targets in the cell are the mitochondrial outer membrane proteins (e.g. monocarboxylate transporter 1 (MCT1) and mitochondrial pyruvate carriers (MPCs)) or inner membrane proteins (complex I, complex II, complex III, complex IV and complex V), the ETC and the Krebs cycle. 
Alternatively, variations in mtDNA can be exploited to tailor patients' therapy. It is unlikely that all patients with different variations causing OXPHOS deficiency will benefit from the same treatment. To illustrate, for tumors with heteroplasmic driver mutations interventions that stimulate mtDNA quantity can result in high mutation levels and should be avoided to prevent that tumors become more therapy resistant. In this case, stimulating mitophagy could be a better option to eliminate the mutation. Altogether, personalized treatments should be carefully evaluated in respect to the pathological process in the tumors of the cancer patients.

\section{Conclusions}

Over the last decade, the role of mitochondria and, more specifically, mtDNA in cancer has started to be explored. Different signaling processes and ROS production have been implicated in the development and progression of cancer, and these processes can be affected by changes in the mtDNA. However, the exact molecular mechanisms are not yet known. The link between mtDNA and different processes (DNA damage, ROS and apoptosis) and how this plays a role in cancer remains especially vague and is not well defined. In this review, we discussed 3 different types of models (Fig. 3) of nuclear encoded and mitochondrial encoded DNA mutations and how they relate to cancer and cancer therapy. The mutations can have a causative effect, could depended on the multiple hit model or can be just neutral the so-called passenger mutations. Although a combination of these models could occur in the tumor.

Future research should focus on establishing whether these processes are causes or consequences and under which circumstances, and exploring whether they are connected or unrelated to changes in the mtDNA. Upon progressive knowledge about the role of mtDNA, it will be possible to better understand cancer initiation and progression and to improve the prognosis and treatment of patients.

\section{Conflict of interest statement}

There are no conflicts of interest.

\section{Acknowledgements}

We would like to acknowledge the financial support of the Center for Translational and Molecular Medicine (AIRFORCE), METOXIA (Metoxia project ref. 2008-222741), the Netherlands Genomics Initiative (pre-seed grant 2012, 93612005), the azM 
Chapter 2

onderzoeksfonds (2012-2014) and the Sophia Foundation (SSW0645). This study was conducted with the support of the Ontario Institute for Cancer Research to PCB through funding provided by the Government of Ontario. 


\section{References}

1. Anderson, S., et al., Sequence and organization of the human mitochondrial genome. Nature, 1981. 290(5806): p. 457-65.

2. Smeitink, J., L. van den Heuvel, and S. DiMauro, The genetics and pathology of oxidative phosphorylation. Nature reviews. Genetics, 2001. 2(5): p. 342-52.

3. St-Pierre, J., et al., Topology of superoxide production from different sites in the mitochondrial electron transport chain. The Journal of biological chemistry, 2002. 277(47): p. 44784-90.

4. Singh, K.K., et al., Mitochondrial DNA determines the cellular response to cancer therapeutic agents. Oncogene, 1999. 18(48): p. 6641-6.

5. Voets, A.M., et al., Transcriptional changes in OXPHOS complex I deficiency are related to anti-oxidant pathways and could explain the disturbed calcium homeostasis. Biochimica et biophysica acta, 2012. 1822(7): p. 1161-1168

6. Voets, A.M., et al., Patient-derived fibroblasts indicate oxidative stress status and may justify antioxidant therapy in OXPHOS disorders. Biochimica et biophysica acta, 2012. 1817(11): p. 1971-8.

7. Porporato, P.E., et al., A Mitochondrial Switch Promotes Tumor Metastasis. Cell reports, 2014. 8(3): p. 754-766.

8. Carew, J.S., et al., Mitochondrial DNA mutations in primary leukemia cells after chemotherapy: clinical significance and therapeutic implications. Leukemia : official journal of the Leukemia Society of America, Leukemia Research Fund, U.K, 2003. 17(8): p. 1437-47.

9. McCord, J.M. and I. Fridovich, Superoxide dismutase. An enzymic function for erythrocuprein (hemocuprein). The Journal of biological chemistry, 1969. 244(22): p. 6049-55.

10. Wispe, J.R., et al., Synthesis and processing of the precursor for human mangano-superoxide dismutase. Biochimica et biophysica acta, 1989. 994(1): p. 30-6.

11. Oberley, L.W. and G.R. Buettner, Role of superoxide dismutase in cancer: a review. Cancer research, 1979. 39(4): p. 1141-9.

12. Oberley, L.W., Anticancer therapy by overexpression of superoxide dismutase. Antioxidants \& redox signaling, 2001. 3(3): p. 461-72.

13. Miriyala, S., et al., Manganese superoxide dismutase, MnSOD and its mimics. Biochimica et biophysica acta, 2012. 1822(5): p. 794-814.

14. $\mathrm{Xu}, \mathrm{Y}$., et al., Mutations in the promoter reveal a cause for the reduced expression of the human manganese superoxide dismutase gene in cancer cells. Oncogene, 1999. 18(1): p. 93-102.

15. Hwang, C., A.J. Sinskey, and H.F. Lodish, Oxidized redox state of glutathione in the endoplasmic reticulum. Science, 1992. 257(5076): p. 1496-502.

16. Karplus, P.A. and G.E. Schulz, Refined structure of glutathione reductase at 1.54 A resolution. Journal of molecular biology, 1987. 195(3): p. 701-29.

17. Lin, M.T. and M.F. Beal, Mitochondrial dysfunction and oxidative stress in neurodegenerative diseases. Nature, 2006. 443(7113): p. 787-95.

18. Warburg, O., F. Wind, and E. Negelein, The Metabolism of Tumors in the Body. The Journal of general physiology, 1927. 8(6): p. 519-30.

19. Rempel, A., et al., Glucose catabolism in cancer cells: amplification of the gene encoding type II hexokinase. Cancer research, 1996. 56(11): p. 2468-71.

20. Sharma, L.K., et al., Mitochondrial respiratory complex I dysfunction promotes tumorigenesis through ROS alteration and AKT activation. Human molecular genetics, 2011. 20(23): p. 4605-16.

21. Geromel, V., et al., Superoxide-induced massive apoptosis in cultured skin fibroblasts harboring the neurogenic ataxia retinitis pigmentosa (NARP) mutation in the ATPase-6 gene of the mitochondrial DNA. Human molecular genetics, 2001. 10(11): p. 1221-8.

22. Wallace, D.C., A mitochondrial paradigm of metabolic and degenerative diseases, aging, and cancer: a dawn for evolutionary medicine. Annual review of genetics, 2005. 39: p. 359-407. 
23. Kokoszka, J.E., et al., Increased mitochondrial oxidative stress in the Sod2 (+/-) mouse results in the agerelated decline of mitochondrial function culminating in increased apoptosis. Proceedings of the National Academy of Sciences of the United States of America, 2001. 98(5): p. 2278-83.

24. Trifunovic, A., et al., Somatic mtDNA mutations cause aging phenotypes without affecting reactive oxygen species production. Proceedings of the National Academy of Sciences of the United States of America, 2005. 102(50): p. 17993-8.

25. Wallace, D.C., Mitochondria and cancer. Nature reviews. Cancer, 2012. 12(10): p. 685-98.

26. Gogvadze, V., B. Zhivotovsky, and S. Orrenius, The Warburg effect and mitochondrial stability in cancer cells. Molecular aspects of medicine, 2010. 31(1): p. 60-74.

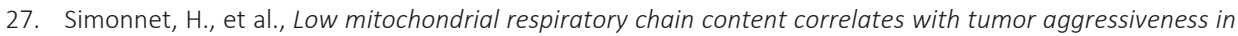
renal cell carcinoma. Carcinogenesis, 2002. 23(5): p. 759-68.

28. Wu, M., et al., Multiparameter metabolic analysis reveals a close link between attenuated mitochondrial bioenergetic function and enhanced glycolysis dependency in human tumor cells. Am J Physiol Cell Physiol, 2007. 292(1): p. C125-36.

29. Xu, R.H., et al., Inhibition of glycolysis in cancer cells: a novel strategy to overcome drug resistance associated with mitochondrial respiratory defect and hypoxia. Cancer research, 2005. 65(2): p. 613-21.

30. Choo, A.Y., et al., Glucose addiction of TSC null cells is caused by failed mTORC1-dependent balancing of metabolic demand with supply. Molecular cell, 2010. 38(4): p. 487-99.

31. Zaugg, K., et al., Carnitine palmitoyltransferase $1 \mathrm{C}$ promotes cell survival and tumor growth under conditions of metabolic stress. Genes \& development, 2011. 25(10): p. 1041-51.

32. Whitaker-Menezes, D., et al., Hyperactivation of oxidative mitochondrial metabolism in epithelial cancer cells in situ: visualizing the therapeutic effects of metformin in tumor tissue. Cell cycle, 2011. 10(23): p. 4047-64.

33. Rodriguez-Enriquez, S., et al., Oxidative phosphorylation is impaired by prolonged hypoxia in breast and possibly in cervix carcinoma. The international journal of biochemistry \& cell biology, 2010. 42(10): p. 1744-51.

34. Park, J., et al., Leptin receptor signaling supports cancer cell metabolism through suppression of mitochondrial respiration in vivo. The American journal of pathology, 2010. 177(6): p. 3133-44.

35. van den Heuvel, L. and J. Smeitink, The oxidative phosphorylation (OXPHOS) system: nuclear genes and human genetic diseases. BioEssays : news and reviews in molecular, cellular and developmental biology, 2001. 23(6): p. 518-25.

36. Vander Heiden, M.G., L.C. Cantley, and C.B. Thompson, Understanding the Warburg effect: the metabolic requirements of cell proliferation. Science, 2009. 324(5930): p. 1029-33.

37. Polyak, K., et al., A model for p53-induced apoptosis. Nature, 1997. 389(6648): p. 300-5.

38. Mercer, W.E., et al., Negative growth regulation in a glioblastoma tumor cell line that conditionally expresses human wild-type p53. Proceedings of the National Academy of Sciences of the United States of America, 1990. 87(16): p. 6166-70.

39. Tanaka, H., et al., A ribonucleotide reductase gene involved in a p53-dependent cell-cycle checkpoint for DNA damage. Nature, 2000. 404(6773): p. 42-9.

40. Bayley, J.P., P. Devilee, and P.E. Taschner, The SDH mutation database: an online resource for succinate dehydrogenase sequence variants involved in pheochromocytoma, paraganglioma and mitochondrial complex I/ deficiency. BMC medical genetics, 2005. 6: p. 39.

41. Baysal, B.E., et al., Mutations in SDHD, a mitochondrial complex /l gene, in hereditary paraganglioma. Science, 2000. 287(5454): p. 848-51.

42. Niemann, S. and U. Muller, Mutations in SDHC cause autosomal dominant paraganglioma, type 3. Nature genetics, 2000. 26(3): p. 268-70.

43. Ishii, T., et al., A mutation in the SDHC gene of complex II increases oxidative stress, resulting in apoptosis and tumorigenesis. Cancer research, 2005. 65(1): p. 203-9.

44. Dwight, T., et al., Familial SDHA mutation associated with pituitary adenoma and pheochromocytoma/paraganglioma. The Journal of clinical endocrinology and metabolism, 2013. 98(6): p. E1103-8. 
45. Ricketts, C., et al., Germline SDHB mutations and familial renal cell carcinoma. Journal of the National Cancer Institute, 2008. 100(17): p. 1260-2.

46. Janeway, K.A., et al., Defects in succinate dehydrogenase in gastrointestinal stromal tumors lacking KIT and PDGFRA mutations. Proceedings of the National Academy of Sciences of the United States of America, 2011. 108(1): p. 314-8.

47. Kim, S., et al., Succinate dehydrogenase expression in breast cancer. SpringerPlus, 2013. 2(1): p. 299.

48. Tomlinson, I.P., et al., Germline mutations in FH predispose to dominantly inherited uterine fibroids, skin leiomyomata and papillary renal cell cancer. Nature genetics, 2002. 30(4): p. 406-10.

49. Lehtonen, R., et al., Biallelic inactivation of fumarate hydratase (FH) occurs in nonsyndromic uterine leiomyomas but is rare in other tumors. The American journal of pathology, 2004. 164(1): p. 17-22.

50. Lehtonen, H.J., et al., Conventional renal cancer in a patient with fumarate hydratase mutation. Human pathology, 2007. 38(5): p. 793-6.

51. Mardis, E.R., et al., Recurring mutations found by sequencing an acute myeloid leukemia genome. The New England journal of medicine, 2009. 361(11): p. 1058-66.

52. Paschka, P., et al., IDH1 and IDH2 mutations are frequent genetic alterations in acute myeloid leukemia and confer adverse prognosis in cytogenetically normal acute myeloid leukemia with NPM1 mutation without FLT3 internal tandem duplication. Journal of clinical oncology : official journal of the American Society of Clinical Oncology, 2010. 28(22): p. 3636-43.

53. Cairns, R.A., et al., IDH2 mutations are frequent in angioimmunoblastic T-cell lymphoma. Blood, 2012. 119(8): p. 1901-3.

54. Amary, M.F., et al., IDH1 and IDH2 mutations are frequent events in central chondrosarcoma and central and periosteal chondromas but not in other mesenchymal tumours. The Journal of pathology, 2011. 224(3): p. 334-43.

55. Sjoblom, T., et al., The consensus coding sequences of human breast and colorectal cancers. Science, 2006. 314(5797): p. 268-74.

56. Parsons, D.W., et al., An integrated genomic analysis of human glioblastoma multiforme. Science, 2008. 321(5897): p. 1807-12.

57. Yan, H., et al., IDH1 and IDH2 mutations in gliomas. The New England journal of medicine, 2009. 360(8): p. 765-73.

58. Taylor, B.S., et al., Integrative genomic profiling of human prostate cancer. Cancer cell, 2010. 18(1): p. 1122.

59. Gaude, E. and C. Frezza, Defects in mitochondrial metabolism and cancer. Cancer \& metabolism, 2014. 2: p. 10.

60. Selak, M.A., et al., Succinate links TCA cycle dysfunction to oncogenesis by inhibiting HIF-alpha prolyl hydroxylase. Cancer cell, 2005. 7(1): p. 77-85.

61. Zimmermann, F.A., et al., Lack of complex I is associated with oncocytic thyroid tumours. British journal of cancer, 2009. 100(9): p. 1434-7.

62. Pereira, L., et al., Somatic mitochondrial DNA mutations in cancer escape purifying selection and high pathogenicity mutations lead to the oncocytic phenotype: pathogenicity analysis of reported somatic mtDNA mutations in tumors. BMC cancer, 2012. 12: p. 53.

63. Kulawiec, M., et al., Tumorigenic transformation of human breast epithelial cells induced by mitochondrial DNA depletion. Cancer biology \& therapy, 2008. 7(11): p. 1732-43.

64. Parrella, P., et al., Detection of mitochondrial DNA mutations in primary breast cancer and fine-needle aspirates. Cancer research, 2001. 61(20): p. 7623-6.

65. Hosgood, H.D., 3rd, et al., Mitochondrial DNA copy number and lung cancer risk in a prospective cohort study. Carcinogenesis, 2010. 31(5): p. 847-9.

66. Jakupciak, J.P., et al., Performance of mitochondrial DNA mutations detecting early stage cancer. BMC cancer, 2008. 8: p. 285.

67. Lam, E.T., et al., Mitochondrial DNA Sequence Variation and Risk of Pancreatic Cancer. Cancer research, 2012. 
68. Petros, J.A., et al., mtDNA mutations increase tumorigenicity in prostate cancer. Proceedings of the National Academy of Sciences of the United States of America, 2005. 102(3): p. 719-24.

69. Liu, V.W., et al., High incidence of somatic mitochondrial DNA mutations in human ovarian carcinomas. Cancer research, 2001. 61(16): p. 5998-6001.

70. Polyak, K., et al., Somatic mutations of the mitochondrial genome in human colorectal tumours. Nature genetics, 1998. 20(3): p. 291-3.

71. Fliss, M.S., et al., Facile detection of mitochondrial DNA mutations in tumors and bodily fluids. Science, 2000. 287(5460): p. 2017-9.

72. Nishikawa, M., et al., Somatic mutation of mitochondrial DNA in cancerous and noncancerous liver tissue in individuals with hepatocellular carcinoma. Cancer research, 2001. 61(5): p. 1843-5.

73. Dasgupta, S., et al., Mitochondrial DNA mutations in respiratory complex-l in never-smoker lung cancer patients contribute to lung cancer progression and associated with EGFR gene mutation. Journal of cellular physiology, 2012. 227(6): p. 2451-60.

74. Boultwood, J., et al., Amplification of mitochondrial DNA in acute myeloid leukaemia. British journal of haematology, 1996. 95(2): p. 426-31.

75. Richter, C., J.W. Park, and B.N. Ames, Normal oxidative damage to mitochondrial and nuclear DNA is extensive. Proceedings of the National Academy of Sciences of the United States of America, 1988. 85(17): p. 6465-7.

76. DiMauro, S. and E.A. Schon, Mitochondrial DNA mutations in human disease. American journal of medical genetics, 2001. 106(1): p. 18-26.

77. van der Westhuizen, F.H., et al., Human mitochondrial complex / deficiency: investigating transcriptional responses by microarray. Neuropediatrics, 2003. 34(1): p. 14-22.

78. Valsecchi, F., et al., Complex I disorders: causes, mechanisms, and development of treatment strategies at the cellular level. Developmental disabilities research reviews, 2010. 16(2): p. 175-82.

79. Larsson, N.G. and D.A. Clayton, Molecular genetic aspects of human mitochondrial disorders. Annual review of genetics, 1995. 29: p. 151-78.

80. Chinnery, P.F. and D.C. Samuels, Relaxed replication of mtDNA: A model with implications for the expression of disease. American journal of human genetics, 1999. 64(4): p. 1158-65.

81. Lynch, M., B. Koskella, and S. Schaack, Mutation pressure and the evolution of organelle genomic architecture. Science, 2006. 311(5768): p. 1727-30.

82. Johnson, A.A. and K.A. Johnson, Exonuclease proofreading by human mitochondrial DNA polymerase. The Journal of biological chemistry, 2001. 276(41): p. 38097-107.

83. Kornberg, R.D., Chromatin structure: a repeating unit of histones and DNA. Science, 1974. 184(4139): p. 868-71.

84. Yakes, F.M. and B. Van Houten, Mitochondrial DNA damage is more extensive and persists longer than nuclear DNA damage in human cells following oxidative stress. Proceedings of the National Academy of Sciences of the United States of America, 1997. 94(2): p. 514-9.

85. Cullis, P.M., et al., Electron transfer from protein to DNA in irradiated chromatin. Nature, 1987. 330(6150): p. 773-4.

86. Liang, Q. and P.C. Dedon, CU(II)/H2O2-induced DNA damage is enhanced by packaging of DNA as a nucleosome. Chemical research in toxicology, 2001. 14(4): p. 416-22.

87. Alam, T.I., et al., Human mitochondrial DNA is packaged with TFAM. Nucleic acids research, 2003. 31(6): p. 1640-5.

88. Guliaeva, N.A., E.A. Kuznetsova, and A.I. Gaziev, [Proteins associated with mitochondrial DNA protect it against the action of X-rays and hydrogen peroxide]. Biofizika, 2006. 51(4): p. 692-7.

89. Davis, A.F., et al., Mitochondrial DNA polymerase gamma is expressed and translated in the absence of mitochondrial DNA maintenance and replication. Nucleic acids research, 1996. 24(14): p. 2753-9.

90. Rao, J.U., et al., Genotype-specific abnormalities in mitochondrial function associate with distinct profiles of energy metabolism and catecholamine content in pheochromocytoma and paraganglioma. Clinical cancer research : an official journal of the American Association for Cancer Research, 2013. 19(14): p. 3787-95. 
91. de Groof, A.J., et al., Increased OXPHOS activity precedes rise in glycolytic rate in H-RasV12/E1A transformed fibroblasts that develop a Warburg phenotype. Molecular cancer, 2009. 8: p. 54.

92. Spelbrink, J.N., et al., In vivo functional analysis of the human mitochondrial DNA polymerase POLG expressed in cultured human cells. The Journal of biological chemistry, 2000. 275(32): p. 24818-28.

93. Naviaux, R.K. and K.V. Nguyen, POLG mutations associated with Alpers syndrome and mitochondrial DNA depletion. Annals of neurology, 2005. 58(3): p. 491.

94. Ekstrand, M.I., et al., Mitochondrial transcription factor A regulates mtDNA copy number in mammals. Human molecular genetics, 2004. 13(9): p. 935-44.

95. Guo, J., et al., Frequent truncating mutation of TFAM induces mitochondrial DNA depletion and apoptotic resistance in microsatellite-unstable colorectal cancer. Cancer research, 2011. 71(8): p. 2978-87.

96. Larsson, N.G., et al., Mitochondrial transcription factor A is necessary for mtDNA maintenance and embryogenesis in mice. Nature genetics, 1998. 18(3): p. 231-6.

97. Vermulst, M., et al., Mitochondrial point mutations do not limit the natural lifespan of mice. Nature genetics, 2007. 39(4): p. 540-3.

98. Vermulst, M., et al., DNA deletions and clonal mutations drive premature aging in mitochondrial mutator mice. Nature genetics, 2008. 40(4): p. 392-4.

99. Li, H., et al., Genetic modification of survival in tissue-specific knockout mice with mitochondrial cardiomyopathy. Proceedings of the National Academy of Sciences of the United States of America, 2000. 97(7): p. 3467-72.

100. Wang, J., et al., Dilated cardiomyopathy and atrioventricular conduction blocks induced by heart-specific inactivation of mitochondrial DNA gene expression. Nature genetics, 1999. 21(1): p. 133-7.

101. Balliet, R.M., et al., Mitochondrial oxidative stress in cancer-associated fibroblasts drives lactate production, promoting breast cancer tumor growth: understanding the aging and cancer connection. Cell cycle, 2011. 10(23): p. 4065-73.

102. Gomes, L.C., G. Di Benedetto, and L. Scorrano, During autophagy mitochondria elongate, are spared from degradation and sustain cell viability. Nature cell biology, 2011. 13(5): p. 589-98.

103. Falkenberg, M., N.G. Larsson, and C.M. Gustafsson, DNA replication and transcription in mammalian mitochondria. Annual review of biochemistry, 2007. 76: p. 679-99.

104. Ono, T., et al., Human cells are protected from mitochondrial dysfunction by complementation of DNA products in fused mitochondria. Nature genetics, 2001. 28(3): p. 272-5.

105. Youle, R.J. and D.P. Narendra, Mechanisms of mitophagy. Nat Rev Mol Cell Biol, 2011. 12(1): p. 9-14.

106. Gilkerson, R.W., et al., Mitochondrial autophagy in cells with mtDNA mutations results from synergistic loss of transmembrane potential and mTORC1 inhibition. Human molecular genetics, 2012. 21(5): p. 97890.

107. Degtyarev, M., et al., Akt inhibition promotes autophagy and sensitizes PTEN-null tumors to lysosomotropic agents. The Journal of cell biology, 2008. 183(1): p. 101-16.

108. Twig, G. and O.S. Shirihai, The interplay between mitochondrial dynamics and mitophagy. Antioxidants \& redox signaling, 2011. 14(10): p. 1939-51.

109. Mishmar, D., et al., Natural selection shaped regional mtDNA variation in humans. Proceedings of the National Academy of Sciences of the United States of America, 2003. 100(1): p. 171-6.

110. Wallace, D.C. and W. Fan, Energetics, epigenetics, mitochondrial genetics. Mitochondrion, 2010. 10(1): p. 12-31.

111. Smolkova, K., et al., Waves of gene regulation suppress and then restore oxidative phosphorylation in cancer cells. The international journal of biochemistry \& cell biology, 2011. 43(7): p. 950-68.

112. Torroni, A., et al., Classification of European mtDNAs from an analysis of three European populations. Genetics, 1996. 144(4): p. 1835-50.

113. Johnson, M.J., et al., Radiation of human mitochondria DNA types analyzed by restriction endonuclease cleavage patterns. Journal of molecular evolution, 1983. 19(3-4): p. 255-71.

114. Verma, M., et al., Meeting report: mitochondrial DNA and cancer epidemiology. Cancer research, 2007. 67(2): p. 437-9. 
115. Booker, L.M., et al., North American white mitochondrial haplogroups in prostate and renal cancer. The Journal of urology, 2006. 175(2): p. 468-72; discussion 472-3.

116. Canter, J.A., A.R. Kallianpur, and J.H. Fowke, Re: North American white mitochondrial haplogroups in prostate and renal cancer. The Journal of urology, 2006. 176(5): p. 2308-9; author reply 2309.

117. Bai, R.K., et al., Mitochondrial genetic background modifies breast cancer risk. Cancer research, 2007. 67(10): p. 4687-94.

118. van Oven, M. and M. Kayser, Updated comprehensive phylogenetic tree of global human mitochondrial DNA variation. Human mutation, 2009. 30(2): p. E386-94.

119. Andrews, R.M., et al., Reanalysis and revision of the Cambridge reference sequence for human mitochondrial DNA. Nat Genet, 1999. 23(2): p. 147.

120. Canter, J.A., et al., Mitochondrial DNA G10398A polymorphism and invasive breast cancer in AfricanAmerican women. Cancer research, 2005. 65(17): p. 8028-33.

121. Darvishi, K., et al., Mitochondrial DNA G10398A polymorphism imparts maternal Haplogroup $N$ a risk for breast and esophageal cancer. Cancer letters, 2007. 249(2): p. 249-55.

122. Francis, A., et al., A mitochondrial DNA variant 10398G>A in breast cancer among South Indians: an original study with meta-analysis. Mitochondrion, 2013. 13(6): p. 559-65.

123. Mosquera-Miguel, A., et al., Is mitochondrial DNA variation associated with sporadic breast cancer risk? Cancer research, 2008. 68(2): p. 623-5; author reply 624.

124. Salas, A., et al., The saga of the many studies wrongly associating mitochondrial DNA with breast cancer. BMC cancer, 2014. 14: p. 659.

125. Wang, L., et al., Mitochondrial genetic polymorphisms and pancreatic cancer risk. Cancer epidemiology, biomarkers \& prevention : a publication of the American Association for Cancer Research, cosponsored by the American Society of Preventive Oncology, 2007. 16(7): p. 1455-9.

126. Navaglia, F., et al., Mitochondrial DNA D-loop in pancreatic cancer: somatic mutations are epiphenomena while the germline $16519 \mathrm{~T}$ variant worsens metabolism and outcome. American journal of clinical pathology, 2006. 126(4): p. 593-601.

127. Halfdanarson, T.R., et al., Mitochondrial genetic polymorphisms do not predict survival in patients with pancreatic cancer. Cancer epidemiology, biomarkers \& prevention : a publication of the American Association for Cancer Research, cosponsored by the American Society of Preventive Oncology, 2008. 17(9): p. 2512-3.

128. Price, A.L., et al., Principal components analysis corrects for stratification in genome-wide association studies. Nature genetics, 2006. 38(8): p. 904-9.

129. Gochhait, S., et al., Concomitant presence of mutations in mitochondrial genome and p53 in cancer development - a study in north Indian sporadic breast and esophageal cancer patients. International journal of cancer. Journal international du cancer, 2008. 123(11): p. 2580-6.

130. He, L., et al., Somatic mitochondrial DNA mutations in adult-onset leukaemia. Leukemia, 2003. 17(12): p. 2487-91.

131. Jin, X., et al., Relationship between mitochondrial DNA mutations and clinical characteristics in human lung cancer. Mitochondrion, 2007. 7(5): p. 347-53.

132. Gasparre, G., et al., Disruptive mitochondrial DNA mutations in complex I subunits are markers of oncocytic phenotype in thyroid tumors. Proceedings of the National Academy of Sciences of the United States of America, 2007. 104(21): p. 9001-6.

133. Nagy, A., et al., Somatic mitochondrial DNA mutations in human chromophobe renal cell carcinomas. Genes, chromosomes \& cancer, 2002. 35(3): p. 256-60.

134. Ishikawa, K., et al., ROS-generating mitochondrial DNA mutations can regulate tumor cell metastasis. Science, 2008. 320(5876): p. 661-4.

135. Larman, T.C., et al., Spectrum of somatic mitochondrial mutations in five cancers. Proceedings of the National Academy of Sciences of the United States of America, 2012. 109(35): p. 14087-91.

136. Calabrese, C., et al., Respiratory complex I is essential to induce a Warburg profile in mitochondriadefective tumor cells. Cancer \& metabolism, 2013. 1(1): p. 11. 
137. Iommarini, L., et al., Different mtDNA mutations modify tumor progression in dependence of the degree of respiratory complex I impairment. Human molecular genetics, 2014. 23(6): p. 1453-1466.

138. Gasparre, G., et al., A mutation threshold distinguishes the antitumorigenic effects of the mitochondrial gene MTND1, an oncojanus function. Cancer research, 2011. 71(19): p. 6220-9.

139. Akgul, E.O., et al., MtDNA depletions and deletions may also be important in pathogenesis of lung cancer. Respiratory medicine, 2013. 107(11): p. 1814.

140. Yu, M., Generation, function and diagnostic value of mitochondrial DNA copy number alterations in human cancers. Life sciences, 2011. 89(3-4): p. 65-71.

141. Shen, J., et al., Mitochondrial copy number and risk of breast cancer: a pilot study. Mitochondrion, 2010. 10(1): p. 62-8.

142. Hofmann, J.N., et al., A nested case-control study of leukocyte mitochondrial DNA copy number and renal cell carcinoma in the Prostate, Lung, Colorectal and Ovarian Cancer Screening Trial. Carcinogenesis, 2014.

143. Wang, Y., et al., The increase of mitochondrial DNA content in endometrial adenocarcinoma cells: a quantitative study using laser-captured microdissected tissues. Gynecologic oncology, 2005. 98(1): p. 104-10.

144. Marucci, G., et al., Oncocytic glioblastoma: a glioblastoma showing oncocytic changes and increased mitochondrial DNA copy number. Human pathology, 2013. 44(9): p. 1867-76.

145. Jiang, W.W., et al., Increased mitochondrial DNA content in saliva associated with head and neck cancer. Clinical cancer research : an official journal of the American Association for Cancer Research, 2005. 11(7): p. 2486-91.

146. Wang, Y., et al., Association of decreased mitochondrial DNA content with ovarian cancer progression. British journal of cancer, 2006. 95(8): p. 1087-91.

147. Chen, T., et al., The mitochondrial DNA 4,977-bp deletion and its implication in copy number alteration in colorectal cancer. BMC medical genetics, 2011. 12: p. 8.

148. Lin, C.S., et al., The role of mitochondrial DNA alterations in esophageal squamous cell carcinomas. The Journal of thoracic and cardiovascular surgery, 2010. 139(1): p. 189-197 e4.

149. Vivekanandan, P., et al., Mitochondrial mutations in hepatocellular carcinomas and fibrolamellar carcinomas. Modern pathology : an official journal of the United States and Canadian Academy of Pathology, Inc, 2010. 23(6): p. 790-8.

150. Mizumachi, T., et al., Increased distributional variance of mitochondrial DNA content associated with prostate cancer cells as compared with normal prostate cells. The Prostate, 2008. 68(4): p. 408-17.

151. Mambo, E., et al., Tumor-specific changes in mtDNA content in human cancer. International journal of cancer. Journal international du cancer, 2005. 116(6): p. 920-4.

152. Lee, H.C., et al., Increase of mitochondria and mitochondrial DNA in response to oxidative stress in human cells. The Biochemical journal, 2000. 348 Pt 2: p. 425-32.

153. Correia, R.L., et al., Mitochondrial DNA depletion and its correlation with TFAM, TFB1M, TFB2M and POLG in human diffusely infiltrating astrocytomas. Mitochondrion, 2011. 11(1): p. 48-53.

154. Yu, M., Y. Wan, and Q. Zou, Decreased copy number of mitochondrial DNA in Ewing's sarcoma. Clinica chimica acta; international journal of clinical chemistry, 2010. 411(9-10): p. 679-83.

155. Dai, J.G., et al., Mitochondrial genome microsatellite instability and copy number alteration in lung carcinomas. Asian Pacific journal of cancer prevention : APJCP, 2013. 14(4): p. 2393-9.

156. Wu, C.W., et al., Mitochondrial DNA mutations and mitochondrial DNA depletion in gastric cancer. Genes, chromosomes \& cancer, 2005. 44(1): p. 19-28.

157. Meierhofer, D., et al., Decrease of mitochondrial DNA content and energy metabolism in renal cell carcinoma. Carcinogenesis, 2004. 25(6): p. 1005-10.

158. Xing, J., et al., Mitochondrial DNA content: its genetic heritability and association with renal cell carcinoma. Journal of the National Cancer Institute, 2008. 100(15): p. 1104-12.

159. Higuchi, M., et al., Mitochondrial DNA determines androgen dependence in prostate cancer cell lines. Oncogene, 2006. 25(10): p. 1437-45. 
160. Cheau-Feng Lin, F., et al., Mitochondrial DNA copy number is associated with diagnosis and prognosis of head and neck cancer. Biomarkers : biochemical indicators of exposure, response, and susceptibility to chemicals, 2014. 19(4): p. 269-74.

161. Lebedeva, M.A., J.S. Eaton, and G.S. Shadel, Loss of p53 causes mitochondrial DNA depletion and altered mitochondrial reactive oxygen species homeostasis. Biochimica et biophysica acta, 2009. 1787(5): p. 32834.

162. Singh, K.K., et al., Mutations in mitochondrial DNA polymerase-gamma promote breast tumorigenesis. J Hum Genet, 2009. 54(9): p. 516-24.

163. Chandra, D. and K.K. Singh, Genetic insights into OXPHOS defect and its role in cancer. Biochimica et biophysica acta, 2011. 1807(6): p. 620-5.

164. Woo, D.K., et al., Mitochondrial genome instability and ROS enhance intestinal tumorigenesis in APC(Min/+) mice. The American journal of pathology, 2012. 180(1): p. 24-31.

165. Guerra, F., et al., Mitochondrial DNA mutation in serous ovarian cancer: implications for mitochondriacoded genes in chemoresistance. Journal of clinical oncology : official journal of the American Society of Clinical Oncology, 2012. 30(36): p. e373-8.

166. Lebrecht, D., et al., Time-dependent and tissue-specific accumulation of mtDNA and respiratory chain defects in chronic doxorubicin cardiomyopathy. Circulation, 2003. 108(19): p. 2423-9.

167. Garrido, N., et al., Cisplatin-mediated impairment of mitochondrial DNA metabolism inversely correlates with glutathione levels. The Biochemical journal, 2008. 414(1): p. 93-102.

168. Olivero, O.A., et al., Preferential binding of cisplatin to mitochondrial DNA of Chinese hamster ovary cells. Mutation research, 1995. 346(4): p. 221-30.

169. Devarajan, P., et al., Cisplatin-induced apoptosis in auditory cells: role of death receptor and mitochondrial pathways. Hear Res, 2002. 174(1-2): p. 45-54.

170. Qian, W., et al., Mitochondrial density determines the cellular sensitivity to cisplatin-induced cell death. Am J Physiol Cell Physiol, 2005. 289(6): p. C1466-75.

171. Yang, Z., et al., Cisplatin preferentially binds mitochondrial DNA and voltage-dependent anion channel protein in the mitochondrial membrane of head and neck squamous cell carcinoma: possible role in apoptosis. Clin Cancer Res, 2006. 12(19): p. 5817-25.

172. Kong, Q., J.A. Beel, and K.O. Lillehei, A threshold concept for cancer therapy. Medical hypotheses, 2000. 55(1): p. 29-35.

173. Zhou, Y., et al., Free radical stress in chronic lymphocytic leukemia cells and its role in cellular sensitivity to ROS-generating anticancer agents. Blood, 2003. 101(10): p. 4098-104.

174. Penta, J.S., et al., Mitochondrial DNA in human malignancy. Mutation research, 2001. 488(2): p. 119-33.

175. Huang, P., et al., Superoxide dismutase as a target for the selective killing of cancer cells. Nature, 2000. 407(6802): p. 390-5.

176. Hileman, E.O., et al., Intrinsic oxidative stress in cancer cells: a biochemical basis for therapeutic selectivity. Cancer chemotherapy and pharmacology, 2004. 53(3): p. 209-19.

177. Joiner, M.C. and A.J. van der Kogel, eds. Basic clininal radiobiology. 4 ed. 2009, Hodder Arnold: London.

178. Bernstein, C., et al., DNA repair/pro-apoptotic dual-role proteins in five major DNA repair pathways: failsafe protection against carcinogenesis. Mutation research, 2002. 511(2): p. 145-78.

179. Ferrari, D., et al., Differential regulation and ATP requirement for caspase-8 and caspase-3 activation during CD95- and anticancer drug-induced apoptosis. J Exp Med, 1998. 188(5): p. 979-84.

180. Hopfner, K.P., et al., Structural biology of Rad50 ATPase: ATP-driven conformational control in DNA double-strand break repair and the ABC-ATPase superfamily. Cell, 2000. 101(7): p. 789-800.

181. Castedo, M., et al., Cell death by mitotic catastrophe: a molecular definition. Oncogene, 2004. 23(16): p. 2825-37.

182. Kroemer, G., L. Galluzzi, and C. Brenner, Mitochondrial membrane permeabilization in cell death. Physiological reviews, 2007. 87(1): p. 99-163.

183. Cloos, C.R., et al., Mitochondrial DNA depletion induces radioresistance by suppressing G2 checkpoint activation in human pancreatic cancer cells. Radiation research, 2009. 171(5): p. 581-7. 
184. Tang, J.T., et al., Mitochondrial DNA influences radiation sensitivity and induction of apoptosis in human fibroblasts. Anticancer research, 1999. 19(6B): p. 4959-64.

185. Heddle, J.A. and J.W. Harris, Letter: Rapid screening of radioprotective drugs in vivo. Radiation research, 1975. 61(2): p. 350-3.

186. Yamazaki, H., et al., Impact of mitochondrial DNA on hypoxic radiation sensitivity in human fibroblast cells and osteosarcoma cell lines. Oncology reports, 2008. 19(6): p. 1545-9.

187. Yoshioka, Y., et al., Impact of mitochondrial DNA on radiation sensitivity of transformed human fibroblast cells: clonogenic survival, micronucleus formation and cellular ATP level. Radiation research, 2004. 162(2): p. 143-7.

188. Kulkarni, R., et al., Mitochondrial gene expression changes in normal and mitochondrial mutant cells after exposure to ionizing radiation. Radiation research, 2010. 173(5): p. 635-44.

189. Kulkarni, R., R.A. Thomas, and J.D. Tucker, Expression of DNA repair and apoptosis genes in mitochondrial mutant and normal cells following exposure to ionizing radiation. Environmental and molecular mutagenesis, 2011. 52(3): p. 229-37.

190. Kulkarni, R., et al., Mitochondrial mutant cells are hypersensitive to ionizing radiation, phleomycin and mitomycin C. Mutation research, 2009. 663(1-2): p. 46-51.

191. Bol, V., et al., Reprogramming of tumor metabolism by targeting mitochondria improves tumor response to irradiation. Acta oncologica, 2015. 54(2): p.266-74

192. Wardell, T.M., et al., Changes in the human mitochondrial genome after treatment of malignant disease. Mutation research, 2003. 525(1-2): p. 19-27.

193. Jiang, W.W., et al., Decreased mitochondrial DNA content in posttreatment salivary rinses from head and neck cancer patients. Clinical cancer research : an official journal of the American Association for Cancer Research, 2006. 12(5): p. 1564-9.

194. Guo, Y., et al., The use of next generation sequencing technology to study the effect of radiation therapy on mitochondrial DNA mutation. Mutation research, 2012. 744(2): p. 154-60.

195. Owen, M.R., E. Doran, and A.P. Halestrap, Evidence that metformin exerts its anti-diabetic effects through inhibition of complex 1 of the mitochondrial respiratory chain. Biochem J, 2000. 348 Pt 3: p. 60714.

196. Lin, C.C., et al., Metformin enhances cisplatin cytotoxicity by suppressing signal transducer and activator of transcription-3 activity independently of the liver kinase B1-AMP-activated protein kinase pathway. American journal of respiratory cell and molecular biology, 2013. 49(2): p. 241-50.

197. Hou, X., et al., Metformin reduces intracellular reactive oxygen species levels by upregulating expression of the antioxidant thioredoxin via the AMPK-FOXO3 pathway. Biochemical and biophysical research communications, 2010. 396(2): p. 199-205.

198. Hu, F.B., et al., Prospective study of adult onset diabetes mellitus (type 2) and risk of colorectal cancer in women. Journal of the National Cancer Institute, 1999. 91(6): p. 542-7.

199. Evans, J.M., et al., Metformin and reduced risk of cancer in diabetic patients. BMJ, 2005. 330(7503): p. 1304-5.

200. Libby, G., et al., New users of metformin are at low risk of incident cancer: a cohort study among people with type 2 diabetes. Diabetes care, 2009. 32(9): p. 1620-5.

201. Li, B., et al., Inhibition of complex I regulates the mitochondrial permeability transition through a phosphate-sensitive inhibitory site masked by cyclophilin D. Biochimica et biophysica acta, 2012.

202. Shaw, R.J., et al., The LKB1 tumor suppressor negatively regulates mTOR signaling. Cancer cell, 2004. 6(1): p. 91-9.

203. Sanli, T., et al., lonizing radiation activates AMP-activated kinase (AMPK): a target for radiosensitization of human cancer cells. International journal of radiation oncology, biology, physics, 2010. 78(1): p. 221-9.

204. Song, C.W., et al., Metformin kills and radiosensitizes cancer cells and preferentially kills cancer stem cells. Sci Rep, 2012. 2: p. 362.

205. Storozhuk, Y., et al., Metformin inhibits growth and enhances radiation response of non-small cell lung cancer (NSCLC) through ATM and AMPK. British journal of cancer, 2013. 108(10): p. 2021-32. 
206. Cheong, J.H., et al., Dual inhibition of tumor energy pathway by 2-deoxyglucose and metformin is effective against a broad spectrum of preclinical cancer models. Molecular cancer therapeutics, 2011. 10(12): p. 2350-62.

207. Ben Sahra, I., et al., Targeting cancer cell metabolism: the combination of metformin and 2-deoxyglucose induces p53-dependent apoptosis in prostate cancer cells. Cancer research, 2010. 70(6): p. 2465-75.

208. Cao, X., et al., Glucose uptake inhibitor sensitizes cancer cells to daunorubicin and overcomes drug resistance in hypoxia. Cancer chemotherapy and pharmacology, 2007. 59(4): p. 495-505.

209. Liu, Y., et al., A small-molecule inhibitor of glucose transporter 1 downregulates glycolysis, induces cellcycle arrest, and inhibits cancer cell growth in vitro and in vivo. Molecular cancer therapeutics, 2012. 11(8): p. 1672-82.

210. Wood, T.E., et al., A novel inhibitor of glucose uptake sensitizes cells to FAS-induced cell death. Molecular cancer therapeutics, 2008. 7(11): p. 3546-55.

211. Pelicano, H., et al., Glycolysis inhibition for anticancer treatment. Oncogene, 2006. 25(34): p. 4633-46.

212. Stewart, J.D., et al., POLG mutations cause decreased mitochondrial DNA repopulation rates following induced depletion in human fibroblasts. Biochimica et biophysica acta, 2011. 1812(3): p. 321-5.

213. Heinen, E., et al., Effects of ethidium bromide on chick fibroblasts and mouse Ehrlich tumor cells cultivated in vitro. Cytological and cytochemical observations. Beitrage zur Pathologie, 1974. 153(4): p. 353-69.

214. van den Heuvel, L.P., J.A. Smeitink, and R.J. Rodenburg, Biochemical examination of fibroblasts in the diagnosis and research of oxidative phosphorylation (OXPHOS) defects. Mitochondrion, 2004. 4(5-6): p. 395-401.

215. Moran, M., et al., Mitochondrial bioenergetics and dynamics interplay in complex I-deficient fibroblasts. Biochimica et biophysica acta, 2010. 1802(5): p. 443-53.

216. Khan, S.M., R.M. Smigrodzki, and R.H. Swerdlow, Cell and animal models of mtDNA biology: progress and prospects. American journal of physiology. Cell physiology, 2007. 292(2): p. C658-69.

217. Trifunovic, A., et al., Premature ageing in mice expressing defective mitochondrial DNA polymerase. Nature, 2004. 429(6990): p. 417-23.

218. Ahlqvist, K.J., et al., Somatic progenitor cell vulnerability to mitochondrial DNA mutagenesis underlies progeroid phenotypes in Polg mutator mice. Cell metabolism, 2012. 15(1): p. 100-9.

219. Kruse, S.E., et al., Mice with mitochondrial complex I deficiency develop a fatal encephalomyopathy. Cell metabolism, 2008. 7(4): p. 312-20.

220. Valsecchi, F., et al., Metabolic consequences of NDUFS4 gene deletion in immortalized mouse embryonic fibroblasts. Biochimica et biophysica acta, 2012.

221. Ingraham, C.A., et al., NDUFS4: creation of a mouse model mimicking a Complex I disorder. Mitochondrion, 2009. 9(3): p. 204-10.

222. Camara, Y., et al., Administration of deoxyribonucleosides or inhibition of their catabolism as a pharmacological approach for mitochondrial DNA depletion syndrome. Human molecular genetics, 2014.

223. Gonzalez-Vioque, E., et al., Limited dCTP availability accounts for mitochondrial DNA depletion in mitochondrial neurogastrointestinal encephalomyopathy (MNGIE). PLoS genetics, 2011. 7(3): p. e1002035.

224. Haraguchi, M., et al., Targeted deletion of both thymidine phosphorylase and uridine phosphorylase and consequent disorders in mice. Molecular and cellular biology, 2002. 22(14): p. 5212-21.

225. Nathanson, D.A., et al., Co-targeting of convergent nucleotide biosynthetic pathways for leukemia eradication. The Journal of experimental medicine, 2014. 211(3): p. 473-86.

226. Akiyama, S., et al., The role of thymidine phosphorylase, an angiogenic enzyme, in tumor progression. Cancer science, 2004. 95(11): p. 851-7.

227. Koukourakis, M.I., et al., Different patterns of stromal and cancer cell thymidine phosphorylase reactivity in non-small-cell lung cancer: impact on tumour neoangiogenesis and survival. British journal of cancer, 1998. 77(10): p. 1696-703.

228. Moghaddam, A., et al., Thymidine phosphorylase is angiogenic and promotes tumor growth. Proceedings of the National Academy of Sciences of the United States of America, 1995. 92(4): p. 998-1002. 
229. Bronckaers, A., et al., The dual role of thymidine phosphorylase in cancer development and chemotherapy. Medicinal research reviews, 2009. 29(6): p. 903-53.

230. Hua, D., et al., Thymidylate synthase and thymidine phosphorylase gene expression as predictive parameters for the efficacy of 5-fluorouracil-based adjuvant chemotherapy for gastric cancer. World journal of gastroenterology: WJG, 2007. 13(37): p. 5030-4.

231. Guarcello, V., et al., Suppression of thymidine phosphorylase expression by promoter methylation in human cancer cells lacking enzyme activity. Cancer chemotherapy and pharmacology, 2008. 62(1): p. 8596.

232. Kosuri, K.V., et al., An epigenetic mechanism for capecitabine resistance in mesothelioma. Biochemical and biophysical research communications, 2010. 391(3): p. 1465-70.

233. Hecht, S.S., Cigarette smoking and lung cancer: chemical mechanisms and approaches to prevention. The lancet oncology, 2002. 3(8): p. 461-9.

234. Johnson, L., et al., Somatic activation of the K-ras oncogene causes early onset lung cancer in mice. Nature, 2001. 410(6832): p. 1111-6.

235. Artuso, L., et al., Mitochondrial DNA metabolism in early development of zebrafish (Danio rerio). Biochimica et biophysica acta, 2012. 1817(7): p. 1002-11.

236. Park, J., et al., Mitochondrial dysfunction in Drosophila PINK1 mutants is complemented by parkin. Nature, 2006. 441(7097): p. 1157-61.

237. Mori, C., T. Takanami, and A. Higashitani, Maintenance of mitochondrial DNA by the Caenorhabditis elegans ATR checkpoint protein ATL-1. Genetics, 2008. 180(1): p. 681-6.

238. Ezawa, K., et al., Evolutionary patterns of recently emerged animal duplogs. Genome biology and evolution, 2011. 3: p. 1119-35.

239. Liu, H., et al., Hypersensitization of tumor cells to glycolytic inhibitors. Biochemistry, 2001. 40(18): p. 5542-7.

240. Maher, J.C., A. Krishan, and T.J. Lampidis, Greater cell cycle inhibition and cytotoxicity induced by 2deoxy-D-glucose in tumor cells treated under hypoxic vs aerobic conditions. Cancer chemotherapy and pharmacology, 2004. 53(2): p. 116-22.

241. Mansi, J.L., et al., A phase I/ clinical and pharmacokinetic study of Lonidamine in patients with advanced breast cancer. British journal of cancer, 1991. 64(3): p. 593-7.

242. Lupescu, A., et al., Induction of apoptotic erythrocyte death by rotenone. Toxicology, 2012. 300(3): p. 132-7.

243. Lehman, A.J., The major toxic actions of insecticides. Bulletin of the New York Academy of Medicine, 1949. 25(6): p. 382-7.

244. Jalling, O. and C. Olsen, The effects of metformin compared to the effects of phenformin on the lactate production and the metabolism of isolated parenchymal rat liver cell. Acta pharmacologica et toxicologica, 1984. 54(5): p. 327-32.

245. Appleyard, M.V., et al., Phenformin as prophylaxis and therapy in breast cancer xenografts. British journal of cancer, 2012. 106(6): p. 1117-22.

246. Yuan, P., et al., Phenformin enhances the therapeutic benefit of BRAF(V600E) inhibition in melanoma. Proceedings of the National Academy of Sciences of the United States of America, 2013. 110(45): p. 18226-31

247. Jacobs, L.J., et al., Transmission and prenatal diagnosis of the T9176C mitochondrial DNA mutation. Molecular human reproduction, 2005. 11(3): p. 223-8.

248. van Den Bosch, B.J., et al., Mutation analysis of the entire mitochondrial genome using denaturing high performance liquid chromatography. Nucleic acids research, 2000. 28(20): p. E89.

249. White, H.E., et al., Accurate detection and quantitation of heteroplasmic mitochondrial point mutations by pyrosequencing. Genetic testing, 2005. 9(3): p. 190-9.

250. Fromenty, B., et al., Efficient and specific amplification of identified partial duplications of human mitochondrial DNA by long PCR. Biochimica et biophysica acta, 1996. 1308(3): p. 222-30.

251. Reynier, P. and Y. Malthiery, Accumulation of deletions in MtDNA during tissue aging: analysis by long PCR. Biochemical and biophysical research communications, 1995. 217(1): p. 59-67. 


\section{Chapter 2}

252. He, L., et al., Detection and quantification of mitochondrial DNA deletions in individual cells by real-time PCR. Nucleic acids research, 2002. 30(14): p. e68.

253. Jacobs, L.J., et al., Pearson syndrome and the role of deletion dimers and duplications in the mtDNA. Journal of inherited metabolic disease, 2004. 27(1): p. 47-55.

254. Vermulst, M., J.H. Bielas, and L.A. Loeb, Quantification of random mutations in the mitochondrial genome. Methods, 2008. 46(4): p. 263-8.

255. Taylor, S.D., et al., Targeted enrichment and high-resolution digital profiling of mitochondrial DNA deletions in human brain. Aging cell, 2014. 13(1): p. 29-38.

256. He, Y., et al., Heteroplasmic mitochondrial DNA mutations in normal and tumour cells. Nature, 2010. 464(7288): p. 610-4.

257. Maitra, A., et al., The Human MitoChip: a high-throughput sequencing microarray for mitochondrial mutation detection. Genome research, 2004. 14(5): p. 812-9.

258. van Eijsden, R.G., et al., Chip-based mtDNA mutation screening enables fast and reliable genetic diagnosis of OXPHOS patients. Genetics in medicine : official journal of the American College of Medical Genetics, 2006. 8(10): p. 620-7.

259. Taylor, R.W., et al., Selective inhibition of mutant human mitochondrial DNA replication in vitro by peptide nucleic acids. Nature genetics, 1997. 15(2): p. 212-5.

260. Oltersdorf, T., et al., An inhibitor of Bcl-2 family proteins induces regression of solid tumours. Nature, 2005. 435(7042): p. 677-81. 


\section{Chapter}

\section{Distinct radiation responses after in vitro mtDNA depletion are potentially related to oxidative stress}

Submitted

Marike W. van Gisbergen, An M. Voets, Rianne Biemans, Roland F. Hoffmann, MarieJosé Drittij-Reijnders, Guido R.M.M. Haenen, Irene H. Heijink, Kasper M.A. Rouschop, Ludwig Dubois*, Philippe Lambin*

* contributed equally 


\section{Abstract}

Several clinically used drugs are mitotoxic and cause mitochondrial DNA (mtDNA) depletion, mutations or deletions. Several studies have shown that these mtDNA alterations influence cancer treatment response. We hypothesized that radiation responsiveness will be enhanced in cellular models with decreased mtDNA content, attributed to altered ATP production, ROS production and antioxidant capacity. For this purpose, BEAS-2B (immortalized bronchial epithelium), A549 (lung adenocarcinomic epithelium) and 143B (osteosarcoma) cell lines were depleted from their mtDNA by ethidium bromide treatment generating $\rho^{0}$ cell lines. mtDNA depletion resulted in significantly $(p<0.05)$ decreased proliferation. Intrinsic radiosensitivity, assessed by the surviving fraction at 2Gy, was not influenced. However, at irradiation doses of 4Gy and higher, survival was increased $(p<0.001)$ for the BEAS-2B $\rho^{0}$ cells, while decreased for both tumor $\rho^{0}$ lines $(p<0.05)$. In agreement, increased residual DNA damage was observed after mtDNA depletion for A549 and 143B cells 24 hours after 4Gy irradiation. Since radiation therapy relies on the production of reactive oxygen species (ROS) for its lethal properties, we investigated whether ROS levels are causative for the alterated radiation response. Baseline ROS formation was similar between BEAS-2B parental and $\rho^{0}$ cells, while reduced in A549 and 143B $\rho^{0}$ cells, compared to their parental counterparts. ROS levels were significantly increased for all parental cell lines 24 hours after irradiation, while levels for the $\rho^{0}$ cells remained unchanged. Excessive production of ROS can result into oxidative stress if the antioxidant defense systems are insufficient or defective. The GSH:GSSG ratio can serve as indicator of such oxidative stress. Here we found that the GSH:GSSG ratios were in line with the altered radiation response. Exposure to irradiation reduced GSH:GSSG ratios for the BEAS-2B parental and $143 \mathrm{~B} \rho^{0}$, while for the A549 this ratio remained unchanged. Also, changed levels were observed for KEAP1 and for downstream targets of the Keap1-Nrf2 pathway. Our results indicate that mtDNA copy number depletion results in varying radiation responses depending on the background of the cell line, potentially involving variation in cellular ROS levels and antioxidant defense mechanisms. We therefore suggest that when mitotoxic drugs are combined with radiation, in particular at high dose per fraction, the effect of the mitotoxic drugs on mtDNA copy number should be explored. 


\section{Introduction}

Under normal physiological conditions, mitochondria are important organelles in the cell. One of their key functions is the production of adenosine triphosphate (ATP) via the oxidative phosphorylation system (OXPHOS), thereby providing the cell with its essential energy supply. It has been observed that tumor cells rely less on OXPHOS but are more dependent on aerobic glycolysis, also known as the Warburg effect [1]. New findings have revealed that this metabolic shift could be the result of metabolic reprogramming from a more OXPHOS to a more glycolytic phenotype (uncoupled glycolysis) regardless of oxygen presence in the tumor tissue, supporting the tumor's anabolic growth and microenvironmental adaptations. This may be a possible explanation for an increased metastatic potential of tumor cells [2, 3]. Potential causes for such metabolic reprogramming could be changes in the mitochondrial genome (mtDNA) such as deletions, substitutions and even mtDNA copy number variations, leading to dysfunctional mitochondria [4]. Mitochondria play an important role in processes like reactive oxygen production, redox signaling and apoptosis, while these processes influence significantly radiotherapy (RT) response [5-8]. Furthermore, repair of RT-induced damage is dependent on mitochondrial energy supply which heavily relies upon the functionality of mitochondria [9]. mtDNA alterations, such as mutations, deletions or copy number variations, may therefore influence RT response. These variations can be potentially caused by mitotoxic agents, such as cisplatin [10] or doxorubicin [11]. Cisplatin is able to induce a decrease in mtDNA copy number and impairment of mtRNA synthesis [10]. Doxorubicin intercalates with mtDNA and thereby contributes to mitochondrial toxicity [11]. Different types of alterations of the mtDNA genome have been found to be related to cancer and treatment outcome [4]. Cells fully depleted from their mitochondrial genome, Rho- $0\left(\rho^{0}\right)$ cells, are an extreme in vitro model to investigate the association between radiation outcome and dysfunctional mitochondria due to abnormalities in the mitochondrial genome [12, 13]. Several contradictory results on cellular mtDNA depletion using various (tumor) cell lines have been published. Different radiation responses were observed varying between a radioresistant phenotype to no difference in radiation response in vitro $[14,15]$. However, an increased radiation response was observed in an in vivo model [16]. To our knowledge no mechanistic insights have been proposed to explain the observed differences in radiation response of mtDNA depleted cell lines. Therefore, carefully elucidating the effect of these processes in radiation response will be important. We hypothesized that reduced mitochondrial function after mtDNA depletion changes the radiation response and this is dependent on altered ATP production, ROS production and on the cells' antioxidant capacity. 


\section{Material and Methods}

\section{Cell culture model}

The parental 143B and mtDNA depleted 143B Rho-0 $\left(\rho^{0}\right)$ osteosarcoma cells were cultured in Gibco's Dulbecco's modified Eagle's medium (DMEM, D-glucose $4.5 \mathrm{~g} / \mathrm{l}$ ) with $10 \%$ fetal bovine serum (FBS; Lonza), the latter supplemented with $150 \mu \mathrm{g} / \mathrm{ml}$ uridine and $100 \mu \mathrm{g} / \mathrm{ml}$ bromodeoxyuridine (Sigma-Aldrich) [17]. A549 (alveolar type-II carcinoma cells) $\rho^{0}$ cells were created by Prof. Dr. Ian Holt (Cambridge University, United Kingdom) and parental and $\rho^{0}$ cells were kindly provided by Dr. Lodovica Vergani (Padova University, Italy). mtDNA depletion of BEAS-2B (immortalized bronchial epithelial) cells was accomplished by culturing cells in medium supplemented with ethidium bromide $(50 \mathrm{ng} / \mathrm{ml}$; Sigma-Aldrich). Both A549 and BEAS-2B cells were cultured in DMEM (D-glucose $4.5 \mathrm{~g} / \mathrm{l}$ ) supplemented with 25\% FBS, vitamins, $1 \mathrm{X}$ essential and non-essential amino acids (Sigma-Aldrich) and $50 \mu \mathrm{g} / \mathrm{ml}$ uridine (Acros Organics).

\section{mtDNA copy number determination}

Confirmation of mtDNA depletion was obtained by performing quantitive PCR. DNA was isolated using the gentra puregene kit (Qiagen). Ratios of nDNA (B2M) and mtDNA (DLoop) were obtained in order to determine the mtDNA content. Primer secquences can be found in Supplementary Table 1. Quantitative PCR was performed on the 7900HT Fast Real-Time PCR System (Applied Biosystems). The PCR mixture contained 5ng/ul DNA, 0.3 uM forward and reverse primer and 1x master-mix (SensiMix SYBR ${ }^{\circledR}$ HiRox kit, Bioline Reagents). The cycling conditions were: $2^{\prime} 50^{\circ} \mathrm{C}, 10^{\prime} 95^{\circ} \mathrm{C}, 40$ cycles of $15^{\prime \prime}$ at $95^{\circ} \mathrm{C}+1^{\prime} 60^{\circ} \mathrm{C}$.

\section{Proliferation and clonogenic survival assay}

Proliferation was monitored during 7 days using the IncuCyte FLR after seeding 2500 cells/well. For clonogenic survival analysis, cells were seeded on day 0 and irradiated using a $225 \mathrm{kV}$ Philips X-ray tube on day 1 . Subsequently, cells were trypsinized and plated in triplicate for clonogenic survival. Cells were allowed to form colonies during 10 days, fixed and stained with a $0.4 \%$ methylene blue (Sigma-Aldrich) in $70 \%$ ethanol solution. Colonies were defined as $>50$ cells [18]. 


\section{Metabolic profiling}

Cells were seeded at an optimized cell density of $3 \times 10^{4}$ cells/well (BEAS-2B) or $1.5 \times 10^{4}$ cells/well (143B and A549). Metabolic profiles were generated by replacing the growth media by assay media 1 hour before using the Seahorse XF96 extracellular Flux analyzer (Seahorse Bioscience) according to manufacturer's guidelines [19]. A mitochondrial stress test was established measuring the oxygen consumption rate (OCR) after subsequent injections of $1 \mu \mathrm{M}$ oligomycin, optimized FCCP concentrations $0.3 \mu \mathrm{M}$ (A549), $0.5 \mu \mathrm{M}$ (143B) or $0.6 \mu \mathrm{M}$ (BEAS-2B) and $1 \mu \mathrm{M}$ mixture of rotenone and antimycin A (Sigma-Aldrich). The glycolysis stress test was performed by measuring extracellular acidification (ECAR) after sequential addition of $10 \mathrm{mM}$ glucose, optimized oligomycin concentration $1.0 \mu \mathrm{M}$ (all cell lines) and $0.1 \mathrm{M}$ 2-deoxyglucose (2-DG) (Sigma-Aldrich).

\section{Molecular assays}

ATP levels were measured based on the Cell-TiterGlo Luminescent cell viability test (Promega) on the Glomax 96 well luminometer (Promega). Levels of extracellular LLactic acid were measured as previously described [20]. Formation of reactive oxygen species (ROS) was detected 24 hours after ionizing radiation (4Gy). Cells were exposed for 1 hour to $20 \mu \mathrm{M}$ dihydrorhodamine (Invitrogen). Levels of ROS were determined in the propidium iodide (Sigma-Aldrich) negative population by flow cytometry (BD FACS Canto II) and expressed using mean fluorescent intensity. Glutathione levels (GSH and GSSG) were measured in the cell lysates as described previously [21].

\section{$\mathrm{Y}-\mathrm{H} 2 \mathrm{AX}$ immunocytochemistry}

Cells were fixed with $100 \%$ methanol at $-20^{\circ} \mathrm{C}$. Subsequently cells were permeabilized with $0.05 \%$ Tween-PBS and normal goat serum was used as blocking agent. Cells were stained with a primairy anti-phospho(Ser139)-H2AX antibody (1:500, Millipore) followed by anti-rabbit Alexa Fluor 488 (1:500, Invitrogen) as secundairy antibody. Hoechst33342 (20 ug/ml, Sigma-Aldrich) was used as nuclear counter stain. Images were acquired using a Leica TCS SPE confocal microscope.

\section{RNA isolation and quantitative PCR analysis}

mRNA was extracted using the NucleoSpin RNA II kit (Bioke) using iScript cDNA Synthesis Kit (BioRad). Both methods were performed according to the manufacturers' instructions. Quantitative PCR was performed on the CFX96 (Biorad). The abundance of the genes of interest were detected with $\mathrm{SYBR}^{\circledR}$ Green I (Eurogentec). Values for each 
gene were normalized to $18 \mathrm{~S}$ expression levels. Primer sequences are listed in Supplementary Table 1.

\section{Statistics}

All statistical analyses were performed with GraphPad Prism version 5.03 for Windows (GraphPad Software, 2009, California, USA). A non-parametric Mann-Whitney U test for small groups was used to determine the statistical significance of differences between two independent groups of variables. Clonogenic survival curves were compared using an extra sum F-test. For all tests, a $p<0.05$ was considered significant.

\section{Results}

To investigate if extreme changes in OXPHOS capacity affect radiosensitivity, we used $\rho^{0}$ cell lines with different genetic backgrounds. All our $\rho^{0}$ cell lines showed a strong and significant decrease in mtDNA copy number (Fig. 1A; BEAS-2B $p<0.001$; A549 $p<0.05$; 143B $\mathrm{p}<0.05)$. Additionally, proliferation was decreased as evaluated by an increased doubling time (on average 54\%) for all $\rho^{0}$ cell lines (Fig. 1A). We confirmed the drastic effect on OXPHOS upon mtDNA depletion as basal respiration was almost absent $(p<0.05)$ in the BEAS-2B, A549 and 143B $\rho^{0}$ (Fig. 1B) while the parental cells had a functional OXPHOS system with spare capacity (Fig. 1C). To compensate their loss of OXPHOS function, $\rho^{0}$ cells showed an enhanced $(p<0.05)$ glucose metabolism accompanied with a loss in glycolytic reserve (Fig. 1C; Fig S1). The mtDNA depletion did not result in reduced cellular ATP levels and no differences in lactic acid production were observed between $\rho^{0}$ and their parental counterparts (Fig. 1D). Together our results indicate that mtDNA depleted cells upscaled their glucose metabolism. 
A
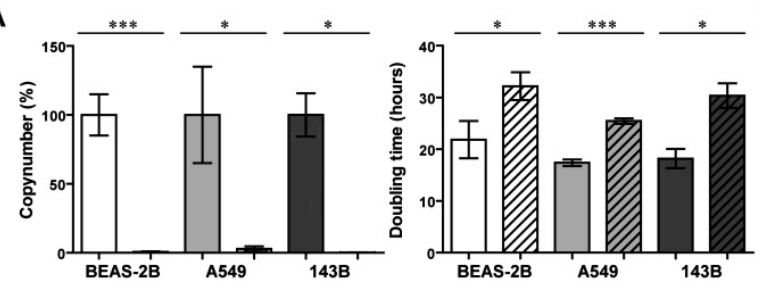

Mito stress test

C

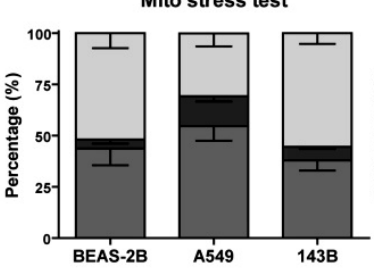

Glycolysis stress test

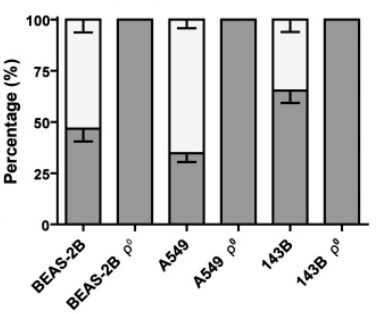

B

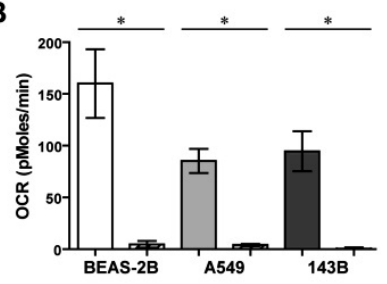

\section{D}
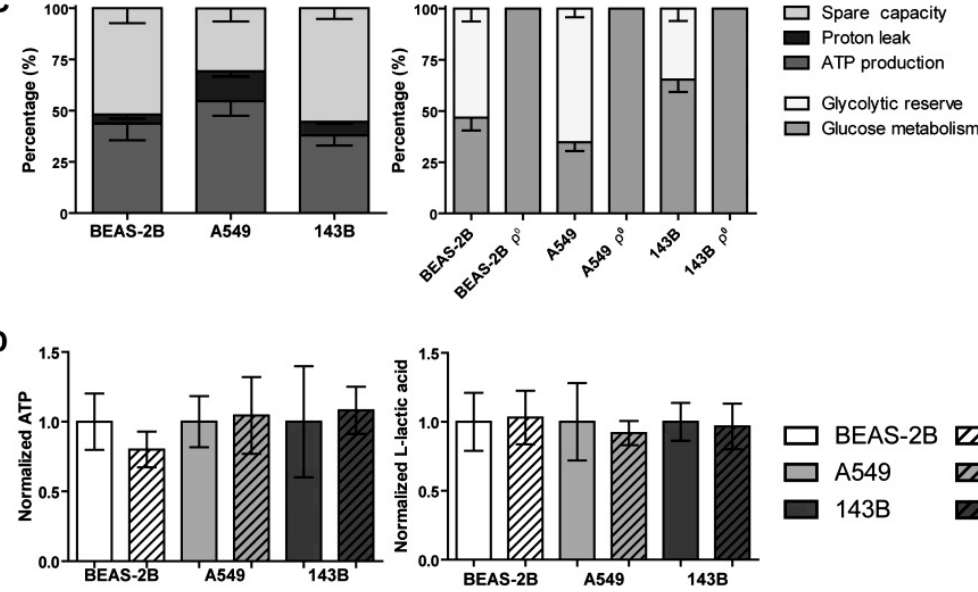

Figure 1. Validation of mtDNA depleted cell lines

A. qPCR assessed mtDNA copy number levels expressed in percentage normalized to each parental (left panel) and the doubling time for all investigated cell lines (right panel). B. Basal respiration expressed as oxygen consumption rate (OCR) in function of time (minutes). C. Stacked plot of mitochondrial (left panel) or glycolysis (right panel) stress test after measuring OCR or extracellular acidification rate (ECAR) respectively. D. ATP levels (left panel) and L-Lactic acid levels (right panel), both normalized to each parental. Data represent the mean \pm SEM from at least 3 independent biological experiments. ${ }^{*} p<0.05,{ }^{* * *} p<0.001$.

In order to assess the influence of mtDNA depletion on radiation response, clonogenic survival assays were performed. Overall survival was increased $(p<0.001)$ for the BEAS2B $\rho^{0}$ cells, while decreased for A549 $\rho^{0}(p<0.01)$ and 143B $\rho^{0}(p<0.0001)$ cells (Fig. $\left.2 A\right)$. However, the intrinsic radiosensitivity assessed by the surviving fraction at $2 \mathrm{~Gy}$ was not influenced ( $p>0.05$ ). mtDNA depleted cells showed differences in residual DNA damage 24 hours after 4Gy irradiation as compared to the parental cell lines in line with the clonogenic survival data. An increased number of residual $\mathrm{pH} 2 \mathrm{AX}$ foci was observed for the 143B $\rho^{0}$ and to a lesser extent for the A549 $\rho^{0}$ cells. The opposite was observed for the BEAS-2B, where the $\rho^{0}$ cells showed a lower residual signal compared to the parental cell line (Fig. 2B). 
A

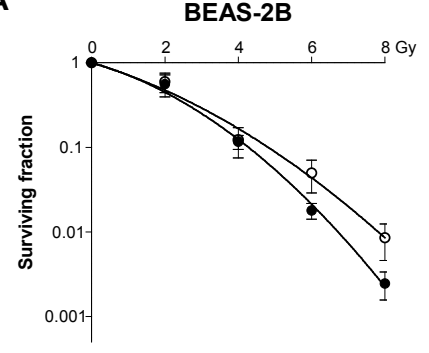

A549

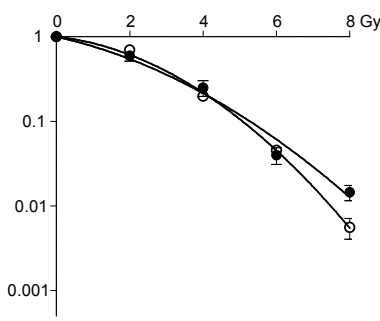

143B

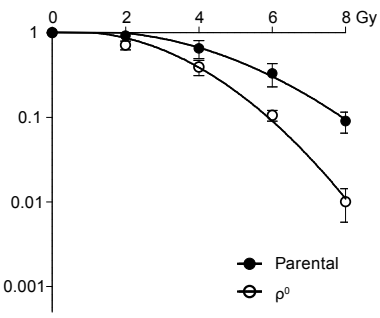

B

Parental

4Gy 24H

OGy

$\rho^{0}$

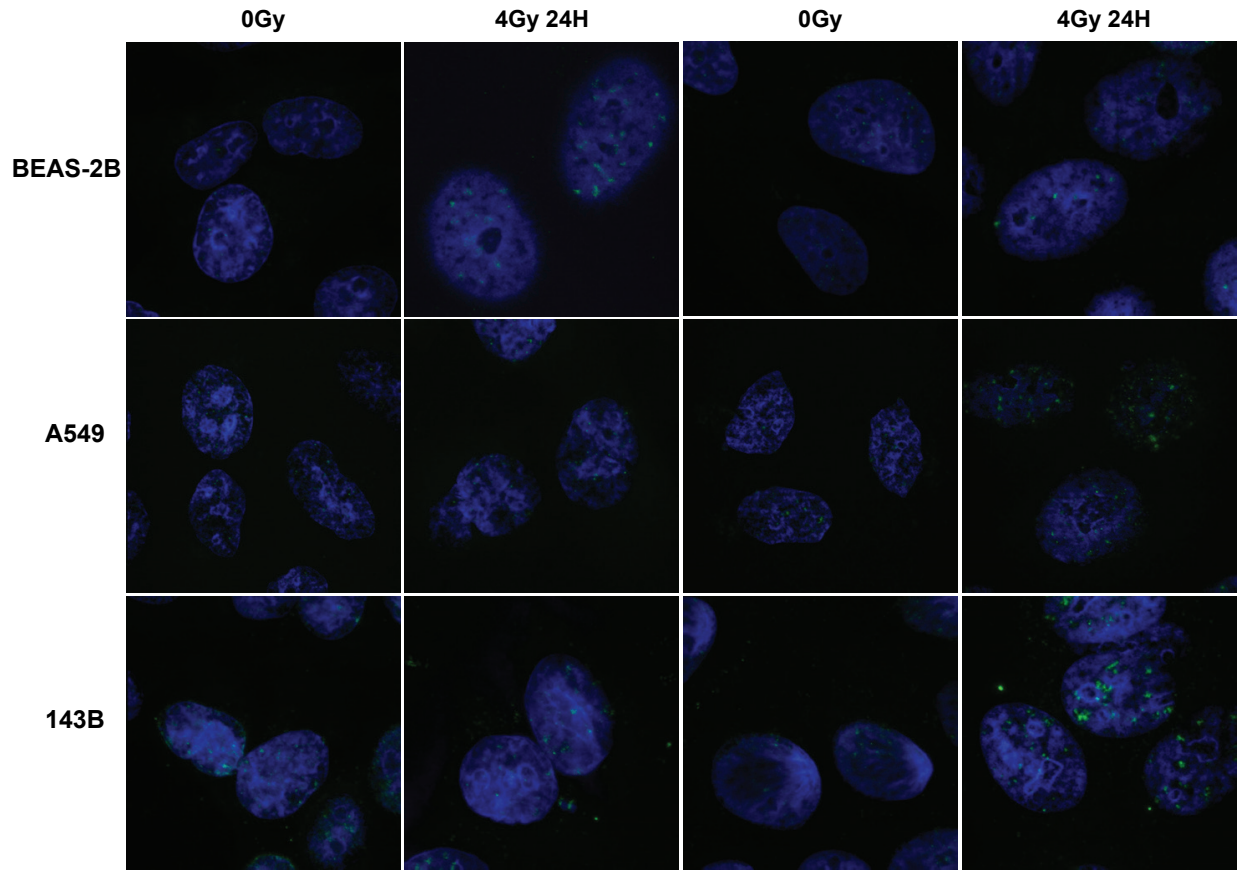

Figure 2. Radiation response in mtDNA depleted cell lines

A. Clonogenic survival plots, fitted according to the LQ model. Results show mean \pm SEM from at least 3 independent biological replicates. 0 Gy conditions are sham irradiated. B. Representative merged fluorescent images of $\mathrm{YH} 2 \mathrm{AX}$ foci (green) and nuclei (blue) visualizing residual foci upon irradiation.

Radiation therapy relies on the production of reactive oxygen species (ROS) for its lethal properties. Additionally, cellular mitochondrial function plays an important role in ROS production and redox signaling. Here we assessed ROS formation (Fig. $3 \mathrm{~A}$ ) at baseline (OGy), which was found to be similar $(p=0.878)$ for BEAS-2B parental and $\rho^{0}$ cell lines, while reduced for A549 (NS) and 143B ( $p=0.0211) \rho^{0}$ cells. ROS levels were significantly increased $(p<0.05)$ for all parental cell lines 24 hours after irradiation, while levels for the $\rho^{0}$ cell lines remained equal. ROS formation 24 hours after irradiation was significantly 
lower $(p<0.05)$ for the the A459 and 143B $\rho^{0}$ cell lines compared to their parentals. Since "oxidative stress" indicates a disbalance between ROS and antioxidants in favor for ROS, we investigated the antioxidant capacity of the $\rho^{0}$ cell lines using the ratio of GSH:GSSG as a proxy to assess if ROS scavenging mechanisms were altered. GSH:GSSG ratios were decreased for the BEAS-2B parental and 143B $\rho^{0}$ cells upon irradiation. No alterations were observed for both $\mathrm{A} 549$ cell lines (Fig. 3B). The kelch-like ECH-associated protein 1 (Keap1) - Nuclear factor erythroid 2 (NF-E2)-related factor 2 (Nrf2) pathway is an important oxidative stress response regulator and down-stream targets of Nrf2 are found to be involved in NADPH production, glutathione metabolism and utilization [22]. KEAP1 mRNA levels were elevated for both tumor $\rho^{0}$ cell lines at baseline and after irradiation compared to their parentals (Fig. 3C). mRNA expression levels of NRF2 were not significantly altered. Certain downstream targets of the Keap1-Nrf2 pathway such as the malic enzyme 1 (ME1), involved in NADPH production, displayed elevated mRNA levels in the tumor $\rho^{0}$ cell lines (Fig. S2) while other targets such as glucose-6-phosphate dehydrogenase (G6PD), phosphogluconate dehydrogenase (PGD), NAD(P)H quinone dehydrogenase 1 (NQO1, involved in quinone detoxification) and glutathione peroxidase (GPX1) did not show altered mRNA expression levels for the $\rho^{0}$ cells (Fig. S2). Baseline levels of the antioxidant superoxide dismutase 2 (SOD2) were elevated in the $\rho^{0}$ cells (Fig. $3 C)$, being significant for A549 ( $p=0.0493)$. Irradiation increased SOD2 expression for both parental A549 $(p<0.05)$ and $\rho^{0}(\mathrm{NS} ; \mathrm{p}=0.0519)$ cells. On the other hand, SOD2 expression levels were decreased upon irradiation for $143 \mathrm{~B}$ parental cells $(p=0.0093)$. Irradiation did not alter SOD2 expression levels for the 143B $\rho^{0}$ cells $(p=0.5476)$ neither for both BEAS-2B cell lines. Expression levels of SOD1 were not significantly altered upon irradiation for the BEAS-2B, A549 and 143B cells (Fig. S2). 
A
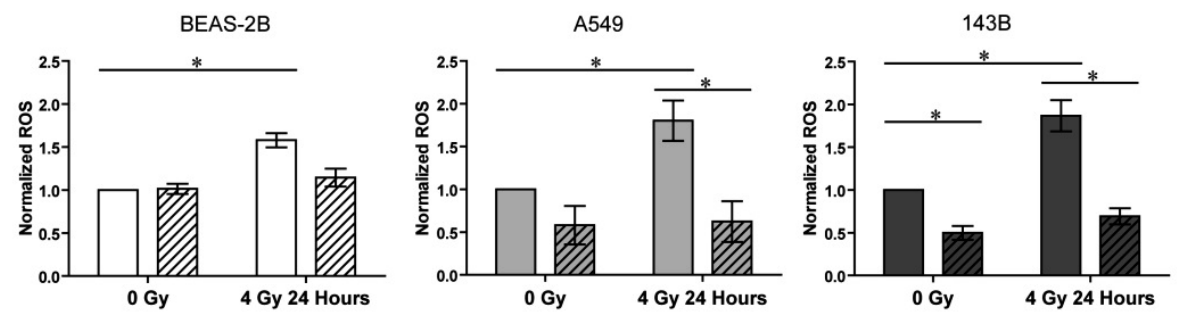

B

BEAS-2B

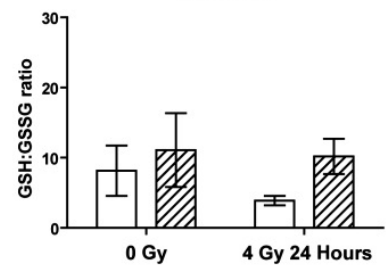

A549
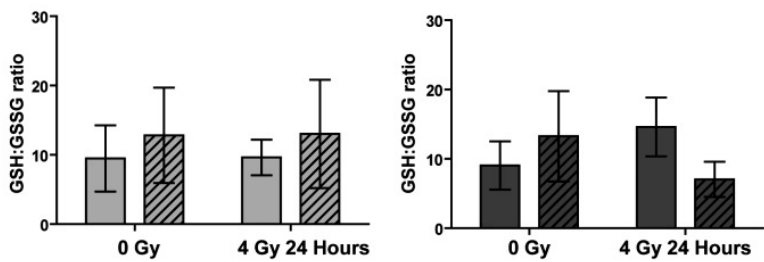

C
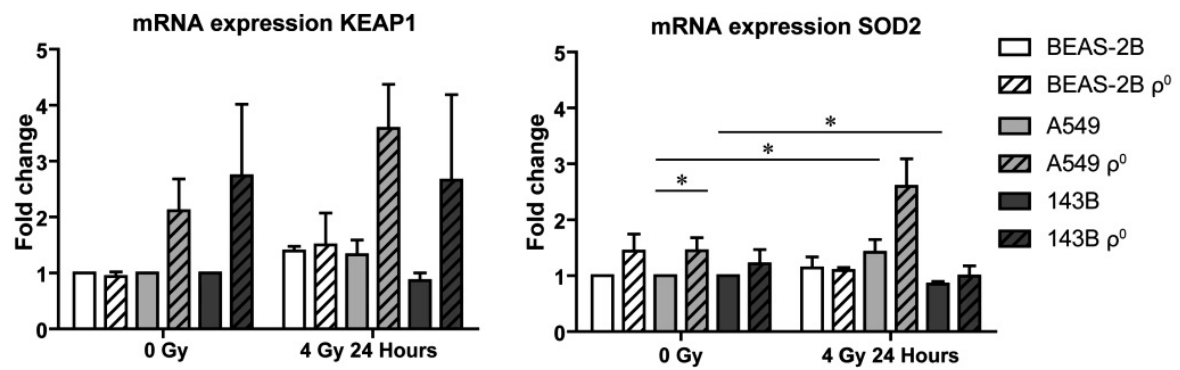

Figure 3. ROS and GSH levels at basal levels and 24 hours after 4Gy of irradiation

A. ROS levels at baseline and 24 hours after irradiation, normalized to each parental at baseline. B. Ratio of GSH:GSSG levels at baseline and at 24 hours after irradiation for parental and $\rho^{0}$ cell lines. Data represents the mean \pm SEM from at least 3 independent biological repeats. mtDNA depleted cells are indicated by the dashed bars. ${ }^{*} p<0.05$. C. mRNA expression of KEAP1 and SOD2 24 hours after irradiation, normalized to each parental at baseline. Data represents the mean + SEM from at least 2 independent biological repeats. * $p<0.05$.

\section{Discussion}

Recently, mtDNA variations have been linked to a wide variety of cancers and cancer outcome through influencing mitochondrial pathways [4] and may explain the hampered cellular bioenergetics found in many cancer types. Mitochondrial dysfunction has been found to be related to a chemoresistant phenotype in vitro [23-26] and chemotherapeutic agents might be associated with a decreased mitochondrial function $[10,11,27]$. Furthermore, several studies investigated the effect of mtDNA depletion with respect to radiotherapy by using different (tumor) cell lines with contradictory 
results, from a radioresistant phenotype or no difference in radiation response in vitro $[14,15,28]$ to an increased radiation response in vivo [16].

Reactive oxygen species (ROS) are formed in the presence of oxygen and are further induced upon radiation resulting in DNA damage. Functional mitochondria are found to be essential for radiation-induced ROS production [29]. Radiation causes either cell death (mainly by mitotic catastrophy) or induces a sublethal DNA damage initiating a temporary cell cycle arrest in order to repair the damage [4]. The interplay between mitochondrial function and radiation response is of great importance in such a radiation-induced DNA damage repair [30]. As both ROS production and ATP are vital parameters for radiotherapy outcome and mitochondrial function is essential for radiation-induced DNA repair, mtDNA variations could alter the response to irradiation possibly via increased lactate production as a consequence of a reduced OXPHOS function [25, 31]. Additionally, it has been shown that Increased lactate results in a radioresistant phenotype [32]. However, in our study, no differences could be observed in cellular ATP levels for mtDNA depleted cells, in agreement with the observed compensation by glycolysis induction often found in cell lines with a dysfunctional OXPHOS system [33, 34]. Although mtDNA depletion resulted in a glycolytic phenotype, no elevated lactic acid production was observed. A possible explanation for this finding could be that most lactic acid is catabolized in order to control the intracellular $\mathrm{pH}$ of the cell $[35,36]$.

We hypothesized that mtDNA depletion results in a more radiosensitive phenotype related to altered ROS and ATP levels and changes in the antioxidant ROS scavenging capacity. Our data showed, in line with literature, that a varying irradiation response occurred in different cell lines after mtDNA depletion. Overall, in our study the immortalized epithelial BEAS-2B $\rho^{0}$ cell line was less radiosensitive compared to the parental line, while the tumor derived $\rho^{0}$ cell lines (A549 and 143B) were more prone to a decreased survival upon radiation. However, the intrinsic radiosensitivity, correlated to clinical outcome after conventional fractionated radiotherapy and quantified by the survival fraction at 2Gy, was not significantly modified [37]. Residual $\gamma \mathrm{H} 2 \mathrm{AX}$ foci after irradiation were in agreement with the survival data, as described previously [38]. Irradiation is able to elevate ROS levels [7]. In the parental cell lines used in this study, the irradiation-induced increase in ROS levels was evident, while no increase was observed in $\rho^{0}$ cells. Potentially, this is due to the fact that both NADH ubiquinone oxidoreductase $(\mathrm{Cl})$ and ubiquinol-cytochrome c oxidoreductase (CIII), the major ROS generating sites of the oxidative phosphorylation chain, might be deficient in the established $\rho^{0}$ cells. This may explain why we found relatively lower ROS levels in the $\rho^{0}$ tumor cell lines [39-41]. In contrast to the elevated ROS levels found in cells with mtDNA variations $[25,42,43]$, Park et al. demonstrated that $\rho^{0}$ cells can also have normal ROS levels due to the protective up-regulation of their antioxidant system during the $\rho^{0}$ development [25]. 
Glutathione and SOD2 are important antioxidants that can be located in the mitochondrion [44]. Here we observed that the ratio between GSH and GSSG was decreased upon irradiation for the parental BEAS-2B and the $143 \mathrm{~B} \rho^{0}$ cells, whereas no differences were found for the A549 cells. These data suggest that differences in radiation response between parental and mtDNA depleted cells could be determined by the antioxidant capacity of the cells. SOD2 is an antioxidant able to scavenge superoxide radicals in the mitochondrial matrix [45] and therefore can protect cells against radiation [46]. Cells with an overexpression of SOD2 are found to have a higher cell survival [46]. We indeed observed an increase in SOD2 levels upon irradiation for the A549 parental cells. On the other hand, mitochondrial SOD2 levels where unaffected in the 143B cells. Previously, it has been reported that $\rho^{0}$ cells originating from different tissues can have altered glutathione antioxidant mechanisms [47, 48]. The Keap1-Nrf2 pathway is an important oxidative stress response regulator and downstream targets of Nrf2 are found to be involved in glutathione metabolism and utilization [22, 49]. KEAP1 mRNA levels were found to be elevated for both tumor mtDNA depleted lines, suggesting a role for this pathway in the antioxidant response. Some, but not all, downstream targets of the Keap1-Nrf2 pathway were found to be elevated indicating that Keap1-Nrf2 could play a role in the antioxidant capacity of $\rho^{0}$, however this remains to be further elucidated. Furthermore, it has also been suggested that variations in culture medium can influence oxidative stress and oxidative stress responses, possibly explaining the observed differences in antioxidant mechanisms $[48,50]$. A well-known example is cysteine, the precursor for glutathione [50]. Therefore, the use of glutathione and its effectors in culture medium should be carefully considered when elucidating the interactions of mitochondrial function and antioxidants.

Taken together, mtDNA depletion resulted in decreased proliferation for all $\rho^{0}$ cell lines, increased clonogenic survival for the epithelial BEAS-2B cells, but reduced clonogenic survival at higher irradiation doses for the tumor cell lines. Our findings indicate that inhibition of OXPHOS might be useful to enhance radiotherapy effects, in particular for patients treated with hypofractionated radiotherapy in rapidly proliferating tumors. This is in line with the well-known OXPHOS inhibitor metformin, an FDA approved drug for diabetes leading to an improvement of tumor oxygenation and better outcome following radiotherapy $[51,52]$. Concluding, mtDNA depletion resulted in varying irradiation responses in different cell lines, potentially attributed to ROS and antioxidant capacity.

\section{Conflict of interest statement}

The authors have no conflict to declare. 


\section{Acknowledgements}

This research was financially supported the Netherlands Genomics Initiative (pre-seed grant 2012, 93612005). 


\section{Supplementary Data}

\section{BEAS-2B}

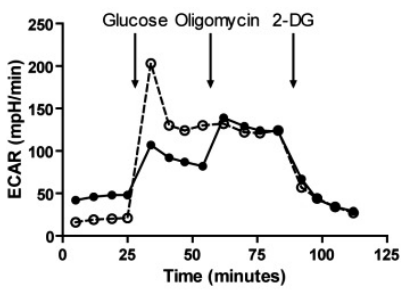

- Parental
A549

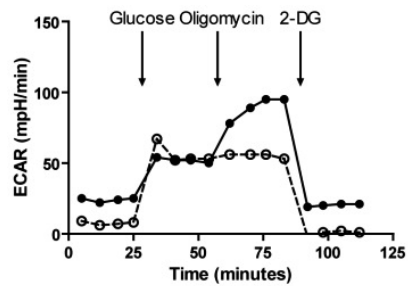

143B

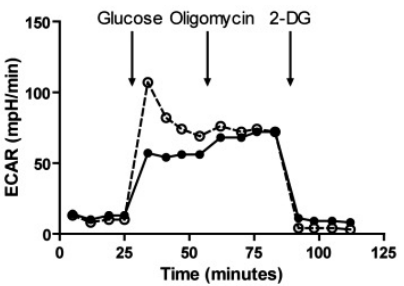

Supplementary Figure S1. Representative images of the glycolysis stress test for BEAS-2B, A549 and 143B.

Basal measurements followed by subsequent injections of $10 \mathrm{mM}$ glucose, $1.0 \mu \mathrm{M}$ oligomycin and $0.1 \mathrm{M} \mathrm{2-}$ deoxyglucose. 

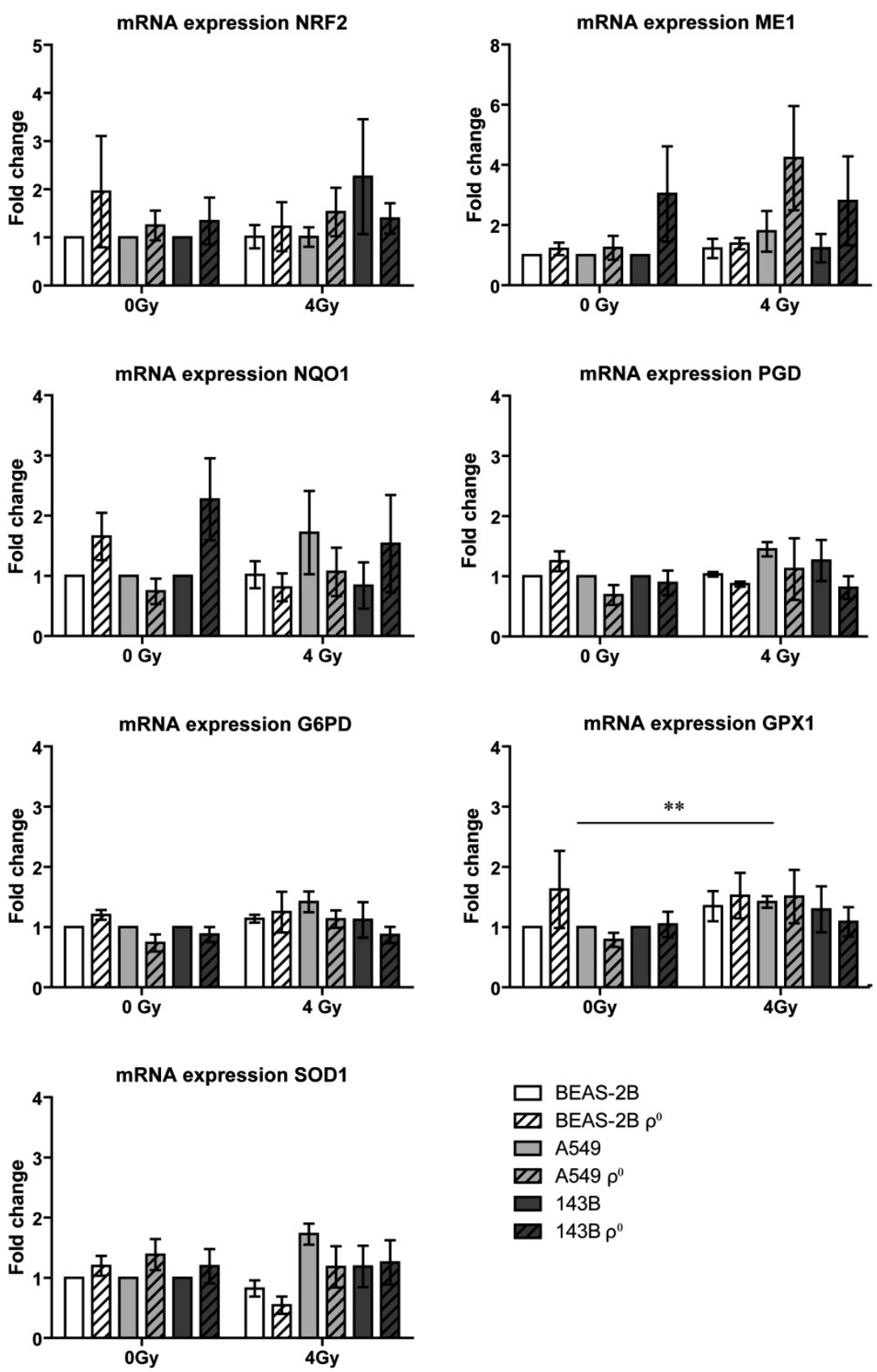

Supplementary Figure S2. Expression of antioxidant genes.

Data represents the mean \pm SEM from at least 2 independent biological repeats, normalized to each parental at baseline $* * p<0.01$. 
Chapter 3

Table S1. Quantitative PCR primer sequences

\begin{tabular}{lll}
\hline Target gene & Forward primer & Reverse primer \\
\hline D-loop & CATCTGGTTCCTACTTCAGGG & TGAGTGGTTAATAGGGTGATAGA \\
B2M & TGCTGTCTCCATGTTTGATGTATCT & TCTCTGCTCCCCACCTCTAAGT \\
18S & AGTCCCTGCCCTTTGTACACA & GATCCGAGGGCTCACTAAAC \\
KEAP1 & AACGGTGCTGTCATGTACCA & CCAATCTGCTCAGCGAAGTT \\
NRF2 & GAATACTCCCTGCAGCAAACAA & CTGGCTTCTTACTTTTGGGAACA \\
ME1 & GGCTGGTTGATTCAAAAGGA & TCTTCATGTTCATCGGCAAA \\
NOO1 & GCACTGATCGTACTGGCTCA & CATGGCATAGAGGTCCGACT \\
PGD & TGAGGCATACCACCTGATGA & CCATCGGTGTTTGGAACTT \\
G6PD & CCAAGCCCATCCCCTATATT & CCACTTGTAGGTGCCCTCAT \\
SOD1 (CuZnSOD) & GCGGAGGTCTGGCCTATAAAG & CTGGTTCCGAGGACTGCAA \\
SOD2 (MnSOD) & GCTCCGGTTTTGGGGTATCTG & GCGTTGATGTGAGGTTCCAG \\
GPX1 & TATCGAGAATGTGGCGTCCC & TCTTGGCGTTCTCCTGATGC \\
\hline
\end{tabular}




\section{References}

1. Warburg, O., F. Wind, and E. Negelein, The Metabolism of Tumors in the Body. The Journal of general physiology, 1927. 8(6): p. 519-30.

2. Hanahan, D. and R.A. Weinberg, Hallmarks of cancer: the next generation. Cell, 2011. 144(5): p. 646-74.

3. Porporato, P.E., et al., A mitochondrial switch promotes tumor metastasis. Cell reports, 2014. 8(3): p. 754-66.

4. van Gisbergen, M.W., et al., How do changes in the mtDNA and mitochondrial dysfunction influence cancer and cancer therapy? Challenges, opportunities and models. Mutation research. Reviews in mutation research, 2015. 764: p. 16-30.

5. Bernstein, C., et al., DNA repair/pro-apoptotic dual-role proteins in five major DNA repair pathways: failsafe protection against carcinogenesis. Mutation research, 2002. 511(2): p. 145-78.

6. Castedo, M., et al., Cell death by mitotic catastrophe: a molecular definition. Oncogene, 2004. 23(16): p. 2825-2837.

7. Lee, S., et al., An effective strategy for increasing the radiosensitivity of Human lung Cancer cells by blocking Nrf2-dependent antioxidant responses. Free radical biology \& medicine, 2012. 53(4): p. 807-16.

8. Cadenas, E. and K.J. Davies, Mitochondrial free radical generation, oxidative stress, and aging. Free radical biology \& medicine, 2000. 29(3-4): p. 222-30.

9. Liu, J., et al., Enhanced cytotoxic effect of low doses of metformin combined with ionizing radiation on hepatoma cells via ATP deprivation and inhibition of DNA repair. Oncology reports, 2012. 28(4): p. 1406-12.

10. Garrido, N., et al., Cisplatin-mediated impairment of mitochondrial DNA metabolism inversely correlates with glutathione levels. The Biochemical journal, 2008. 414(1): p. 93-102.

11. Ashley, N. and J. Poulton, Mitochondrial DNA is a direct target of anti-cancer anthracycline drugs. Biochemical and biophysical research communications, 2009. 378(3): p. 450-5.

12. Chandel, N.S. and P.T. Schumacker, Cells depleted of mitochondrial DNA (rhoO) yield insight into physiological mechanisms. FEBS letters, 1999. 454(3): p. 173-6.

13. King, M.P. and G. Attardi, Human cells lacking mtDNA: repopulation with exogenous mitochondria by complementation. Science, 1989. 246(4929): p. 500-3.

14. Cloos, C.R., et al., Mitochondrial DNA depletion induces radioresistance by suppressing G2 checkpoint activation in human pancreatic cancer cells. Radiation research, 2009. 171(5): p. 581-7.

15. Tang, J.T., et al., Mitochondrial DNA influences radiation sensitivity and induction of apoptosis in human fibroblasts. Anticancer research, 1999. 19(6B): p. 4959-64.

16. Bol, V., et al., Reprogramming of tumor metabolism by targeting mitochondria improves tumor response to irradiation. Acta oncologica, 2015. 54(2): p. 266-74.

17. Mineri, R., et al., How do human cells react to the absence of mitochondrial DNA? PloS one, 2009. 4(5): p. e5713.

18. Dubois, L., et al., Targeting carbonic anhydrase IX by nitroimidazole based sulfamides enhances the therapeutic effect of tumor irradiation: a new concept of dual targeting drugs. Radiotherapy and oncology : journal of the European Society for Therapeutic Radiology and Oncology, 2013. 108(3): p. 523-8.

19. Nicholls, D.G., et al., Bioenergetic profile experiment using C2C12 myoblast cells. Journal of visualized experiments : JoVE, 2010(46).

20. Dubois, L., et al., Specific inhibition of carbonic anhydrase IX activity enhances the in vivo therapeutic effect of tumor irradiation. Radiotherapy and oncology : journal of the European Society for Therapeutic Radiology and Oncology, 2011. 99(3): p. 424-31.

21. Rahman, I., A. Kode, and S.K. Biswas, Assay for quantitative determination of glutathione and glutathione disulfide levels using enzymatic recycling method. Nature protocols, 2006. 1(6): p. 3159-65.

22. Gorrini, C., I.S. Harris, and T.W. Mak, Modulation of oxidative stress as an anticancer strategy. Nature reviews. Drug discovery, 2013. 12(12): p. 931-47. 


\section{Chapter 3}

23. Kuwahara, Y., et al., The Involvement of Mitochondrial Membrane Potential in Cross-Resistance Between Radiation and Docetaxel. International journal of radiation oncology, biology, physics, 2016. 96(3): p. 556-65.

24. Singh, K.K., et al., Mitochondrial DNA determines the cellular response to cancer therapeutic agents. Oncogene, 1999. 18(48): p. 6641-6.

25. Park, S.Y., et al., Resistance of mitochondrial DNA-depleted cells against cell death: role of mitochondrial superoxide dismutase. The Journal of biological chemistry, 2004. 279(9): p. 7512-20.

26. Li, X., et al., MtDNA depleted PC3 cells exhibit Warburg effect and cancer stem cell features. Oncotarget, 2016. 7(26): p. 40297-40313.

27. Robertson, J.D., et al., Distinct pathways for stimulation of cytochrome c release by etoposide. The Journal of biological chemistry, 2000. 275(42): p. 32438-43.

28. $\mathrm{Xu}, \mathrm{H}$., et al., Prognostic value of mitochondrial DNA content and G10398A polymorphism in non-small cell lung cancer. Oncology reports, 2013. 30(6): p. 3006-12.

29. Leach, J.K., et al., lonizing radiation-induced, mitochondria-dependent generation of reactive oxygen/nitrogen. Cancer research, 2001. 61(10): p. 3894-901.

30. Kulkarni, R., R.A. Thomas, and J.D. Tucker, Expression of DNA repair and apoptosis genes in mitochondrial mutant and normal cells following exposure to ionizing radiation. Environmental and molecular mutagenesis, 2011. 52(3): p. 229-37.

31. Birsoy, K., et al., Metabolic determinants of cancer cell sensitivity to glucose limitation and biguanides. Nature, 2014. 508(7494): p. 108-12.

32. Quennet, V., et al., Tumor lactate content predicts for response to fractionated irradiation of human squamous cell carcinomas in nude mice. Radiotherapy and oncology : journal of the European Society for Therapeutic Radiology and Oncology, 2006. 81(2): p. 130-5.

33. Moran, M., et al., Mitochondrial bioenergetics and dynamics interplay in complex I-deficient fibroblasts. Biochimica et biophysica acta, 2010. 1802(5): p. 443-53.

34. Marin, J.J., E. Lozano, and M.J. Perez, Lack of mitochondrial DNA impairs chemical hypoxia-induced autophagy in liver tumor cells through ROS-AMPK-ULK1 signaling dysregulation independently of HIF1alpha. Free radical biology \& medicine, 2016. 101: p. 71-84.

35. Parks, S.K., J. Chiche, and J. Pouyssegur, Disrupting proton dynamics and energy metabolism for cancer therapy. Nature reviews. Cancer, 2013. 13(9): p. 611-23.

36. Kennedy, K.M., et al., Catabolism of exogenous lactate reveals it as a legitimate metabolic substrate in breast cancer. PloS one, 2013. 8(9): p. e75154.

37. Fertil, B. and E.P. Malaise, Intrinsic radiosensitivity of human cell lines is correlated with radioresponsiveness of human tumors: analysis of 101 published survival curves. International journal of radiation oncology, biology, physics, 1985. 11(9): p. 1699-707.

38. Menegakis, A., et al., Prediction of clonogenic cell survival curves based on the number of residual DNA double strand breaks measured by gammaH2AX staining. International journal of radiation biology, 2009. 85(11): p. 1032-41.

39. Chen, Q., et al., Production of reactive oxygen species by mitochondria: central role of complex III. The Journal of biological chemistry, 2003. 278(38): p. 36027-31.

40. Sugioka, K., et al., Mechanism of O2-generation in reduction and oxidation cycle of ubiquinones in a model of mitochondrial electron transport systems. Biochimica et biophysica acta, 1988. 936(3): p. 377-85.

41. Turrens, J.F. and A. Boveris, Generation of superoxide anion by the NADH dehydrogenase of bovine heart mitochondria. The Biochemical journal, 1980. 191(2): p. 421-7.

42. Petros, J.A., et al., mtDNA mutations increase tumorigenicity in prostate cancer. Proc Natl Acad Sci U S A, 2005. 102(3): p. 719-24.

43. Voets, A.M., et al., Patient-derived fibroblasts indicate oxidative stress status and may justify antioxidant therapy in OXPHOS disorders. Biochimica et biophysica acta, 2012. 1817(11): p. 1971-8.

44. Mari, M., et al., Mitochondrial glutathione, a key survival antioxidant. Antioxidants \& redox signaling, 2009. 11(11): p. 2685-700. 
45. Williams, M.D., et al., Increased oxidative damage is correlated to altered mitochondrial function in heterozygous manganese superoxide dismutase knockout mice. The Journal of biological chemistry, 1998. 273(43): p. 28510-5.

46. Hosoki, A., et al., Mitochondria-targeted superoxide dismutase (SOD2) regulates radiation resistance and radiation stress response in HeLa cells. Journal of radiation research, 2012. 53(1): p. 58-71.

47. Vergani, L., et al., Antioxidant defences and homeostasis of reactive oxygen species in different human mitochondrial DNA-depleted cell lines. European journal of biochemistry / FEBS, 2004. 271(18): p. 3646-56.

48. Leist, M., et al., Conventional cell culture media do not adequately supply cells with antioxidants and thus facilitate peroxide-induced genotoxicity. Free Radic Biol Med, 1996. 21(3): p. 297-306.

49. Taguchi, K., H. Motohashi, and M. Yamamoto, Molecular mechanisms of the Keap1-Nrf2 pathway in stress response and cancer evolution. Genes to cells : devoted to molecular \& cellular mechanisms, 2011. 16(2): p. 123-40.

50. Halliwell, B., Oxidative stress in cell culture: an under-appreciated problem? FEBS Lett, 2003. 540(1-3): p. 3-6.

51. Zannella, V.E., et al., Reprogramming metabolism with metformin improves tumor oxygenation and radiotherapy response. Clinical cancer research : an official journal of the American Association for Cancer Research, 2013. 19(24): p. 6741-50.

52. Koritzinsky, M., Metformin: A Novel Biological Modifier of Tumor Response to Radiation Therapy. International journal of radiation oncology, biology, physics, 2015. 93(2): p. 454-64. 



\section{Chapter}

\section{Mitochondrial dysfunction inhibits hypoxia- induced HIF-1 $\alpha$ stabilization and expression of downstream targets}

Marike W. van Gisbergen, Kelly Offermans, An M. Voets, Natasja G. Lieuwes, Rianne Biemans, Roland F. Hoffmann, Ludwig Dubois*, Philippe Lambin*

* contributed equally 


\section{Abstract}

Background and objective: For several years evidence is accumulating that not only more eminent known nuclear DNA mutations but also different variations (e.g. deletions and mutations) in the mitochondrial DNA (mtDNA) are associated with a wide variety of cancers. These mtDNA variations often result in a bioenergetic dysfunction of mitochondria causing the tumor cell to make a metabolic switch towards glycolysis resulting in an unbalanced $\mathrm{pH}$ homeostasis. In hypoxic cells, expression of the tumorassociated carbonic anhydrase 9 (CAIX), which is transcriptionally regulated by HIF-1 $\alpha$, is enhanced to maintain cellular $\mathrm{pH}$ homeostasis. Therefore, we hypothesized that cells with dysfunctional OXPHOS display elevated CAIX expression levels.

Material/methods: A549 (non-small cell lung carcinoma) and 143B (osteosarcoma) parental and mtDNA depleted cell lines $\left(\rho^{0}\right)$ were used. Additionally, cytoplasmic hybrids (cybrids) were produced by fusing the mtDNA-deprived 143B cell line with mtDNA derived from patient fibroblasts with the m.3243A>G MELAS mutation and were metabolically characterized using the Seahorse XF96 analyzer to determine mitochondrial respiration and glycolysis. OXPHOS complex I (CI) inhibition was achieved by exposing cells to metformin $(5 \mathrm{mM})$ or rotenone $(1 \mu \mathrm{M})$ for 16 hours. CAIX, VEGF, HIF-1 $\alpha$ and PHD2 mRNA expression was determined by qPCR and CAIX and HIF-1 $\alpha$ protein levels were determined by Western blotting. Reactive oxygen species levels were determined by DHR flow cytometry. Tumor growth, time to reach $500 \mathrm{~mm}^{3}$ and hypoxic fraction (pimonidazole and CAIX positivity) was monitored for xenografts generated from the cytoplasmic hybrids.

Results: The MELAS cybrid model showed a reduced mitochondrial respiration compensated by an increased glycolysis. CAIX mRNA and protein expression levels upon hypoxia $\left(0.2 \% \mathrm{O}_{2}\right)$ were blocked upon pharmacological or genetic OXPHOS inhibition. Also, VEGF (mRNA) and HIF-1 $\alpha$ (protein) expression levels were reduced. Levels of ROS were lowered for the 143B mtDNA depleted cells but remained similar for the cybrid model upon hypoxia exposure. PHD2 expression (mRNA) levels were not affected. Hypoxia levels were decreased in vivo for the MELAS compared to control cybrid xenografts.

Conclusion: Our results demonstrated that OXPHOS inhibition, either genetically or pharmacologically, leads to a decreased HIF-1 $\alpha$ stabilization and expression of downstream targets like CAIX and VEGF. Inhibition of mitochondrial function is therefore an interesting approach to increase therapeutic efficacy. 


\section{Introduction}

As the tumor microenvironment is a heterogeneous and dynamic entity, cells within a tumor can have different gene expression profiles, metabolism and oxygen supply. The lack of nutrient and oxygen supply is associated with two main characteristics of a malignancy, namely bioenergetics and angiogenesis [1]. The adaptation of cancer cells to hypoxia, the low oxygen regions within a tumor, is critical for tumor progression and is regulated by the hypoxia-inducible factor-1 (HIF-1) [2]. In addition to its role in proliferation, survival, angiogenesis and metastasis, HIF-1 is also involved in the regulation of tumor cell metabolism. During hypoxic stress, HIF-1 is able to reprogram tumor cell metabolism towards the low yield-energy production of the glycolytic pathway [3], which is associated with an increase in glucose uptake and lactate release [4]. HIF-1 reprograms metabolism not only by the up-regulation of glycolysis itself [5] but also by down-regulation of the mitochondrial respiration through regulating for instance pyruvate dehydrogenase kinase 1 expression [6, 7]. Transport of lactate, a byproduct of the glycolysis, into the microenvironment leads to extracellular acidification.

One important family of proteins involved in tumor $\mathrm{pH}$ regulation are the carbonic anhydrases (CA). One of these CA members is the membrane bound zinc-metalloenzyme CAIX, which is capable of re-hydrating $\mathrm{CO}_{2}$ into bicarbonate $\left(\mathrm{HCO}_{3}{ }^{-}\right)$and a proton $\left(\mathrm{H}^{+}\right)$upon passive $\mathrm{CO}_{2}$ diffusion out of the cell. $\mathrm{HCO}_{3}{ }^{-}$will subsequently re-enter the cell in order to neutralize the intracellular $\mathrm{pH}$. The remaining proton remains in the extracellular environment and contributes to extracellular acidification, which is related to worse prognosis in cancer patients $[8,9]$. High CAIX expression results in a higher risk of locoregional failure, disease progression and metastases development in cancer patients [10]. In hypoxic tumors CAIX expression is found to be upregulated through transcriptional activation upon interaction of HIF-1 $\alpha$ with the hypoxia response element (HRE) identified in its promotor region [11]. Also, the transport of lactate into the microenvironment leads to an increase in extracellular acidification. The role of the dysfunctional mitochondria in these mechanisms is of interest. It has been suggested that dysfunction of the oxidative phosphorylation machinery contributes to the tumoral metabolic reprogramming $[12,13]$. Therefore, we hypothesized that mitochondrial dysfunction as a result of mtDNA variations would potentially lead to an increased expression of CAIX. 


\section{Material and methods}

\section{In vitro models}

mtDNA depleted cells

The parental and mtDNA depleted (rho-zerol $\rho^{0}$ )143B osteosarcoma cell lines were kindly provided by Dr. Valeria Tiranti (Milan, Italy). Both cell lines were cultured in Gibco's Dulbecco's modified Eagle's medium (DMEM) with $10 \%$ fetal bovine serum (FBS; Sigma-Aldrich). Culture medium of the mtDNA depleted cells was supplemented with $150 \mu \mathrm{g} / \mathrm{ml}$ uridine (Acros Organics) and $100 \mu \mathrm{g} / \mathrm{ml}$ bromodeoxyuridine (SigmaAldrich). The parental and mtDNA depleted A549 (alveolar type-II carcinoma) cell lines were cultured in DMEM supplemented with 25\% FBS, vitamins, amino acids (SigmaAldrich) and uridine (50 $\mathrm{\mu g} / \mathrm{ml}$; Acros Organics). mtDNA depletion was sustained by culturing cells in the medium supplemented with ethidium bromide $(50 \mathrm{ng} / \mathrm{ml}$; SigmaAldrich). Fluorescent confocal imaging and quantitative PCR were used to confirm mtDNA depletion. For confocal imaging, cells were stained (45 min) with MitoTracker ${ }^{\circledR}$ Deep-Red FM and Picogreen (Invitrogen) in D-PBS (GIBCO) according to manufacturer protocol. Subsequently, cells were washed twice with D-PBS and visualized using a Leica AOBS confocal microscope. Overlays were generated using Image J. Ratios of nDNA (B2M) and mtDNA (D-Loop) were obtained by quantitative PCR in order to determine the mtDNA copy numbers. DNA was isolated using the gentra puregene kit (Qiagen). Quantitative PCR was performed on the $7900 \mathrm{HT}$ Fast Real-Time PCR System (Applied Biosystems). Gene abundances were detected using SensiMix SYBR ${ }^{\circledR}$ HiRox kit (Bioline Reagents). The cycling conditions were: $2^{\prime} 50^{\circ} \mathrm{C}, 10^{\prime} 95^{\circ} \mathrm{C}, 40$ cycles of $15^{\prime \prime}$ at $95^{\circ} \mathrm{C}+1^{\prime}$ $60^{\circ} \mathrm{C}$. Primer sequences for B2M and D-Loop can be found in supplementary Table 1.

Cytoplasmic hybrids (cybrids) and quantification of mutant mtDNA percentage Cytoplasmic hybrids cell lines (cybrids) were generated as previously described [14, 15]. In short, cybrids were produced by fusing the mtDNA from patient fibroblasts with the mtDNA depleted 143B cell line. Two control cybrid cell lines were generated using fibroblast mtDNA from healthy volunteers (kindly provided by Dr. Hubertus Smeets, Maastricht, the Netherlands), while 2 mutant cybrid cell lines were created using fibroblasts harboring the m.3243A>G mutation encoding for mitochondrial tRNALeu(UUR) (MT-TL1) and responsible for the "mitochondrial encephalomyopathy, lactic acidosis, and stroke-like episodes" (MELAS) syndrome [16]. All cell lines were cultured in DMEM supplemented with 5\% dialyzed serum (Invitrogen). To determine the percentage of mtDNA harboring the MELAS m.3243A>G mutation, the coding mtDNA sequence was amplified using primer sequences found in supplementary Table 1 . The PCR amplification consisted of an initial denaturation at $94^{\circ} \mathrm{C}(5 \mathrm{~min})$, followed by 32 cycles at $92^{\circ} \mathrm{C}(1 \mathrm{~min}), 53^{\circ} \mathrm{C}(1 \mathrm{~min}), 72^{\circ} \mathrm{C}(45 \mathrm{~s})$ and a final elongation at $72^{\circ} \mathrm{C}(7 \mathrm{~min})$. A FAM labeled forward primer was added to the first PCR product which was subjected to 
an additional PCR cycle. Gel electrophoresis (2\% agarose) confirmed size of the PCR products. Fragments were excised from the gel and digested $\left(2.5 \mathrm{~h}\right.$ at $\left.37^{\circ} \mathrm{C}\right)$ using a mix of sterile water, digestion buffer and the restriction enzyme Hae III. Digestion products were purified using the QiaQuick PCR Purification kit (Qiagen) and analyzed with ABI 3730 using a G5 filter. The primer sequences for fragment analysis can be found in Table S1.

\section{Metabolic profiling}

Metabolic profiles were generated using the Seahorse XF96 extracellular Flux analyzer (Agilent) according to manufacturer's guidelines [17]. Cells were seeded at an optimized cell density of 30.000 cells/well. The glycolysis stress test was performed by sequential addition of $10 \mathrm{mM}$ glucose, optimized oligomycin concentration (2.5 $\mu \mathrm{M})$ and $0.1 \mathrm{M} 2$ deoxyglucose (2-DG) (Sigma-Aldrich).

OXPHOS inhibition

500.000 cells of each cell line were seeded in $6 \mathrm{~cm}$ centimeter dishes. Cells were exposed to either normoxia $\left(20 \% \mathrm{O}_{2}\right)$ or hypoxia $\left(0.2 \% \mathrm{O}_{2}\right)$ for 16 hours simultaneously with incubation of vehicle, $5 \mathrm{mM}$ metformin (Sigma-Aldrich) or $1 \mu \mathrm{M}$ rotenone (Sigmaaldrich). After 16 hours, cells were immediately put on ice and were washed with PBS prior to protein or RNA isolation.

DNA isolation, RNA isolation and quantitative PCR analysis

mRNA was extracted using NucleoSpin RNA II kit (Bioke) and reverse transcribed using iScript cDNA Synthesis Kit (BioRad). All procedures were according to the manufacturers' instructions. Quantitative RT-PCR was performed in the ABI 7700 (Applied Biosystems) or 7900HT Fast Real-Time PCR. Gene abundances were detected with SYBR ${ }^{\circledR}$ Green (Eurogentec). mRNA expression was normalized to 18S. Primer sequences can be found in Table S1.

\section{SDS-PAGE and Western blotting}

Cells were lysed and processed as described previously [18]. Antibodies used were M75 (1:40) (kindly provided by Silvia Pastorekova, Institute of Virology, Slovak Academy of Science, Bratislava, Slovak Republic) against CAIX, anti-HIF-1 $\alpha$ (1:250, BD transduction) and anti- $\beta$-actin (1:200.000, MD biosciences). Proteins were visualized by the horseradish peroxidase method (anti-mouse 1:2000, Cell Signaling) by using ECL prime western blotting detection reagent (Amersham Corp) or ECL supersignal west pico (Thermo Fisher). 
Proliferation assay

2500 cells/well were seeded in a 96-wells plate and allowed to settle overnight. Subsequently, proliferation was monitored during 7 days in the IncuCyte FLR (Essen Bioscience) or until $>90 \%$ confluency was observed.

Flow cytometry.

Formation of reactive oxygen species (ROS) was detected 16 hours after hypoxia or normoxia exposure. Cells were incubated with $20 \mu \mathrm{M}$ dihydrorhodamine-123 during the last hour of exposure. ROS levels were determined in the propidium iodide (SigmaAldrich) negative population by flow cytometry (BD FACS Canto II).

Hypoxia tolerance

Cells were seeded in $6 \mathrm{~cm}$ dishes, allowed to attach under normoxic conditions overnight and exposed to hypoxic conditions $\left(0.2 \% \mathrm{O}_{2}\right)$ for 24,48 or 72 hours. After hypoxic exposure, medium was replaced and cells were allowed to form colonies under normoxia for 7 days. Colonies were quantified after staining and fixation with $0.4 \%$ methylene blue in $70 \%$ ethanol. A colony was defined as $>50$ cells.

\section{In vivo models}

All experiments were in accordance with local institutional guidelines for animal welfare and were approved by the Animal Ethics Committee of the University. Control and mutant cybrid cells $\left(1.5^{*} 10^{6}\right)$ were resuspended in Basement Membrane Matrix (Matrige ${ }^{\mathrm{TM}}$ BD Biosciences) and injected subcutaneously into the lateral flank of adult NMRI nu/nu mice (28-30 g). Tumor growth was monitored until reaching a volume of $1.2 \mathrm{~cm}^{3}$.

Immunohistochemisty

Frozen cybrid xenograft tumors were sectioned $(5 \mu \mathrm{m})$ and stained for hypoxia (pimonidazole) and CAIX. Sections were fixed using cold acetone, rehydrated in TBS with 0.2\% Tween-20 (TBS-T) and pre-incubated with 1\% normal goat serum (NGS) before exposing them to the primary antibodies: rabbit anti-pimonidazole (1:150, HP3-1000, Bio-connect) and rabbit anti-CAIX (1:1000, Novus biologicals). After washing with TBS-T, incubation with the secondary antibody goat anti-rat Alexa488 (1:750) and goat antirabbit Alexa 594 (1:500) (Invitrogen) was performed. Sections were mounted using fluorescent mounting medium (DakoCytomation) and digitally scanned using an Olympus BX51WI fluorescence microscope with a Hamamatsu EM-CCD C9100 digital camera, a motorized stage (Ludl Mac 2000) and a 10x objective. Micromanager 1.4 software was used for automated image acquisition. Image stitching was performed by using ImageJ software. 


\section{Statistics}

All statistical analyses were performed with GraphPad Prism (GraphPad Software, version 5.03, 2009, California, USA). A non-parametric Mann-Whitney $U$ test for small groups was used to determine the statistical significance of differences between two independent groups of variables. A p-value $<0.05$ was considered significant.

\section{Results}

In order to investigate if extreme changes in oxidative phosphorylation (OXPHOS) capacity would lead to a change in CAIX expression, we used A549 and 143B mtDNA depleted cancer cell lines ( $\rho^{0}$ cells). Both cell lines showed a substantial decrease of mtDNA in mitochondria (Fig. 1A) which is confirmed by the significant $(p<0.05)$ decrease in mtDNA copy number (Fig. 1B). CAIX expression was decreased under hypoxic conditions $(p<0.05)$ in the cells depleted from their mtDNA, on both mRNA (Fig. 1C) and protein levels (Fig. 1D).

A

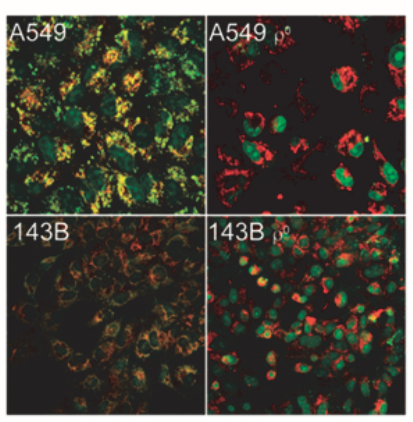

C

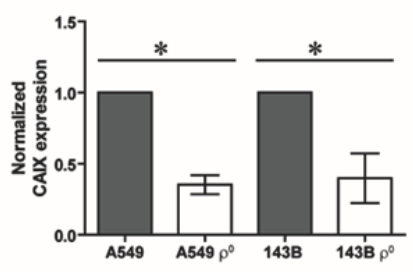

B

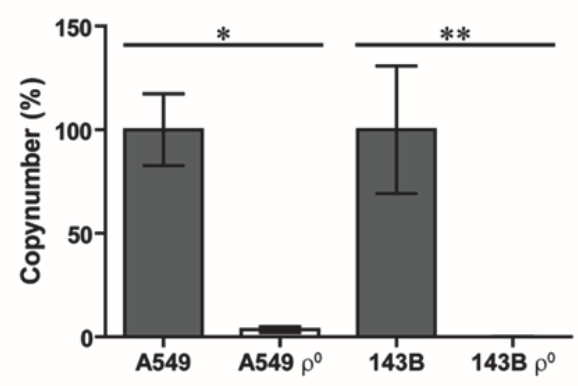

D
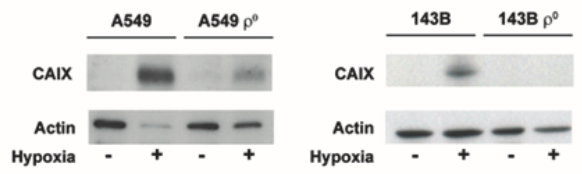

Figure 1. Generation and characterization of mtDNA depleted cell lines.

A. Merged images of dsDNA staining (green) and mitochondria (red). Yellow areas contain both mitochondria and dsDNA. B. Percentage mtDNA copy number. C. CAIX mRNA expression upon hypoxia and D. CAIX protein expression. Data represent the mean + /- SEM of at least 3 biological repeats. ${ }^{*} p<0.05,{ }^{* *} p<0.01$. 
Our observations were extended to a second model, in which mtDNA is not abolished but harbors a point mutation, the so-called cytoplasmic hybrid or cybrid model. For this purpose, the mtDNA depleted 143B osteosarcoma cell line was fused with the cytoplasts from a fibroblast cell line from a MELAS patient. The mutant cybrid polyclonal cell line MELAS 1 had an average mutation load of 96.80\%, while the control cybrid clones contained 99.85 (control 1) and $99.65 \%$ (control 2) of the wild-type sequence. MELAS 2 cells showed a mutation load of $82.33 \%$ (Fig. 2A). To further characterize the cybrid models, metabolic respiration was investigated by assessing mitochondrial respiration and glycolytic capacity. Basal respiration of the MELAS 1 cells was significantly $(p<0.05)$ reduced compared to the control lines (Fig 2B). MELAS 2 cells, displayed an increase is mitochondrial respiration compared to MELAS 1 cells (Fig 2B). Where MELAS 1 cells were completely dependent on glycolysis, since no glycolytic reserve capacity could be observed (Fig 2B), MELAS 2 did display a glycolytic reserve capacity with comparable levels to the control cell lines, suggesting that the OXPHOS was still functional in these cells. Baseline respiration and glycolysis was similar for both control cybrid lines, therefore control 1 was selected for subsequent experiments. Upon hypoxia CAIX mRNA and protein expression was abolished in MELAS 1 cells (Fig. 2C-D). For MELAS 2 mutants harboring a lower mutation percentage this effect was rescued (Fig. S1) and therefore these mutant cells were excluded from further experiments. MELAS mutation leads to a reduction in activity of Complex I - IV of the oxidative phosphorylation chain [16]. In order to investigate if the observed effects from the genetic approach could be mimicked by pharmacological inhibition, wild-type cells were exposed to either metformin or rotenone, both complex I $(\mathrm{Cl})$ inhibitors. Metformin exposure decreased proliferation rate in the control cybrid line, while no effect was observed for the MELAS 1 mutants. The doubling increased with $27 \%(p<0.05)$ for control cybrid line 1. Similarly, an increased doubling time was observed for 143B $(p<0.05)$ and A549 $(p<0.0001)$ parental lines upon metformin exposure (Fig. 2E). Pharmacological $\mathrm{Cl}$ inhibition resulted in reduced CAIX mRNA and protein expression upon hypoxia exposure in the $143 \mathrm{~B}$ parental cells (rotenone $\mathrm{p}<0.05$ ) and the control cybrid line (metformin $p<0.05$ and rotenone $p<0.05$ ). CAIX expression was not reduced for the A549 cells (Fig. 2F-G).

Additionally, VEGF mRNA expression, another HIF-1 $\alpha$ downstream target was also decreased (Fig. 3A). HIF-1 $\alpha$ protein expression upon hypoxia was reduced for the parental lines and the control cybrid line after exposure to metformin. HIF-1 $\alpha$ protein expression was in general low for the mitochondrial dysfunction A549 $\rho^{0}$ and the MELAS 1 mutant cells (Fig. 3B), while HIF-1 a mRNA expression was not affected (Fig. 3C). 
A

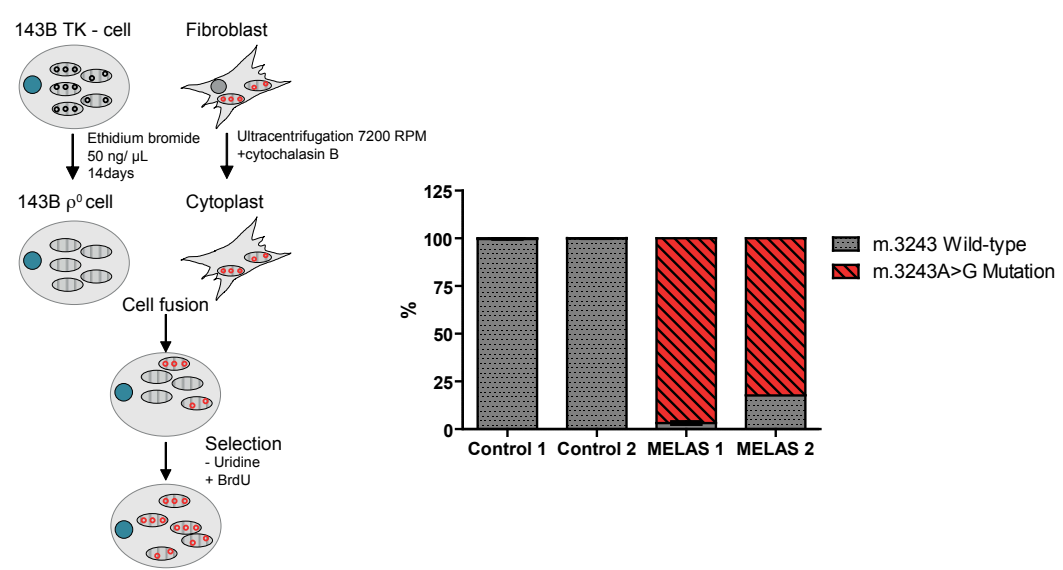

B

Basal respiration

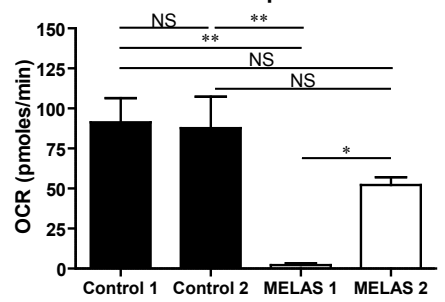

C

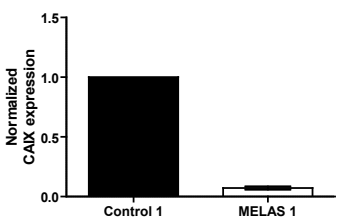

D
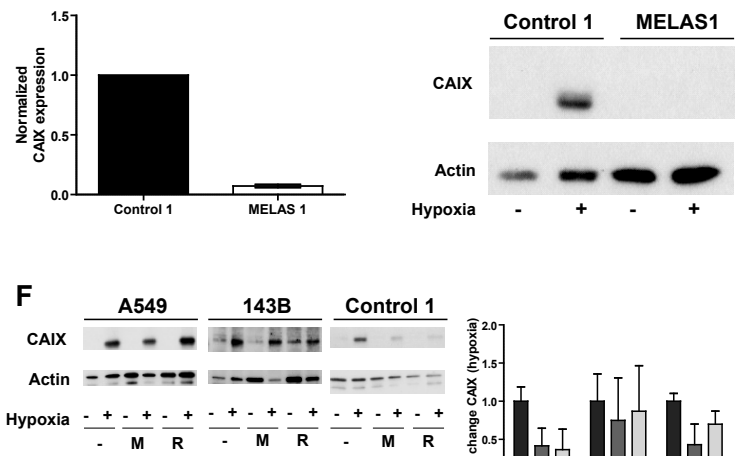

Glycolysis stress test

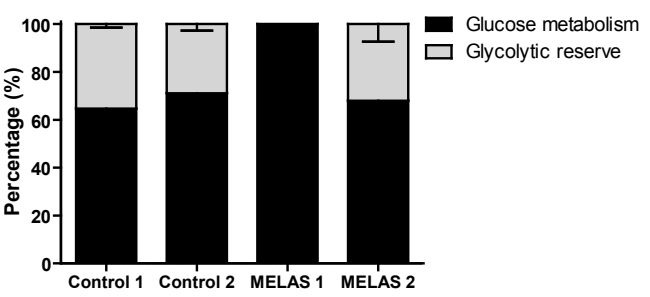

E
G

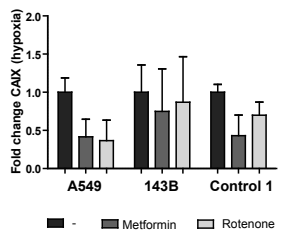

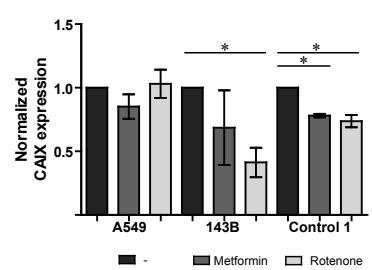

Figure 2. Generation and characterization of the cybrid model and influence of pharmacological $\mathrm{Cl}$ inhibition on CAIX expression.

A. Procedure to generate MELAS and control cybrid cell lines (left) and percentage m.3243A>G mutated mtDNA in these cybrid lines (right). B. Metabolic profiling of the cybrid lines, showing basal respiration (left) and glycolysis stress test results (right). C. Normalized CAIX mRNA expression upon hypoxia. D. Representative 
Western blot of CAIX protein expression upon normoxia (-) or hypoxia (+). E. Normalized doubling times upon metformin. F. CAIX protein expression upon normoxia (-) or hypoxia (+) with or without Metformin (5mM) or Rotenone (1UM). Data represents mean + SEM of 2 independent biological repeats. G. CAIX mRNA expression upon hypoxia with or without Metformin ( $5 \mathrm{mM})$ or Rotenone (1uM). All data (B-E,G) represents the mean $+/-$ SEM of at least 3 independent biological repeats. ${ }^{*} p<0.05 * * p<0.01$.

A

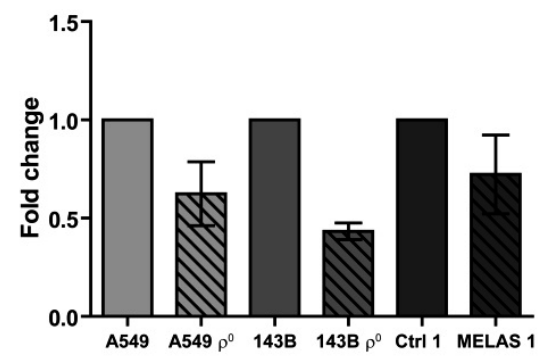

B
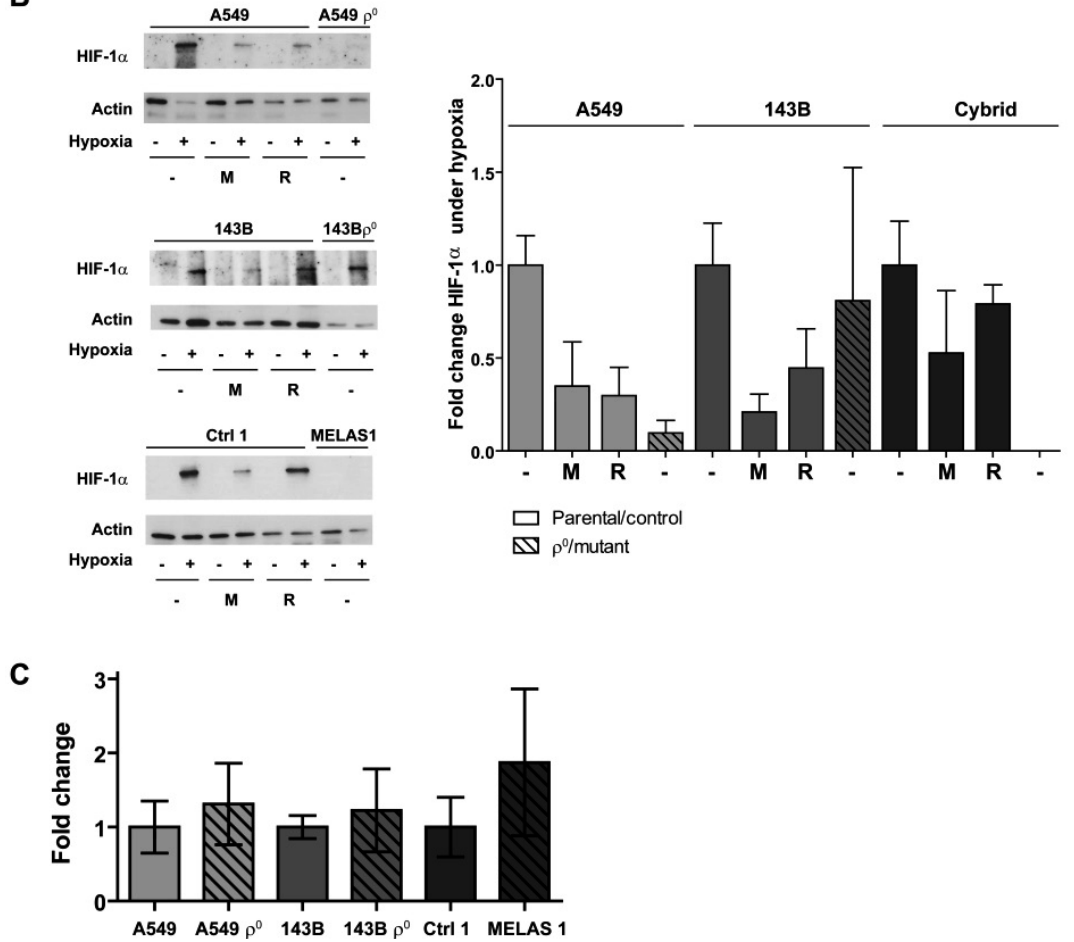

Figure 3. HIF-1 $\alpha$ expression and other downstream targets.

A. VEGF mRNA expression under hypoxia. Data represents the mean of at least 3 independent biological repeats +/- SEM. B. Representative Western blot of HIF-1a protein expression upon normoxia (-) or hypoxia (+), followed by exposure of vehicle (-); metformin (M) and rotenone (R). Quantification of the data is shown in the right panel. Data represents the mean of at least 2 independent biological repeats +/- SEM. C. HIF-1 $\alpha$ mRNA expression under hypoxia. Data represents the mean of at least 2 independent biological repeats +/- SEM. 
PHD2 is the main regulator of HIF-1 $\alpha$ stabilization [19-21]. Therefore, we investigated if changes in PHD2 expression could explain the observed reduction in HIF-1 $\alpha$ stabilization and expression of downstream targets. PHD2 mRNA expression was not changed upon genetic imposed mitochondrial dysfunction (Fig. 4A, C). Similarly, mRNA expression of PHD1 and 3 did not alter (data not shown). Another major contributor to HIF-1 $\alpha$ stabilization is the presence of ROS production $[22,23]$. Contradictory results have been reported on the influence of mtDNA variations on ROS production, which can be increased $[16,24]$ or decreased $[25,26]$. Hypoxia induced ROS production 1.5 to 2 fold for cybrid and $\rho^{0}$ cells respectively. Overall, ROS production decreased for the mtDNA depleted 143B cells, while no differences were observed for the cybrid cells (Fig. 4B, D). Additionally, pharmacological inhibition was not able to change ROS production.

A

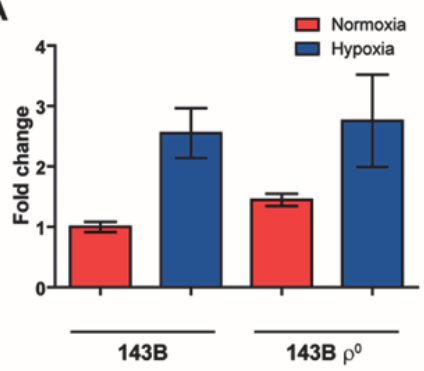

C

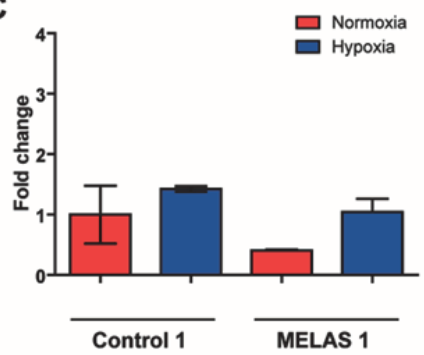

B

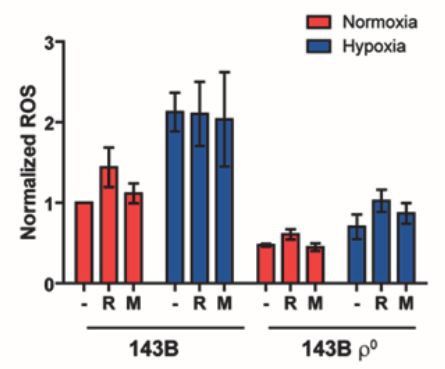

D

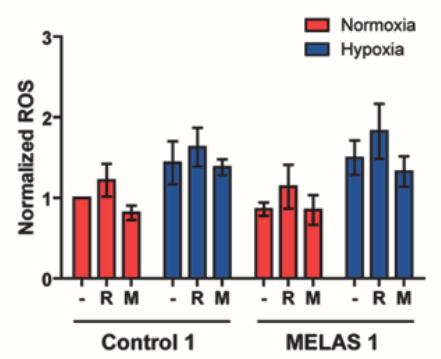

Figure 4. HIF-1 $\alpha$ regulators.

A, C. PHD2 mRNA expression. Data are normalized to either 143B parental (A) or ctrl 1 cybrid cell lines (C). Data represents the mean of at least 2 independent biological repeats +/- SEM. B, D. Normalized ROS production upon mtDNA depletion (B) or mtDNA mutation (D) under normoxic or hypoxic conditions with or without OXPHOS Cl inhibitors. Data represents the mean of at least 3 independent biological repeats +/- SEM.

In order to investigate the functional consequences of decreased CAIX expression we assessed clonogenic survival upon hypoxic conditions. Survival under prolonged hypoxia for parental and mtDNA depleted 143B cells was not altered, while decreased for the cybrid models with a tendency of lower survival for the control cybrid line (Fig. 5A). In vivo, MELAS cybrid xenografts need longer to reach $500 \mathrm{~mm}^{3}$ compared to control 
cybrid tumors, however no differences were found in tumor doubling time suggesting a delayed tumor take (Fig. 5B). Additionally, the mutation percentage for the mutant hybrid xenografts at sacrifice decreased compared with the injected cells (Fig. 5B). HIF$1 \alpha$ protein was reduced in the mutant hybrid xenografts compared with control tumors, however a large variation was observed. Additionally, we observed that in the cytoplasmic hybrids cells harboring a MELAS mutation hypoxia, evaluated by pimonidazole was reduced. However, no large differences upon CAIX staining could be noticed (Fig. 5C).

\section{A}
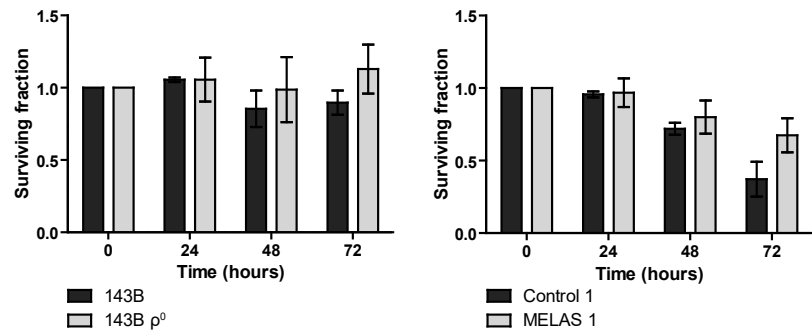

B
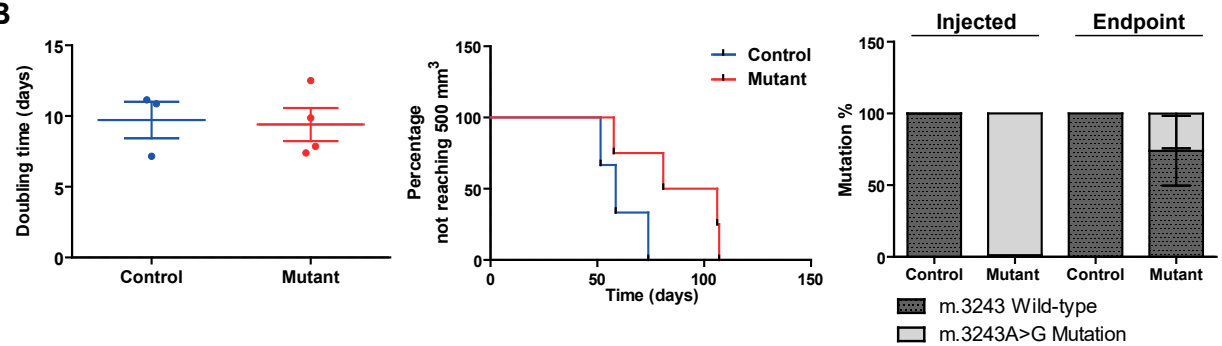

C
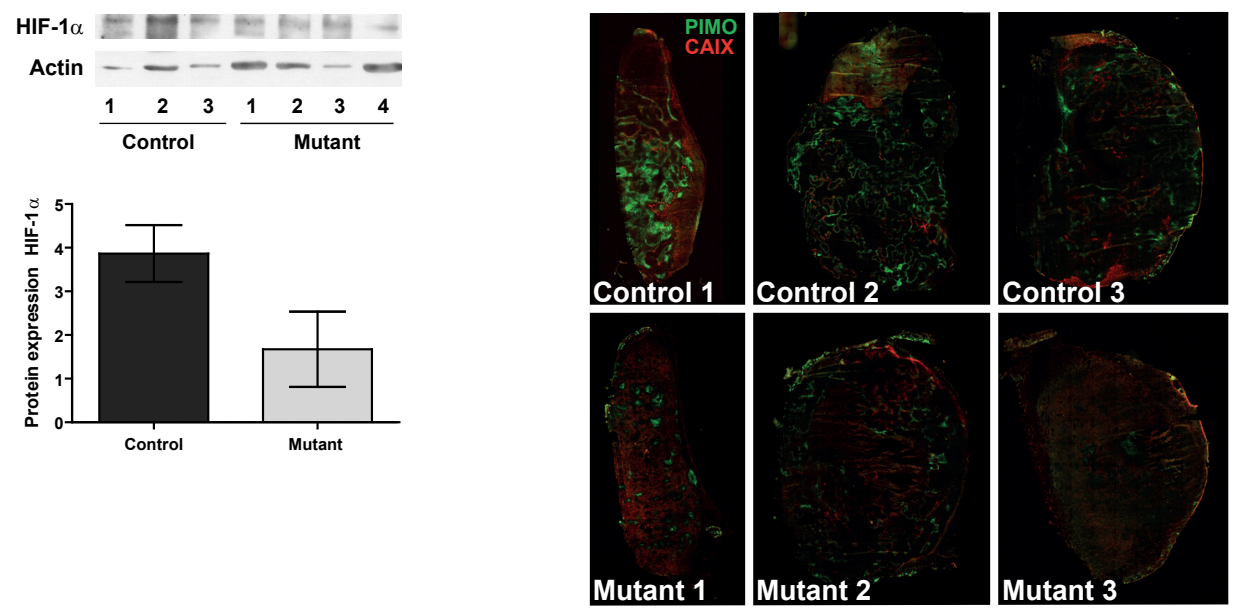
Figure 5. Functional consequenses of mtDNA mutations in relation to hypoxia.

A. Hypoxia tolerance. Data represents the mean of at least 3 independent biological repeats +/- SEM. B. Doubling time (left panel), percentage not reaching $500 \mathrm{~mm}^{3}$ (middle panel) and mutation percentage before injection and after tumor excision (right panel). C. Protein expression of HIF-1 $\alpha$ and actin (left). Immunohistochemisty (right) of hypoxia assessed using pimonidazole (green) and CAIX (red).

\section{Discussion}

In the present study, the effect of OXPHOS inhibition using genetic models (mtDNA depleted cells and mtDNA mutated cytoplasmic hybrids) or pharmacological inhibitors (rotenone, metformin) on CAIX expression and HIF-1 $\alpha$ stabilization was investigated. We hypothesized that a decrease of mitochondrial respiration through oxidative phosphorylation (OXPHOS) would lead to an increase in CAIX expression upon hypoxia exposure. However, contradicting our hypothesis we observed that CAIX expression was reduced upon low oxygen tensions, with reduced HIF-1 $\alpha$ levels in cells with mitochondrial dysfunction. Since the tumor microenvironment is a heterogeneous and dynamic mechanism, cells within a tumor can have different gene expression profiles, metabolism and oxygen supply [27-29]. The adaptation of cancer cells to for example hypoxia is critical for tumor progression and metastasis formation and is regulated by the hypoxia-inducible factor-1 (HIF-1)[30-33]. Under reduced oxygen conditions normally HIF-1 $\alpha$ is stabilized $[34,35]$. Mitochondrial ROS production seems to be necessary for HIF-1 $\alpha$ stabilization [36], even when a functional OXPHOS is not present [37]. In addition to its role in proliferation, survival, angiogenesis and metastasis, HIF-1 is also involved in the regulation of tumor cell metabolism [34, 38, 39]. Studies have described an association between HIF-1 and mitochondrial function. For instance it has been shown that BAY 87-2243, a potent inhibitor of HIF-1a, reduced tumor growth, potentially through targeting mitochondrial $\mathrm{Cl}[40,41]$.

During hypoxic stress, the low efficiency glycolytic pathway is further upregulated, mainly driven by HIF-1 $\alpha$. Similarly, a shift towards the glucose metabolism is induced upon mitochondrial dysfunction (Fig. 2B). The high rate of glycolysis, which persists in tumor cells even under re-oxygenation, is associated with an increase in glucose uptake and lactate release by the cells. Transport of lactate into the microenvironment leads to an enhanced extracellular acidification. Lactic acidosis is a symptom that is often observed in patients with mitochondrial diseases like myoclonic epilepsy with raggedred fibers (MERFF) syndrome [44]. A cybrid model harboring a m.8344A>G mutation encoding for ND5 subunit of $\mathrm{Cl}$, corresponding to the MERFF phenotype in patients resulting in $\mathrm{Cl}$ dysfunction, displayed reduced levels of carbonic anhydrase VIII (CAVIII) [45]. Furthermore, it has been suggested that CAVIII regulates stress responses in the cell and could also play a role in metabolism. CAVIII knockdown cells were found to have a decreases glycolytic activity and increased cell death under reduced glucose 
concentrations in vitro [46]. These data suggest that mitochondrial dysfunction leading to decreased carbonic anhydrase expression levels could be translated to other CA forms. One of these isoforms is the membrane bound carbonic anhydrase IX (CAIX) which has been involved in maintaining a physiological intracellular $\mathrm{pH}$ [47]. In our study, we observed in a MELAS cybrid model (m.3243A>G encoding for MT-TL1) that indeed CAIX mRNA and protein expression was reduced under hypoxic conditions. Although the data on $\mathrm{Cl}$ inhibition were not always as clear as for the genetical cellular models, the differences in effects could probably be explained by the differences between cell lines.

Our findings show that the time for tumor take was longer for mutant hybrid xenografts, but growth rates were not altered once the tumor was established compared to control tumors. Previously it has been shown that HIF-1 $\alpha$ is responsible for tumor establishment, since HIF-1 $\alpha$ knockdown resulted in a longer time needed to reach $60 \mathrm{~mm}^{3}$. However, once tumors were established no difference in tumor growth rate was observed [48]. In agreement, HIF-1 $\alpha$ expression levels were reduced for the mutant hybrid xenografts. Furthermore, most of the tumors established from the mutant cell line lost their m.3243A>G mutation in vivo, possibly explaining the tumor take as we observed. The presence of HIF-1 $\alpha$ in cell lines with lower mutation percentage supports this observation.

Another possible explanation of the absence of HIF-1 $\alpha$ might be the involvement of the AMPK-mTOR pathway, which regulated energetic stress responses under normoxic conditions, as for instance the $\mathrm{Cl}$ inhibitor metformin used in our experiments causes inhibition of mTOR $[49,50]$. However additional experiments are necessary to evaluate if OXPHOS inhibition is inducing alterations in AMPK-mTOR signaling and results in this observed HIF-1 $\alpha$ deregulation by altered mRNA translation. Alterations in general mRNA translation are influenced upstream by the unfolded protein response (UPR) a mechanism influenced by hypoxia and energetic stress [51-53]. Not only general UPR responses but also the mitochondrial UPR response could possibly be an interesting mechanism. One of the regulators influencing this mitochondrial UPR is NAD-dependent deacetylase sirtuin-3 (SIRT3) [54]. SIRT3 is located in the mitochondria and is involved in various cellular mechanisms such as nutrient stress [55], fatty acid oxidation [56], AMPK activation [57], antioxidant mechanisms [58] and in the activation of a hypoxia-induced mitochondrial form of autophagy (mitophagy) [59]. Additionally HIF-1 $\alpha$ stabilization under hypoxic conditions is suppressed under the influence of SIRT3 $[60,61]$ however, other SIRT enzymes can influence the functionality of HIF-1 $\alpha[62,63]$. Therefore, we suggest that SIRT3 expression causes the destabilization of HIF-1 $\alpha$ and mitophagy and/or autophagy whereas upregulation of autophagy was observed in the CA8 mutant cell lines under normoxic conditions [45], although these relationships have to be further investigated. 


\section{Conflict of interest statement}

The authors have no conflict to declare.

\section{Acknowledgements}

This research was financially supported the Netherlands Genomics Initiative (pre-seed grant 2012, 93612005). 


\section{Supplementary data}

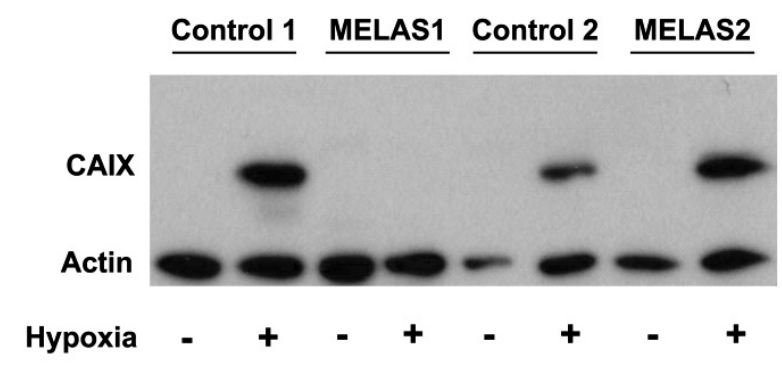

Supplementary Figure S1. CAIX Expression for control and MELAS cybrid lines upon exposure to hypoxia.

Supplementary Table 1: The primer sequences for quantitative real-time PCR and fragment analysis.

\begin{tabular}{lll}
\hline Target gene & Forward primer & Reverse primer \\
\hline D-loop & 5'-CAT CTG GTT CCTA CTT CAG GG-3' & 5'-TGA GTG GTT AAT AGG GTG ATA GA-3' \\
B2M & 5'-TGC TGT CTC CAT GTT TGA TGT ATC T-3' & 5'- TCT CTG CTC CCC ACC TCT AAG T-3' \\
MELAS & 5'-CAA CTT AGT ATT ATA CCC ACA C-3' & 5'-TTT CGT TCG GTA AGC ATT AG-3' \\
MELAS-FAM & FAM-5'-CAA CTT AGT ATT ATA CCC ACA C-3' & \\
CAIX & 5'-CAT CCT AGC CCT GGT TTT TGG-3' & 5'-GCT CAC ACC CCC TTT GGT T-3' \\
VEGF & 5'-GAC TCC GGC GGA AGC AT-3' & 5'-TCC GGG CTC GGT GAT TTA-3' \\
HIF-1 $\alpha$ & 5'-ATC GCG GGG ACC GAT T-3' & 5'-CGA CGT TCA GAA CTT ATC TTT TTC TT-3' \\
PHD2 & 5'- GCA CGA CAC CGG GAA GTT-3' & 5'-ÇCA GTT CCC GTT ACA GT-3' \\
18S & 5'-AGT CCC TGC CCT TTG TACACA-3' & 5'-GAT CCG AGG GCC TCA CTA AAC-3'
\end{tabular}




\section{References}

1. Hanahan, D. and R.A. Weinberg, Hallmarks of cancer: the next generation. Cell, 2011. 144(5): p. 646-74.

2. McDonald, P.C., S.C. Chafe, and S. Dedhar, Overcoming Hypoxia-Mediated Tumor Progression: Combinatorial Approaches Targeting pH Regulation, Angiogenesis and Immune Dysfunction. Front Cell Dev Biol, 2016. 4: p. 27.

3. Denko, N.C., Hypoxia, HIF1 and glucose metabolism in the solid tumour. Nat Rev Cancer, 2008. 8(9): p. 705-13.

4. Neri, D. and C.T. Supuran, Interfering with $\mathrm{pH}$ regulation in tumours as a therapeutic strategy. Nature reviews. Drug discovery, 2011. 10(10): p. 767-77.

5. Semenza, G.L., et al., Transcriptional regulation of genes encoding glycolytic enzymes by hypoxiainducible factor 1. J Biol Chem, 1994. 269(38): p. 23757-63.

6. Kim, J.W., et al., HIF-1-mediated expression of pyruvate dehydrogenase kinase: a metabolic switch required for cellular adaptation to hypoxia. Cell Metab, 2006. 3(3): p. 177-85.

7. Papandreou, I., et al., HIF-1 mediates adaptation to hypoxia by actively downregulating mitochondrial oxygen consumption. Cell Metab, 2006. 3(3): p. 187-97.

8. Walenta, S. and W.F. Mueller-Klieser, Lactate: mirror and motor of tumor malignancy. Seminars in radiation oncology, 2004. 14(3): p. 267-74.

9. Blatt, S., et al., Lactate as a predictive marker for tumor recurrence in patients with head and neck squamous cell carcinoma (HNSCC) post radiation: a prospective study over 15 years. Clinical oral investigations, 2016. 20(8): p. 2097-2104.

10. van Kuijk, S.J., et al., Prognostic Significance of Carbonic Anhydrase IX Expression in Cancer Patients: A Meta-Analysis. Frontiers in oncology, 2016. 6: p. 69.

11. Wykoff, C.C., et al., Hypoxia-inducible expression of tumor-associated carbonic anhydrases. Cancer Res, 2000. 60(24): p. 7075-83.

12. Wallace, D.C., Mitochondria and cancer. Nat Rev Cancer, 2012. 12(10): p. 685-98.

13. Gaude, E. and C. Frezza, Defects in mitochondrial metabolism and cancer. Cancer Metab, 2014. 2: p. 10.

14. King, M.P. and G. Attardi, Human cells lacking mtDNA: repopulation with exogenous mitochondria by complementation. Science, 1989. 246(4929): p. 500-3.

15. King, M.P., et al., Defects in mitochondrial protein synthesis and respiratory chain activity segregate with the tRNA(Leu(UUR)) mutation associated with mitochondrial myopathy, encephalopathy, lactic acidosis, and strokelike episodes. Molecular and cellular biology, 1992. 12(2): p. 480-90.

16. Voets, A.M., et al., Patient-derived fibroblasts indicate oxidative stress status and may justify antioxidant therapy in OXPHOS disorders. Biochimica et biophysica acta, 2012. 1817(11): p. 1971-8.

17. Nicholls, D.G., et al., Bioenergetic profile experiment using C2C12 myoblast cells. Journal of visualized experiments : JoVE, 2010(46).

18. Dubois, L., et al., Imaging the hypoxia surrogate marker CA IX requires expression and catalytic activity for binding fluorescent sulfonamide inhibitors. Radiotherapy and oncology : journal of the European Society for Therapeutic Radiology and Oncology, 2007. 83(3): p. 367-73.

19. Metzen, E., et al., Regulation of the prolyl hydroxylase domain protein 2 (phd2/egln-1) gene: identification of a functional hypoxia-responsive element. The Biochemical journal, 2005. 387(Pt 3): p. 711-7.

20. Appelhoff, R.J., et al., Differential function of the prolyl hydroxylases PHD1, PHD2, and PHD3 in the regulation of hypoxia-inducible factor. The Journal of biological chemistry, 2004. 279(37): p. 38458-65.

21. Berra, E., et al., HIF prolyl-hydroxylase 2 is the key oxygen sensor setting low steady-state levels of HIF1alpha in normoxia. The EMBO journal, 2003. 22(16): p. 4082-90.

22. Niecknig, H., et al., Role of reactive oxygen species in the regulation of HIF-1 by prolyl hydroxylase 2 under mild hypoxia. Free radical research, 2012. 46(6): p. 705-17.

23. Bonello, S., et al., Reactive oxygen species activate the HIF-1alpha promoter via a functional NFkappaB site. Arteriosclerosis, thrombosis, and vascular biology, 2007. 27(4): p. 755-61. 


\section{Chapter 4}

24. Ishikawa, K., et al., ROS-generating mitochondrial DNA mutations can regulate tumor cell metastasis. Science, 2008. 320(5876): p. 661-4.

25. Chen, H., et al., Mitochondrial DNA depletion causes decreased ROS production and resistance to apoptosis. International journal of molecular medicine, 2016. 38(4): p. 1039-46.

26. Marin, J.J., E. Lozano, and M.J. Perez, Lack of mitochondrial DNA impairs chemical hypoxia-induced autophagy in liver tumor cells through ROS-AMPK-ULK1 signaling dysregulation independently of HIF1alpha. Free radical biology \& medicine, 2016. 101: p. 71-84.

27. $\mathrm{Hu}, \mathrm{J}$., et al., Heterogeneity of tumor-induced gene expression changes in the human metabolic network. Nature biotechnology, 2013. 31(6): p. 522-9.

28. Gyanchandani, R., et al., Intratumor Heterogeneity Affects Gene Expression Profile Test Prognostic Risk Stratification in Early Breast Cancer. Clinical cancer research : an official journal of the American Association for Cancer Research, 2016. 22(21): p. 5362-5369.

29. Sorensen, M., et al., Effect of intratumoral heterogeneity in oxygenation status on FMISO PET, autoradiography, and electrode PO2 measurements in murine tumors. International journal of radiation oncology, biology, physics, 2005. 62(3): p. 854-61.

30. Zhong, H., et al., Overexpression of hypoxia-inducible factor 1alpha in common human cancers and their metastases. Cancer research, 1999. 59(22): p. 5830-5.

31. Talks, K.L., et al., The expression and distribution of the hypoxia-inducible factors HIF-1alpha and HIF2alpha in normal human tissues, cancers, and tumor-associated macrophages. The American journal of pathology, 2000. 157(2): p. 411-21.

32. Zhang, W., et al., HIF-1alpha Promotes Epithelial-Mesenchymal Transition and Metastasis through Direct Regulation of ZEB1 in Colorectal Cancer. PloS one, 2015. 10(6): p. e0129603.

33. Lu, X. and Y. Kang, Hypoxia and hypoxia-inducible factors: master regulators of metastasis. Clinical cancer research : an official journal of the American Association for Cancer Research, 2010. 16(24): p. 5928-35.

34. Harris, A.L., Hypoxia--a key regulatory factor in tumour growth. Nature reviews. Cancer, 2002. 2(1): p. 38-47.

35. Wang, G.L., et al., Hypoxia-inducible factor 1 is a basic-helix-loop-helix-PAS heterodimer regulated by cellular $\mathrm{O} 2$ tension. Proceedings of the National Academy of Sciences of the United States of America, 1995. 92(12): p. 5510-4.

36. Chandel, N.S., et al., Reactive oxygen species generated at mitochondrial complex III stabilize hypoxiainducible factor-1alpha during hypoxia: a mechanism of $\mathrm{O} 2$ sensing. The Journal of biological chemistry, 2000. 275(33): p. 25130-8.

37. Brunelle, J.K., et al., Oxygen sensing requires mitochondrial ROS but not oxidative phosphorylation. Cell metabolism, 2005. 1(6): p. 409-14.

38. Semenza, G.L., Regulation of cancer cell metabolism by hypoxia-inducible factor 1. Seminars in cancer biology, 2009. 19(1): p. 12-6.

39. Semenza, G.L., Targeting HIF-1 for cancer therapy. Nature reviews. Cancer, 2003. 3(10): p. 721-32.

40. Ellinghaus, P., et al., BAY 87-2243, a highly potent and selective inhibitor of hypoxia-induced gene activation has antitumor activities by inhibition of mitochondrial complex I. Cancer Med, 2013. 2(5): p. 611-24.

41. Schockel, L., et al., Targeting mitochondrial complex I using BAY 87-2243 reduces melanoma tumor growth. Cancer Metab, 2015. 3: p. 11.

42. Vander Heiden, M.G., L.C. Cantley, and C.B. Thompson, Understanding the Warburg effect: the metabolic requirements of cell proliferation. Science, 2009. 324(5930): p. 1029-33.

43. Liberti, M.V. and J.W. Locasale, The Warburg Effect: How Does it Benefit Cancer Cells? Trends in biochemical sciences, 2016. 41(3): p. 211-8.

44. DiMauro, S. and M. Hirano, Merrf, in GeneReviews(R), R.A. Pagon, et al., Editors. 1993: Seattle (WA).

45. Wang, T.K., et al., Effects of carbonic anhydrase-related protein VIII on human cells harbouring an A8344G mitochondrial DNA mutation. The Biochemical journal, 2014. 459(1): p. 149-60. 
46. Wang, T.K., et al., Oncogenic roles of carbonic anhydrase 8 in human osteosarcoma cells. Tumour biology : the journal of the International Society for Oncodevelopmental Biology and Medicine, 2016. 37(6): p. 7989-8005.

47. Swietach, P., et al., The role of carbonic anhydrase 9 in regulating extracellular and intracellular ph in three-dimensional tumor cell growths. The Journal of biological chemistry, 2009. 284(30): p. 20299-310.

48. Rouschop, K.M., et al., PERK/elF2alpha signaling protects therapy resistant hypoxic cells through induction of glutathione synthesis and protection against ROS. Proceedings of the National Academy of Sciences of the United States of America, 2013. 110(12): p. 4622-7.

49. Ben Sahra, I., et al., Metformin, independent of AMPK, induces mTOR inhibition and cell-cycle arrest through REDD1. Cancer research, 2011. 71(13): p. 4366-72.

50. Koritzinsky, M., Metformin: A Novel Biological Modifier of Tumor Response to Radiation Therapy. International journal of radiation oncology, biology, physics, 2015. 93(2): p. 454-64.

51. Wouters, B.G. and M. Koritzinsky, Hypoxia signalling through mTOR and the unfolded protein response in cancer. Nature reviews. Cancer, 2008. 8(11): p. 851-64.

52. van der Harg, J.M., et al., The unfolded protein response mediates reversible tau phosphorylation induced by metabolic stress. Cell death \& disease, 2014. 5: p. e1393.

53. Urra, H., et al., Endoplasmic Reticulum Stress and the Hallmarks of Cancer. Trends in Cancer. 2(5): p. 252262.

54. Papa, L. and D. Germain, SirT3 regulates the mitochondrial unfolded protein response. Mol Cell Biol, 2014. 34(4): p. 699-710.

55. Liang, Q., et al., Bioenergetic and autophagic control by Sirt3 in response to nutrient deprivation in mouse embryonic fibroblasts. The Biochemical journal, 2013. 454(2): p. 249-57.

56. Hirschey, M.D., et al., SIRT3 regulates mitochondrial fatty-acid oxidation by reversible enzyme deacetylation. Nature, 2010. 464(7285): p. 121-5.

57. Fu, J., et al., trans-(-)-epsilon-Viniferin increases mitochondrial sirtuin 3 (SIRT3), activates AMP-activated protein kinase (AMPK), and protects cells in models of Huntington Disease. The Journal of biological chemistry, 2012. 287(29): p. 24460-72.

58. Qiu, X., et al., Calorie restriction reduces oxidative stress by SIRT3-mediated SOD2 activation. Cell Metab, 2010. 12(6): p. 662-7.

59. Qiao, A., et al., Sirt3-mediated mitophagy protects tumor cells against apoptosis under hypoxia. Oncotarget, 2016. 7(28): p. 43390-43400.

60. Bell, E.L., et al., SirT3 suppresses hypoxia inducible factor 1alpha and tumor growth by inhibiting mitochondrial ROS production. Oncogene, 2011. 30(26): p. 2986-96.

61. Finley, L.W., et al., SIRT3 opposes reprogramming of cancer cell metabolism through HIF1alpha destabilization. Cancer Cell, 2011. 19(3): p. 416-28.

62. Lim, J.H., et al., Sirtuin 1 modulates cellular responses to hypoxia by deacetylating hypoxia-inducible factor 1alpha. Mol Cell, 2010. 38(6): p. 864-78.

63. Zhong, L., et al., The histone deacetylase Sirt6 regulates glucose homeostasis via Hif1alpha. Cell, 2010. 140(2): p. 280-93. 



\section{Chapter}

\section{New approach of delivering cytotoxic drugs towards CAIX expressing cells: A concept of dual-target drugs}

Published in: European journal of medicinal chemistry, 2017. 127: p. 691-702. doi: 10.1016/j.ejmech.2016.10.037

Simon J.A. van Kuijk* Nanda Kumar Parvathaneni*, Raymon Niemans*, Marike W. van Gisbergen*, Fabrizio Carta, Daniela Vullo, Silvia Pastorekova, Ala Yaromina, Claudiu T. Supuran, Ludwig J. Dubois**, Jean-Yves Winum**, Philippe Lambin**

* contributed equally

** contributed equally 


\section{Highlights}

- New dual-target drugs combining cytotoxic agents with CAIX inhibitors were designed.

- Dual-targeting drugs may allow for specific drug delivery to hypoxic tumor areas.

- Increased binding affinity to CAIX was found for most of the dual-targeting drugs.

- Higher efficacy of dual-target ATR inhibitor was found in CAIX overexpressing cells.

- Design of alternative CAIX-targeting drugs may increase their therapeutic window.

\section{Abstract}

Carbonic anhydrase IX (CAIX) is a hypoxia-regulated and tumor-specific protein that maintains the $\mathrm{pH}$ balance of cells. Targeting CAIX might be a valuable approach for specific delivery of cytotoxic drugs, thereby reducing normal tissue side-effects. A series of dual-target compounds were designed and synthesized incorporating a sulfonamide, sulfamide, or sulfamate moiety combined with several different anti-cancer drugs, including the chemotherapeutic agents chlorambucil, tirapazamine, and temozolomide, two Ataxia Telangiectasia and Rad3-related protein inhibitors (ATRi), and the antidiabetic biguanide agent phenformin. An ATRi derivative (12) was the only compound to show a preferred efficacy in CAIX overexpressing cells versus cells without CAIX expression when combined with radiation. Its efficacy might however not solely depend on binding to CAIX, since all described compounds generally display low activity as carbonic anhydrase inhibitors. The hypothesis that dual-target compounds specifically target CAIX expressing tumor cells was therefore not confirmed. Even though dualtarget compounds remain an interesting approach, alternative options should also be investigated as novel treatment strategies.

\section{Extracellular space}

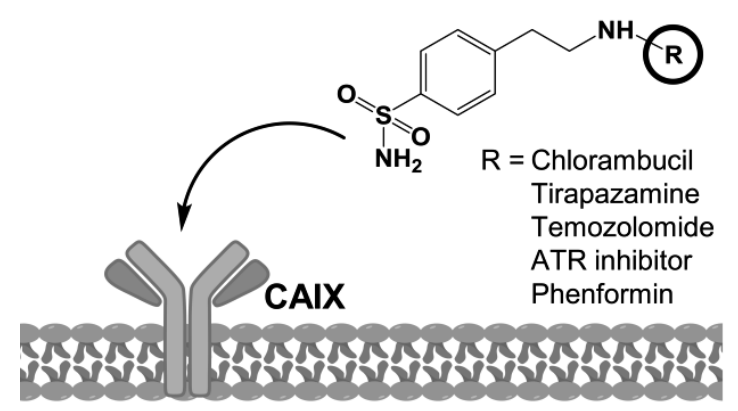

Intracellular space 


\section{Introduction}

Solid tumors are characterized by a hypoxic microenvironment caused by their immature and inadequate vascular supply of oxygen and nutrients. These hostile hypoxic conditions result in a phenotype that is associated with a worse prognosis [1] and resistance to standard treatment options such as radiotherapy, chemotherapy, and surgery [2-4]. Several different approaches are currently being investigated to target these hypoxic areas to make tumors more sensitive to standard treatment modalities [5-7].

Carbonic anhydrase IX (CAIX) can be a valuable therapeutic target since it plays an important role in maintaining the intracellular $\mathrm{pH}$ homeostasis [8, 9]. Furthermore its expression is predominantly tumor specific $[5,8,10]$ and directly regulated via the hypoxia-inducible factor (HIF) pathway [11]. Even though alternative pathways are also able to modulate CAIX expression [12-14], its significant prognostic value in many different tumor types [15] has promoted investigations in its use as an imaging agent for diagnostic and prognostic purposes [5, 16-19]. Together these characteristics of CAIX support investigations into the therapeutic targeting of CAIX to improve efficacy of standard treatments. The function of CAIX is evolutionary conserved and catalyzes the hydration of carbon dioxide to bicarbonate at the cell membrane. The bicarbonate is transported back intracellular from the extracellular space, whereas the free proton is extruded in the extracellular space. CAIX thereby maintains the balance between an acidic extracellular and alkaline intracellular $\mathrm{pH}$ of tumor cells, the latter of which would otherwise acidify due to the increased acid production resulting from their glycolytic metabolism [8, 9]. Many different inhibitors are currently being developed to specifically target the tumor-associated CAIX isoform and have shown promise in reducing tumor cell survival, migration, invasion, and reduce tumor xenograft growth and metastases formation [20-23]. Furthermore, the combination therapy of CAIX inhibitors (CAIXi) with standard treatment options was previously found to increase the efficacy of radiotherapy [24] and of weakly basic chemotherapeutic agents such as doxorubicin [25].

The predominant expression of CAIX on hypoxic tumor cells can also be exploited to direct cytotoxic agents specifically to those CAIX expressing cancer cells thereby possibly minimizing normal tissue toxicity. This can be achieved by conjugating anticancer drugs with CAIX inhibiting molecules that bind to the $\mathrm{Zn}^{2+}$ active site of CAIX and hence inhibit its enzymatic function [8, 26, 27], i.e. a so-called dual-targeting approach. Our group showed previously that such a dual-target approach with a sulfamide CAIXi moiety coupled to the radiosensitizing compound nitroimidazole to be a more effective radiosensitizer than an indanesulfonamide CAIXi [28]. Alternative novel dual-target compounds have been developed to investigate this strategy of dual-targeting further in the context of anti-cancer agents to target CAIX. Here we have designed five different classes of dual-target compounds conjugated to CAIXi (sulfonamide, sulfamide, or 
sulfamate), which included the chemotherapeutic anti-cancer agents chlorambucil, tirapazamine, and temozolomide, two ataxia telangiectasia and Rad3-related protein inhibitors (ATRi), and the biguanide agent phenformin, previously used in diabetes treatment. We hypothesize that these new dual-target compounds will have the ability to specifically target CAIX expressing cells and modulate their efficacy in a CAIXdependent manner.

\section{Results and discussion}

\section{Chemistry}

Chlorambucil was converted to its acid chloride [29] 1 by using oxalyl chloride. This chlorambucil acid chloride reacted with different benzene sulfonamides under basic condition to obtain good yields of chlorambucil derivatives $2 \mathrm{a}, 2 \mathrm{~b}$ and $2 \mathrm{c}$. Chlorambucil carbamate derivatives were obtained by converting compound 1 into a methyl ester [30] using methanol. This ester was reduced to alcohol [31], i.e. compound 3, after treating with lithium aluminium hydride. Compound 3 was treated with triphosgene to obtain its respective chloroformate [32], i.e. compound 4 (Scheme 2). The reaction of chlorambucil chloroformate (4) with different benzene sulfonamides resulted in compounds 5a, 5b and $5 c$ (Scheme 3).

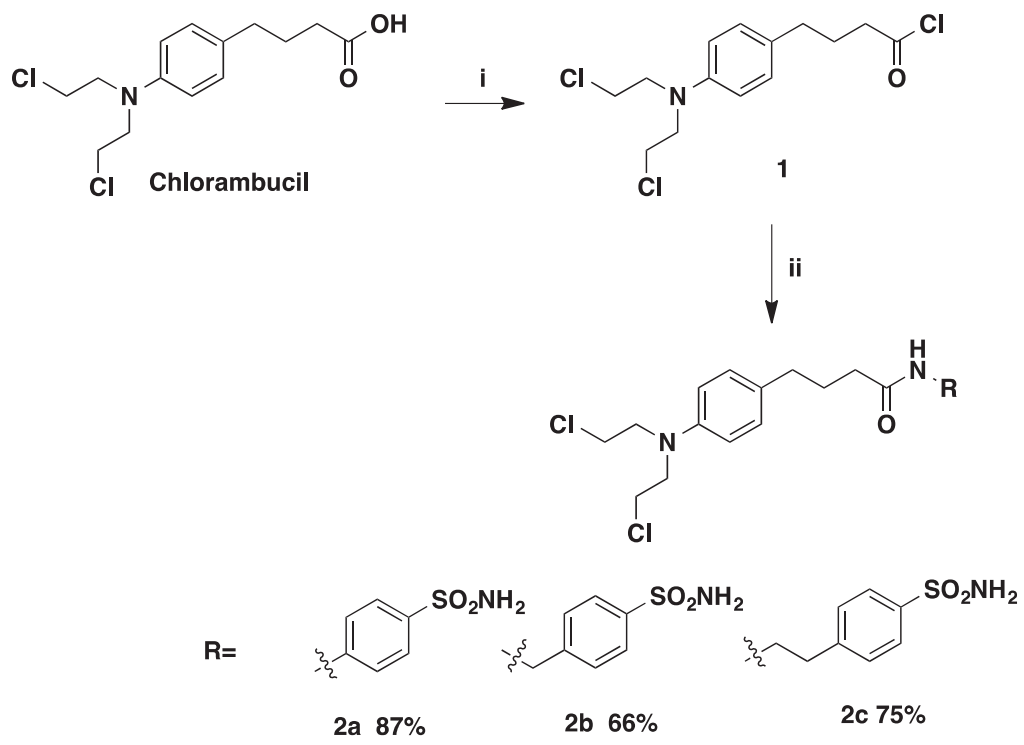

Scheme 1. Reagents and conditions: (i) $(\mathrm{COCl})_{2}$, DMF, DCM, $0^{\circ} \mathrm{C}-\mathrm{rt}$; (ii) DIPEA, THF, $0^{\circ} \mathrm{C}-\mathrm{rt}$. 


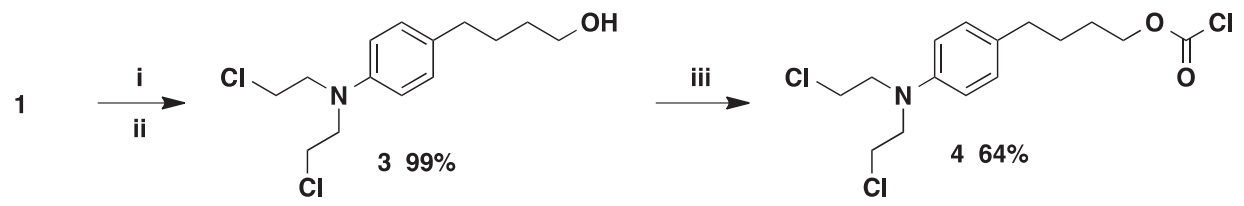

Scheme 2. Reagents and conditions: (i) MeOH, DCM; (ii) LAH, THF; (iii) Triphosgene, $\mathrm{Na}_{2} \mathrm{CO}_{3}$, Toluene, DMF.<smiles>[R]NC(=O)OCCCCc1ccc(N(CCCl)CCCl)cc1</smiles>

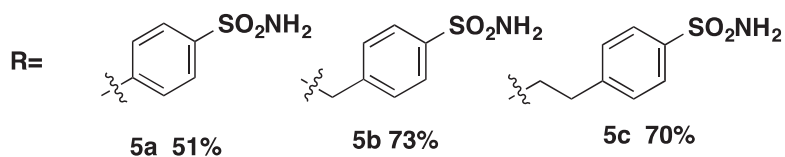

Scheme 3. Reagents and conditions: (i) DIPEA, THF, $0{ }^{\circ} \mathrm{C}-\mathrm{rt}$.

Tirapazamine derivatives 8 and 11 were synthesized from 6 and 9 with the previously described procedure [33]. In short, 6 and 9 reacted with 4-(2-aminoethyl) benzene sulfonamide under reflux conditions and was followed by oxidation of the mono-oxides (Scheme 4).

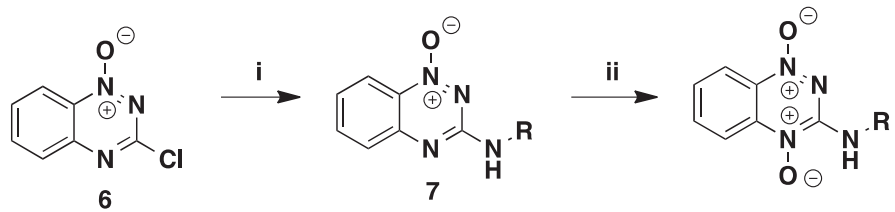

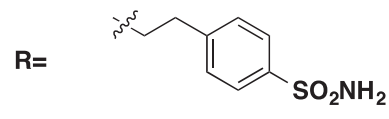

$896 \%$<smiles>[2H]Nc1nc2cc3c(cc2[n+]([O-])n1)CCC3CC(C)C</smiles>

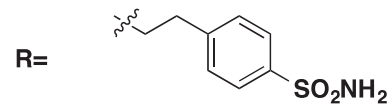

$1146 \%$

Scheme 4. Reagents and conditions: (i) $\mathrm{RNH}_{2}$ (3 equiv.), DME, reflux; (ii) TFAA, $\mathrm{H}_{2} \mathrm{O}_{2}, \mathrm{DCM}$, rt. 
The ATRi derivatives 12 and 13 were synthesized from commercially available VE-821 and VE-822 (MedChemTronica) using a classical synthetic strategy described previously [25] (Scheme 5).<smiles>CS(=O)(=O)c1ccc(-c2cnc(N)c(C(=O)Nc3ccccc3)n2)cc1</smiles>

VE 821<smiles>CNCc1ccc(-c2cc(-c3nc(-c4ccc(S(=O)(=O)C(C)C)cc4)cnc3N)on2)cc1</smiles><smiles>CS(=O)(=O)c1ccc(-c2cnc(NS(=O)(=O)O)c(C(=O)Nc3ccccc3)n2)cc1</smiles>

$1258 \%$<smiles>CNCc1ccc(-c2cc(-c3nc(-c4ccc(S(=O)(=O)C(C)C)cc4)cnc3NS(N)(=O)=O)on2)cc1</smiles>

Scheme 5. Reagents and conditions: (i) $\mathrm{ClSO}_{2} \mathrm{NCO}, \mathrm{tBuOH}, \mathrm{NEt}_{3}, \mathrm{DCM}, 0{ }^{\circ} \mathrm{C}$ to rt; (ii) $20 \% \mathrm{TFA}-\mathrm{DCM}$.

Commercially purchased temozolomide (SelleckChem) was converted into its respective acid by treating with concentrated sulfuric acid and sodium nitrate at $0{ }^{\circ} \mathrm{C}-15{ }^{\circ} \mathrm{C}$ (Scheme 6) [34]. Reacting the temozolomic acid with different benzenesulfonamides, aminoxysulfonamide [35] and 5-amino-1, 3,4-thiadiazole-2-sulfonamide hydrochloride under known amide bond formation conditions [34] resulted in compounds 15a, 15b, $15 c$ and $15 d$ (Scheme 7).<smiles>Cn1nnc2c(C(N)=O)ncn2c1=O</smiles>

TMZ

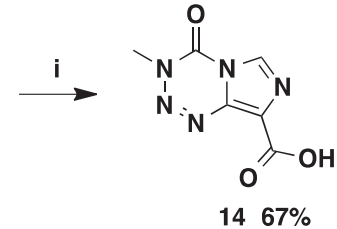

$1467 \%$

Scheme 6. Reagents and conditions: (i) Con. $\mathrm{H}_{2} \mathrm{SO}_{4}, \mathrm{NaNO}_{2}, \mathrm{O}^{\circ} \mathrm{C}$ to $15^{\circ} \mathrm{C}$ 
<smiles>[R]NC(=O)c1ncn2c(=O)n(C)nnc12</smiles>

$\mathbf{R}=$<smiles>CCCc1ccc(S(N)(=O)=O)cc1</smiles>

15 a $50 \%$

\section{?. $\mathrm{O} \cdot \mathrm{SO}_{2} \mathrm{NH}_{2}$}

15b $41 \%$<smiles>CCc1ccc(S(=O)(=O)N[Na])cc1</smiles>

15 c $88 \%$<smiles>Cc1nnc(S(=O)(=O)O[Na])s1</smiles>

$15 d 95 \%$

Scheme 7. Reagents and conditions: (i) BOP, $\mathrm{NEt}_{3,}$ DCM, rt.

The compound 18 was obtained by a slight modification based on the method reported by Kelarev et al. [36]. The commercially available compounds 4-(2-aminoethyl) benzenesulfonamide 16 and cyanoguanidine 17 were coupled in n-butanol using a stoichiometric amount of hydrochloric acid (Scheme 8).<smiles>NCCc1ccc(S(=O)(=O)O)cc1</smiles>

16<smiles>N#CNC(=N)N</smiles>

17

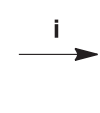

$\mathrm{H}_{2} \mathrm{NO}_{2} \mathrm{~S}$<smiles>Cc1ccc(CCNC(=N)NC(=N)N)cc1</smiles>

18

Scheme 8. Reagents and conditions: (i) $6.0 \mathrm{M} \mathrm{HCl} \mathrm{aq,} \mathrm{nBuOH,} \mathrm{reflux.}$

\section{Binding affinity human CAs}

Increased binding affinity to human carbonic anhydrases (CAs) as compared with their respective parental compound are observed (Table 1) for most of the compounds, except for the CAIXi conjugated ATRi (12 and 13), which do not bind to any of the four tested human CA isoforms included (Ki> $50000 \mathrm{nM})$. The Ki values of the other dualtargeting compounds are higher than of the previously reported CAIXi [20] with 15a showing relatively good $\mathrm{Ki}$ for the CAII and CAIX isoforms, but not for CAXII. Only the phenformin derivative 18 was found to be selective for the transmembrane CAIX and CAXII isoforms. To investigate whether the biological efficacy of the functionalized compounds is dependent on CAIX expression, canine kidney epithelial (MDCK) cells without CAIX (CAIX), i.e. both human and canine [37], or MDCK cells transfected with human CAIX [37], i.e. overexpressing CAIX $\left(\mathrm{CAIX}^{+}\right)$, were used. Western blotting 
confirmed differential expression of CAIX in these cells both under normoxic and hypoxic conditions (Supplementary Fig. S1).

Table 1: Binding affinity $\left(K_{\mathrm{i}}\right)$ to human CAI, CAII, CAIX, and CAXII of the parental compounds (bold) and their CAIXi conjugated derivatives.

\begin{tabular}{|c|c|c|c|c|c|c|}
\hline \multirow[b]{2}{*}{ Compound } & \multicolumn{4}{|l|}{$K_{\mathrm{i}}(\mathrm{nM})^{a}$} & \multicolumn{2}{|l|}{ Selectivity Ratios ${ }^{b}$} \\
\hline & hCA I & hCA II & hCA IX & hCA XII & $K_{\mathrm{i}} \mathrm{hCA} \| / K_{\mathrm{i}} \mathrm{hCA}$ IX & $K_{\mathrm{i}} \mathrm{hCA} \| / K_{\mathrm{i}} \mathrm{hCA}$ XII \\
\hline Chlorambucil & $>50000$ & $>50000$ & $>50000$ & $>50000$ & & \\
\hline $2 a$ & 73.0 & 9.0 & 172 & 689 & 0.05 & 0.01 \\
\hline $2 b$ & 5950 & 747 & 8970 & 7340 & 0.08 & 0.10 \\
\hline $2 c$ & 8400 & 450 & 4610 & 10160 & 0.10 & 0.04 \\
\hline $5 a$ & 5580 & 553 & 2740 & 9380 & 0.20 & 0.06 \\
\hline $5 c$ & 6140 & 265 & 4130 & 9570 & 0.06 & 0.03 \\
\hline $5 c$ & 5670 & 504 & 3850 & 13600 & 0.13 & 0.04 \\
\hline Tirapazamine & $>50000$ & $>50000$ & $>50000$ & $>50000$ & & \\
\hline 8 & 567 & 7.1 & 383 & 14600 & 0.02 & $<0.01$ \\
\hline 11 & 428 & 8.1 & 307 & 624 & 0.03 & 0.01 \\
\hline Temozolomide & $>50000$ & $>50000$ & $>50000$ & $>50000$ & & \\
\hline $15 a$ & 91.3 & 9.2 & 37.1 & 9300 & 0.25 & $<0.01$ \\
\hline $15 b$ & $>50000$ & $>50000$ & $>50000$ & $>50000$ & & \\
\hline $15 c$ & 539 & 90.5 & 271 & 12400 & 0.33 & 0.01 \\
\hline $15 d$ & 743 & 15.7 & 176 & 92.7 & 0.09 & 0.17 \\
\hline ATRi VE-821 & $>50000$ & $>50000$ & $>50000$ & $>50000$ & & \\
\hline 12 & $>50000$ & $>50000$ & $>50000$ & $>50000$ & & \\
\hline ATRi VE-822 & $>50000$ & $>50000$ & $>50000$ & $>50000$ & & \\
\hline 13 & $>50000$ & $>50000$ & $>50000$ & $>50000$ & & \\
\hline Phenformin & $>50000$ & $>50000$ & $>50000$ & $>50000$ & & \\
\hline 18 & 4435 & 501 & 20.2 & 1.7 & 24.8 & 295 \\
\hline Acetazolamide $^{c}$ & 250 & 12.1 & 25.3 & 5.7 & 0.48 & 2.12 \\
\hline
\end{tabular}

${ }^{a}$ Values reported (in $\mathrm{nM}$ ) are the average of three different estimations with errors between $5-10 \%$ of the reported values. Reported values $>50000$ indicates no binding of the compound towards the CA isoforms.

${ }^{b}$ Selectivity ratios of the cytosolic hCAll over the tumor-associated hCAIX and hCAXII isoforms.

${ }^{c}$ Non-specific CAi acetazolamide is included as a reference.

\section{Chlorambucil derivatives}

Chlorambucil (4-[p-[bis(2-chloroethyl)amino]phenyl]butyric acid) is a nitrogen mustard that acts as a bifunctional alkylating agent used for decades to treat cancers originating in the blood and lymphatic system, e.g. chronic lymphocytic leukemia and lymphomas [38]. Even though reported data suggest chlorambucil efficacy to increase in an acidic microenvironment [39], the CAIXi moiety (benzenesulfonamides) with different linkers (i.e. amide, carbamate) might allow for specific targeting of the compounds to these areas in the tumor. The six CAIXi conjugated chlorambucil derivatives (2a, 2b, 2c, 5a, 5b, and $5 c$, Scheme 1 and Scheme 2) lead to reduced cell viability as compared to the parental compound, which was only marginally effective (Table 2, Supplementary Fig. 
S1). The therapeutic efficacy of these compounds however was not increased in the CAIX ${ }^{+}$MDCK cells as compared with the CAIX MDCK cells. Furthermore, none of the six compounds showed an increased efficacy upon hypoxia exposure $\left(0.2 \% \mathrm{O}_{2}\right)$. In contrast, some of the chlorambucil dual-target derivatives were less cytotoxic (i.e. higher $I C_{50}$, Table 2) in CAIX expressing cells independent of oxygen levels, which contradicts the studies demonstrating an increased efficacy of chlorambucil in an acidic micromilieu [39]. All together from these results it can be concluded that the CAIXi conjugated chlorambucil derivatives do not show an increased efficacy in a CAIX or hypoxia dependent manner.

Table 2. Estimated $I C_{50}$ of the cytotoxic parental compounds (bold) and their CAIXi conjugated derivatives obtained with cell viability assays for MDCK CAIX and MDCK CAIX ${ }^{+}$cells exposed to normoxic and hypoxic conditions.

\begin{tabular}{|c|c|c|c|c|}
\hline \multirow[b]{2}{*}{ Compound } & \multicolumn{2}{|c|}{ Normoxia $(\mu \mathrm{M})^{a}$} & \multicolumn{2}{|c|}{ 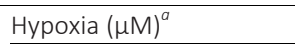 } \\
\hline & $\overline{\text { CAIX }}$ & CAIX $^{+}$ & $\overline{\text { CAIX }}$ & CAIX $^{+}$ \\
\hline Chlorambucil $^{b}$ & 93 & $>100$ & $\sim 100$ & $>100$ \\
\hline $2 a$ & $\sim 100$ & 87 & $\sim 100$ & 95 \\
\hline $2 b$ & 18 & 98 & 14 & 92 \\
\hline $2 c$ & 18 & $\sim 100$ & 18 & $\sim 100$ \\
\hline $5 a$ & 8 & 56 & 8 & 62 \\
\hline $5 b$ & 86 & 98 & 52 & 100 \\
\hline $5 c$ & 89 & 99 & 81 & $\sim 100$ \\
\hline Tirapazamine $^{c}$ & $>300$ & $>300$ & $<50$ & 95 \\
\hline 8 & $>300$ & $>300$ & $\sim 300$ & $>300$ \\
\hline 11 & $>300$ & $>300$ & $>300$ & $>300$ \\
\hline Temozolomide $^{d}$ & 775 & $>1000$ & $\sim 1000$ & $>1000$ \\
\hline $15 a$ & $>1000$ & $>1000$ & $>1000$ & $>1000$ \\
\hline $15 b$ & 719 & $>1000$ & $\sim 1000$ & $>1000$ \\
\hline $15 c$ & $>1000$ & $>1000$ & $>1000$ & $>1000$ \\
\hline $15 d$ & $>1000$ & $>1000$ & $>1000$ & $>1000$ \\
\hline
\end{tabular}

${ }^{a}$ No $I C_{50}$ reached is indicated with $>$. Estimated $I C_{50}$ value higher than the maximum concentration included is indicated with .

${ }^{b}$ Included concentrations for chlorambucil were 1,10 , and $100 \mu \mathrm{M}$.

'Included concentrations for tirapazamine were 50, 100, 200, and $300 \mu \mathrm{M}$.

${ }^{d}$ Included concentrations for temozolomide were 100, 400, 700, and $1000 \mu \mathrm{M}$.

\section{Tirapazamine derivatives}

The hypoxia-activated prodrug tirapazamine (3-amino-1,2,4-benzotriazine-1,4-dioxide) has been tested in several clinical trials in combination with chemo- and/or radiotherapy [40]. The cytotoxicity of tirapazamine results from activation by reductive enzymes that add an electron to the parent drug to produce a radical species that causes DNA damage. Nevertheless, no definitive conclusions regarding its clinical efficacy can be drawn since addition of tirapazamine to standard treatment (i.e. radiochemotherapy) did not result in an increased benefit in phase III clinical trials. In 
addition, tirapazamine treatment was often characterized by toxic side-effects, such as nausea, vomiting, and diarrhea, which limited its therapeutic gain [40]. Targeting tirapazamine towards the CAIX expressing (hypoxic) areas in tumors by conjugating tirapazamine with the benzenesulfonamide CAIXi might thereby prove a valuable approach to reduce normal tissue toxicity and increase the efficacy of the compounds (8 and 11) in CAIX expressing (hypoxic) cells. Cell viability assays (Table 2, Supplementary Fig. S2) confirmed that the parental compound was specifically effective in hypoxic cells, and more effective in CAIX cells (IC $\mathrm{C}_{50}<50$ versus $\left.95 \mu \mathrm{M}, \mathrm{p}=0.037\right)$. The CAIXi conjugated tirapazamine derivatives however abrogated the effect observed for the parental compound, both during hypoxia and normoxia, which was independent of CAIX levels (Table 2).

\section{Temozolomide derivatives}

The current treatment of glioblastoma is based on radiotherapy combined with temozolomide, which has been shown to increase survival in phase III clinical trials [41]. Temozolomide is a methylating agent that spontaneously hydrolyzes to its active metabolite 3-methyl-(triazen-1-yl)imidazole-4-carboxamide (MTIC) at physiological pH [42]. The acidic extracellular $\mathrm{pH}$ in tumors might therefore reduce spontaneous temozolomide conversion and thereby decrease its efficacy. Inhibiting CAIX function is known to decrease extracellular acidification in vitro $[24,25,28,43]$, and we hypothesized that conjugating temozolomide with a sulfonamide or sulfamate moiety (15a, 15b, 15c, and 15d) will specifically target hypoxic tumors and increase temozolomide conversion and thereby its efficacy. Nevertheless, while temozolomide resulted in lower cell viability in CAIX- cells, consistent with the $\mathrm{pH}$-dependent mechanism of activation, the CAIXi conjugated temozolomide derivatives $15 \mathrm{a}, 15 \mathrm{c}$, and $15 \mathrm{~d}$ were ineffective in reducing cell viability in both MDCK cell lines during normoxic and hypoxic conditions within the concentration range tested in the present study (Table 2, Supplementary Fig. S3). In contrast, 15b was similarly effective as the parental temozolomide compound (Table 2).

This dual-target compound was therefore investigated further in clonogenic survival assays in which the medium of the cells was acidified because of CAIX function during hypoxic conditions (Supplementary Fig. S1)[44]. Temozolomide was again more effective in reducing clonogenic cell survival in the CAIX MDCK cells as compared with the CAIX ${ }^{+}$MDCK cells (Fig. 1), similarly to its efficacy on cell viability. During hypoxia however temozolomide caused no difference in clonogenic survival as compared to normoxia, even though hypoxia is required to activate CAIX and cause extracellular acidification $[43,45]$ and is therefore hypothesized to reduce temozolomide conversion and efficacy. In contrast, the CAIXi conjugated derivative $15 \mathrm{~b}$ significantly reduced clonogenic cell survival in hypoxic versus normoxic conditions in the $\mathrm{CAIX}^{+}$cells 
(surviving fraction is $46.1 \pm 3.1$ versus $26.1 \% \pm 7.9$ during normoxia and hypoxia respectively, $p<0.05$ ). Nevertheless, the effect of $15 \mathrm{~b}$ on survival was not significantly different from the parental temozolomide compound. In addition, the low binding affinity of the compound (Table 1 ) combined with its relatively low efficacy in the CAIX ${ }^{+}$ as compared to the CAIX $X^{-}$cells minimizes its potential for further development. These results furthermore suggest that temozolomide efficacy is not affected by CAIX dependent changes in extracellular $\mathrm{pH}$ during hypoxia. A reduction of temozolomide conversion and efficacy might require lower $\mathrm{pH}$ levels, i.e. $\mathrm{pH}<6.6$, which may have not been achieved in the present experiments $[8,9,39]$.

\section{A Normoxia}

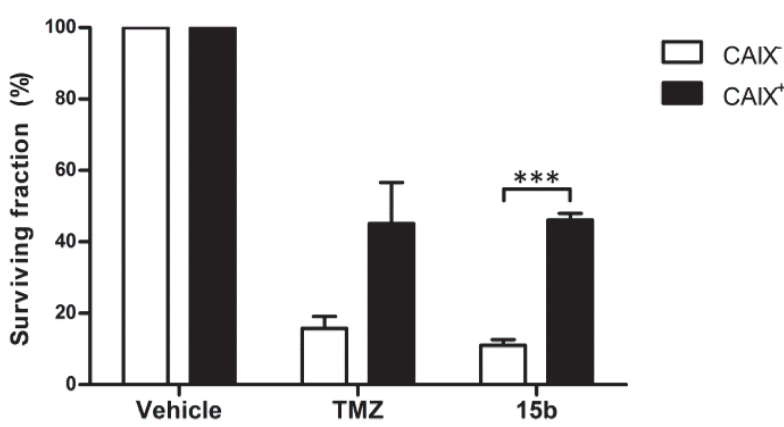

B

Hypoxia

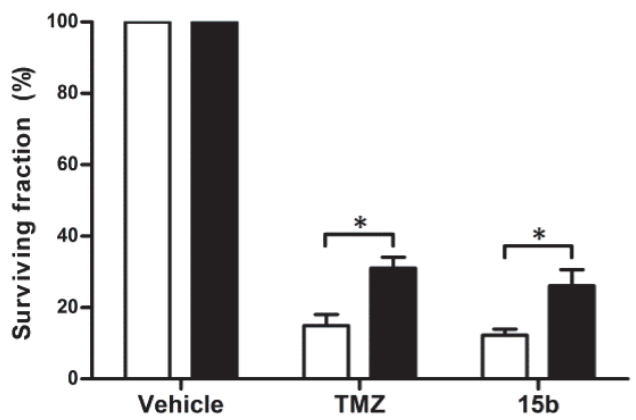

Figure 1. Clonogenic cell survival of confluent MDCK CAIX and CAIX ${ }^{+}$cells during normoxia $\left(21 \% \mathrm{O}_{2}\right)$ and hypoxia $\left(0.02 \% \mathrm{O}_{2}\right)$ when exposed to temozolomide (TMZ) and the CAIXi conjugated derivative 15b. Surviving fraction (\%) was normalized to vehicle control. Average \pm SEM of three independent biological repeats is shown. Asterisks indicate statistical significance $\left({ }^{*} p<0.05 ;{ }^{* *} p<0.001\right)$.

\section{ATR inhibitor derivatives}

Preclinical experiments have shown that Ataxia Telangiectasia and Rad3-related protein inhibitors (ATRi) reduce the DNA repair capacity resulting in enhanced cell death and decreased tumor growth when combined with either chemo- or radiotherapy [46-48]. 
However, ATRi are not highly tumor specific, thus targeting these compounds towards the CAIX expressing areas of a tumor might increase their therapeutic benefit. The effect on cell viability of the parental ATRi (VE-821 and VE-822) and their CAIXi conjugated derivatives (12 and 13) was tested in combination with radiotherapy to induce DNA damage where a higher radiation dose was applied to anoxic cells, since those are more radioresistant $[49,50]$. The parental ATRi and the CAIXi conjugated derivatives in combination with radiation decreased cell viability as compared to radiation only in the CAIX ${ }^{+}$cells $(p<0.05)$ under both normoxic and anoxic conditions, but not in the CAIX ${ }^{-}$cells (Fig. 2). The only exception is the derivative 13, which had no significant effect on cell viability during anoxic conditions in both cell lines as compared to radiation alone $\left(p=0.09\right.$ and $p=0.08$ for $\mathrm{CAIX}^{-}$and $\mathrm{CAIX}^{+}$cells, respectively). More importantly, the CAIXi conjugated derivative 12 was more effective than its respective parental ATRi (VE-821) in the CAIX ${ }^{+}(p<0.01$ during normoxia and anoxia), but not the CAIX cells ( $p=0.52$ and $p=0.72$ for normoxia and anoxia, respectively), suggesting a CAIX specific effect. In contrast, the CAIXi conjugated derivative 13 in combination with radiation was less effective in reducing cell viability than the parental compound VE-822 in CAIX ${ }^{+}$cells ( $p<0.001$ and $p<0.01$ during normoxic and anoxic conditions, respectively). Although radiation induced similar effects on cell viability during normoxic and anoxic conditions, the efficacy of derivative 12 did not increase further during anoxic conditions as compared to normoxic conditions, even though CAIX expression is upregulated under hypoxic conditions (Supplementary Fig. S1) and these conditions are essential for CAIXi binding $[43,45]$. Although derivative 12 indeed proved to be more effective in $\mathrm{CAIX}^{+}$than in CAIX cells in combination with radiation, its efficacy might however not be solely dependent on binding to CAIX, which is consistent with unfavorable $K i$ values of the compound (Table 1 ). Exposing both cell lines to ATRi without irradiation decreased cell viability of both cell lines during normoxic and anoxic conditions, although this effect appeared to be slightly more pronounced in the CAIX ${ }^{-}$ cells (Supplementary Fig. S5). Differences in ATRi response between the cell lines when combined with radiation might be explained by a lower number of cells in the resistant S-phase of the cell cycle [51], or by a decreased DNA repair capacity in cells with lower intracellular $\mathrm{pH}$ [52-54], i.e. those that do not express CAIX. This may also explain the difference in sensitivity to cytotoxic drugs between both cell lines, although further investigations are required to prove this causal relationship. 

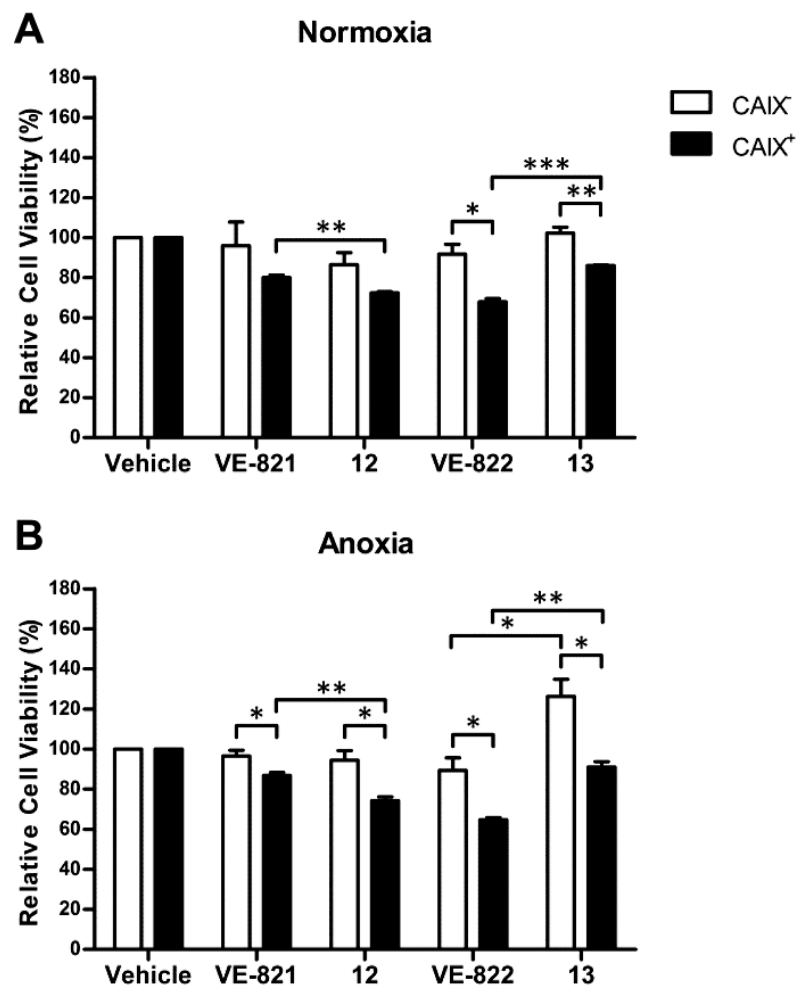

Figure 2. Relative cell viability (\%) in MDCK CAIX and CAIX ${ }^{+}$cells exposed to ATR inhibitors (VE-821 and VE822 ) or the CAIXi conjugated derivatives (12 and 13) in combination with radiation during normoxia $\left(21 \% \mathrm{O}_{2}\right)$ and anoxia $\left(<0.02 \% \mathrm{O}_{2}\right)$. Normoxic cells were irradiated with $2 \mathrm{~Gy}$ and anoxic cells with $4 \mathrm{~Gy}$ to induce similar effects on cell viability. Cells were exposed to 500 nM VE-821 and 12, and to 50 nM VE-822 and 13. Average \pm SEM of three independent biological repeats is shown. Asterisks indicate statistical significance $\left({ }^{*} p<0.05\right.$; $* * p<0.01 ; * * * p<0.001)$.

\section{Phenformin derivatives}

Phenformin (1-(diaminomethylidene)-2-(2-phenylethyl)guanidine) is a drug used to treat diabetes, but was withdrawn from the North-American market in the 1970s by the Food and Drug Administration (FDA) due to a high risk of developing lactic acidosis [55]. Treating patients with a similar but less potent drug metformin was found to be associated with a decrease in cancer incidence and an increased life span of cancer patients [56]. The repurposing of these compounds as anti-cancer agents is therefore being investigated where phenformin is found to be more lipophilic, thereby requiring less active transport than metformin [57]. The proposed mechanism of action of phenformin is its ability to inhibit mitochondrial respiration, which will consequently result in a decreased ATP production, thereby reducing tumor cell growth and 
improving tumor oxygenation as a result of decreased oxygen consumption $[58,59]$. Conjugating phenformin with CAIXi might make the drug more tumor-specific leading to reduced normal tissue toxicity. Since tumor cells are more sensitive to phenformin treatment due to their altered energy metabolism, human colorectal HCT116 cells, with or without CAIX knockdown $[24,28]$ were used to study the effect of phenformin and its CAIXi conjugated derivative 18 on mitochondrial respiration. Western blotting confirms Iow expression of CAIX in CAIX KD cells under hypoxic conditions as compared with control cells (Supplementary Fig. S1B). As expected, CAIX levels were low in both cell lines under normoxic conditions. Phenformin significantly reduced Oxygen Consumption Rate $(O C R)$ in both cell lines $(p<0.05)$, independent of CAIX expression levels (Fig. 3). In contrast, the CAIXi conjugated derivative 18 was ineffective in reducing OCR, even when a fourfold higher concentration was used.

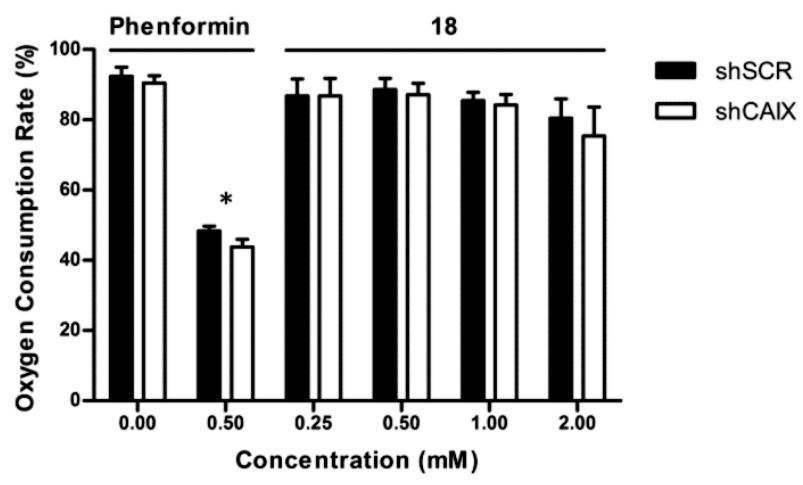

Figure 3. Oxygen consumption rate (OCR) of HCT116 cells with CAIX (shSCR) or without CAIX expression (shCAIX) exposed to phenformin or the CAIXi conjugated derivative 18. OCR was normalized to baseline OCR levels before compound injection. Average \pm SEM of four independent biological repeats is shown. Asterisks indicate statistical significance $(* p<0.05)$.

\section{Conclusion}

Overall our hypothesis that newly designed dual-target drugs are more selective for CAIX expressing cells and are able to modulate their own efficacy by inhibiting CAIX function was not confirmed. Of all derivatives included, only one (i.e. the ATRi derivative 12) proved more effective than its parental compound when combined with irradiation in CAIX ${ }^{+}$cells versus CAIX ${ }^{-}$cells. Nevertheless, the effect of this compound may not only be related to binding of the compound to CAIX due to limited binding affinity and the lack of further increase in its efficacy under hypoxic conditions. The rest of the derivatives included in this study did not show an increased efficacy in CAIX ${ }^{+}$versus CAIX cells, or an efficacy that depended on oxygen levels, i.e. hypoxia versus normoxia. 
Nevertheless, since the parental compounds proved effective in these experiments the conjugation of the CAIXi moiety with the cytotoxic compounds may have caused conformational changes, thereby altering the compounds efficacy. In addition, these conformational changes may have also limited the binding of the CAIXi moiety (sulfonamide, sulfamide or aminoxysulfonamide) into the $\mathrm{Zn}^{2+}$ containing active pocket of CAIX, which explains the lack of CAIX specificity and binding affinity for human CAs (Table 1) of the compounds. Alternative strategies to target the CAIX expressing cells in a tumor, e.g. antibody targeting or an increased number of CAIXi conjugated molecules [60], might therefore be more promising options to pursue in the future.

\section{Experimental section}

\section{Chemistry}

General

Unless otherwise specified, reagents and solvents were of commercial quality and were used without further purification. All reactions were carried out under an inert atmosphere of nitrogen. TLC analyses were performed on silica gel $60 \mathrm{~F}_{254}$ plates (Merck Art.1.05554). Spots were visualized under $254 \mathrm{~nm}$ UV illumination, or by ninhydrin solution spraying. Melting points ( $\mathrm{mp}$ ) were determined on a Büchi Melting Point 510 and are uncorrected. ${ }^{1} \mathrm{H}$ and ${ }^{13} \mathrm{C}$ NMR spectra were recorded on Bruker DRX-400 spectrometer using DMSO-d6 as a solvent and tetramethylsilane as an internal standard. For ${ }^{1} \mathrm{H}$ NMR spectra, chemical shifts are expressed in $\delta$ (ppm) downfield from tetramethylsilane, and coupling constants $(J)$ are expressed in Hertz. Electron Ionization mass spectra were recorded in positive or negative mode on a Waters MicroMass ZQ. All compounds that were tested in the biological assays were analyzed by Highresolution ESI mass spectra (HRMS) using on a Q-ToF I mass spectrometer fitted with an electrospray ion source in order to confirm the purity of $>95 \%$.

\section{4-(4-(Bis(2-chloroethyl)amino)phenyl)butanoyl chloride (1)}

Oxalyl chloride (32.8 mmol, 2.0 equiv.) was added slowly over a period of $1.0 \mathrm{~h}$ at 5-10 ${ }^{\circ} \mathrm{C}$ to a stirred solution of chlorambucil ( $16.4 \mathrm{mmol}, 1.0$ equiv.) in DCM (25.0 mL, 5.0 vol) and a catalytic amount of $\mathrm{N}, \mathrm{N}$-dimethylformamide. The reaction mixture was stirred at ambient temperature for 2-3 h, after which excess oxalyl chloride and DCM were removed under reduced pressure. The chlorambucil acid chloride that was obtained was a pale green solid in quantitative yield, which was used as such for the synthesis of Compounds $2 \mathrm{a}-\mathrm{c}$. 
4-(4-(Bis(2-chloroethyl)amino)phenyl)butan-1-ol (3)

Compound 1 (15.5 mmol, 1.0 equiv.) was dissolved in DCM (125 mL) and methanol (75 $\mathrm{mL}, 3 \mathrm{vol}$ ) was slowly added over a period of $1 \mathrm{~h}$ at $15-20^{\circ} \mathrm{C}$. The reaction mixture was stirred at ambient temperature for $2 \mathrm{~h}$. The reaction mixture was concentrated and the residue was dissolved in ethyl acetate $(125 \mathrm{~mL}, 5 \mathrm{vol})$ and washed successively with a $5 \%$ aq. $\mathrm{NaHCO}_{3}$. Evaporation of the solvent under reduced pressure resulted in the chlorambucil methyl ester (16.4 mmol, 1.0 equiv.) in 95\% yield as a light brown oil, which was added to a suspension of lithium aluminium hydride ( $32.8 \mathrm{mmol}, 2$ equiv.) in anhydrous THF (100 mL, $4 \mathrm{vol})$ at $0-5{ }^{\circ} \mathrm{C}$ for a period of $1 \mathrm{~h}$. The reaction mixture was thereafter allowed to stir at ambient temperature for 2-3 h. Next, the reaction mixture was cooled to $0-5{ }^{\circ} \mathrm{C}$ and quenched slowly with ethyl acetate $(250 \mathrm{~mL}, 10$ vol) followed by water $(100 \mathrm{~mL}, 4 \mathrm{vol})$. The reaction mixture was filtered through celite and ethyl acetate $(50 \mathrm{~mL}, 2$ vol) was used to wash the celite bed. The organic layer was washed with water $(100 \mathrm{~mL}, 4 \mathrm{vol})$, dried over anhydrous Na2SO4, and filtered. Evaporation of the solvent under reduced pressure resulted in $99 \%$ yield of the crude alcohol as a pale yellow oil, which was used in the next step.

4-(4-(Bis(2-chloroethyl)amino)phenyl)butyl carbonochloridate (4)

DMF (1.4 g) and sodium carbonate ( $75.79 \mathrm{mmol}, 1.1$ equiv.) were added to a solution of triphosgene (37.89 mmol, 0.55 equiv.) in toluene (300 mL, 15 vol) at ambient temperature. The reaction mixture was cooled to $0-5{ }^{\circ} \mathrm{C}$ and maintained at the same temperature for $30 \mathrm{~min}$. Next, a solution of $3(68.9 \mathrm{mmol}, 1.0$ equiv.) in toluene (100 $\mathrm{mL}, 5$ vol) was added to the stirred reaction mixture at $0-5{ }^{\circ} \mathrm{C}$ during $30 \mathrm{~min}$. This reaction mixture was stirred for $4-5 \mathrm{~h}$ at room temperature. The reaction mixture was filtered thereafter and the solid was washed with toluene $(100 \mathrm{~mL}, 5 \mathrm{vol})$. Evaporation of the solvent under reduced pressure resulted in the chloroformate 4 with a $64 \%$ yield as a yellow viscous liquid, which was used for the synthesis of carbamates (compounds $5 a-c)$.

General procedure for the preparation of compounds ( $2 a-c$ and $5 a-c)$

To a solution of aminoalkylbenzene sulfonamide (1.0 equiv.) in acetonitrile ( $225 \mathrm{~mL}, 15$ vol) and $\mathrm{N}, \mathrm{N}$-diisopropylethylamine (2.5 equiv.) a solution of compound $1(2 \mathrm{a}-\mathrm{c}$ ) or compound $4(5 a-c)$ (1.0 equiv.) in acetonitrile $(75 \mathrm{~mL}, 5$ vol) was added over a period of $1 \mathrm{~h}$ and stirred overnight at ambient temperature. After completion, the reaction mixture was concentrated and the residue obtained was dissolved in ethyl acetate (150 $\mathrm{mL}, 10 \mathrm{vol})$. The organic layer was successively washed with $2 \mathrm{~N} \mathrm{HCl}$ solution $(100 \mathrm{~mL} \times$ 2) in water, dried over anhydrous $\mathrm{Na}_{2} \mathrm{SO}_{4}$, and filtered. After evaporation of the solvent under reduced pressure a pale yellow solid was obtained as a crude product. This crude product was purified with column chromatography using a silica gel (40\% ethyl acetate in hexane) to obtain compound $2 a-c$ and $5 a-c$ in a $51-87 \%$ yield. 
General procedure for amination of 3-Chlorobenzotriazine-1,4-di-N-oxides (7 and 10) 4-(2-Aminoethyl) benzene sulfonamide ( $8.25 \mathrm{mmol}, 3.0$ equiv.) was added to a stirring solution of 3 chlorobenzotriazine-1,4-di-N-oxide $(2.75 \mathrm{mmol}, 1.0$ equiv.) in dimethoxyethane $(30 \mathrm{~mL})$ and the mixture was stirred overnight at reflux temperature. The next day mixture was cooled to room temperature and concentrated under vacuum, after which the residue was dissolved in ammonium hydroxide solution and extracted with ethyl acetate. The organic layer was dried over $\mathrm{Na}_{2} \mathrm{SO}_{4}$, filtered and concentrated under vacuum. The residue was purified by chromatography using a silica gel with methylene chloride-methanol $98: 2 \mathrm{v}$ - $\mathrm{v}$ as an eluent to obtain the expected compound as a yellow powder with an $85-94 \%$ yield.

General procedure for oxidation (8 and 11)

Hydrogen peroxide (12.9 mmol, 10 equiv.) was added dropwise to a stirred solution of trifluoroacetic anhydride (12.9 mmol, 10 equiv.) in $\mathrm{DCM}$ at $0{ }^{\circ} \mathrm{C}$. This reaction mixture was stirred at $0{ }^{\circ} \mathrm{C}$ for $5 \mathrm{~min}$, warmed to room temperature for $10 \mathrm{~min}$, and cooled to 5 ${ }^{\circ} \mathrm{C}$. Next, the mixture was added to a stirred solution of mono oxide (1.29 mmol, 1.0 equiv.) in $\mathrm{DCM}$ at $0{ }^{\circ} \mathrm{C}$ and stirred at room temperature for 2-3 days. The reaction mixture was carefully diluted with water and basified with aqueous $\mathrm{NH}_{4} \mathrm{OH}$ and extracted with $\mathrm{CHCl}_{3}$. The organic fraction was dried over anhydrous $\mathrm{Na}_{2} \mathrm{SO}_{4}$, filtered and evaporated to obtain the residue. This residue was purified by chromatography using a silica gel with methylene chloride-methanol $98: 2 \mathrm{v}$ - $\mathrm{v}$ as an eluent to obtain the expected compound as an orange red powder with a 46-96\% yield.

General procedure for synthesis of ATRi derivatives (12 and 13)

A solution of VE-821 or VE-822 (0.54 mmol, 1.0 equiv.) and triethylamine (1.62 mmol, 3.0 equiv.) in $10 \mathrm{~mL}$ of methylene chloride was added to a mixture of chlorosulfonyl isocyanate ( $0.68 \mathrm{mmol}, 1.2$ equiv.) and tert-butanol ( $0.648 \mathrm{mmol}, 1.2$ equiv.) in $2 \mathrm{~mL}$ of methylene chloride. The mixture was stirred at room temperature for $1.0 \mathrm{~h}$, diluted with ethyl acetate, and washed with water. The organic layer was then dried over anhydrous $\mathrm{Na}_{2} \mathrm{SO}_{4}$, filtered and concentrated under vacuum. The residue was purified by chromatography with a silica gel and methylene chloride-methanol 98:2 as an eluent. This intermediate was thereafter diluted in a solution of trifluoro acetic acid in methylene chloride (20\% vol.) and stirred at room temperature for $6 \mathrm{~h}$. Next, the mixture was concentrated under vacuum and co-evaporated with diethyl ether multiple times to obtain the expected compound with a 58-65\% yield.

General procedure for synthesis of temozolomide derivatives

To a slurry of 3-methyl-4-oxo-3,4-dihydroimidazo[5,1-d][1,2,3,5] tetrazine-8-carboxylic acid (1.0 mmol, 1.0 equiv.) in DCM, BOP (1.0 mmol, 1.0 equiv.), amine (1.1 mmol, 1.1 equiv.) and triethylamine ( $2.5 \mathrm{mmol}, 2.5$ equiv.) were added. This reaction mixture was 
stirred overnight at room temperature and filtered to obtain the expected compounds with a $41-95 \%$ yield.

4-(4-(Bis(2-chloroethyl)amino)phenyl)-N-(4-sulfamoylphenyl)butanamide (2a) mp: $155-157^{\circ} \mathrm{C} ;{ }^{1} \mathrm{H}$ NMR (400 MHz, DMSO-d 6 ), $\delta 10.22$ (s, $\left.1 \mathrm{H}\right), 7.73(\mathrm{~d}, J=4.4,4 \mathrm{H}), 7.23$ $(\mathrm{s}, 2 \mathrm{H}), 7.05(\mathrm{~d}, J=8.7,2 \mathrm{H}), 6.67(\mathrm{~d}, J=8.7,2 \mathrm{H}), 3.70(\mathrm{~d}, J=8.6,8 \mathrm{H}), 2.54-2.50(\mathrm{~m}, 2 \mathrm{H})$, $2.34(\mathrm{t}, J=8.6,2 \mathrm{H}), 1.90-1.78(\mathrm{~m}, 2 \mathrm{H}) ;{ }^{13} \mathrm{C}$ NMR (101 MHz, DMSO-d 6 ), $\delta 171.68,144.46$, $142.23,138.03,129.53,126.65,118.51,111.90,52.22$, 41.17, 35.84, 33.54, 26.87; MS $\left(\mathrm{ESI}^{+}\right) \mathrm{m} / \mathrm{z} \quad 458.11[\mathrm{M}+\mathrm{H}]^{+}, 460.10[\mathrm{M}+2]^{+}$. HRMS $\left(E S I^{+}\right)[\mathrm{M}+\mathrm{H}]^{+}$calculated for $\left[\mathrm{C}_{20} \mathrm{H}_{26} \mathrm{~N}_{3} \mathrm{O}_{3} \mathrm{SCl}_{2}\right]+:$ 458.1072, found: 458.1075 .

4 -(4-(bis(2-chloroethyl)amino)phenyl)-N-(4-sulfamoylbenzyl)butanamide (2b) mp: $130-132{ }^{\circ} \mathrm{C} ;{ }^{1} \mathrm{H}$ NMR $\left(400 \mathrm{MHz}, \mathrm{DMSO}_{-} \mathrm{d}_{6}\right), \delta 8.41(\mathrm{t}, J=5.9,1 \mathrm{H}), 7.76(\mathrm{~d}, J=8.3$, $2 \mathrm{H}), 7.41(\mathrm{~d}, J=8.3,2 \mathrm{H}), 7.31(\mathrm{~s}, 2 \mathrm{H}), 7.02(\mathrm{~d}, J=8.6,2 \mathrm{H}), 6.66(\mathrm{~d}, J=8.6,2 \mathrm{H}), 4.31(\mathrm{~d}, J$ $=5.9,2 \mathrm{H}), 3.69(\mathrm{~s}, 8 \mathrm{H}), 2.45(\mathrm{t}, J=7.5,2 \mathrm{H}), 2.15(\mathrm{t}, J=7.5,2 \mathrm{H}), 1.82-0.72(\mathrm{~m}, 2 \mathrm{H}) ;{ }^{13} \mathrm{C}$ NMR $(101 \mathrm{MHz}$, DMSO-d 6$), \delta 172.15,144.42,143.92,142.54,129.86,129.33,127.46$, 125.68, 111.89, 52.22, 41.67, 41.17, 34.84, 33.66, 27.39; MS (ESI+) m/z 472.12 [M+H]+, $474.12[\mathrm{M}+2]^{+}$. HRMS $\left(E S I^{+}\right)[\mathrm{M}+\mathrm{H}]^{+}$calculated for $\left[\mathrm{C}_{21} \mathrm{H}_{28} \mathrm{~N}_{3} \mathrm{O}_{3} \mathrm{SCl}_{2}\right]^{+}: 472.1228$, found: 472.1236 .

4-(4-(Bis(2-chloroethyl)amino)phenyl)-N-(4-sulfamoylphenethyl)butanamide (2c) $\mathrm{mp}: 108-110{ }^{\circ} \mathrm{C} ;{ }^{1} \mathrm{H}$ NMR $\left(400 \mathrm{MHz}, \mathrm{DMSO}-\mathrm{d}_{6}\right), \delta 7.89(\mathrm{t}, J=5.6,1 \mathrm{H}), 7.74(\mathrm{~d}, J=8.3$, $2 \mathrm{H}), 7.38(\mathrm{~d}, J=8.3,2 \mathrm{H}), 7.30(\mathrm{~s}, 2 \mathrm{H}), 7.00(\mathrm{~d}, J=8.6,2 \mathrm{H}), 6.66(\mathrm{~d}, J=8.6,2 \mathrm{H}), 3.70(\mathrm{~d}, J$ $=8.9,8 \mathrm{H}), 3.29(\mathrm{dd}, J=13.0,5.6,2 \mathrm{H}), 2.78(\mathrm{t}, J=7.5,2 \mathrm{H}), 2.41(\mathrm{t}, J=7.5,2 \mathrm{H}), 2.08-1.98$ $(\mathrm{m}, 2 \mathrm{H}), 1.76-1.65(\mathrm{~m}, 2 \mathrm{H}) ;{ }^{13} \mathrm{C}$ NMR $\left(101 \mathrm{MHz}, \mathrm{DMSO}-\mathrm{d}_{6}\right), \delta 171.95,144.39,143.80$, $142.01,129.89,129.21,125.67,111.87,52.23,41.17,34.87,33.61,27.32$; MS (ESI+) $\mathrm{m} / \mathrm{z} 486.14[\mathrm{M}+\mathrm{H}]^{+}, 488.14[\mathrm{M}+2]^{+}$. HRMS $\left(E S I^{+}\right)[\mathrm{M}+\mathrm{H}]^{+}$calculated for $\left[\mathrm{C}_{22} \mathrm{H}_{30} \mathrm{~N}_{3} \mathrm{O}_{3} \mathrm{SCl}_{2}\right]^{+}$: 486.1385, found 486.1387 .

4-(4-(Bis(2-chloroethyl)amino)phenyl)butyl (4-sulfamoylphenyl)carbamate(5a)

$\mathrm{mp}:{ }^{156-158}{ }^{\circ} \mathrm{C} ;{ }^{1} \mathrm{H}$ NMR (400 MHz, DMSO-d $), \delta 10.01(\mathrm{~s}, 1 \mathrm{H}), 7.76-7.69(\mathrm{~m}, 2 \mathrm{H})$, $7.64-7.56(\mathrm{~m}, 2 \mathrm{H}), 7.22(\mathrm{~s}, 2 \mathrm{H}), 7.04(\mathrm{~d}, J=8.7,2 \mathrm{H}), 6.66(\mathrm{~d}, J=8.7,2 \mathrm{H}), 4.12(\mathrm{t}, J=6.0$, $2 \mathrm{H}), 3.77-3.63(\mathrm{~m}, 8 \mathrm{H}), 1.70-1.54(\mathrm{~m}, 4 \mathrm{H}) ;{ }^{13} \mathrm{C}$ NMR (101 MHz, DMSO-d $\left.\mathrm{d}_{6}\right), \delta 153.50$, 144.40, 142.34, 137.48, 130.06, 129.30, 126.77, 117.50, 111.89, 64.43, 52.22, 41.17, 33.68, 28.09, 27.62; MS $\left(\mathrm{ESI}^{+}\right) \mathrm{m} / \mathrm{z} 488.12[\mathrm{M}+\mathrm{H}]^{+}, 490.12[\mathrm{M}+2]^{+}$. HRMS $\left.(\mathrm{ESI})^{+}\right)[\mathrm{M}+\mathrm{H}]^{+}$ calculated for $\left[\mathrm{C}_{21} \mathrm{H}_{28} \mathrm{~N}_{3} \mathrm{O}_{4} \mathrm{SCl}_{2}\right]+: 488.1178$, found: 488.1184 .

4-(4-(Bis(2-chloroethyl)amino)phenyl)butyl (4-sulfamoylbenzyl)carbamate(5b) mp: 98-100 ${ }^{\circ} \mathrm{C} ;{ }^{1} \mathrm{H}$ NMR (400 MHz, DMSO-d 6 ), $\delta 7.76(t, J=8.5,3 \mathrm{H}), 7.41(\mathrm{~d}, J=8.5,2 \mathrm{H})$, $7.31(\mathrm{~s}, 2 \mathrm{H}), 7.02(\mathrm{~d}, J=8.6,2 \mathrm{H}), 6.66(\mathrm{~d}, J=8.6,2 \mathrm{H}), 4.23(\mathrm{~d}, J=6.1,2 \mathrm{H}), 3.98(\mathrm{~s}, 2 \mathrm{H})$, 3.75-3.63 (m, 8H), $2.47(\mathrm{~s}, 2 \mathrm{H}), 1.55(\mathrm{~m}, 4 \mathrm{H}) ;{ }^{13} \mathrm{C}$ NMR (101 MHz, DMSO-d $\left.\mathrm{d}_{6}\right), \delta 156.63$, 
$144.37,143.97,142.61,130.13,129.26,127.27,125.70,111.88,63.84,52.23,43.37$, 41.18, 33.68, 28.31, 27.64; MS $\left(E S I^{+}\right) \mathrm{m} / \mathrm{z} 502.13[\mathrm{M}+\mathrm{H}]^{+}, 504.13[\mathrm{M}+2]^{+}$. HRMS $\left(E S I^{+}\right)$ $[\mathrm{M}+\mathrm{H}]^{+}$calculated for $\left[\mathrm{C}_{22} \mathrm{H}_{30} \mathrm{~N}_{3} \mathrm{O}_{4} \mathrm{SCl}_{2}\right]^{+}: 502.1334$, found: 502.1338 .

4-(4-(Bis(2-chloroethyl)amino)phenyl)butyl (4-sulfamoylphenethyl)carbamate(5c) mp: $100-102{ }^{\circ} \mathrm{C} ;{ }^{1} \mathrm{H}$ NMR $\left(400 \mathrm{MHz}, \mathrm{DMSO}_{-} \mathrm{d}_{6}\right), \delta 7.77-7.70(\mathrm{~m}, 2 \mathrm{H}), 7.37(\mathrm{~d}, \mathrm{~J}=8.2$, $2 \mathrm{H}), 7.30(\mathrm{~s}, 2 \mathrm{H}), 7.19(\mathrm{t}, J=5.5,1 \mathrm{H}), 7.02(\mathrm{~d}, J=8.6,2 \mathrm{H}), 6.66(\mathrm{~d}, J=8.6,2 \mathrm{H}), 3.94(\mathrm{~d}, J$ $=5.5,2 \mathrm{H}), 3.69(\mathrm{~s}, 8 \mathrm{H}), 3.21(\mathrm{dd}, J=13.3,6.6,2 \mathrm{H}), 2.76(\mathrm{dd}, J=16.9,9.8,2 \mathrm{H}), 2.46(\mathrm{~s}$, $2 \mathrm{H}), 1.52(\mathrm{~s}, 4 \mathrm{H}) ;{ }^{13} \mathrm{C}$ NMR $\left(101 \mathrm{MHz}, \mathrm{DMSO}-\mathrm{d}_{6}\right), \delta 156.30,144.37,143.58,142.03$, $130.15,129.19,125.67,111.88,63.51,52.23,41.28,35.09,33.67,28.32,27.63$; MS $\left(E S I^{+}\right) \quad m / z \quad 516.15[\mathrm{M}+\mathrm{H}]^{+}, 518.15[\mathrm{M}+2]^{+}$. HRMS $\left(E S I^{+}\right)[\mathrm{M}+\mathrm{H}]^{+}$calculated for $\left[\mathrm{C}_{23} \mathrm{H}_{32} \mathrm{~N}_{3} \mathrm{O}_{4} \mathrm{SCl}_{2}\right]^{+}:$516.1491, found: 516.1490 .

3-(4-Sulfamoylphenethylamino) benzo [e][1,2,4] triazine 1-oxide (7)

Compound 7 was synthesized from 6 by a general amination method and resulted in a yellow solid with a yield of $94 \%$. mp: $250-252{ }^{\circ} \mathrm{C} ;{ }^{1} \mathrm{H}$ NMR (400 MHz, DMSO-d $\left.\mathrm{d}_{6}\right), \delta 8.15$ $(\mathrm{s}, 1 \mathrm{H}), 8.13(\mathrm{~s}, 1 \mathrm{H}), 8.03(\mathrm{~s}, 1 \mathrm{H}), 7.82-7.71(\mathrm{~m}, 3 \mathrm{H}), 7.60(\mathrm{~d}, J=8.0,1 \mathrm{H}), 7.47(\mathrm{~d}, J=8.0$, $2 \mathrm{H}), 7.38-7.30(\mathrm{~m}, 1 \mathrm{H}), 7.28(\mathrm{~s}, 2 \mathrm{H}), 3.60(\mathrm{~d}, J=6.2,2 \mathrm{H}), 3.01(\mathrm{dd}, J=6.2,2 \mathrm{H}) ;{ }^{13} \mathrm{C} N M R$ $\left(101 \mathrm{MHz}, \mathrm{DMSO}-\mathrm{d}_{6}\right) \delta 158.80,143.70,142.07,135.76,129.25,125.92,124.66,119.93$, 41.92, 34.09; MS $\left(E S I^{+}\right) \mathrm{m} / \mathrm{z} 346.10[\mathrm{M}+\mathrm{H}]^{+}$. HRMS $\left(E S I^{+}\right)[\mathrm{M}+\mathrm{H}]^{+}$calculated for $\left[\mathrm{C}_{15} \mathrm{H}_{16} \mathrm{~N}_{5} \mathrm{O}_{3} \mathrm{~S}\right]^{+}:$346.0974, found: 346.0973 .

3-((4-Sulfamoylphenethyl)amino)benzo[e][1,2,4]triazine 1,4-dioxide (8)

Compound 8 was synthesized from 7 by a general oxidation method, resulting in an orange red solid with a yield of 96\%. mp: $210-212{ }^{\circ} \mathrm{C} ;{ }^{1} \mathrm{H} N M R\left(400 \mathrm{MHz}, \mathrm{DMSO}-\mathrm{d}_{6}\right), \delta$ $8.31(\mathrm{t}, J=6.1,1 \mathrm{H}), 8.22(\mathrm{~d}, J=8.1,1 \mathrm{H}), 8.13(\mathrm{~d}, J=8.1,1 \mathrm{H}), 7.97-7.89(\mathrm{~m}, 1 \mathrm{H}), 7.75(\mathrm{~d}$, $J=8.3,2 \mathrm{H}), 7.61-7.53(\mathrm{~m}, 1 \mathrm{H}), 7.47(\mathrm{~d}, J=8.3,2 \mathrm{H}), 7.29(\mathrm{~s}, 2 \mathrm{H}), 3.67(\mathrm{dd}, J=7.2,6.1$, $2 \mathrm{H}), 3.03(\mathrm{t}, J=7.2,2 \mathrm{H}) ;{ }^{13} \mathrm{C} \operatorname{NMR}\left(101 \mathrm{MHz}, \mathrm{DMSO}-\mathrm{d}_{6}\right), \delta$ 149.67, 143.19, 142.16, $138.19,135.48,130.07,129.26,127.04,125.71,121.13,116.89,41.76,34.18 ; \mathrm{MS}_{(\mathrm{ESI}}{ }^{+}$) $\mathrm{m} / \mathrm{z} 362.09[\mathrm{M}+\mathrm{H}]^{+}$. HRMS $(\mathrm{ESI}+)[\mathrm{M}+\mathrm{H}]^{+}$calculated for $\left[\mathrm{C}_{15} \mathrm{H}_{16} \mathrm{~N}_{5} \mathrm{O}_{4} \mathrm{~S}\right]^{+}: 362.0923$, found: 362.0928 .

3-(4-Sulfamoylphenethylamino)-7,8-dihydro-6H-indeno $[5,6-e][1,2,4]$ triazine 1-oxide (10)

Compound 10 was synthesized from 9 by using a general amination method, which resulted in a yellow solid with a $85 \%$ yield. $\mathrm{mp}: 238-240{ }^{\circ} \mathrm{C} ;{ }^{1} \mathrm{H}$ NMR (400 MHz, DMSO$\left.d_{6}\right), \delta 7.95(s, 1 H), 7.82(s, 1 H), 7.74(t, J=10.0,2 H), 7.49-7.39(m, 3 H), 7.28(s, 2 H)$, $3.57(\mathrm{dd}, J=13.0,6.8,2 \mathrm{H}), 3.02-2.90(\mathrm{~m}, 6 \mathrm{H}), 2.11-1.99(\mathrm{~m}, 2 \mathrm{H}) ;{ }^{13} \mathrm{C} \mathrm{NMR}(101 \mathrm{MHz}$, DMSO-d $\left.{ }^{6}\right), \delta 157.56,153.64,142.79,141.97,130.10-127.99,125.35,112.78,41.77$, 32.35, 31.60, 25.25; MS $\left(E S I^{+}\right) \mathrm{m} / \mathrm{z} 386.13[\mathrm{M}+\mathrm{H}]^{+}$. HRMS $\left(E S I^{+}\right)[\mathrm{M}+\mathrm{H}]^{+}$calculated for $\left[\mathrm{C}_{18} \mathrm{H}_{20} \mathrm{~N}_{5} \mathrm{O}_{3} \mathrm{~S}\right]^{+}:$386.1287, found: 386.1291 . 
3-((4-Sulfamoylphenethyl) amino)-7,8-dihydro-6H-indeno $[5,6-e][1,2,4]$ triazine 1,4dioxide (11)

Compound 11 was synthesized from 10 by using a general oxidation method resulting in an orange red solid with a yield of $46 \%$. mp: $218-220{ }^{\circ} \mathrm{C} ;{ }^{1} \mathrm{H} \mathrm{NMR}\left(400 \mathrm{MHz}, \mathrm{DMSO}-\mathrm{d}_{6}\right)$, $\delta 8.19(\mathrm{~s}, 1 \mathrm{H}), 7.98(\mathrm{~d}, J=24.2,2 \mathrm{H}), 7.75(\mathrm{~d}, J=7.8,2 \mathrm{H}), 7.46(\mathrm{~d}, J=7.7,2 \mathrm{H}), 7.29$ (s, $2 \mathrm{H}), 3.65(\mathrm{~d}, J=6.2,2 \mathrm{H}), 3.12-2.92(\mathrm{~m}, 6 \mathrm{H}), 2.17-1.99(\mathrm{~m}, 2 \mathrm{H}) ;{ }^{13} \mathrm{C} N M R(101 \mathrm{MHz}$, DMSO-d 6 ), $\delta$ 154.56, 149.25, 145.07, 143.22, 142.15, 129.23, 125.71, 41.76, 32.74, 31.80, 25.24; MS $\left(E S I^{+}\right) \mathrm{m} / \mathrm{z} 402.12[\mathrm{M}+\mathrm{H}]^{+}$. HRMS $\left(\mathrm{ESI}^{+}\right)[\mathrm{M}+\mathrm{H}]^{+}$calculated for $\left[\mathrm{C}_{18} \mathrm{H}_{20} \mathrm{~N}_{5} \mathrm{O}_{4} \mathrm{~S}\right]^{+}:$402.1236, found: 402.1234 .

6-(4-(Methylsulfonyl)phenyl)-N-phenyl-3-(sulfamoylamino)pyrazine-2-carboxamide (12) Compound 12 was synthesized from commercially purchased VE-821 by using the general procedure described above, which resulted in a yellow solid with an overall yield of 58\%. mp: $233-235{ }^{\circ} \mathrm{C} ;{ }^{1} \mathrm{H}$ NMR (400 MHz, DMSO-d 6 ), $\delta 11.27$ (s, 1H), 10.82 (s, $1 \mathrm{H}), 9.28(\mathrm{~s}, 1 \mathrm{H}), 8.64(\mathrm{~d}, J=8.6,2 \mathrm{H}), 8.08(\mathrm{~d}, J=8.6,2 \mathrm{H}), 7.83-7.76(\mathrm{~m}, 2 \mathrm{H}), 7.68(\mathrm{~s}$, $2 \mathrm{H}), 7.48-7.39(\mathrm{~m}, 2 \mathrm{H}), 7.23(\mathrm{dd}, J=14.0,6.6,1 \mathrm{H}), 3.30(\mathrm{~s}, 3 \mathrm{H}) ;{ }^{13} \mathrm{C}$ NMR $(101 \mathrm{MHz}$, DMSO-d 6 ), $\delta$ 164.59-163.27, 149.32-147.73, 144.46-143.38, 141.16, 139.57, 137.17136.45, 128.69, 127.46, 125.15, 122.08, 43.46; $\mathrm{MS}\left(\mathrm{ESI}^{+}\right) \mathrm{m} / \mathrm{z} 448.07[\mathrm{M}+\mathrm{H}]^{+}$. HRMS $\left(E S I^{+}\right)[\mathrm{M}+\mathrm{H}]^{+}$calculated for $\left[\mathrm{C}_{18} \mathrm{H}_{18} \mathrm{~N}_{5} \mathrm{O}_{2} \mathrm{~S}_{2}\right]^{+}: 448.0749$, found: 448.0748 .

5-(4-(Isopropylsulfonyl) phenyl)-3-(3-(4-((methylamino) methyl) phenyl) isoxazol-5-yl) pyrazin-2-carboxamide (13)

Compound 13 was synthesized from commercially purchased VE-822 by using the general procedure described above, which resulted in a yellow solid with an overall yield of 65\%. mp: $242-244{ }^{\circ} \mathrm{C} ;{ }^{1} \mathrm{H}$ NMR (400 MHz, DMSO-d 6 ), $\delta 8.94(\mathrm{~s}, 1 \mathrm{H}), 8.38(\mathrm{~d}, \mathrm{~J}=$ $8.5,2 \mathrm{H}), 8.02(\mathrm{~d}, J=8.2,2 \mathrm{H}), 7.93(\mathrm{~d}, J=8.5,2 \mathrm{H}), 7.79(\mathrm{~s}, 1 \mathrm{H}), 7.54(\mathrm{~d}, J=8.2,2 \mathrm{H}), 7.20$ $(\mathrm{s}, 2 \mathrm{H}), 6.96(\mathrm{~s}, 2 \mathrm{H}), 4.17(\mathrm{~s}, 2 \mathrm{H}), 3.54-3.38(\mathrm{~m}, 1 \mathrm{H}), 2.58(\mathrm{~s}, 3 \mathrm{H}), 1.17(\mathrm{t}, J=14.1,6 \mathrm{H})$; ${ }^{13} \mathrm{C}$ NMR $\left(101 \mathrm{MHz}, \mathrm{DMSO}-\mathrm{d}_{6}\right), \delta 167.67,162.00,151.75,142.47,141.04,139.53$, 137.62, 135.78, 129.00, 127.17, 125.69, 124.47, 102.16, 54.22, 53.49, 34.94, 15.19; MS $\left(\mathrm{ESI}^{+}\right) \mathrm{m} / \mathrm{z} 543.15[\mathrm{M}+\mathrm{H}]^{+}$. HRMS $\left(\mathrm{ESI}^{+}\right)[\mathrm{M}+\mathrm{H}]^{+}$calculated for $\left[\mathrm{C}_{24} \mathrm{H}_{27} \mathrm{~N}_{6} \mathrm{O}_{5} \mathrm{~S}_{2}\right]^{+}: 543.1484$, found: 543.1484 .

3-Methyl-4-oxo-N-(4-sulfamoylphenethyl)-3,4-dihydroimidazo[5,1-d][1,2,3,5]tetrazine8-carboxamide (15a)

Compound 15 a was synthesized from 14 by reacting it with 4-(2-aminoethyl) benzene sulfonamide using the general procedure for synthesizing temozolomide derivatives described above. This reaction resulted in a white solid with a yield of 50\%. $\mathrm{mp}$ : 195$197{ }^{\circ} \mathrm{C} ;{ }^{1} \mathrm{H}$ NMR (400 MHz, DMSO-d $), \delta 8.83(\mathrm{~s}, 1 \mathrm{H}), 8.58(\mathrm{t}, J=5.9,1 \mathrm{H}), 7.74(\mathrm{~d}, J=8.3$, $2 \mathrm{H}), 7.44(\mathrm{~d}, J=8.3,2 \mathrm{H}), 7.30(\mathrm{~s}, 2 \mathrm{H}), 3.86(\mathrm{~s}, 3 \mathrm{H}), 3.58(\mathrm{dd}, J=13.4,6.8,2 \mathrm{H}), 2.96(\mathrm{t}, J=$ 7.1, 2H); ${ }^{13} \mathrm{C}$ NMR (101 MHz, DMSO-d $), \delta 159.67,143.64,142.05,139.20,134.45$, 
130.30, 129.14, 128.46, 125.73, 36.16, 34.78; MS (ESI $\left.{ }^{+}\right) \mathrm{m} / \mathrm{z} 378.10[\mathrm{M}+\mathrm{H}]^{+}$. HRMS $\left(\mathrm{ESI}^{+}\right)$ $[\mathrm{M}+\mathrm{H}]^{+}$calculated for $\left[\mathrm{C}_{14} \mathrm{H}_{16} \mathrm{~N}_{7} \mathrm{O}_{4} \mathrm{~S}\right]^{+}: 378.0984$, found: 378.0986 .

3-Methyl-4-oxo-N-(sulfamoyloxy)-3,4-dihydroimidazo[5,1-d][1,2,3,5]tetrazine-8carboxamide (15b)

Compound $15 \mathrm{~b}$ was synthesized from 14 by reacting it with aminoxysulfonamide using the general procedure for synthesizing temozolomide derivatives described above. This reaction resulted in a white solid with a yield of $41 \%$. mp: $195-197{ }^{\circ} \mathrm{C}$; ${ }^{1} \mathrm{H}$ NMR $(400$ MHz, DMSO-d $\left.\mathrm{d}_{6}\right), \delta 8.81(\mathrm{~s}, 1 \mathrm{H}), 7.80(\mathrm{~s}, 1 \mathrm{H}), 7.67(\mathrm{~s}, 1 \mathrm{H}), 7.30(\mathrm{~s}, 2 \mathrm{H}), 3.86(\mathrm{~s}, 3 \mathrm{H}) ;{ }^{13} \mathrm{C}$ NMR $\left(101 \mathrm{MHz}, \mathrm{DMSO}-\mathrm{d}_{6}\right) \delta 161.51,139.16,134.57,130.51,128.37,45.72$.

3-Methyl-4-oxo-N-(4-sulfamoylbenzyl)-3,4-dihydroimidazo [5,1-d][1,2,3,5]tetrazine-8carboxamide (15c)

Compound $15 \mathrm{c}$ was synthesized from 14 by reacting it with 4-(aminomethyl) benzene sulfonamide hydrochloride using the general procedure for synthesizing temozolomide derivatives described above. This reaction resulted in a white solid with a yield of $88 \%$. mp: $185-187^{\circ} \mathrm{C} ;{ }^{1} \mathrm{H}$ NMR (400 MHz, DMSO-d 6 ) $\delta 9.20(\mathrm{t}, J=6.2,1 \mathrm{H}), 8.87(\mathrm{~s}, 1 \mathrm{H}), 7.77$ $(\mathrm{d}, J=8.3,2 \mathrm{H}), 7.50(\mathrm{~d}, J=8.3,2 \mathrm{H}), 7.31(\mathrm{~s}, 2 \mathrm{H}), 4.55(\mathrm{~d}, J=6.3,2 \mathrm{H}), 3.87(\mathrm{~s}, 3 \mathrm{H}) ;{ }^{13} \mathrm{C}$ NMR (101 MHz, DMSO-d $\left.)_{6}\right) \delta 159.96,143.85,142.63,142.63,138.96,134.69,130.11$, 129.28, 128.62, 127.68, 125.77, 41.94, 36.23; MS (ESI $\left.{ }^{+}\right) \mathrm{m} / \mathrm{z} 364.08[\mathrm{M}+\mathrm{H}]^{+}$. HRMS (ESI ${ }^{+}$) $[\mathrm{M}+\mathrm{H}]^{+}$calculated for $\left[\mathrm{C}_{13} \mathrm{H}_{14} \mathrm{~N}_{7} \mathrm{O}_{4} \mathrm{~S}\right]^{+}: 364.0828$, found: 364.0826 .

3-Methyl-4-oxo-N-(5-sulfamoyl-1,3,4-thiadiazol-2-yl)-3,4-dihydroimidazo[5,1d] $[1,2,3,5]$ tetrazine-8-carboxamide (15d)

Compound $15 \mathrm{~d}$ was synthesized from 14 by reacting it with 5 -amino-1, 3,4-thiadiazole2-sulfonamide hydrochloride using the general procedure for synthesizing temozolomide derivatives described above. This reaction resulted in a light yellow solid with a yield of $95 \%$. mp: $128-130{ }^{\circ} \mathrm{C} ;{ }^{1} \mathrm{H}$ NMR (400 MHz, DMSO-d 6 ), $\delta 8.63(\mathrm{~s}, 1 \mathrm{H}), 8.06$ $(\mathrm{s}, 1 \mathrm{H}), 7.81(\mathrm{~s}, 1 \mathrm{H}), 7.35(\mathrm{~s}, 2 \mathrm{H}), 3.83(\mathrm{~s}, 3 \mathrm{H}) ;{ }^{13} \mathrm{C}$ NMR (101 MHz, DMSO-d $\left.\mathrm{d}_{6}\right), \delta 171.73$, 170.89, 165.39, 161.04, 157.91, 139.53, 134.24, 127.88; MS (ESI $\left.{ }^{+}\right) \mathrm{m} / \mathrm{z} 358.01[\mathrm{M}+\mathrm{H}]^{+}$. HRMS $\left(E S I^{+}\right)[\mathrm{M}+\mathrm{H}]^{+}$calculated for $\left[\mathrm{C}_{8} \mathrm{H}_{8} \mathrm{~N}_{9} \mathrm{O}_{4} \mathrm{~S}_{2}\right]^{+}: 358.0141$, found: 358.0140 .

Synthesis of 4-(2-(3-carbamimidoylguanidino)ethyl)benzenesulfonamide hydrochloride salt (18)

4-(2-Aminoethyl)benzenesulfonamide $16(0.5 \mathrm{~g}, 1.0$ equiv.) and cyanoguanidine 17 (0.21g, 1.0 equiv.) were suspended in $\mathrm{n}$-butanol $(5.0 \mathrm{~mL})$ and treated with a $6.0 \mathrm{M}$ aqueous hydrochloric acid solution (1.0 equiv., $0.4 \mathrm{~mL}$ ). The mixture was treated at 100 ${ }^{\circ} \mathrm{C}$ overnight and the solvents were removed under vacuum. The residue was thereafter crystallized from isopropyl alcohol (IPA) to obtain compound 18 as a white solid with a $75 \%$ yield. mp: $154-159{ }^{\circ} \mathrm{C} ;{ }^{1} \mathrm{H}$ NMR (400 MHz, DMSO-d 6 ), $\delta 7.87$ (d, 2H, J = 8.4, Ar-H), 7.82 (brs, $2 \mathrm{H}$, exchangeable with $\mathrm{D} 2 \mathrm{O}), 7.50(\mathrm{~d}, 2 \mathrm{H}, J=8.4, \mathrm{Ar}-\mathrm{H}), 7.38$ (brs, $1 \mathrm{H}$, 
exchangeable with D2O), 6.62 (brs, $2 \mathrm{H}$, exchangeable with $\left.\mathrm{D}_{2} \mathrm{O}\right), 3.15(\mathrm{t}, 2 \mathrm{H}, J=6.7$ ), $2.98(t, 2 \mathrm{H}, J=6.7) ;{ }^{13} \mathrm{C} N M R\left(101 \mathrm{MHz}, \mathrm{DMSO}-\mathrm{d}_{6}\right), \delta 164.0,143.7,142.0,130.3,127.0$, 119.3, 61.4, 34.1; MS $\left(\right.$ ESI $\left.^{+}\right) \mathrm{m} / \mathrm{z} 285.11[\mathrm{M}+\mathrm{H}]^{+}$.

\section{CA inhibition assays}

To measure the CA-catalyzed $\mathrm{CO}_{2}$ hydration activity an Applied Photophysics stoppedflow instrument was used [61]. To maintain ionic strength $\mathrm{Na}_{2} \mathrm{SO}_{4}(20 \mathrm{mM})$ was used with HEPES (20 mM, pH 7.5) as a buffer and Phenol red (0.2 mM) as an indicator working at the maximum absorbance of $557 \mathrm{~nm}$, which was used to follow the initial rates of the $\mathrm{CA}$-catalyzed $\mathrm{CO}_{2}$ hydration for a duration of 10-100 s. To determine the kinetic parameters and inhibition constants varying $\mathrm{CO}_{2}$ concentrations were included (1.7-17 mM). Initial velocity was assayed with at least six traces of the initial 5-10\% of the reaction for each compound. Compounds were dissolved in distilled-deionized water $(0.01 \mathrm{nM})$. The combined enzyme solutions and compounds were incubated for $15 \mathrm{~min}$ at room temperature to allow for the E-I complex formation prior to measurements. The nonlinear least-squares method of PRISM 3 was used to estimate the inhibition constants and the mean of three independent estimations is reported. The CA isoforms included are recombinant proteins obtained in house.

\section{Biological assays}

Cells

All cell lines used were cultured in DMEM supplemented with $10 \%$ fetal bovine serum. Canine kidney epithelial MDCK cells overexpressing human CAIX (CAIX ${ }^{+}$) or a scrambled control vector (CAIX) have been described before $[37,44]$. The HCT116 constitutive CAIX knockdown cell line and its scrambled control have also been described before and were kindly provided by Professor Adrien Harris (Weatherall Institute of Molecular Medicine, University of Oxford, John Radcliffe hospital, Oxford, UK) [24, 28, 62]. Cells were exposed to hypoxic or anoxic conditions in a hypoxic chamber (MACS VA500 microaerophilic workstation, Don Whitley Scientific, UK) with 0.2 or $\leq 0.02 \% \mathrm{O}_{2}$, respectively, and $5 \% \mathrm{CO}_{2}$ and residual $\mathrm{N}_{2}$ to upregulate and activate CAIX. Normoxic cells were grown in normal incubators with $21 \% \mathrm{O}_{2}, 5 \% \mathrm{CO}_{2}$ at $37^{\circ} \mathrm{C}$.

Cell viability assays

The efficacy of the cytotoxic derivatives was compared to their respective parental compounds in cell viability assays using alamarBlue ${ }^{\circledR}$ (Invitrogen). In short, MDCK cells were seeded in 96-well plates and allowed to attach overnight. The next day plates were exposed to hypoxia and DMEM was replaced with pre-incubated hypoxic DMEM. In contrast, testing the ATRi was performed in anoxic conditions to decrease the radiosensitivity of the cells. In parallel normoxic 96-well plates were incubated in 
normal incubators with 21\% O2 and 5\% CO2. Compounds were dissolved in DMSO (0.5\%, Sigma-Aldrich) and final concentrations were made with pre-incubated hypoxic or normoxic DMEM and added to the wells after $24 \mathrm{~h}$ of exposure. To test the ATR inhibitors cells were exposed to the compounds $1 \mathrm{~h}$ prior to irradiation and the 96-well plates were irradiated (225 kV Philips X-ray tube) with 2 Gy (normoxia) or 4 Gy (anoxia). Cells were exposed to compounds for a total of $2 \mathrm{~h}$ for chlorambucil and tirapazamine, or $72 \mathrm{~h}$ for temozolomide and the ATR inhibitors, after which medium was washed off and replaced with fresh medium. For chlorambucil, tirapazamine, and ATR inhibitor derivatives cells were allowed to grow for an additional $72 \mathrm{~h}$ under normoxic conditions prior to measurement, whereas cells exposed to temozolomide derivatives remained in hypoxic conditions prior to measurement. Cells were allowed to convert alamarBlue ${ }^{\circledR}$ for $2 \mathrm{~h}$ during normoxic conditions, which corresponds with their metabolic function and is a measure for cell viability.

\section{Clonogenic assays}

Clonogenic survival of MDCK cells was determined with high cell numbers to allow for CAIX-dependent extracellular acidification [44]. These cells were exposed to temozolomide or $15 \mathrm{~b}$ for $24 \mathrm{~h}$ during normoxic or hypoxic conditions after which cells were trypsinized and reseeded in triplicate with known cell numbers. Cells were allowed to grow for 7 days to form colonies that were quantified after staining and fixation with $0.4 \%$ methylene blue in $70 \%$ ethanol. Surviving fraction was normalized to vehicle $(0.5 \%$ DMSO).

\section{Basal respiration measurements}

Oxygen Consumption Rates (OCR) were determined using the Seahorse XF96 extracellular Flux analyzer (Agilent Technologies). Cells were seeded in a XF96 cell plate with normal growth medium at an optimized cell density of $1.5 \times 10^{4}$ cells/well. Plates were placed in a $5 \% \mathrm{CO}_{2}$ incubator at $37{ }^{\circ} \mathrm{C}$ in order to let the cells attach. Subsequently cells were incubated for $18 \mathrm{~h}$ under hypoxic conditions $\left(0.2 \% \mathrm{O}_{2}\right)$. Culture medium was exchanged with DMEM containing $25 \mathrm{mM}$ d-glucose, $4 \mathrm{mM}$ l-glutamine and $1 \mathrm{mM}$ sodiumpyruvate (GIBCO, Thermo Fisher) 60 min prior to the assay and plates were placed in a $\mathrm{CO}_{2}$-free incubator at $37^{\circ} \mathrm{C}$. Prior to the first injection, baseline OCR was determined using a mixing period of $5 \mathrm{~min}$ and a measurement period of $3 \mathrm{~min}$ followed by 3 loops of mixing and measuring for 3 min each. Medium containing vehicle (PBS, Lonza), Phenformin Hydrochloride (Sigma-Aldrich), or the CAIXi conjugated phenformin derivative 18 were injected followed by several mixing and measurements cycles. Subsequently cells were washed with PBS and lysed in a 0.05\% SDS (Sigma-Aldrich) solution. Protein quantification for normalization purposes was performed using Pierce $^{\text {TM }}$ BCA Protein Assay Kit (Thermo Fisher). 
Western blot

To validate CAIX expression in the genetically modified cell lines protein immunoblotting was performed after $24 \mathrm{~h}$ of hypoxia exposure $\left(0.2 \% \mathrm{O}_{2}\right)$ as described previously [43]. Primary antibodies used included the anti-CAIX M75 antibody (kindly provided by Professor Silvia Pastorekova, Institute of Virology, Slovak Academy of Science, Slovak Republic), and anti- $\beta$-actin (MP Biomedicals, \#691001) as a reference protein.

Statistical analyses

GraphPad Prism (version 5.03) was used for all statistical analyses. For the cytotoxic compounds $I C_{50}$ values were estimated with the curve of the $\log$ (inhibitor) vs. normalized response (Variable slope). Means between groups were compared using unpaired t-tests, where $\mathrm{p}<0.05$ indicated statistical significance.

\section{Acknowledgements}

Authors acknowledge the aid of Advinus Therapeutics Ltd. for synthesis of compounds $1,2 a, 2 b, 2 c, 3,4,5 a, 5 b$ and 5c. This work was supported by METOXIA (Metastatic Tumors Facilitated by Hypoxic Micro-Environment; EU 7th Research Framework Programme - Theme HEALTH; Grant no.: 222741) NGI Pre-Seed grant ( $n^{\circ}$ 93612005), Kankeronderzoekfonds Limburg from the Health Foundation Limburg and the Dutch Cancer Society (KWF UM 2011-5020, KWF UM 2009-4454, KWF MAC 2013-6425, KWF MAC 2013-6089, KWF 2015-7635). 


\section{Supplementary data}

A

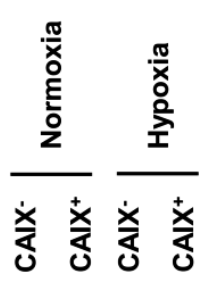

B

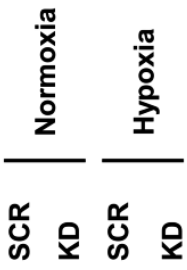

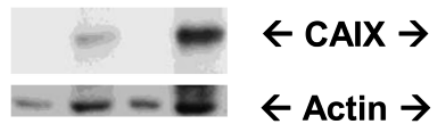

C

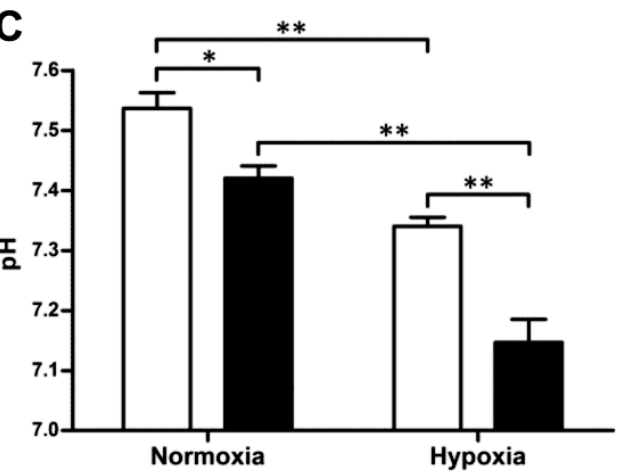

Supplementary Figure S1. CAIX protein expression during normoxia and hypoxia of the MDCK (A) and HCT116 (B) cells and $\mathrm{pH}$ measurements of MDCK cells (C). Expression of $ß$-actin was included as a reference protein. CAIX ${ }^{+}$MDCK cells are overexpressing CAIX, and CAIX cells are control cells lacking both human and canine CAIX expression. HCT116 scrambled control vector cells (SCR) show hypoxia-dependent CAIX expression, whereas CAIX knockdown (KD) cells do not. During normoxic conditions HCT116 SCR cells have no detectable levels of CAIX, since the $\operatorname{dot}(\mathrm{B})$ is an artefact on film. The $\mathrm{pH}$ of the culture medium was measured of the MDCK cells after 24 hours of normoxic or hypoxic exposure (C). Mean \pm SEM of three independent biological repeats are shown. Asterisks indicate statistical significance $\left({ }^{*} p<0.05 ;{ }^{*} p<0.01\right)$. 
Chlorambucil

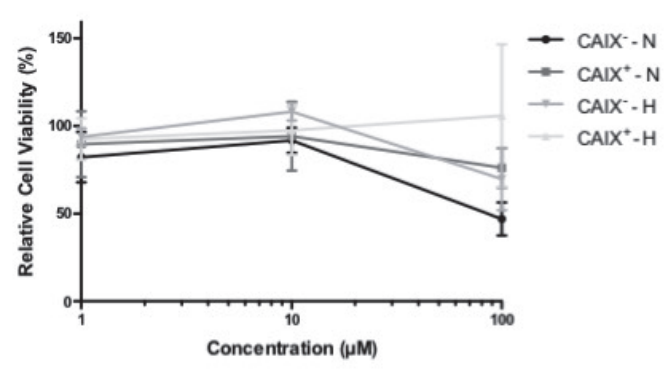

2 a

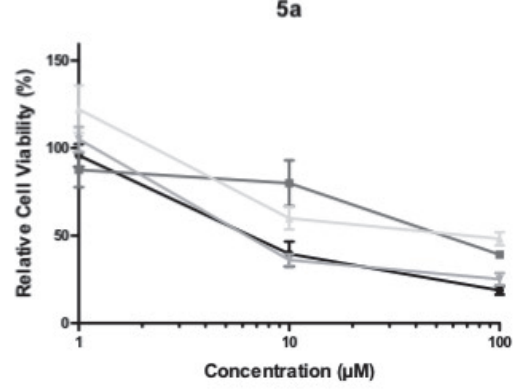

2b
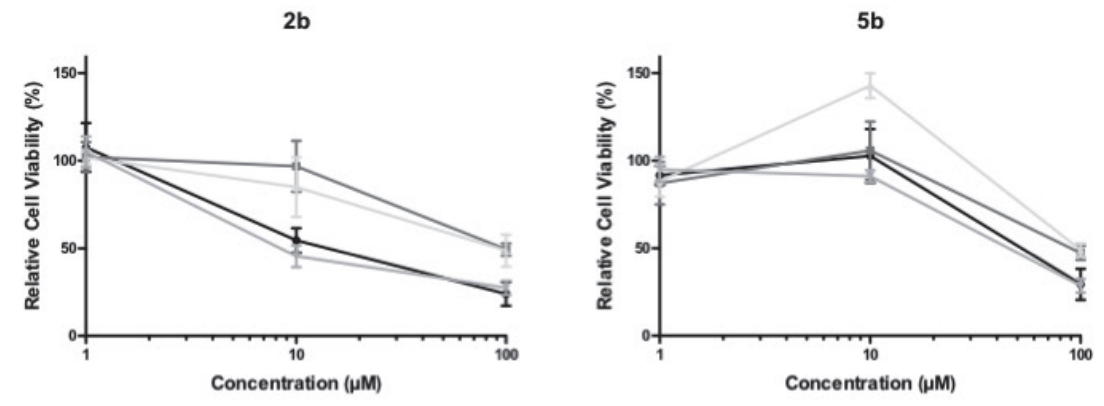

2c
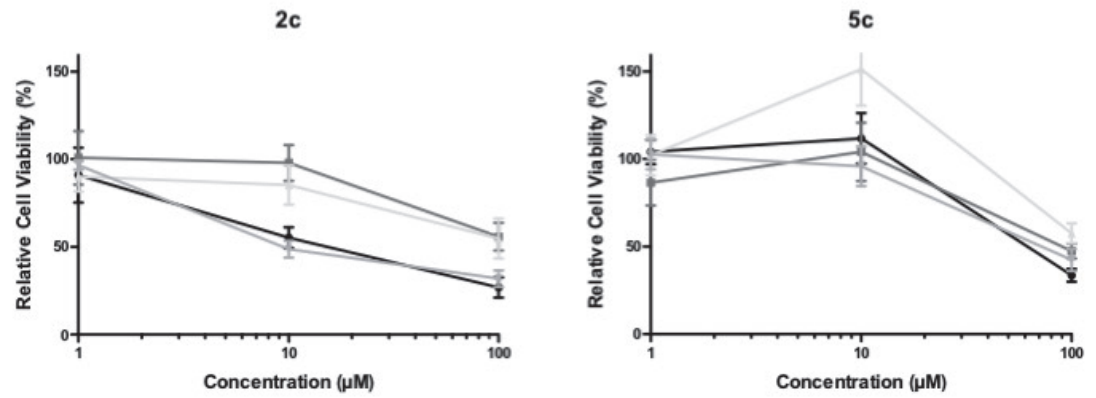

Supplementary Figure S2. Relative cell viability of $\mathrm{CAIX}^{+}$and CAIX MDCK cells exposed to increasing concentrations of chlorambucil or the CAIXi conjugated derivatives during normoxic $(\mathrm{N})$ and hypoxic $(\mathrm{H})$ conditions. Relative cell viability was normalized to vehicle control (0.5\% DMSO). Average \pm SEM of three independent biological repeats is shown. 


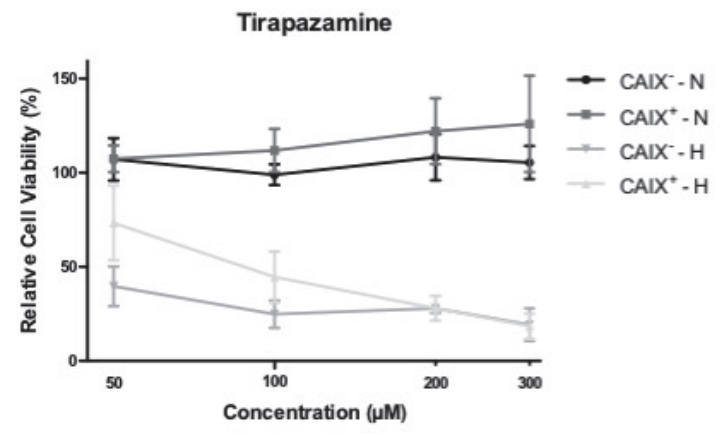

8

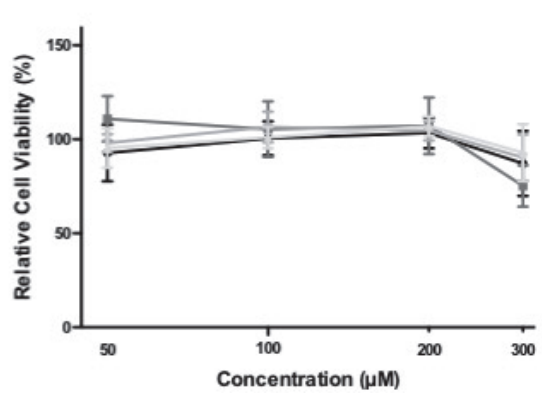

Supplementary Figure S3. Relative cell viability of CAIX ${ }^{+}$and CAIX MDCK cells exposed to increasing concentrations of tirapazamine or the CAIXi conjugated derivatives during normoxic $(\mathrm{N})$ and hypoxic $(\mathrm{H})$ conditions. Relative cell viability was normalized to vehicle control (0.5\% DMSO). Average \pm SEM of three independent biological repeats is shown. 


\section{Temozolomide}

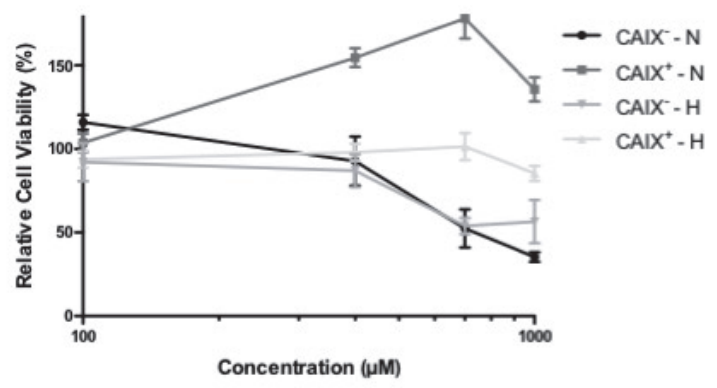

$15 a$

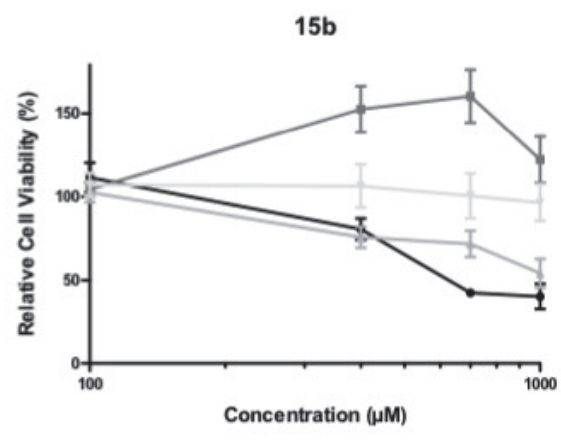

$15 c$

$15 d$
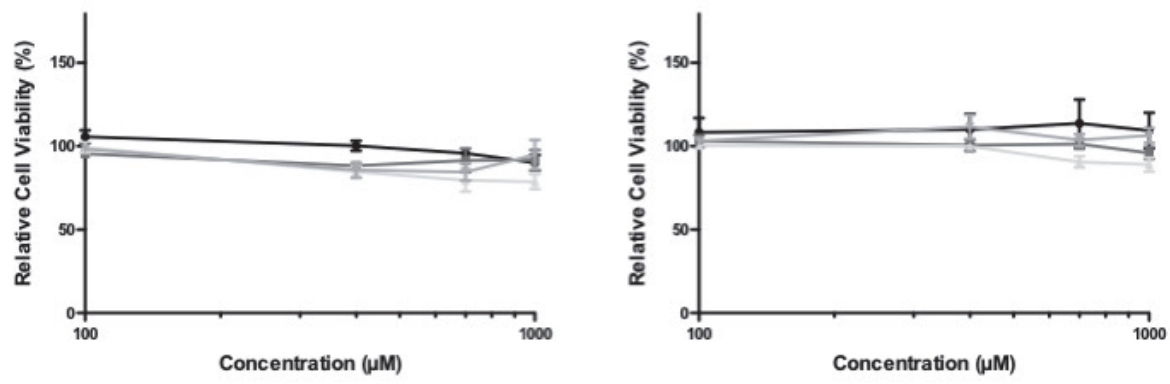

Supplementary Figure S4. Relative cell viability of CAIX ${ }^{+}$and CAIX MDCK cells exposed to increasing concentrations of temozolomide or the CAIXi conjugated derivatives during normoxic $(N)$ and hypoxic $(H)$ conditions. Relative cell viability was normalized to vehicle control (0.5\% DMSO). Average \pm SEM of three independent biological repeats is shown. 
A Normoxia
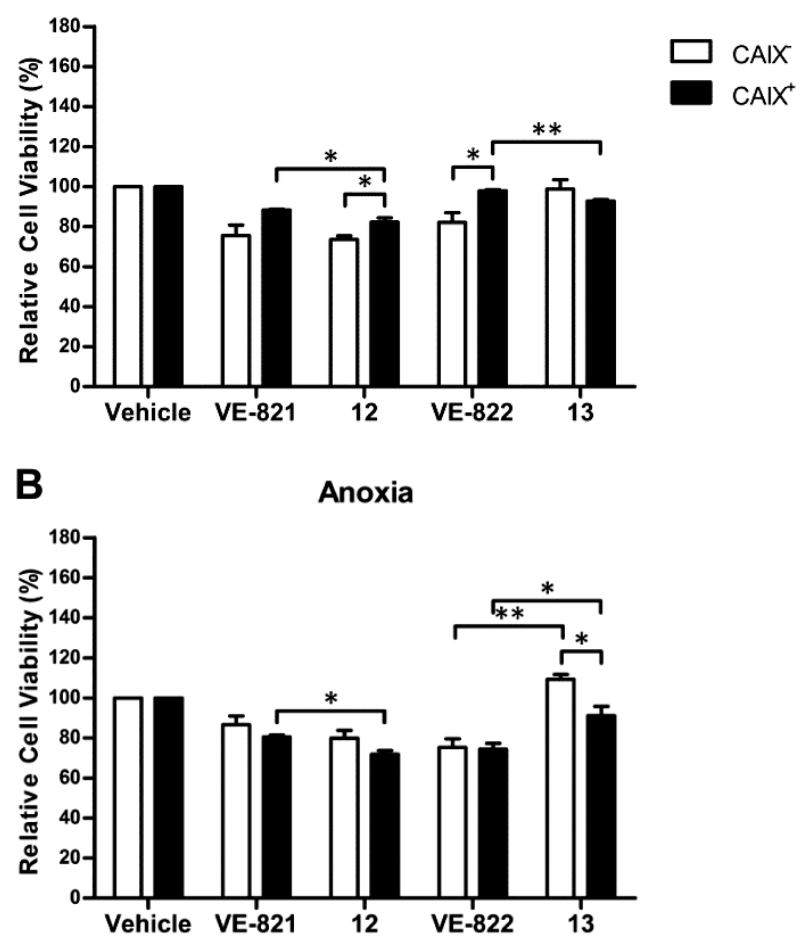

Supplementary Figure S5. Relative cell viability (\%) in MDCK CAIX and CAIX cells exposed to ATR inhibitors (VE-821 and VE-822) or the CAIXi conjugated derivatives (12 and 13) without radiation during normoxia (21\%

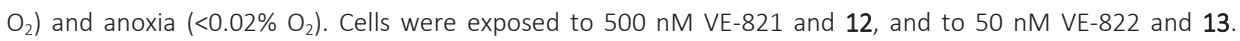
Average \pm SEM of three independent biological repeats is shown. Asterisks indicate statistical significance $\left({ }^{*} p\right.$ $<0.05 ; * * p<0.01 ; * * * p<0.001)$. 
Chapter 5
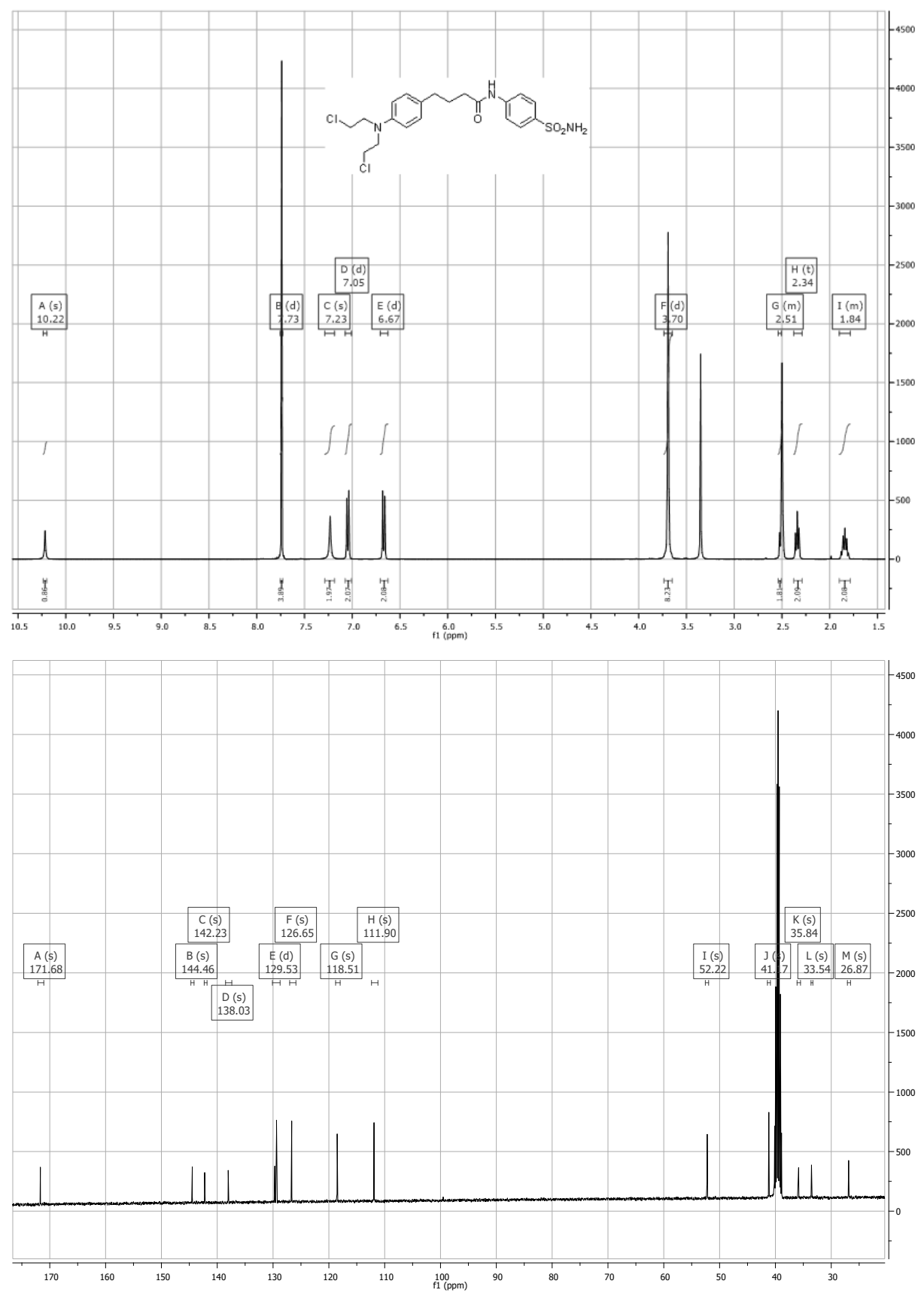

NMR spectra 1: 4-(4-(bis(2-chloroethyl)amino)phenyl)-N-(4-sulfamoylphenyl)butanamide (2a). 

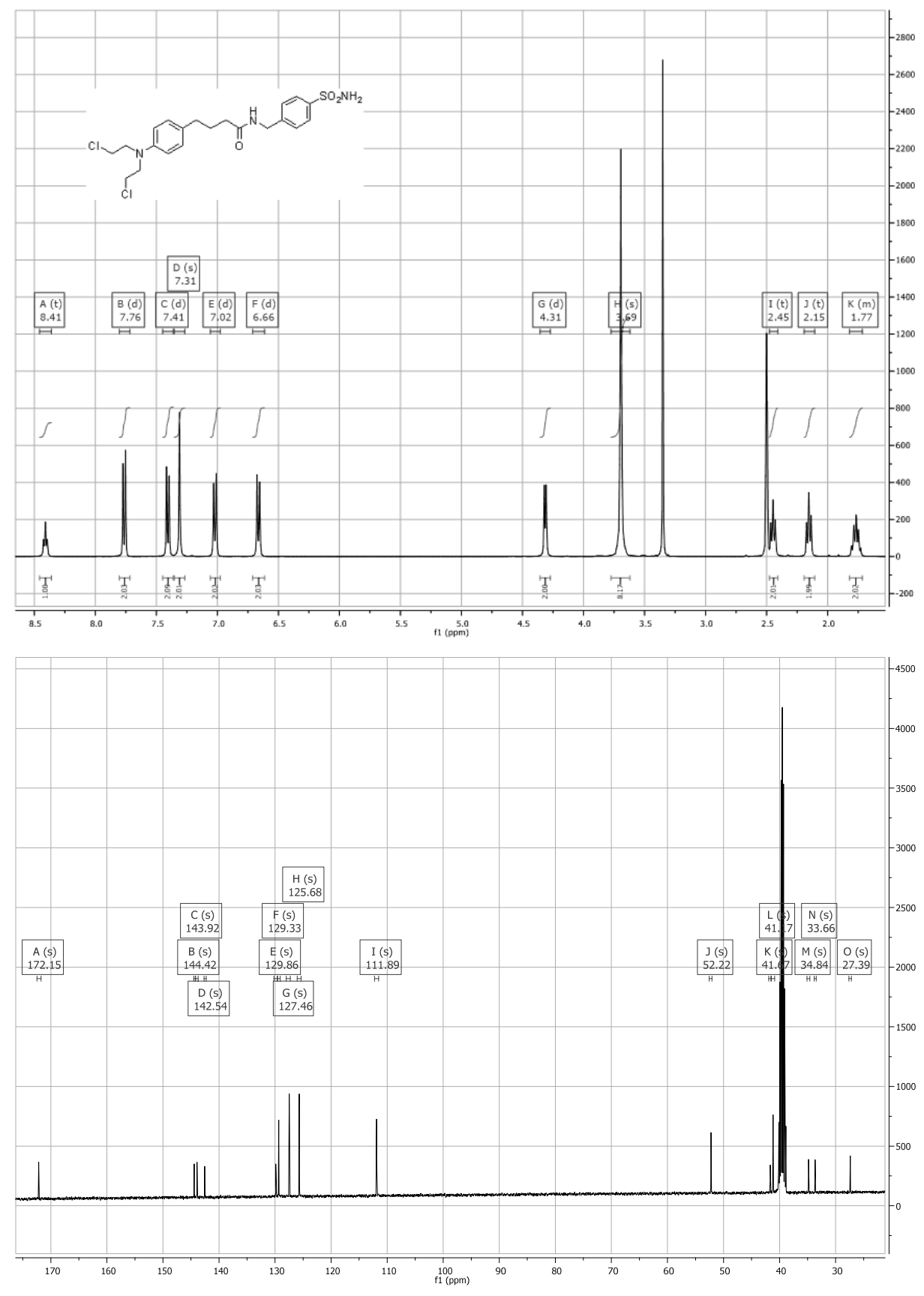

NMR spectra 2: 4-(4-(bis(2-chloroethyl)amino)phenyl)-N-(4-sulfamoylbenzyl)butanamide (2b). 
Chapter 5
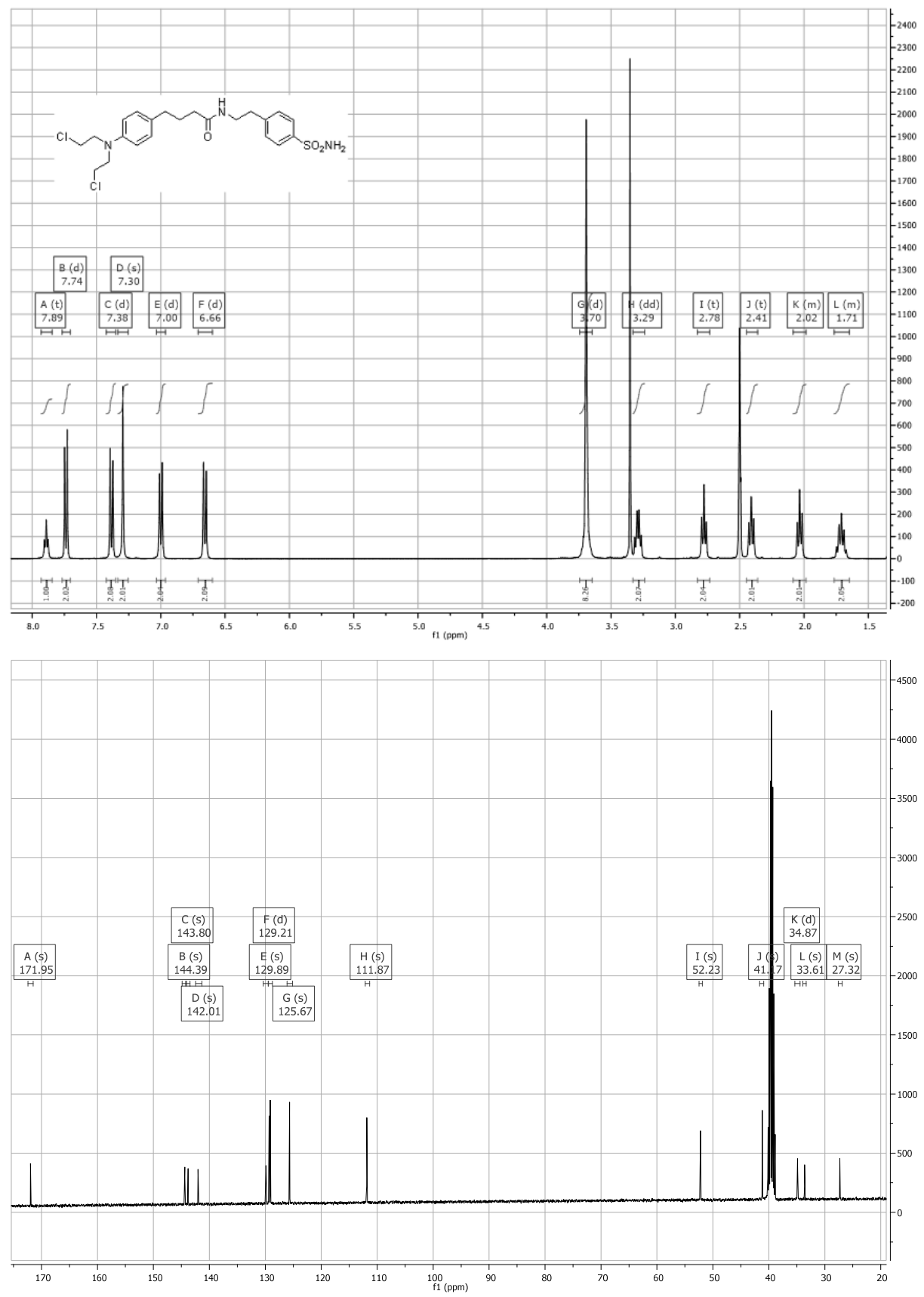

NMR spectra 3: 4-(4-(bis(2-chloroethyl)amino)phenyl)-N-(4-sulfamoylphenethyl)butanamide(2c). 

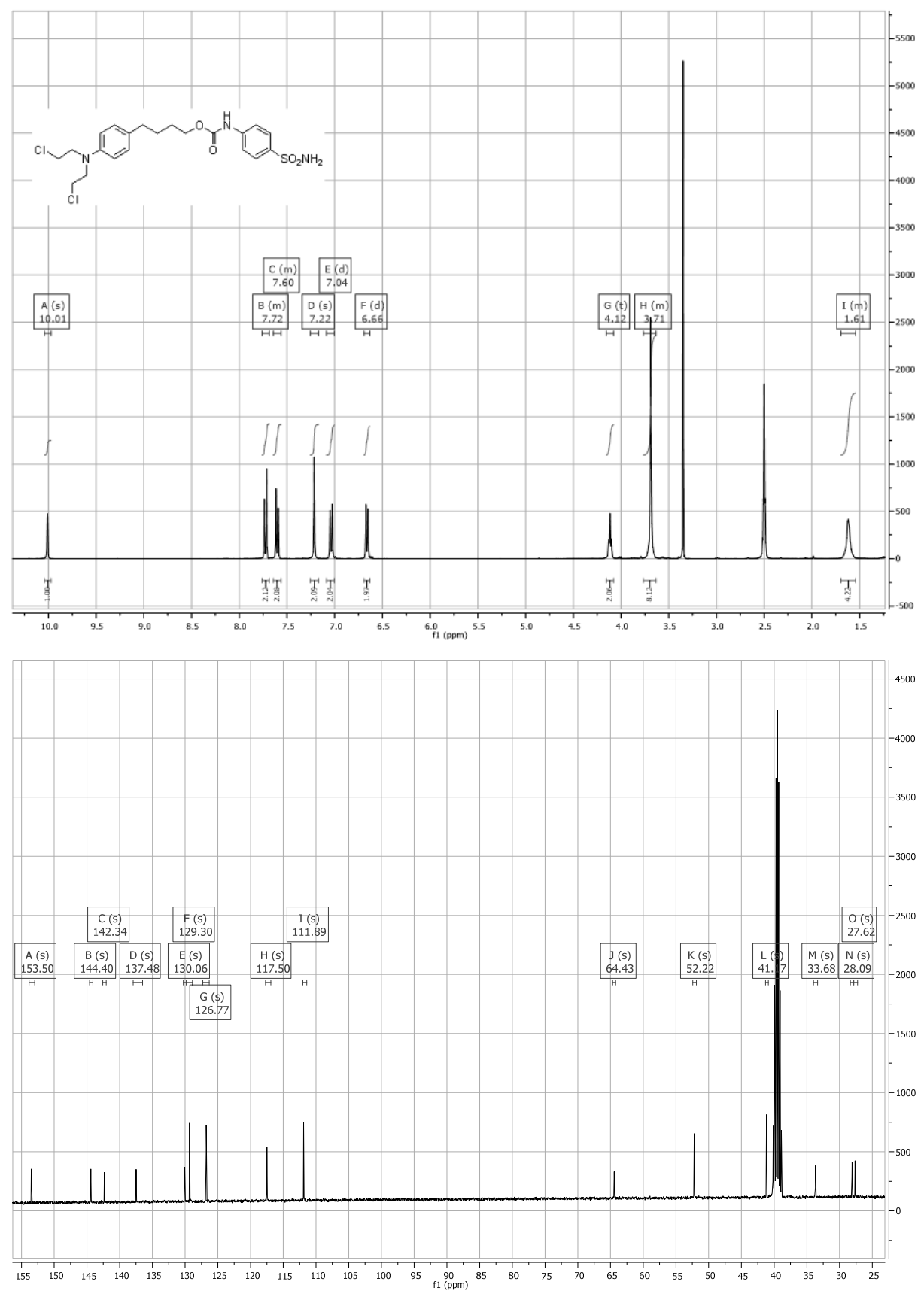

NMR spectra 4: 4-(4-(bis(2-chloroethyl)amino)phenyl)butyl (4-sulfamoylphenyl)carbamate(5a). 
Chapter 5
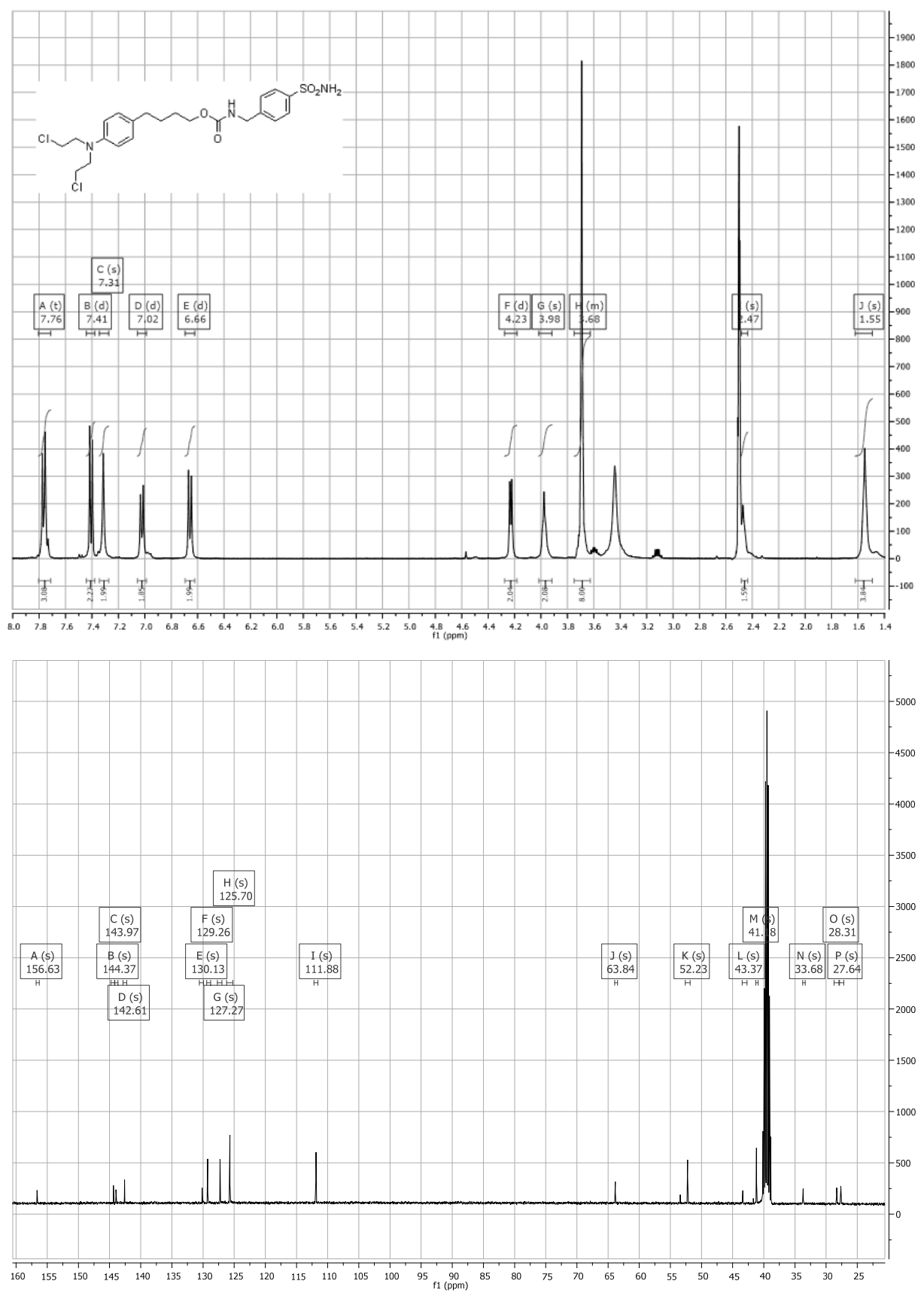

NMR spectra 5: 4-(4-(bis(2-chloroethyl)amino)phenyl)butyl (4-sulfamoylbenzyl)carbamate(5b). 

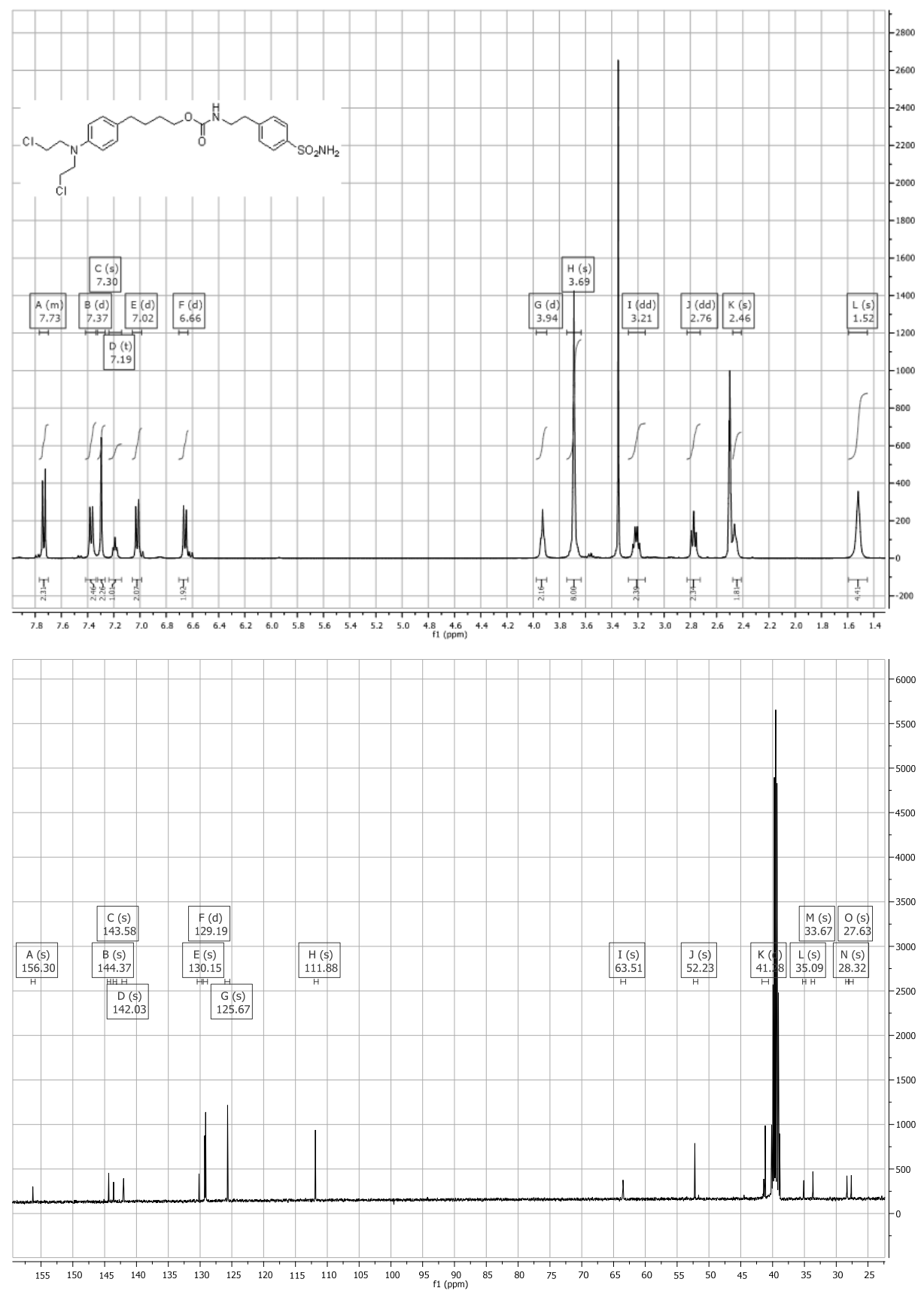

NMR spectra 6: 4-(4-(bis(2-chloroethyl)amino)phenyl)butyl (4-sulfamoylphenethyl)carbamate(5c). 

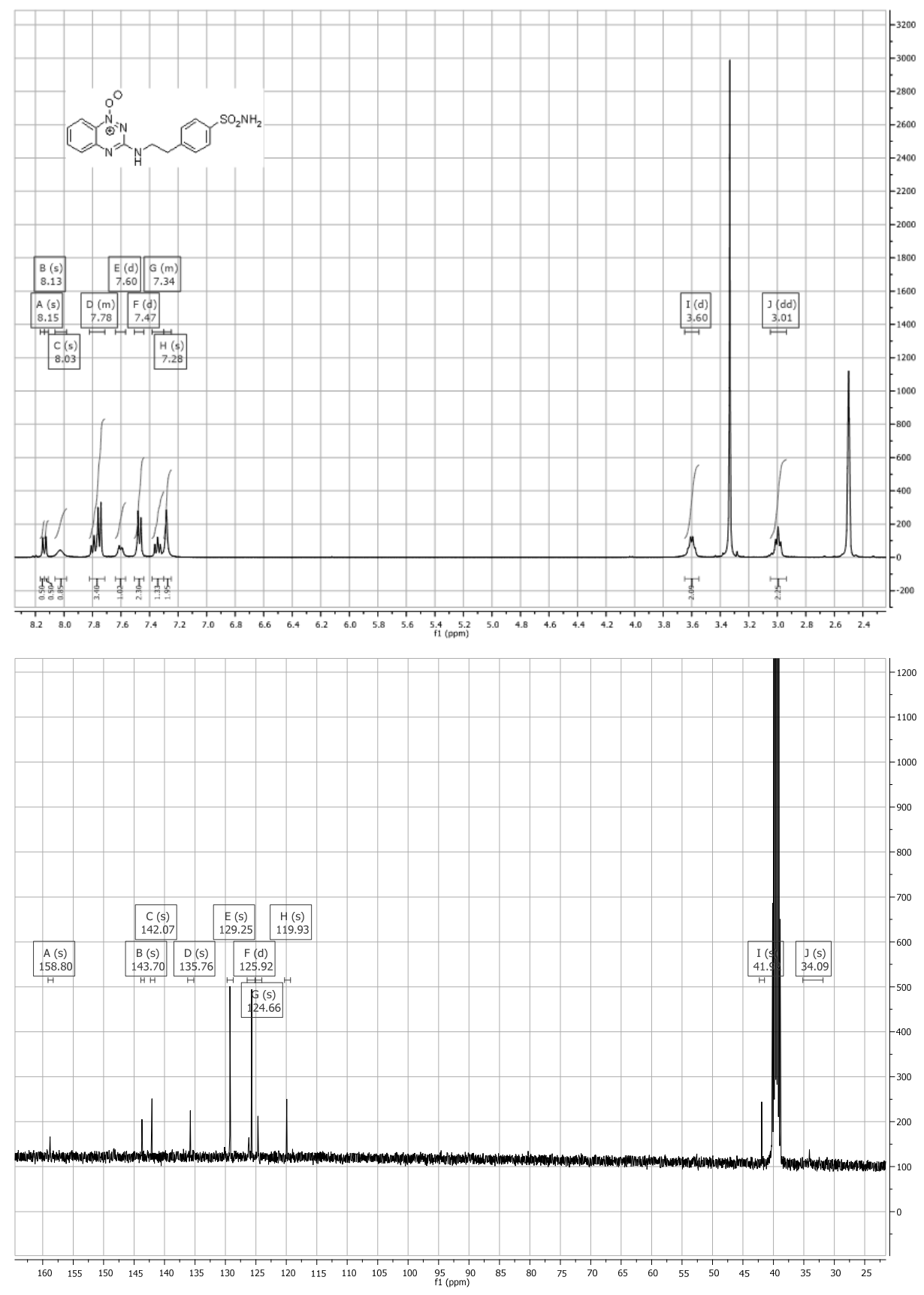

NMR spectra 7: 3-(4-sulfamoylphenethylamino) benzo [e][1,2,4] triazine 1-oxide (7). 

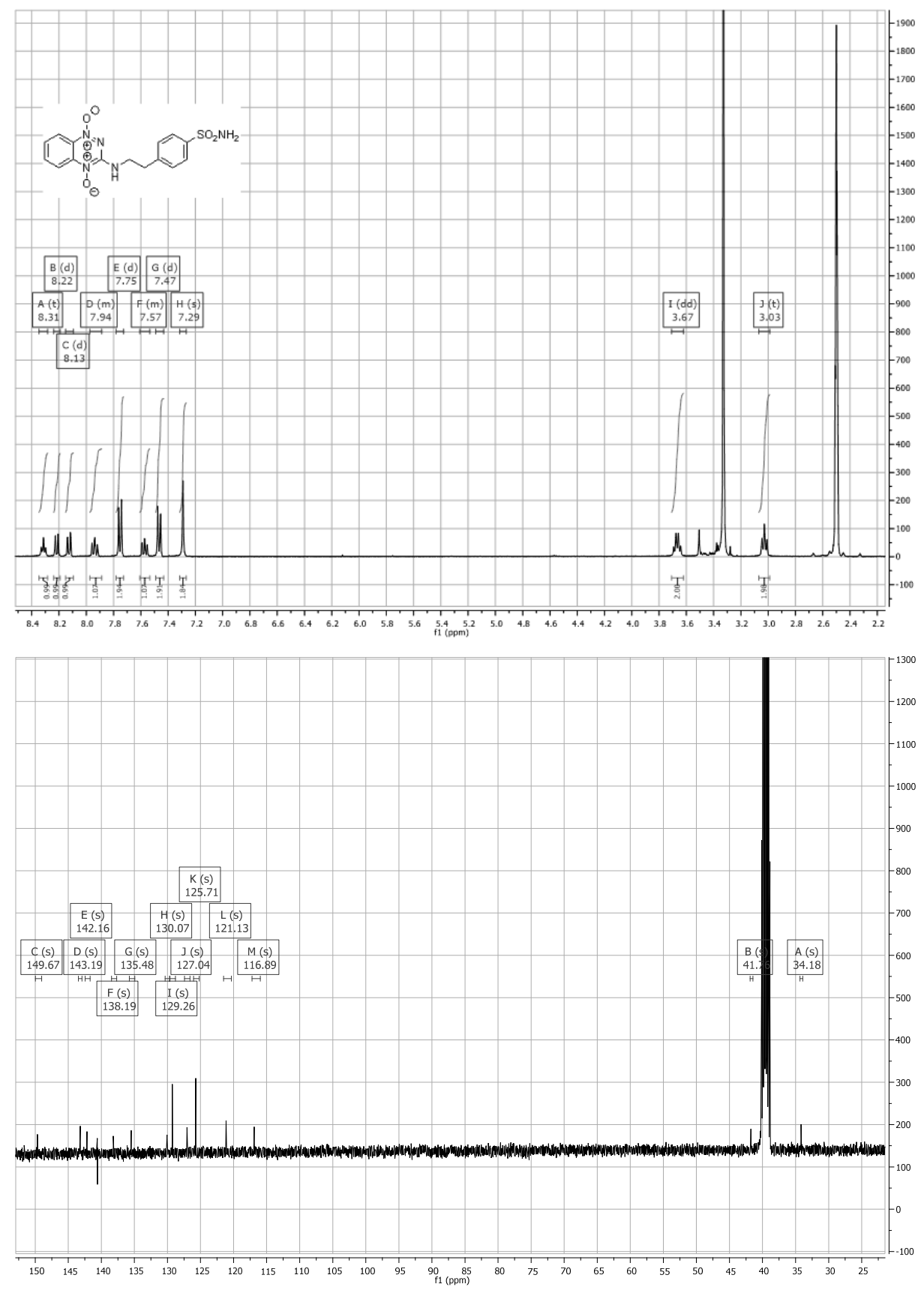

NMR spectra 8: 3-((4-sulfamoylphenethyl)amino)benzo[e][1,2,4]triazine 1,4-dioxide (8). 
Chapter 5
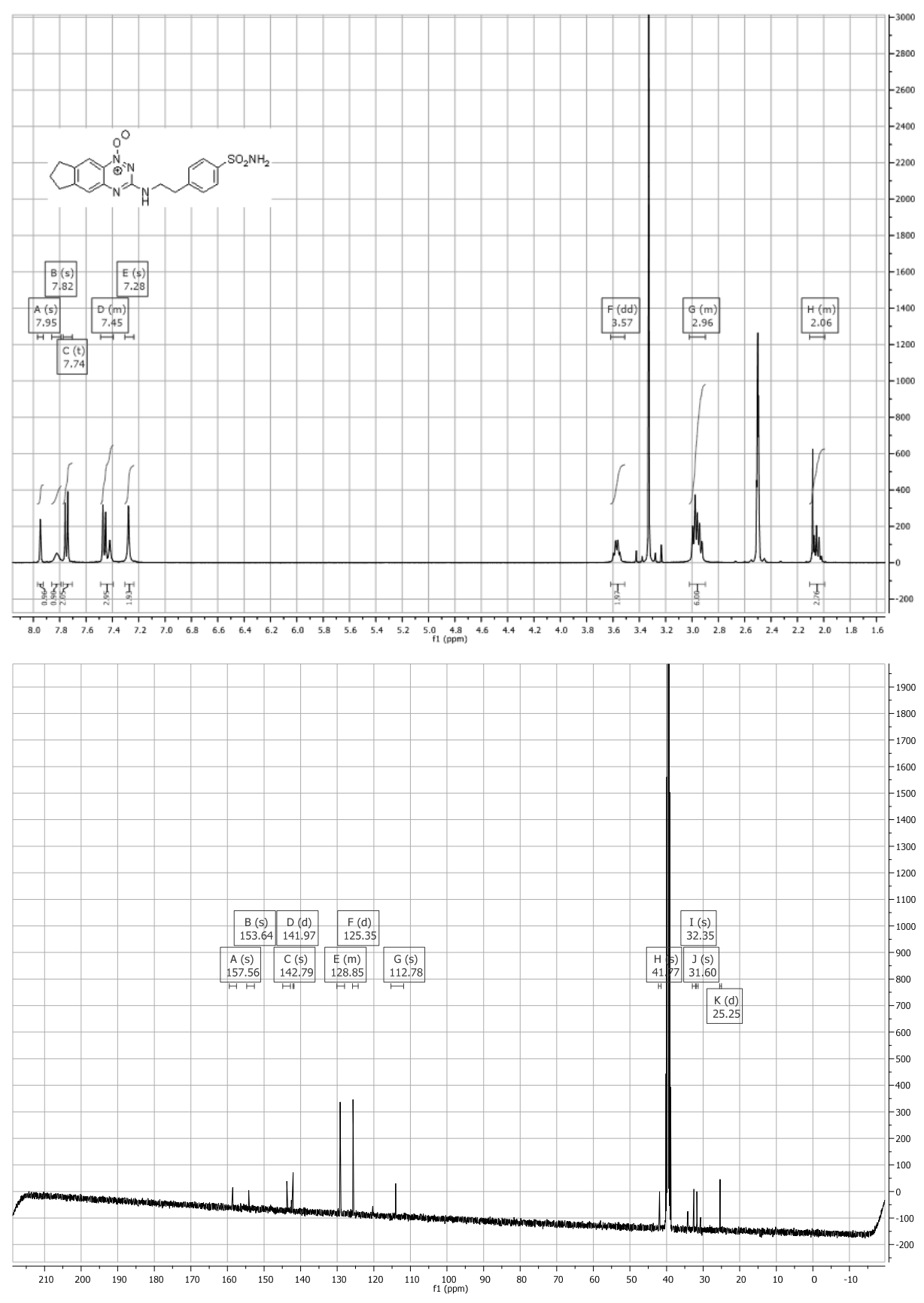

NMR spectra 9: 3-(4-sulfamoylphenethylamino)-7,8-dihydro-6H-indeno [5,6-e][1,2,4] triazine 1-oxide (10). 

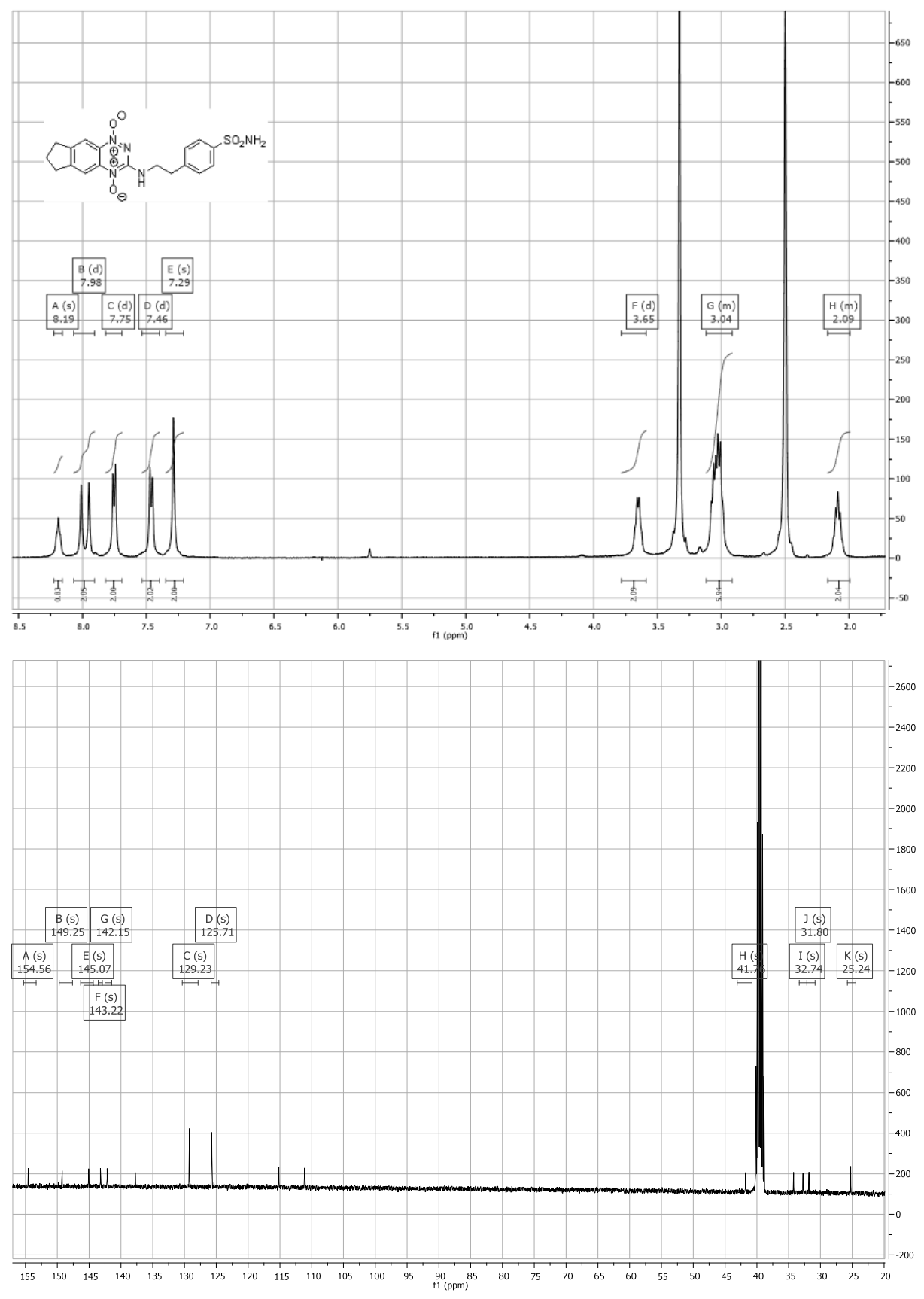

NMR spectra 10: 3-((4-sulfamoylphenethyl) amino)-7,8-dihydro-6H-indeno [5,6-e][1,2,4] triazine 1,4-dioxide (11). 
Chapter 5
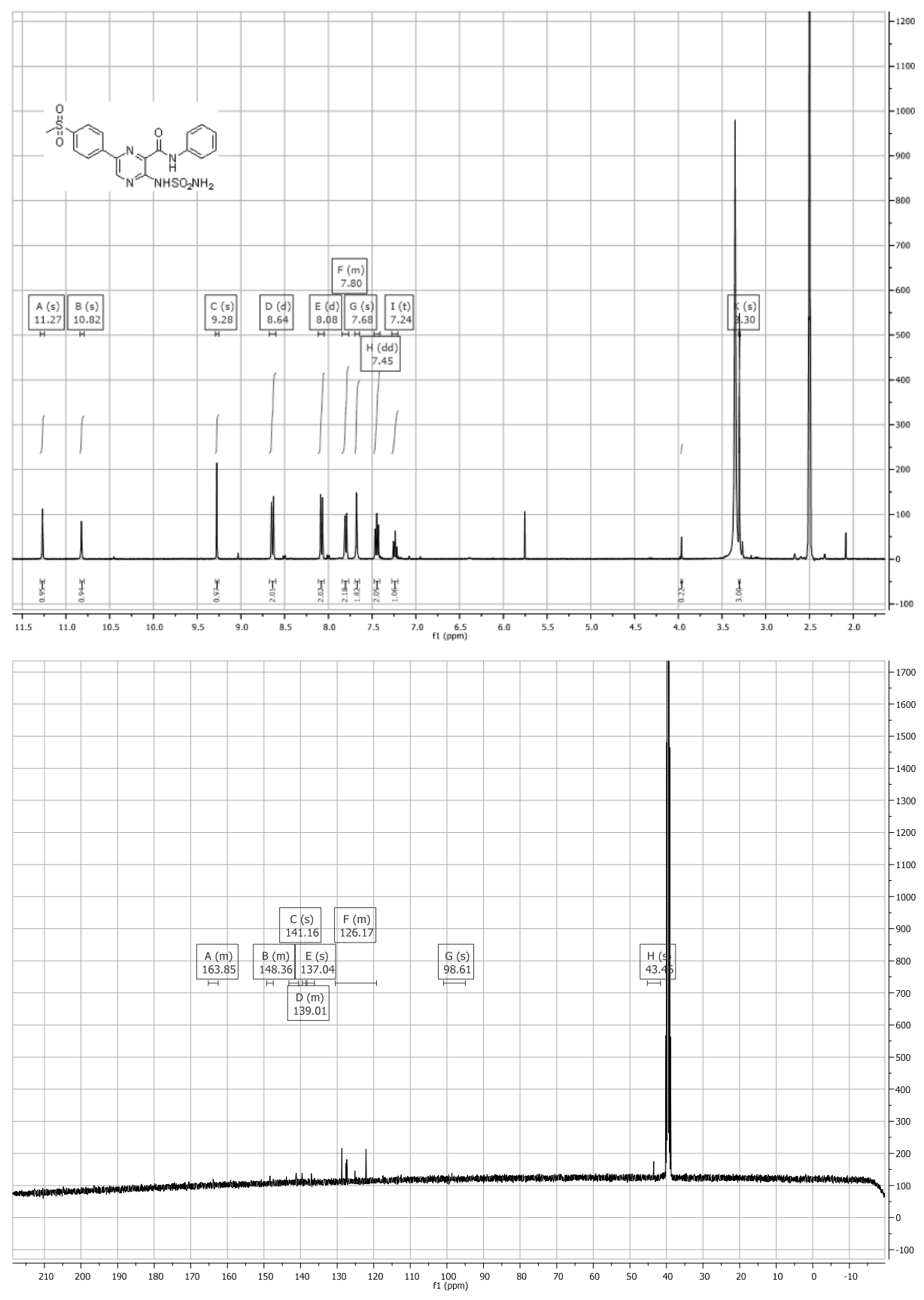

NMR spectra 11: 6-(4-(methylsulfonyl)phenyl)-N-phenyl-3-(sulfamoylamino)pyrazine-2-carboxamide (12). 

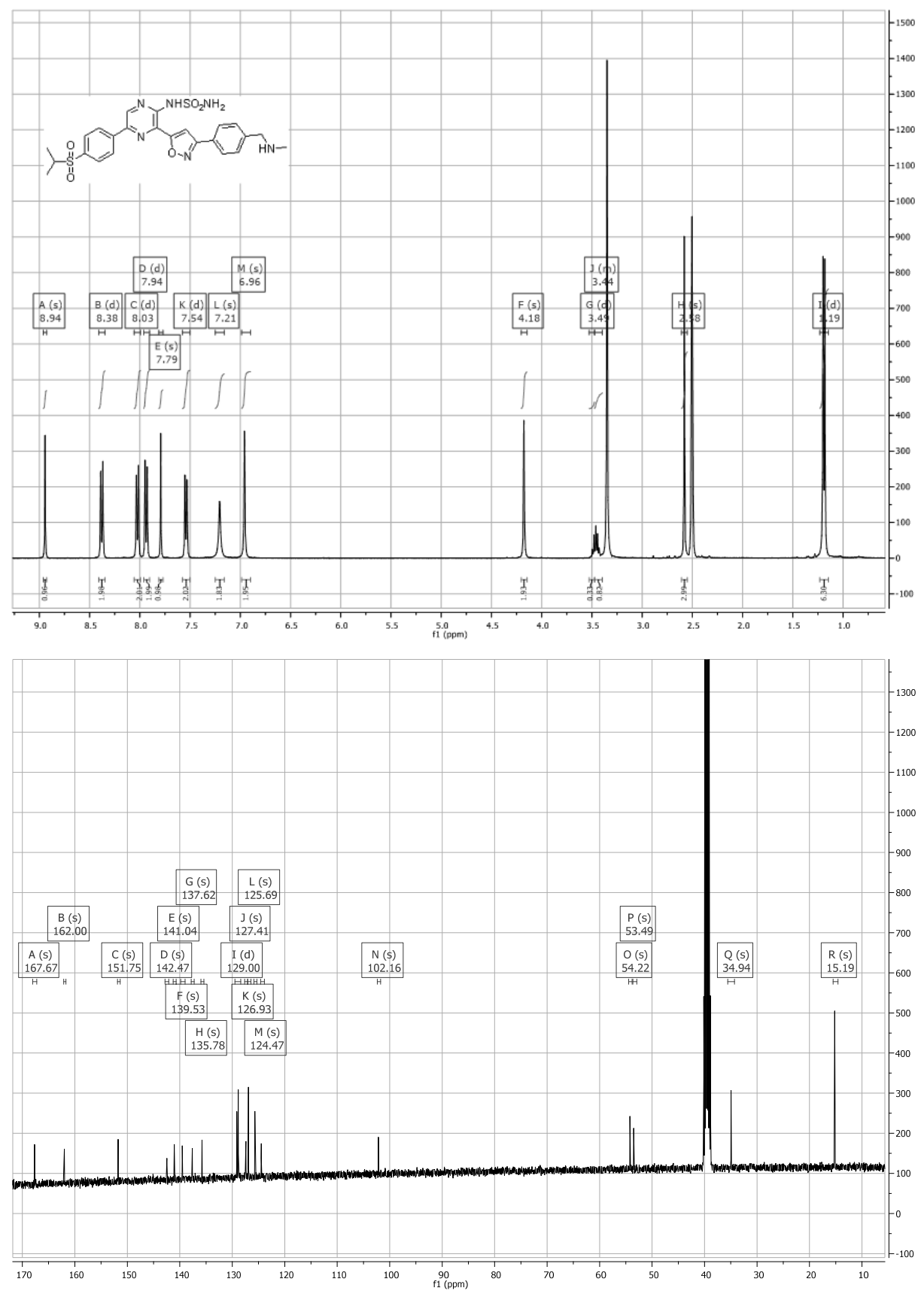

NMR spectra 12: 5-(4-(isopropylsulfonyl) phenyl)-3-(3-(4-((methylamino) methyl) phenyl) isoxazol-5-yl) pyrazin-2-carboxamide (13). 
Chapter 5
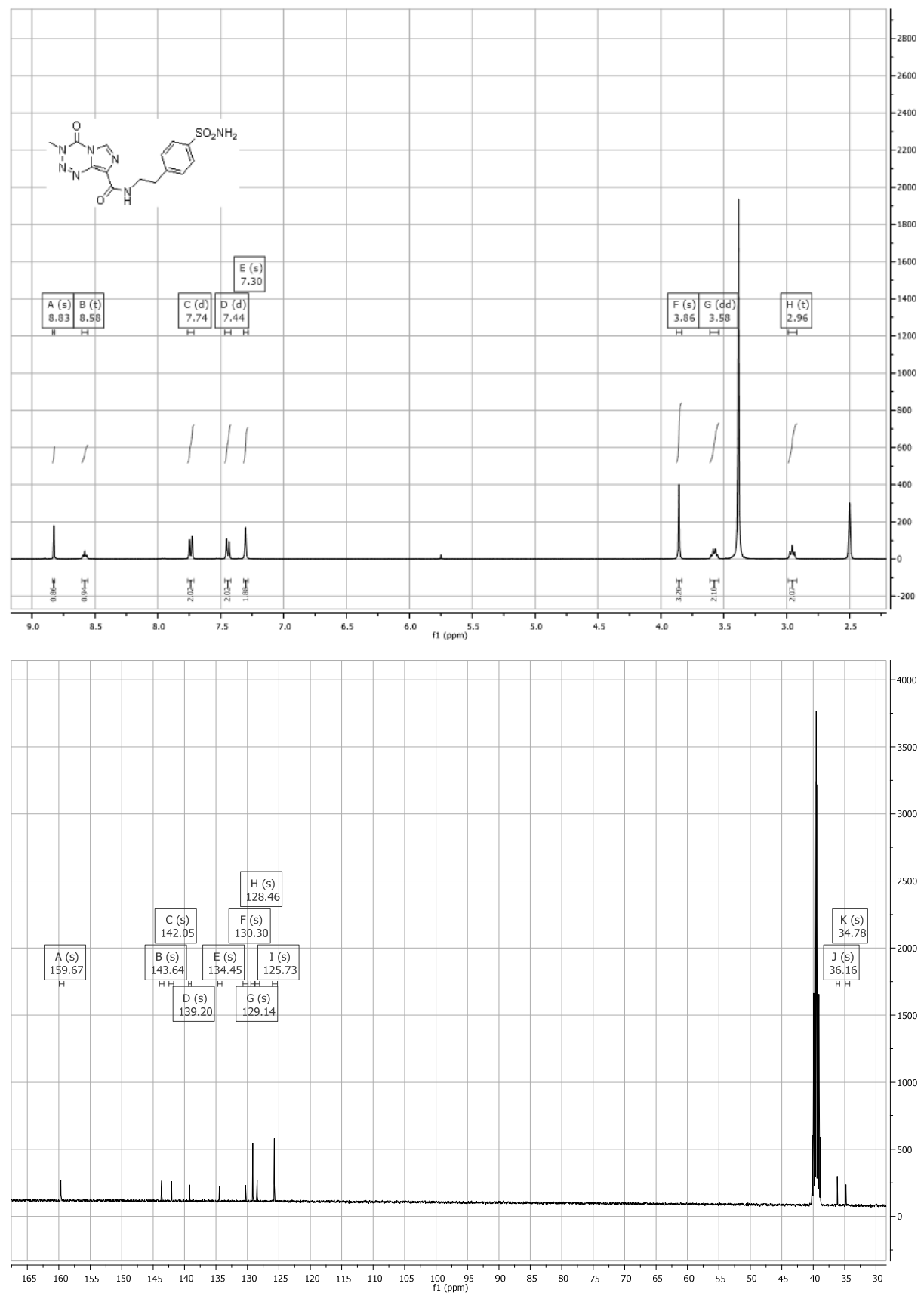

NMR spectra 13: 3-methyl-4-oxo-N-(4-sulfamoylphenethyl)-3,4-dihydroimidazo[5,1-d][1,2,3,5]tetrazine-8carboxamide (15a). 

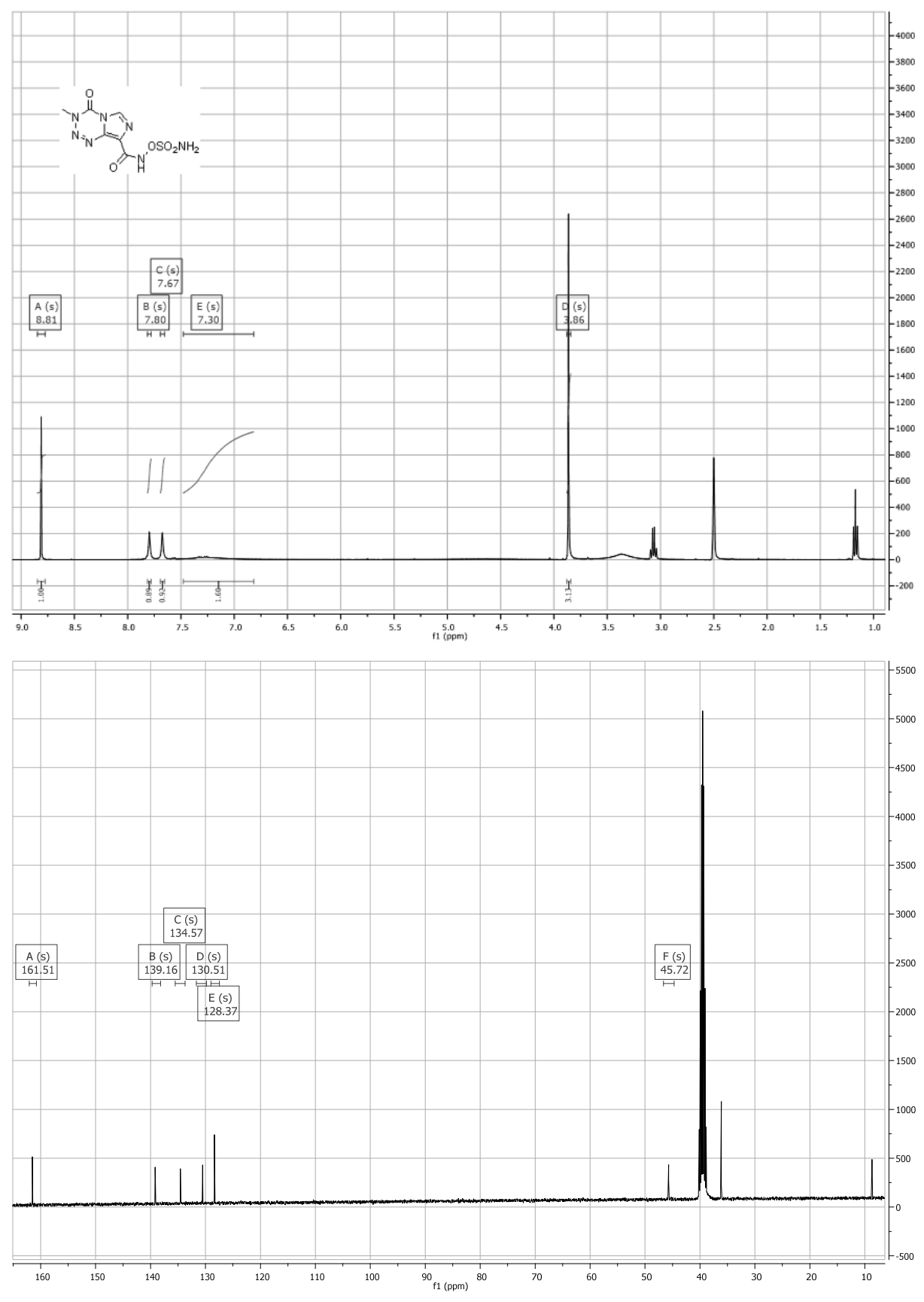

NMR spectra 14: 3-methyl-4-oxo-N-(sulfamoyloxy)-3,4-dihydroimidazo[5,1-d][1,2,3,5]tetrazine-8-carboxamide (15b). 

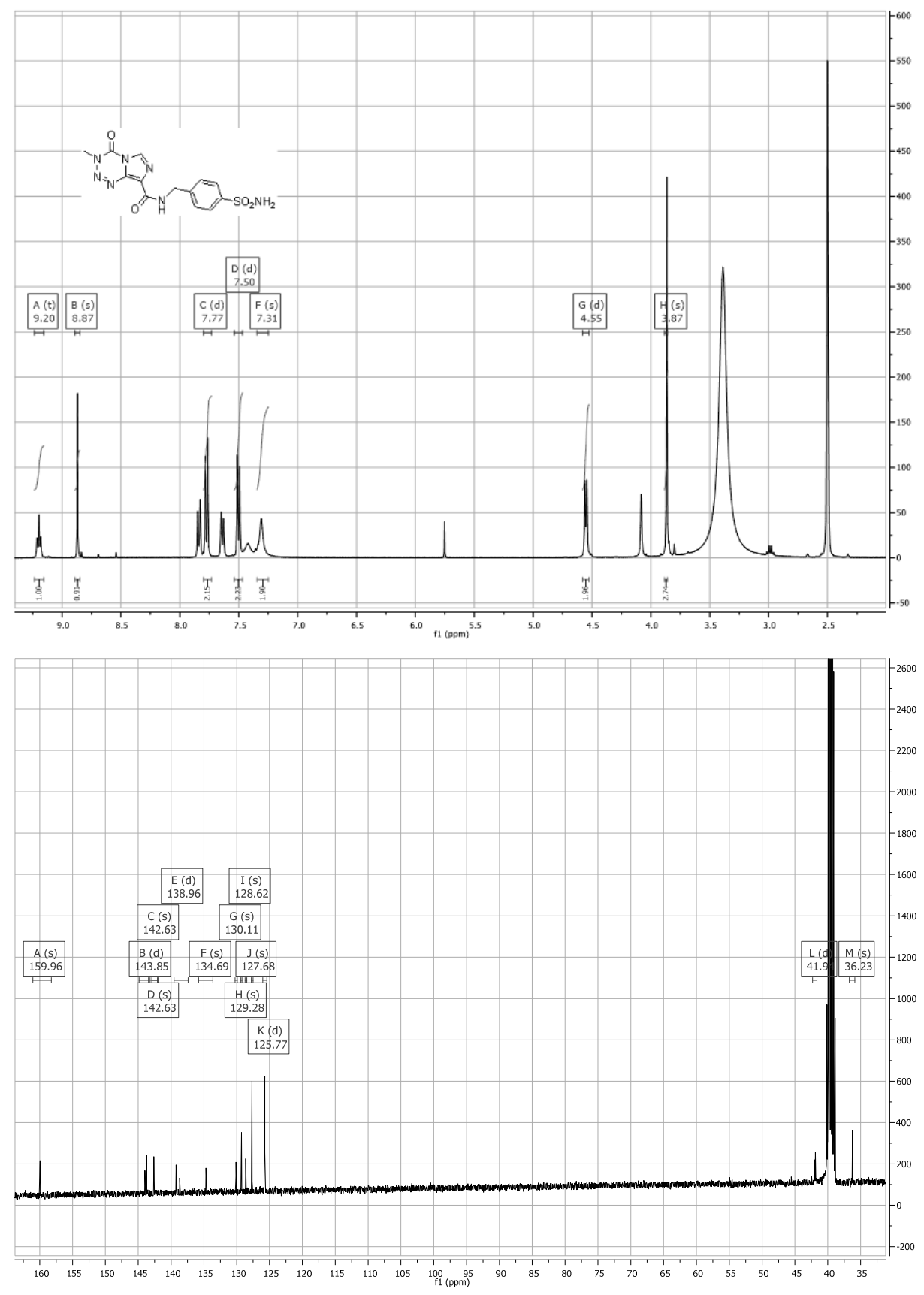

NMR spectra 15: 3-methyl-4-oxo-N-(4-sulfamoylbenzyl)-3,4-dihydroimidazo[5,1-d][1,2,3,5]tetrazine-8carboxamide (15c). 

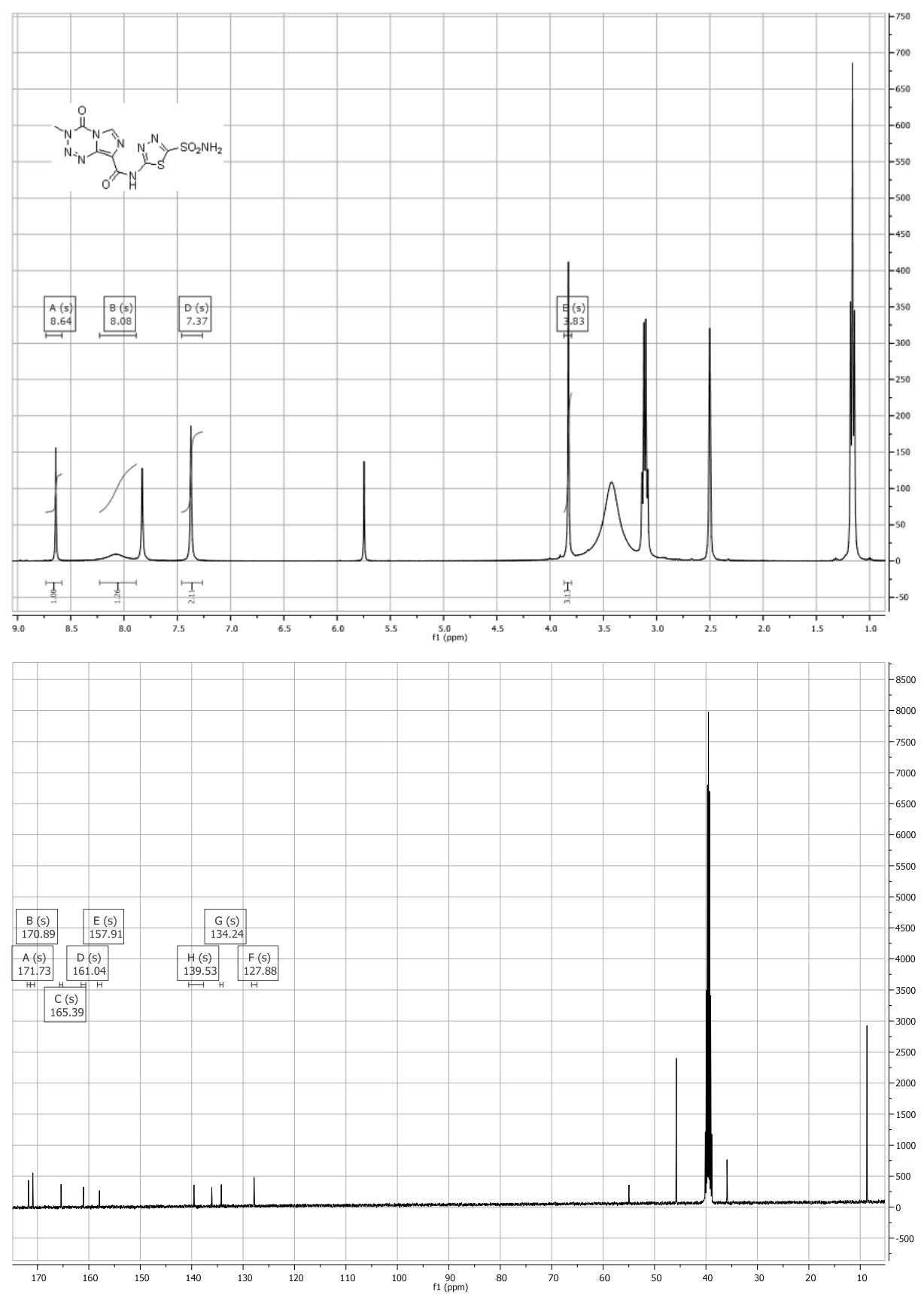

NMR spectra 16: 3-methyl-4-oxo-N-(5-sulfamoyl-1,3,4-thiadiazol-2-yl)-3,4-dihydroimidazo[5,1-d][1,2,3,5]tetrazine-8-carboxamide (15d). 

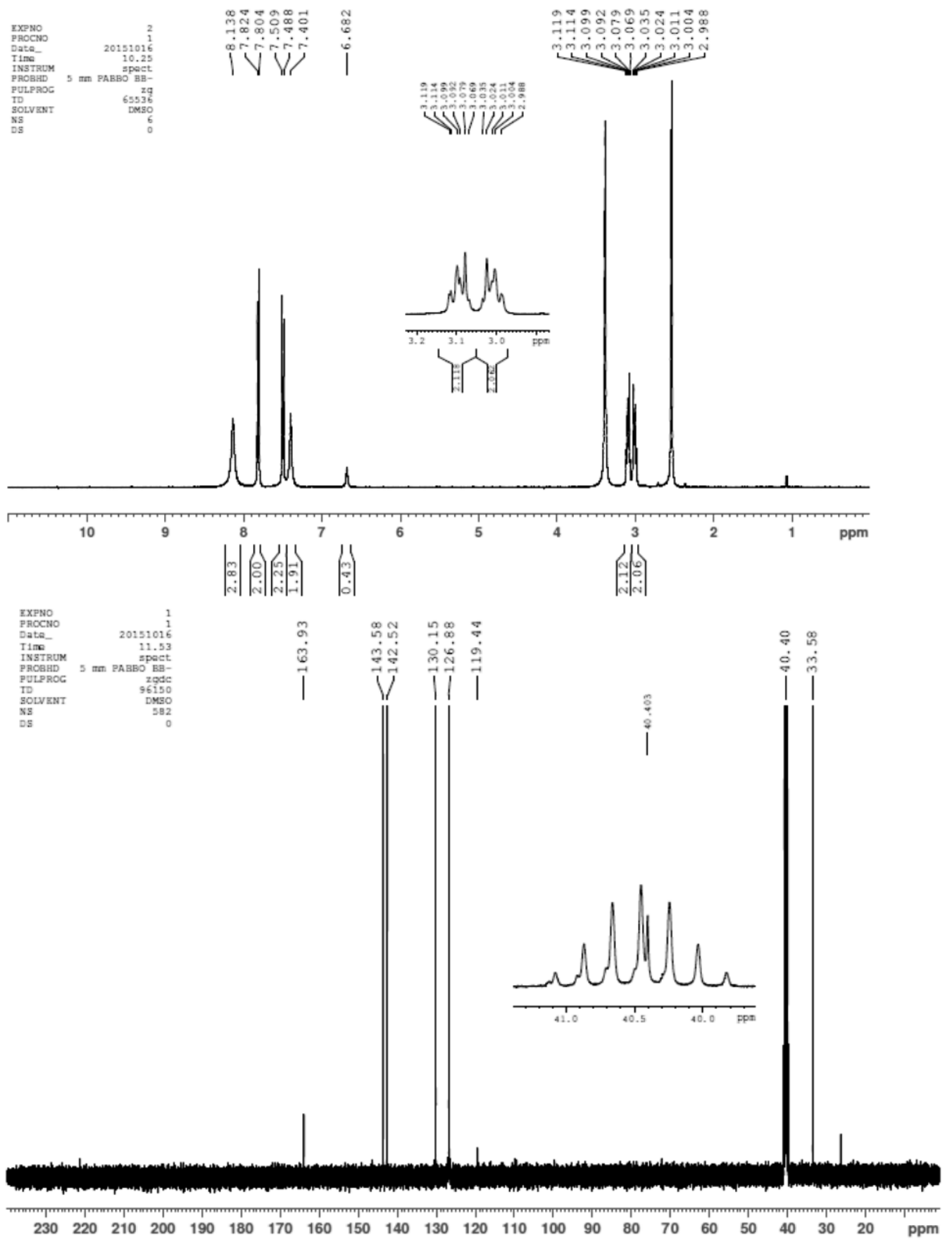

NMR spectra 17: 4-(2-(3-carbamimidoylguanidino)ethyl)benzenesulfonamide hydrochloride salt (18) 


\section{References}

1. Nordsmark, M., et al., Prognostic value of tumor oxygenation in 397 head and neck tumors after primary radiation therapy. An international multi-center study. Radiotherapy and oncology : journal of the European Society for Therapeutic Radiology and Oncology, 2005. 77(1): p. 18-24.

2. Hockel, M., et al., Association between tumor hypoxia and malignant progression in advanced cancer of the uterine cervix. Cancer research, 1996. 56(19): p. 4509-15.

3. Wojtkowiak, J.W., et al., Drug resistance and cellular adaptation to tumor acidic pH microenvironment. Molecular pharmaceutics, 2011. 8(6): p. 2032-8.

4. Good, J.S. and K.J. Harrington, The hallmarks of cancer and the radiation oncologist: updating the 5Rs of radiobiology. Clinical oncology, 2013. 25(10): p. 569-77.

5. Dubois, L.J., et al., New ways to image and target tumour hypoxia and its molecular responses. Radiotherapy and oncology : journal of the European Society for Therapeutic Radiology and Oncology, 2015. 116(3): p. 352-7.

6. Pettersen, E.O., et al., Targeting tumour hypoxia to prevent cancer metastasis. From biology, biosensing and technology to drug development: the METOXIA consortium. Journal of enzyme inhibition and medicinal chemistry, 2015. 30(5): p. 689-721.

7. Ebbesen, P., et al., Taking advantage of tumor cell adaptations to hypoxia for developing new tumor markers and treatment strategies. Journal of enzyme inhibition and medicinal chemistry, 2009. 24 Suppl 1: p. 1-39.

8. Neri, D. and C.T. Supuran, Interfering with $\mathrm{pH}$ regulation in tumours as a therapeutic strategy. Nature reviews. Drug discovery, 2011. 10(10): p. 767-77.

9. Pastorek, J. and S. Pastorekova, Hypoxia-induced carbonic anhydrase IX as a target for cancer therapy: from biology to clinical use. Seminars in cancer biology, 2015. 31: p. 52-64.

10. Pastorek, J., et al., Cloning and characterization of MN, a human tumor-associated protein with a domain homologous to carbonic anhydrase and a putative helix-loop-helix DNA binding segment. Oncogene, 1994. 9(10): p. 2877-88.

11. Wykoff, C.C., et al., Hypoxia-inducible expression of tumor-associated carbonic anhydrases. Cancer research, 2000. 60(24): p. 7075-83.

12. Kaluz, S., et al., Lowered oxygen tension induces expression of the hypoxia marker MN/carbonic anhydrase $I X$ in the absence of hypoxia-inducible factor 1 alpha stabilization: a role for phosphatidylinositol 3'-kinase. Cancer research, 2002. 62(15): p. 4469-77.

13. van den Beucken, T., et al., Hypoxia-induced expression of carbonic anhydrase 9 is dependent on the unfolded protein response. The Journal of biological chemistry, 2009. 284(36): p. 24204-12.

14. Kopacek, J., et al., MAPK pathway contributes to density-and hypoxia-induced expression of the tumorassociated carbonic anhydrase IX. Biochimica et biophysica acta, 2005. 1729(1): p. 41-9.

15. van Kuijk, S.J., et al., Prognostic Significance of Carbonic Anhydrase IX Expression in Cancer Patients: $A$ Meta-Analysis. Frontiers in oncology, 2016. 6: p. 69.

16. Peeters, S.G., et al., [(18)F]VM4-037 MicroPET Imaging and Biodistribution of Two In Vivo CAIXExpressing Tumor Models. Molecular imaging and biology : MIB : the official publication of the Academy of Molecular Imaging, 2015. 17(5): p. 615-9.

17. Akurathi, V., et al., Development and biological evaluation of (9)(9)mTc-sulfonamide derivatives for in vivo visualization of CA IX as surrogate tumor hypoxia markers. European journal of medicinal chemistry, 2014. 71: p. 374-84.

18. Akurathi, V., et al., Synthesis and biological evaluation of a 99mTc-labelled sulfonamide conjugate for in vivo visualization of carbonic anhydrase IX expression in tumor hypoxia. Nuclear medicine and biology, 2010. 37(5): p. 557-64.

19. Sneddon, D., et al., Synthesis and in Vivo Biological Evaluation of 68Ga-Labeled Carbonic Anhydrase IX Targeting Small Molecules for Positron Emission Tomography. Journal of medicinal chemistry, 2016. 


\section{Chapter 5}

20. Monti, S.M., C.T. Supuran, and G. De Simone, Anticancer carbonic anhydrase inhibitors: a patent review (2008 - 2013). Expert opinion on therapeutic patents, 2013. 23(6): p. 737-49.

21. Gieling, R.G., et al., Antimetastatic effect of sulfamate carbonic anhydrase IX inhibitors in breast carcinoma xenografts. Journal of medicinal chemistry, 2012. 55(11): p. 5591-600.

22. Ward, C., et al., Evaluation of carbonic anhydrase IX as a therapeutic target for inhibition of breast cancer invasion and metastasis using a series of in vitro breast cancer models. Oncotarget, 2015. 6(28): p. 24856-70.

23. Lock, F.E., et al., Targeting carbonic anhydrase IX depletes breast cancer stem cells within the hypoxic niche. Oncogene, 2013. 32(44): p. 5210-9.

24. Dubois, L., et al., Specific inhibition of carbonic anhydrase IX activity enhances the in vivo therapeutic effect of tumor irradiation. Radiotherapy and oncology : journal of the European Society for Therapeutic Radiology and Oncology, 2011. 99(3): p. 424-31.

25. Rami, M., et al., Hypoxia-targeting carbonic anhydrase IX inhibitors by a new series of nitroimidazolesulfonamides/sulfamides/sulfamates. Journal of medicinal chemistry, 2013. 56(21): p. 8512-20.

26. McDonald, P.C., et al., Recent developments in targeting carbonic anhydrase IX for cancer therapeutics. Oncotarget, 2012. 3(1): p. 84-97.

27. Supuran, C.T., Carbonic anhydrases: novel therapeutic applications for inhibitors and activators. Nature reviews. Drug discovery, 2008. 7(2): p. 168-81.

28. Dubois, L., et al., Targeting carbonic anhydrase IX by nitroimidazole based sulfamides enhances the therapeutic effect of tumor irradiation: a new concept of dual targeting drugs. Radiotherapy and oncology : journal of the European Society for Therapeutic Radiology and Oncology, 2013. 108(3): p. 523-8.

29. Barman, S., et al., Coumarin-benzothiazole-chlorambucil (Cou-Benz-Cbl) conjugate: an ESIPT based pH sensitive photoresponsive drug delivery system. Journal of Materials Chemistry B, 2015. 3(17): p. 349097.

30. Bekele, T., et al., Catalytic, enantioselective [4 + 2]-cycloadditions of ketene enolates and o-quinones: efficient entry to chiral, alpha-oxygenated carboxylic acid derivatives. Journal of the American Chemical Society, 2006. 128(6): p. 1810-1.

31. Gill, M. and A.F. Smrdel, Pigments of fungi, part 16. Synthesis of methyl (R)-(+)-tetrahydro-2-methyl-5oxo-2-furanacetate and its (S)-(-)-antipode, chiroptical references for determination of the absolute stereochemistry of fungal pre-anthraquinones. Tetrahedron Asymmetry, 1990. 1(7): p. 453-64.

32. Deshmukh, A.R. and V. Gumaste, Process for preparing alkyl/aryl chloroformates, 2005, Google Patents.

33. Hay, M.P., et al., Tricyclic [1,2,4]triazine 1,4-dioxides as hypoxia selective cytotoxins. Journal of medicinal chemistry, 2008. 51(21): p. 6853-65.

34. Arrowsmith, J., et al., Antitumor imidazotetrazines. 41. Conjugation of the antitumor agents mitozolomide and temozolomide to peptides and lexitropsins bearing DNA major and minor groovebinding structural motifs. Journal of medicinal chemistry, 2002. 45(25): p. 5458-70.

35. Ombouma, J., et al., Carbonic Anhydrase Glycoinhibitors belonging to the Aminoxysulfonamide Series. ACS medicinal chemistry letters, 2015. 6(7): p. 819-21.

36. Kelarev, V.I., et al., Synthesis of N-substituted 6-alkyl-2,4-diamino-1,3,5-triazines containing long alkyl radicals. Zhurnal Organicheskoi Khimii, 1988. 24: p. 1100-1105.

37. Svastova, E., et al., Carbonic anhydrase IX reduces E-cadherin-mediated adhesion of MDCK cells via interaction with beta-catenin. Experimental cell research, 2003. 290(2): p. 332-45.

38. Goede, V., et al., Past, present and future role of chlorambucil in the treatment of chronic lymphocytic leukemia. Leukemia \& lymphoma, 2015. 56(6): p. 1585-92.

39. Horsman, M.R. and P. Vaupel, Pathophysiological Basis for the Formation of the Tumor Microenvironment. Frontiers in oncology, 2016. 6: p. 66.

40. Reddy, S.B. and S.K. Williamson, Tirapazamine: a novel agent targeting hypoxic tumor cells. Expert opinion on investigational drugs, 2009. 18(1): p. 77-87.

41. Omuro, A. and L.M. DeAngelis, Glioblastoma and other malignant gliomas: a clinical review. JAMA, 2013. 310(17): p. 1842-50. 
42. Fukushima, T., H. Takeshima, and H. Kataoka, Anti-glioma therapy with temozolomide and status of the DNA-repair gene MGMT. Anticancer research, 2009. 29(11): p. 4845-54.

43. Dubois, L., et al., Imaging the hypoxia surrogate marker CA IX requires expression and catalytic activity for binding fluorescent sulfonamide inhibitors. Radiotherapy and oncology : journal of the European Society for Therapeutic Radiology and Oncology, 2007. 83(3): p. 367-73.

44. Ditte, P., et al., Phosphorylation of carbonic anhydrase IX controls its ability to mediate extracellular acidification in hypoxic tumors. Cancer research, 2011. 71(24): p. 7558-67.

45. Dubois, L., et al., Imaging of CA IX with fluorescent labelled sulfonamides distinguishes hypoxic and (re)oxygenated cells in a xenograft tumour model. Radiotherapy and oncology : journal of the European Society for Therapeutic Radiology and Oncology, 2009. 92(3): p. 423-8.

46. Fokas, E., et al., Targeting ATR in vivo using the novel inhibitor VE-822 results in selective sensitization of pancreatic tumors to radiation. Cell death \& disease, 2012. 3: p. e441.

47. Josse, R., et al., ATR inhibitors VE-821 and VX-970 sensitize cancer cells to topoisomerase $i$ inhibitors by disabling DNA replication initiation and fork elongation responses. Cancer research, 2014. 74(23): p. 6968-79.

48. Hall, A.B., et al., Potentiation of tumor responses to DNA damaging therapy by the selective ATR inhibitor VX-970. Oncotarget, 2014. 5(14): p. 5674-85.

49. Gray, L.H., et al., The concentration of oxygen dissolved in tissues at the time of irradiation as a factor in radiotherapy. The British journal of radiology, 1953. 26(312): p. 638-48.

50. Wright, E.A. and P. Howard-Flanders, The influence of oxygen on the radiosensitivity of mammalian tissues. Acta radiologica, 1957. 48(1): p. 26-32.

51. Doyen, J., et al., Knock-down of hypoxia-induced carbonic anhydrases IX and XII radiosensitizes tumor cells by increasing intracellular acidosis. Frontiers in oncology, 2012. 2: p. 199.

52. Kulshrestha, A., et al., Selective inhibition of tumor cell associated Vacuolar-ATPase 'a2' isoform overcomes cisplatin resistance in ovarian cancer cells. Molecular oncology, 2016.

53. De Milito, A. and S. Fais, Tumor acidity, chemoresistance and proton pump inhibitors. Future oncology, 2005. 1(6): p. 779-86.

54. Liao, C., et al., Genomic screening in vivo reveals the role played by vacuolar H+ ATPase and cytosolic acidification in sensitivity to DNA-damaging agents such as cisplatin. Molecular pharmacology, 2007. 71(2): p. 416-25.

55. van Gisbergen, M.W., et al., How do changes in the mtDNA and mitochondrial dysfunction influence cancer and cancer therapy? Challenges, opportunities and models. Mutation research. Reviews in mutation research, 2015. 764: p. 16-30.

56. Pollak, M., Potential applications for biguanides in oncology. The Journal of clinical investigation, 2013. 123(9): p. 3693-700.

57. Pernicova, I. and M. Korbonits, Metformin--mode of action and clinical implications for diabetes and cancer. Nature reviews. Endocrinology, 2014. 10(3): p. 143-56.

58. Liu, Z., et al., Phenformin Induces Cell Cycle Change, Apoptosis, and Mesenchymal-Epithelial Transition and Regulates the AMPK/mTOR/p70s6k and MAPK/ERK Pathways in Breast Cancer Cells. PloS one, 2015. 10(6): p. e0131207.

59. Shackelford, D.B., et al., LKB1 inactivation dictates therapeutic response of non-small cell lung cancer to the metabolism drug phenformin. Cancer cell, 2013. 23(2): p. 143-58.

60. Krall, N., et al., A small-molecule drug conjugate for the treatment of carbonic anhydrase IX expressing tumors. Angewandte Chemie, 2014. 53(16): p. 4231-5.

61. Khalifah, R.G., The carbon dioxide hydration activity of carbonic anhydrase. I. Stop-flow kinetic studies on the native human isoenzymes B and C. The Journal of biological chemistry, 1971. 246(8): p. 2561-73.

62. McIntyre, A., et al., Carbonic anhydrase IX promotes tumor growth and necrosis in vivo and inhibition enhances anti-VEGF therapy. Clinical cancer research : an official journal of the American Association for Cancer Research, 2012. 18(11): p. 3100-11. 



\section{Chapter}

\section{Nitroglycerin's influence on tumor hypoxia and perfusion in non-small cell lung cancer: a window-of-opportunity trial}

Submitted

Bart Reymen*, Marike W. van Gisbergen*, Aniek J.G. Even, Catharina M.L. Zegers, Marco Das, Erik Vegt, Joachim E. Wilberger, Felix Mottaghy, Ala Yaromina, Wouter van Elmpt, Dirk De Ruysscher, Ludwig Dubois, Philippe Lambin

* contributed equally 


\section{Abstract}

Despite preclinical evidence of nitric oxide donors influencing both tumor perfusion and hypoxia, they have not been shown in trials to improve the treatment results of all patients with non-small cell lung cancer. Biomarkers are therefore needed to select patients for treatment with NO donors. In this window-of-opportunity study nitroglycerin is shown to reduce uptake of the hypoxia-PET tracer HX4 in approximately two thirds of patients with hypoxic loco-regional disease. In a quarter of patients, hypoxic tumors and nodes become normoxic after treatment with nitroglycerin. Dynamic contrast enhanced CT-scans demonstrate that this effect on hypoxic tracer uptake is negatively correlated with tumor perfusion in hypoxic tumors. Using in vitro experiments, we show that the metabolism of 2-nitroimidazoles is not influenced by nitroglycerin itself and that nitroglycerin does not inhibit mitochondrial respiration at in human achievable concentrations. Therefore, 2-nitroimidazole based imaging biomarkers can be used to evaluate the nitroglycerin treatment efficacy in an individual patient. These results support the hypothesis that hypoxia scans and/or DCE-CT scans could form a tool to select patients for a nitroglycerin patch adjuvant to anti-cancer treatment (radiotherapy, chemotherapy, targeted agents or immunotherapy). An animation summarizing our results is available at https://youtu.be/udJSBYaRv9w. 


\section{Introduction}

Inspired by numerous publications highlighting the different biological effects of nitric oxide (NO) on cancer cells in the past decades, NO donors have been recognized as a potentially valuable -and mostly cheap- addition to current standard treatment strategies. Different clinical trials, some of them randomized, have explored the addition of NO donors to standard treatment in various cancer types. However, the obtained results are inconsistent. Some trials report favorable results for different cancer types, while others are not able to replicate these results and even mention the possibility of a detrimental effect in some patients [1-4].

Of all proposed mechanisms of action attributed to NO donors, their potential influence on cancer cell hypoxia is among the most widely cited $[3,5]$. There are different ways in which the oxygen concentration in cancer cells could be influenced by NO. Firstly, as a vasodilating agent, NO could enhance the blood flow and thereby increase oxygen transfer to the tumor. Secondly, NO has been shown to influence blood cell rheology, normalizing the blood viscosity and thereby improving the flux of blood cells through the aberrant vasculature of the tumor. Thirdly, blocking of the mitochondrial respiration and in this manner decreasing oxygen consumption [6-9]. However, there are concerns of NO donors eliciting the opposite effect by inducing steal effects caused by systemic vasodilatation, thereby actually decreasing tumor perfusion [10]. This makes the effect of NO donors in an individual patient difficult to predict.

The development of predictive biomarkers would enable an individualized approach of the adjuvant use of nitroglycerin to standard treatment for non-small cell lung cancer (NSCLC) [11]. In a window-of-opportunity trial design [12], we tested whether the effect of the NO donor nitroglycerin on the levels of hypoxia in an individual patient's tumor could be visualized using the 2-nitroimidazole positron emission tomography (PET) tracer $\left[{ }^{18} \mathrm{~F}\right]-\mathrm{HX} 4$ while measuring the perfusion effect of nitroglycerin using dynamic contrast-enhanced CT (DCE-CT) scans [13-18]. Secondly, because it is unclear whether the NO donor itself could affect the intracellular metabolism of 2-nitroimidazoles, and hereby lead to false positive results on hypoxia scans, we verified this in a separate in vitro experiment. Also, we assessed whether nitroglycerin had any effects on mitochondrial respiration in these cultures as this has been previously stated by other authors. Finally, this work should validate a potential imaging biomarker for future trials with nitroglycerin $[11,19]$. 


\section{Results}

\section{Inclusion and scans performed}

Forty-two patients were included in the trial before inclusion was halted in July 2016. The current analysis comprises the final analysis of all DCE-CT and HX4 PET scans performed (Table S1). Thirty-two patients received a baseline HX4 scan, of whom 25 also had an HX4 scan after application of a nitroglycerin patch. Twenty-eight patients received a baseline DCE-CT. After confirmation of the quality and timing of the DCE-CT, 22 scans were adequate for analysis. Eighteen patients underwent a DCE-CT after nitroglycerin of which 13 were suitable for analysis. For 12 patients, all scans (both baseline and post-nitroglycerin) were available for analysis. Twenty patients had both a baseline HX4 scan and a baseline DCE-CT scan (Table S2). The median interval between the baseline and second HX4 and DCE-CT scans was 4 and 5 days respectively (range 2-7 days). None of the patients received any cancer treatment in the interval between both scans.

All data is publicly available at http://www.cancerdata.org [20].

\section{Hypoxia is present at baseline in $>50 \%$ of NSCLC lesions and correlates with tumor volume and perfusion.}

In total 32 patients received a baseline HX4 scan (Table S1). Seventeen patients (53\%) exhibited a HX4 tumor to blood ratio (HX4-TBR) > 1.4 in the gross tumor volume of the primary tumor (GTVp) and 14 out of 25 patients (56\%) with pathologic lymph nodes had a HX4-TBR > 1.4 in the gross tumor volume of the lymph nodes (GTVIn). In total 22/32 patients (69\%) showed hypoxia in primary tumor and/or lymph nodes. Hypoxic tumors were significantly larger than non-hypoxic tumors at a median volume of $72.9 \mathrm{vs} 11 \mathrm{~cm}^{3}$ $(p=0.006)$. Likewise, hypoxic nodal volumes were larger than non-hypoxic volumes: 58.2 vs $9.4 \mathrm{~cm}^{3}(p=0.021)$. There was a significant correlation between tumor volume and baseline HX4-TBR for both primary tumor and nodes $(r=0.599, p<0.001$ and $r=0.688$, $p<0.0001$ respectively).

In the 20 patients who had both baseline HX4 scans and DCE-CT scans, the baseline level of tumor hypoxia (HX4-TBR) did not correlate with baseline tumor blood flow ( $r=-$ $0.334, p=0.15)$ or blood volume $(r=-0.08, p=0.738)$. However, in the 11 hypoxic tumors a significant negative correlation was found between baseline HX4-TBR and tumor blood flow ( $r=-0.618, p=0.043$ ) and blood volume ( $r=-0.736, p=0.010)$ (Fig. 1). 
A

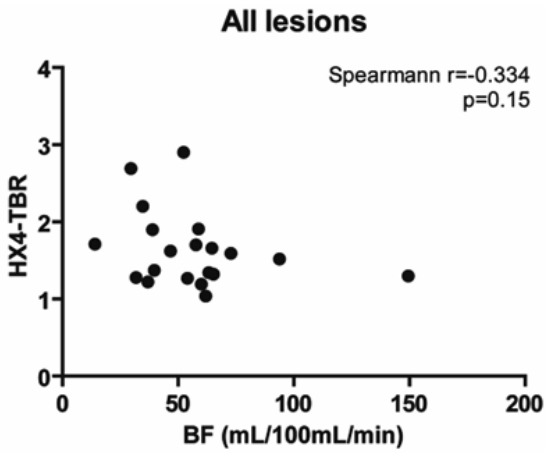

B

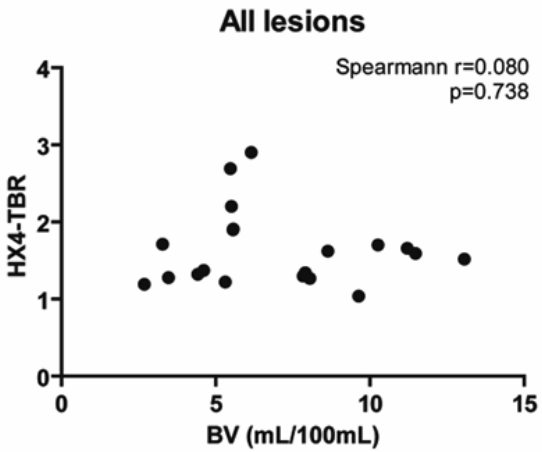

Hypoxic lesions

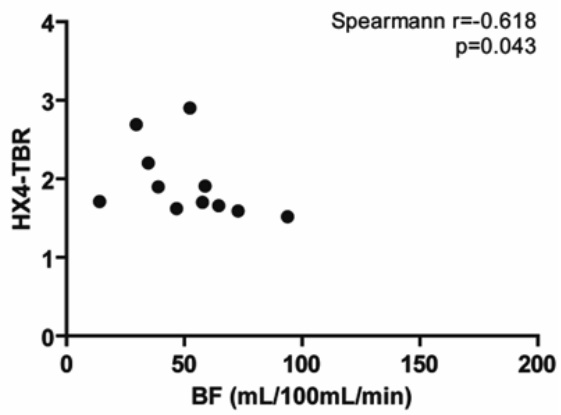

Hypoxic lesions

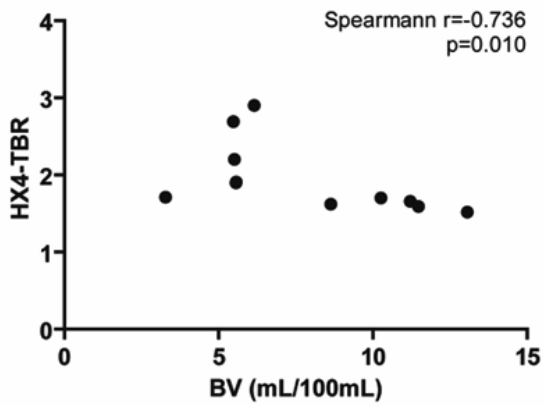

Figure 1. Correlation between blood volume, blood flow and HX4-TBR.

A. Negative correlation between tumor blood flow (BF) and HX4-TBR in hypoxic volumes. B. Negative correlation between tumor blood volume (BV) and HX4-TBR in hypoxic volumes.

\section{Nitroglycerin reduces $\mathrm{HX} 4$ uptake in a subset of NSCLC lesions}

We first evaluated the effect of nitroglycerin on HX4 uptake in the 22 patients with hypoxic volumes (Fig. 2A, Table 1). Fifteen of these patients, carrying a total of 19 hypoxic volumes (12 GTVp, $7 \mathrm{GTVIn}$ ), underwent a second HX4 scan. In 11 volumes (58\%) a decrease in the TBR was seen. After nitroglycerin administration, 4/15 patients (27\%) with baseline hypoxic volumes could not be classified as hypoxic anymore (Table 1).

To determine in individual patients whether this effect was larger than test-retest variability, we evaluated whether it exceeded the coefficient of repeatability (CoR): 17\% for the TBR and 7.8\% for the HX4-HF at a threshold for hypoxia of TBR>1.4 [17]. In this group of 19 hypoxic lesions, the HX4-HF dropped by $>7.8 \%$ in 4 volumes (21\%) belonging to 3 patients (Fig. 2B). The TBR decreased by $>17 \%$ in 4 volumes (21\%) in 3 patients, but also increased by $>17 \%$ in 4 volumes (21\%) belonging to 4 patients (Fig. 3A).

In the 12 non-hypoxic tumors and 11 nodal volumes, we noticed in 4 volumes (17\%) a rise of the TBR with $>17 \%$, while in 1 volume the TBR declined by $>17 \%$ (4\%). Three of 
the originally non-hypoxic primary tumors (25\%) and 1 nodal volume (9\%) became hypoxic (TBR>1.4) after the administration of nitroglycerin (Fig. 3B and Table 1).

Table 1. HX4 uptake parameters for non-hypoxic volumes (tumor and nodes pooled, $n=23$ ) and for hypoxic volumes (tumor and nodes pooled, $n=19$ ) before and after nitroglycerin patch (ie. only patients with both HX4-scans). Abbreviations: GTV= Gross Tumor Volume, SUV=Standardized Uptake Value, TBR= tumor-tobackground ratio of $\mathrm{HX} 4, \mathrm{HF}=$ high uptake fraction of $\mathrm{HX} 4, \mathrm{HV}=$ high-uptake volume of $\mathrm{HX} 4$.

\begin{tabular}{|c|c|c|c|c|c|}
\hline \multirow[t]{2}{*}{ Parameter } & \multicolumn{2}{|l|}{ Baseline } & \multicolumn{2}{|l|}{ Nitroglycerin } & \multirow{2}{*}{$\begin{array}{l}\text { Baseline vs } \\
\text { Nitroglycerin } \\
\text { p-value }\end{array}$} \\
\hline & Median \pm SD & Range & Median \pm SD & Range & \\
\hline \multicolumn{6}{|c|}{ Non-hypoxic volumes } \\
\hline $\operatorname{GTV}\left(\mathrm{cm}^{3}\right)$ & $9.6 \pm 12$ & $1.28-41.65$ & & & \\
\hline HX4-SUV mean & $0.63 \pm 0.17$ & $0.35-1.01$ & $0.64 \pm 0.17$ & $0.18-1.01$ & 0.984 \\
\hline HX4-SUV max & $0.86 \pm 0.25$ & $0.4-1.44$ & $0.92 \pm 0.28$ & $0.23-1.39$ & 0.911 \\
\hline HX4-TBR & $1.19 \pm 0.16$ & $0.639-1.37$ & $1.22 \pm 0.22$ & $0.64-1.66$ & 0.098 \\
\hline HX4-HF (\%) & 0 & 0 & $0 \pm 0.14$ & $0-6.6$ & 0.068 \\
\hline $\mathrm{HX} 4-\mathrm{HV}\left(\mathrm{cm}^{3}\right)$ & 0 & 0 & $0 \pm 0.06$ & $0-0.21$ & 0.068 \\
\hline \multicolumn{6}{|l|}{ Hypoxic volumes } \\
\hline $\operatorname{GTV}\left(\mathrm{cm}^{3}\right)$ & $56.98 \pm 70.53$ & $4.53-253.7$ & & & \\
\hline HX4-SUV mean & $0.67 \pm 0.22$ & $0.46-1.21$ & $0.74 \pm 0.22$ & $0.41-1.28$ & 0.276 \\
\hline HX4-SUV max & $1.26 \pm 0.36$ & $0.76-2.09$ & $1.25 \pm 0.5$ & $0.61-2.47$ & 0.507 \\
\hline HX4-TBR & $1.76 \pm 0.36$ & $1.42-2.69$ & $1.65 \pm 0.56$ & $1.17-2.91$ & 0.968 \\
\hline HX4-HF (\%) & $8 \pm 13.5$ & $0.06-40$ & $2 \pm 18.3$ & $0-55$ & 0.601 \\
\hline $\mathrm{HX} 4-\mathrm{HV}\left(\mathrm{cm}^{3}\right)$ & $3.1 \pm 15.3$ & $0.08-46$ & $1.5 \pm 11.4$ & $0-44.7$ & 0.314 \\
\hline
\end{tabular}

\section{Nitroglycerin`s effect on hypoxia correlates negatively with an effect on tumor perfusion in hypoxic tumors.}

Thirteen patients had both a baseline and a nitroglycerin DCE-CT scan. In this group of patients, neither median blood flow nor median blood volume changed significantly by application of a nitroglycerin patch ( $\mathrm{p}=0.507$ and 0.552 respectively). There was also no difference in response to nitroglycerin when evaluating the perfusion parameters between the groups of baseline hypoxic and non-hypoxic tumors (Table 2). On an individual patient level the blood flow increased in 5 (38\%) and blood volume in 7 tumors (54\%) (Fig. S1).

As expected there was a significant correlation between the change in blood flow and blood volume $(r=0.599, p=0.031)$. 
A

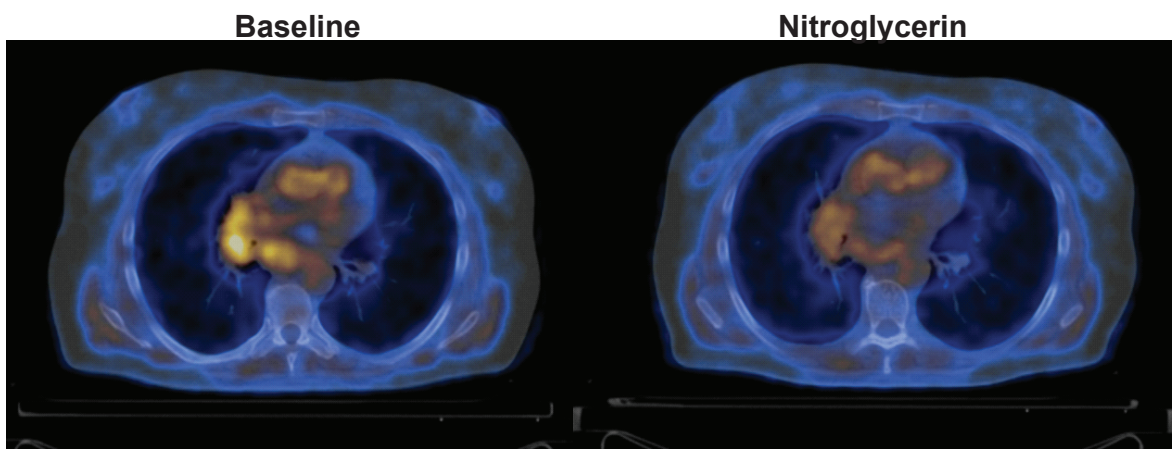

$\begin{array}{ll}\text { SUV: } 0.1 & \text { SUV: } 1.1\end{array}$

B

HF hypoxic lesions

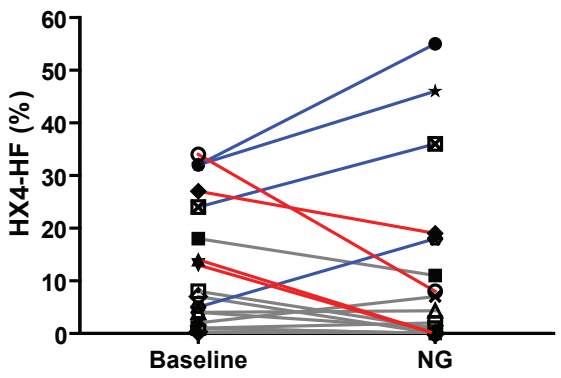

Figure 2. Uptake of $\mathrm{HX} 4$ before and after nitoglycerin administration.

A. Images of an HX4 PET/CT scan before and after nitroglycerin administration of the same patient. B. Evolution of HX4 high-uptake fraction (HX4-HF) after application of nitroglycerin patch in baseline hypoxic lesions. Red $=$ decline $>$ coefficient of repeatability $($ CoR) of HX4 (17\%), grey = stable: change $<$ CoR, blue $=$ rise $>$ CoR. $p=0.01$.

Twelve of these 13 patients also received a baseline and nitroglycerin HX4 scan. In 6 patients (50\%) the primary tumor was classified as hypoxic at baseline. In 4/6 patients with a baseline hypoxic tumor, nitroglycerin increased the tumor blood flow (range: 0.5$16.4 \mathrm{ml} / 100 \mathrm{ml} / \mathrm{min}$ ). In 3 out of these 4 patients, the HX4-TBR decreased (range: -0.1 to -0.43). In 2 of these patients the HX4-HF decreased (by $7 \%$ and $26 \%$ respectively), while rising in 2 (by $3 \%$ and $12 \%$ respectively).

In 4/6 hypoxic patients, the HX4-TBR decreased (range: -0.1 to -0.4) after application of a nitroglycerin patch. This led to a decreased high-uptake volume of HX4 (HX4-HV) in $3 / 6$ tumors (range: -0.2 to $-37.8 \mathrm{~cm}^{3}$ ) and in 2 patients, the decline in HX4-TBR exceeded the CoR of $17 \%$.

Conversely, in 5/6 patients with non-hypoxic tumors a decrease in the blood flow to the tumor was noticed (range: -0.6 to $-25.4 \mathrm{ml} / 100 \mathrm{ml} / \mathrm{min}$ ). In 3 of these tumors the HX4- 
TBR increased by $>17 \%$ (Fig. 3). In 2 patients the tumor became hypoxic after administration of nitroglycerin.

A

Hypoxic lesions

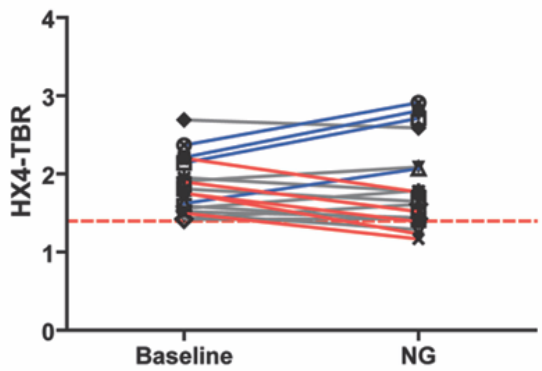

B

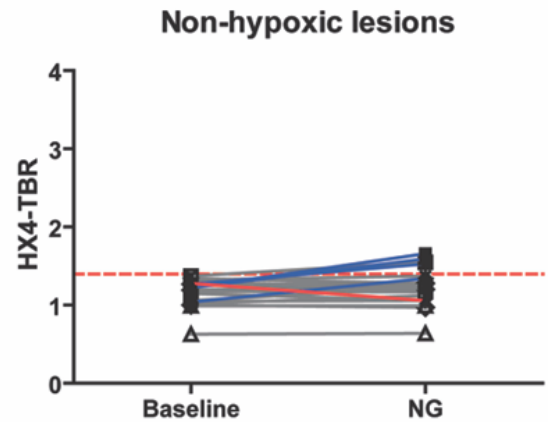

Figure 3. Tumor to background ratio (TBR) of HX4 after application of nitroglycerin patch.

A. Evolution of TBR of HX4 (HX4-TBR) after application of nitroglycerin patch in baseline hypoxic lesions, $p=0.052$ B. Evolution of HX4-TBR after application of nitroglycerin patch in non-hypoxic lesions, $p=0.551$. Red $=$ decline $>$ CoR of HX4 (17\%), grey = stable: change $<$ CoR, blue =rise $>$ CoR. Red striped line indicates cut-off for hypoxia: $H X 4-T B R=1.4$.

In the group of tumors with baseline hypoxia we found a significant negative correlation between the change in blood volume and HX4-HF ( $r=-0.829, p=0.042)$ and HX4-TBR ( $r=-$ 0.829, $p=0.042$, Fig. 4) and the change in HX4-HV ( $r=-1.000, p<0.0001)$. In non-hypoxic tumors there was no correlation between the HX4-TBR and either of the perfusion parameters.

Table 2. Perfusion parameters in patients with both a baseline DCE-CT scan and DCE-CT scan after nitroglycerin for non-hypoxic tumors $(n=6)$ and hypoxic tumors $(n=6)$

\begin{tabular}{|c|c|c|c|c|c|}
\hline \multirow[t]{2}{*}{ Parameter } & \multicolumn{2}{|l|}{ Baseline } & \multicolumn{2}{|l|}{ Nitroglycerin } & \multirow{2}{*}{$\begin{array}{l}\text { Baseline vs } \\
\text { Nitroglycerin } \\
\text { p-value }\end{array}$} \\
\hline & Median \pm SD & Range & Median \pm SD & Range & \\
\hline \multicolumn{6}{|l|}{ Non-hypoxic volumes } \\
\hline Blood Flow (ml/100ml/min) & $61 \pm 39.6$ & $36.1-149.5$ & $55.3 \pm 34.6$ & $28.8-126.7$ & 0.173 \\
\hline Blood Volume (ml/100ml) & $6.57 \pm 2.61$ & $2.69-9.64$ & $6.94 \pm 3.06$ & $2.39-9.59$ & 0.917 \\
\hline \multicolumn{6}{|l|}{ Hypoxic volumes } \\
\hline Blood Flow (ml/100ml/min) & $42.8 \pm 23.6$ & $29.6-93.8$ & $46.5 \pm 23.8$ & $30.1-93.4$ & 0.249 \\
\hline Blood Volume (ml/100ml) & $5.56 \pm 3.08$ & $5.48-13.06$ & $7.78 \pm 3.18$ & $5.36-1.41$ & 0.345 \\
\hline
\end{tabular}

Likewise, in the 6 tumors with the lowest blood flow (defined as tumors with a blood flow < median blood flow of $56 \mathrm{ml} / 100 \mathrm{ml} / \mathrm{min}$ ), significant negative correlations between the effect on blood flow and HX4-TBR ( $r=-0.943, p=0.005)$ and HX4-HF ( $r=-$ $0.943, p=0.005)$ and between the effect on blood volume and hypoxic volume $(r=-0.943$, 
$p=0.005)$ were found. In the 6 tumors with a blood flow $>$ median, these correlations were not present.
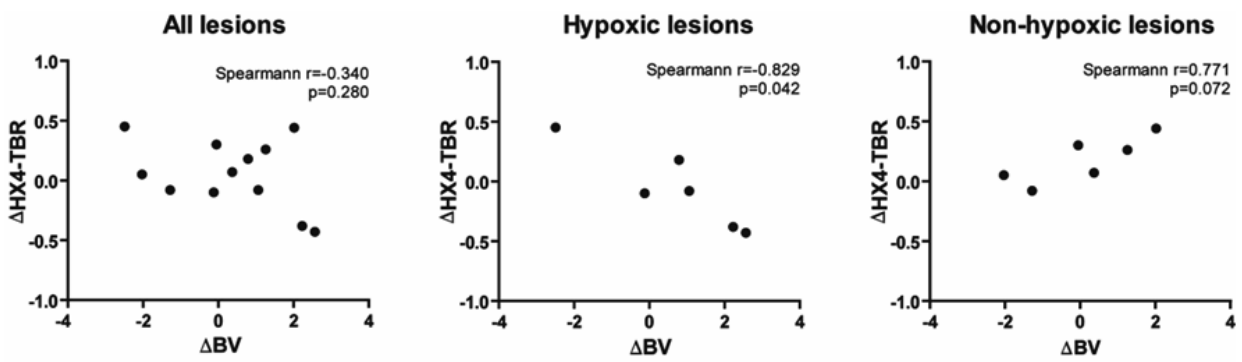

Figure 4. Negative correlation between nitroglycerin effect on blood volume $(\triangle B V)$ and $H X 4-T B R(\Delta H X 4-T B R)$.

Treatment with nitroglycerin at an in vivo achievable concentration does not reduce the oxygen consumption in vitro

In order to investigate if the decrease of the hypoxic fraction can be explained by the effect of NO on oxygen consumption by mitochondria, we investigated the potentially inhibitory effects of nitroglycerin on mitochondrial respiration. H522, H1299 and H1299 $\mathrm{Nfs} A$ cells were treated with either vehicle or nitroglycerin at concentrations of $352 \mathrm{pM}$, $352 \mathrm{nM}$ and $35.2 \mu \mathrm{M}$ as the respective $\mathrm{IC}_{50}$ concentrations could not be reached. These concentrations are based on the paper from Mc Allister, taking in account that our patch is 2 times smaller in size [21]. The concentration of $352 \mathrm{pM}$ is in the range of the plasma concentration. In all investigated cell lines nitroglycerin, did not cause a decline of the OCR percentage (Fig. 5A) at used concentrations. In order to make the cells more dependent on oxidative phosphorylation and thereby elicit more distinctive effects of nitroglycerin, cells were pre-exposed to galactose. However, also under these conditions, no differences could be observed (Fig. S2).

\section{Nitroglycerin reduction does not affect pimonidazole binding in vitro}

To assess if nitroglycerin affects tumor cell's reductase capacity, resulting in a decreased uptake of 2-nitroimidazoles, H1299 parental and nitroreductase overexpressing cells were exposed to pimonidazole under normoxic conditions. Pimonidazole reduction occurred only upon hypoxic exposure in H1299 parental cells, whereas a clear pimonidazole signal was detected upon normoxia in H1299 NfsA cells which was equally strong in cells exposed to hypoxia. Nitroglycerin, at the highest used concentration (35.2 mM), did not affect pimonidazole binding in any experimental condition (Fig. 5B). 
A

H522

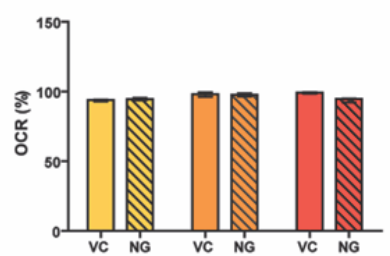

H1299

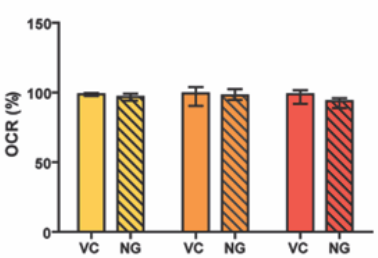

H1299 NfsA

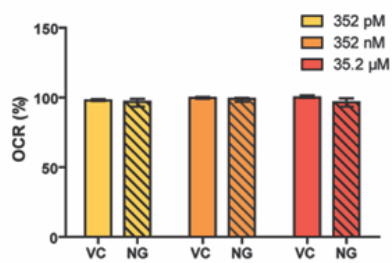

B

\section{H1299}

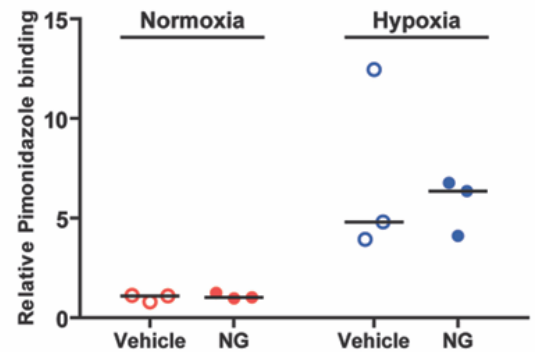

H1299 NfsA

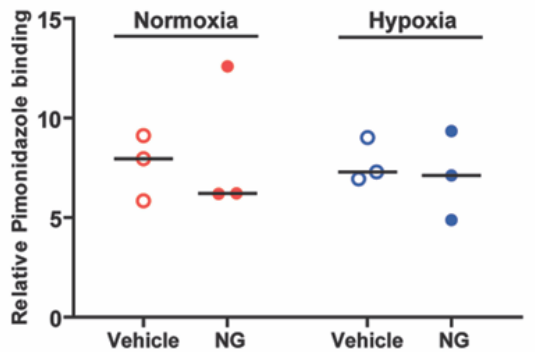

Figure 5. In vitro exposure of nitroglycerin does not lead to decreased cellular respiration or pimonidazole binding.

A. Nitroglycerin does not affect cellular respiration. Relative inhibition of mitochondrial respiration of H522 cells, H1299 or H1299 NfsA upon treatment with nitroglycerin (NG): 352 pM (plasma concentration); 352 nM; $35.2 \mu \mathrm{M}$ or vehicle control (VC). Measurements were normalized for protein concentration and the $\%$ of baseline oxygen consumption rate (OCR) before adding nitroglycerin or vehicle. Results are shown for median \pm range of at least 3 independent experiments. B. Upon nitroglycerin exposure, pimonidazole binding is not decreased under hypoxic conditions, neither under normoxic conditions for H1299 or H1299 NfsA cells. Data represents relative pimonidazole binding of 3 independent experiments

\section{Discussion}

In recent years, NO donors have attracted attention as possible anti-cancer agents, to be used either as a single agent or combined with conventional modalities such as chemotherapy and radiotherapy. Possible mechanisms of action proposed by different authors include enhanced tumor perfusion and chemotherapy delivery, anti-tumoral immunogenic effects, upregulation of p53 or radio-sensitization by either increased formation of intracellular free radicals, decreased tumor hypoxia via increasing perfusion and/or inhibition of the mitochondrial respiration [4, 9, 22-24]

Consequently, a number of clinical trials, without an up-front biomarker-based selection or stratification, have been conducted to study the effect of the addition of the NO donor nitroglycerin to standard therapy [5, 25-29]. Yasuda et al. were the first to 
perform a randomized phase II trial in which 120 patients with stage IIIB-IV NSCLC were randomized between 4 cycles of cisplatin-vinorelbin chemotherapy combined with a nitroglycerin $25 \mathrm{mg}$ patch or placebo. They found a significantly improved overall response rate, time-to-progression and overall survival in favor of patients treated with nitroglycerin, while treatment toxicity remained equal [5]. After this first positive trial with all Asian patients, Reinmuth et al. published a similar trial with 66 Caucasian patients with stage IIIB-IV NSCLC. Instead of a predetermined 4 cycles of cisplatinvinorelbin chemotherapy they continued treatment until disease progression. The trial was halted prematurely due to slow accrual and despite a numerically higher overall response rate in the nitroglycerin arm, the authors could not find any statistically significant survival benefit of the addition of nitroglycerin to standard chemotherapy [25]. The Dutch NVALT-12 trial randomized 223 patients with or without a $15 \mathrm{mg}$ nitroglycerin patch and chemotherapy treatment with paclitaxel-carboplatinbevacizumab (PCB). They too failed to demonstrate a statistically significant difference in overall and progression free survival between the 2 groups [27]. In a four-arm phase II-trial investigating irinotecan/cisplatin (IP) versus irinotecan/capecitabine (IX) with or without isosorbide-5-mononitrate (ISMN) in 146 patients with chemo-naïve stage IIIB-IV NSCLC, Han et al. also failed to prove that adding this NO-donor to chemotherapy had an impact on the overall response rate [26]. The Australasian NITRO trial randomizing patients with advanced NSCLC to platinum-based doublet chemotherapy with or without a $25 \mathrm{mg}$ patch of nitroglycerin was closed for accrual after an interim analysis showed an equal PFS, OS and ORR in both groups [28]. Lastly, Arrieta et al. published the results of a small phase II trial treating 35 patients with stage III NSCLC with 6 cycles of cisplatin-vinorelbin chemotherapy. Cycles 3 and 4 were given concurrently with radiotherapy to a median total dose of $60 \mathrm{~Gy}$ (54 Gy to the lymph nodes). A $25 \mathrm{mg}$ patch of nitroglycerin was applied in all patients for 5 days around each chemotherapy cycle and every day during radiotherapy. They achieved a median OS of 26.5 months, which is in line with the results of recent trials on concurrent chemo-radiation for stage III NSCLC [29]. We can therefore conclude that the addition of NO donors to standard therapy has not been shown to have a major impact on the survival of unselected patients with NSCLC. We hypothesize that a biomarker-based selection for additional treatment with nitroglycerin could increase the potential benefit for individual patients. Since a potential effect on tumor perfusion and hypoxia are most cited as mechanisms behind a treatment effect [30], we hypothesize that these could serve as key biomarkers to select patients for nitroglycerin treatment.

In our clinical study we observed a reduction of HX4 uptake of varying magnitude upon application of a nitroglycerin patch in approximately two thirds of patients with hypoxic loco-regional disease. In a quarter of patients with baseline hypoxia, the tumor and nodes become normoxic after treatment with nitroglycerin. One possible explanation for this observation could have been a blocking effect of NO-donors on the oxidative 
phosphorylation. Several authors have previously proposed this phenomenon as a possible radiosensitizing effect of NO-donors [31]. Such an inhibition reduces the amount of oxygen used for oxidative phosphorylation by the mitochondria, increasing the amount of intracellular oxygen and enhancing the possibility of free radical formation upon irradiation of the cell [8]. Our in vitro experiments in several NSCLC cell lines do not support an inhibitory effect of nitroglycerin on mitochondrial respiration under aerobic oxygen concentrations at in vivo achievable concentrations [32-34]. A normal plasma concentration of nitroglycerin 2-4 hours obtained after application of a Transiderm Nitro $5 \mathrm{mg}$ patch as used in our trial, is reported to be $0.16 \pm 0.03 \mu \mathrm{g} / \mathrm{l}$ (Product Monograph Transiderm Nitro, Novartis 2011). Even forcing the cellular metabolism to be more dependent on oxidative phosphorylation using galactose preincubation $[35,36]$ in order to elicit a more pronounced effect of NO release by nitroglycerin, did not result in decreased oxygen consumption (Fig. 4A, Fig. S2). Therefore, an acute effect of blocking the oxidative phosphorylation chain in human tumors with conventional doses of nitroglycerin seems unlikely.

To investigate the possibility that nitroglycerin itself affects reductase capacity of tumor cells as a mechanism explaining the decreased HX4 uptake, in vitro tests were carried out. The binding of pimonidazole was not affected upon nitroglycerin treatment under both normoxic and hypoxic conditions in parental and nitroreductase overexpressing cells, suggesting an extracellular mechanism of nitroglycerin, such as improved perfusion, to explain its effects on tumor hypoxia seen on HX4 PET imaging.

When studying the entire patient population however, the baseline uptake of HX4 and perfusion parameters were not correlated. Only in hypoxic tumors negative correlations between the baseline level of hypoxia (HX4-TBR and HX4-HF) and tumor perfusion (BF and BV) were found. Furthermore, in hypoxic tumors the effect of nitroglycerin on hypoxia was negatively correlated with an effect on perfusion, which was not present in non-hypoxic tumors.

This suggests that improved perfusion upon nitroglycerin treatment is at least in part responsible for the decreased $\mathrm{HX} 4$ uptake in certain lesions. However, the perfusion effects of nitroglycerin seem to be not strictly limited to a general enhancement of perfusion. Interestingly, an increased tumor blood flow after nitroglycerin treatment was almost exclusively found in patients with hypoxic tumors at baseline. The majority of non-hypoxic tumors showed various levels of decreased perfusion after application of a nitroglycerin patch. One in four non-hypoxic volumes became hypoxic after treatment with nitroglycerin. This observation correlates with warnings in literature that NO should be considered a "double edged sword" in cancer treatment [37]. As nitroglycerin is not a targeted agent focusing its actions solely on the tumor vasculature, its systemic effects on peripheral vessels could for instance cause a steal phenomenon in already well perfused tumors, shifting blood away from the tumor to the systemic vasculature [10, 
38]. Thus, nitroglycerin could serve as a regulator, enhancing therapeutic effects in poorly perfused tumors while having no or even a deleterious effect on well-perfused tumors. By performing window-of-opportunity trials aimed at the pre-selection of patients most likely to benefit, these potentially deleterious effects could possibly be minimized, while optimizing the chance of a positive effect for the individual patient [12].

When studying the uptake of fluorinated 2-nitroimidazoles, a point of concern is the definition of a meaningful variation in uptake. HX4 is a relatively new tracer which has been shown in a rat tumor model to have a favorable pharmacokinetic profile compared to other tracers. An adequate spatial reproducibility of HX4 uptake in rats has also been found earlier [39, 40]. Reproducibility of HX4 uptake in humans was also tested and a high stability of SUV, TBR, hypoxic volume and hypoxic fraction values was found. Applying the coefficient of repeatability of $17 \%$ for the HX4-TBR and $7.8 \%$ for the hypoxic fraction at a threshold of TBR>1.4 for hypoxia, we still see a meaningful decline in high hypoxic fraction in about one third of the hypoxic volumes. When looking at the CoR determined by Zegers et al, it should also be noted that this was a multicenter study with a median post-injection scan interval of only 99 minutes and short acquisition time per bed position, while we performed imaging at the optimal scanning time point at 4 hours for HX4 using a prolonged acquisition per bed position [41]. This means the CoR of $17 \%$ will most probably be an overestimation when compared to our current single-center, single scanner series with a median post-injection scan-time of 4 hours [17].

In our study, we found that smaller tumor volumes were less likely to be classified as hypoxic. Whether this is a true finding or one merely caused by the limited spatial resolution of PET-scanning is unknown. There were also only a limited number of patients who were able to undergo all HX4 and DCE-CT scans, which is due to logistic issues as well as patient acceptance of the burden of undergoing all scans. Since the above mentioned correlations between the effects of nitroglycerin on perfusion and hypoxia are based on a limited number of patients, these results must be interpreted with caution.

Variations in the published DCE-CT scanning techniques and considerable interobserver variability have been reported when analyzing perfusion parameters. Therefore, a cutoff for repeatability of the blood flow and blood volume parameters is difficult to establish from literature $[42,43]$. To at least make our results as consistent as possible, all of our DCE-CT scans were performed under supervision of an experienced radiologist $(M D)$, while one person ( $A E$ ) analyzed all the acquired scans. Rigorous quality control of the imaging parameters and timing before analysis further limited the final number of scans deemed suitable.

We also acknowledge the limitations of the in vitro studies. Investigating the interplay between mitochondrial oxygen consumption and a NO donor is difficult. While 
investigating the mitochondrial respiration upon nitroglycerin exposure we gave a single exposure of nitroglycerin solution, which does not correlate with the clinical situation in which a NO donor is administered continuously for hours every day. This is because our cell system is not optimized to give a low concentration in combination with a gradual exposure and clearance of the nitroglycerin. Additionally, for nitroglycerin often stabilizers in the form of saccharides are used which can bias effects on mitochondrial respiration. Concentrations of nitroglycerin (352 pM), corresponding with the observed plasma concentrations by McAllister et al. [21], exposure did not result in a decreased mitochondrial respiration in our experiments; even increasing the dose 100.000 fold to $35.2 \mu \mathrm{M}$ did not lead to a significant decrease of the oxygen consumption in cells. Additionally, due to the previously reported nitroglycerin tolerance, which already occurs after a time period of 3 hours of exposure, investigating long exposure of low nitroglycerin concentrations was not feasible [44].

In spite of the above mentioned limitations, we have demonstrated that in a subgroup of hypoxic volumes there is a meaningful effect of nitroglycerin on the uptake of the 2nitroimidazole HX4 in human NSCLC, which is not due to an artefact on the cellular metabolism of 2-nitroimidazoles or an effect on the mitochondrial oxygen consumption rate, but correlates with an effect on perfusion in a subgroup of patients. Moreover, this perfusion effect seems to vary depending on the baseline tumor perfusion and oxygenation status, which is a point of concern when designing and interpreting the results of clinical trials investigating nitroglycerin as an adjunct to standard treatment. The actual effect in terms of improvement of individual tumor control and survival remains to be determined in the analysis of clinical trial NCT01210378. Also, the influence of intra-tumor heterogeneity with respect to hypoxia and perfusion on the potential results of nitroglycerin treatment remains to be determined. The current results do support the hypothesis that hypoxia scans and/or DCE-CT scans could form a tool to select patients for treatment with a nitroglycerin patch adjuvant to anti-cancer treatment (radiotherapy, chemotherapy, targeted agents or immunotherapy) to increase drug uptake and/or decrease hypoxia. Since the current procedure of scanning and evaluating the effects of nitroglycerin in individual patients using multiple scans is burdensome and laborious, future research should also be aimed at identification of subjects using single scans, radiomics-based signature and/or blood biomarkers $[45,46]$. 


\section{Material and Methods}

\section{Clinical experiments}

Patient's characteristics and study design

Patients diagnosed with non-small cell lung cancer stage Ib-IV referred to Maastro Clinic for radical radiotherapy were eligible for inclusion in trial NCT01210378. In this trial, standard radiotherapy is combined with a nitroglycerin patch (Transiderm Nitro $5 \mathrm{mg}$, Novartis) on each day of irradiation. These patches are placed on hairless skin. They contain $25 \mathrm{mg} / 10 \mathrm{~cm}^{2}$ and release $0.2 \mathrm{mg}$ of nitroglycerin per hour. Patient characteristics are summarized in Supplementary Table S2. To study the mechanisms underlying a potential treatment effect, patients enrolled in this trial were also requested to undergo facultative scans to measure the effect of nitroglycerin on both tumor hypoxia and perfusion. Hypoxia was evaluated by hypoxia PET-scans using the 2nitroimidazole $\left[{ }^{18} \mathrm{~F}\right]-\mathrm{HX} 4$ (flortanidazole, 3-[18F]fluoro-2-(4-((2-nitro-1Himidazol-1yl)methyl)-1H-1,2,3-triazol-1-yl)-propan-1-ol, from here on referred to as HX4). Tumor perfusion was investigated by dynamic contrast enhanced CT-scanning (DCE-CT).

Prior to the start of radiotherapy DCE-CT and HX4 scans were made to measure baseline tumor perfusion and hypoxia, respectively. At least 48 hours later these DCE-CT and HX4 scans were repeated, the so-called nitroglycerin scans, at a minimum of 1 hour after application of a Transiderm Nitro $5 \mathrm{mg}$ patch, following the window-ofopportunity concept (Fig. 6) [12].

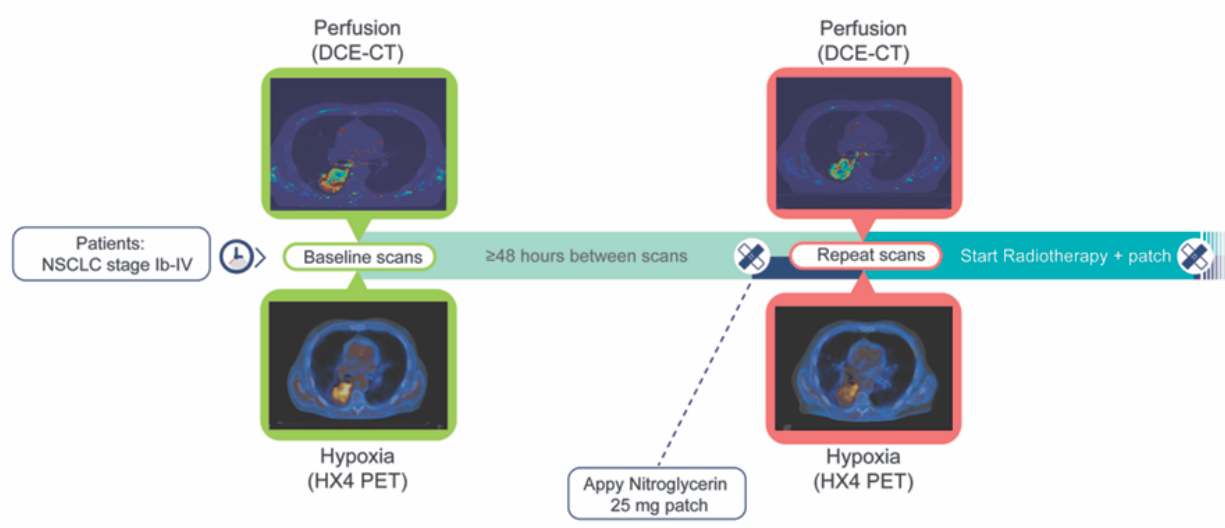

Figure 6. Flow chart of study design. 


\section{HX4 PET image acquisition}

The production of HX4 has been extensively described in previous publications [14-18, $39,40,47-49]$. Four hours after intravenous injection of $429 \pm 57 \mathrm{MBq}$ of HX4 a PET/CT scan was performed consisting of a single bed position centered on the primary tumor (Gemini TF 64 scanner, Philips Healthcare, Best, the Netherlands). Spatial resolution was +/-5 mm FWHM. CT-based attenuation correction and scatter correction (SS-SIMUL) was performed and PET images were reconstructed using 3D-ordered subset iterative time-of-flight reconstruction (BLOB-OS-TF) with 3 iterations and 33 subsets in a $144 \mathrm{x}$ 144 matrix with voxel sizes of $4 \times 4 \mathrm{~mm}$. Patients were scanned in treatment position for both imaging modalities were positioned using a laser alignment system on a flat tabletop with their arms resting in an arm rest positioned above the head.

\section{DCE-CT-image acquisition}

DCE-CT scans were acquired as previously described [15]. We used a second-generation dual source CT scanner (SOMATOM Definition Flash; Siemens Healthcare). Patients received 60 or $65 \mathrm{ml}$ of iodinated contrast medium (Iopromide 300; Bayer Healthcare, Berlin, Germany) at a flow rate of $7 \mathrm{ml} / \mathrm{s}$ (iodine delivery rate $2.1 \mathrm{~g} / \mathrm{s}$ ), followed by a saline chaser of 30 or $40 \mathrm{ml}$ at the same flow rate. The scan consisted of a first pass volumetric perfusion acquisition with a serial acquisition every $1.5 \mathrm{~s}$ at $80 \mathrm{kVp}$ of the part of the thorax centered on the primary tumor (scan length $12 \mathrm{~cm}$ ) for a period of approximately 50 seconds (33 consecutive scans). Patients were imaged in expiration breath-hold and asked to continue shallow breathing if holding their breath was no longer possible.

Reconstruction was done using a B20f filter with a slice thickness of $5 \mathrm{~mm}$ and a slice increment of $3 \mathrm{~mm}$. CT images were registered afterwards to correct for breathing motion, a noise reduction was performed and a deconvolution approach was used for the kinetic modelling of the perfusion parameters implemented in commercially available software (Siemens VPCT body; Siemens Healthcare).

Tumor delineation and image analysis

In each patient the gross tumor volume of the primary tumor (GTVp) and the involved lymph nodes (GTVIn) were delineated and checked by at least 2 radiation oncologists on a planning FDG PET/CT scan. We performed a rigid registration of the planning CT to the HX4/ CT to enable the use of the GTVs on the HX4 PET/CT and a registration of the DCE$\mathrm{CT}$ blood flow and blood volume maps to the planning CT. The registration software was developed in-house [50]. For HX4 image analysis, the mean uptake of HX4 in a volume of interest in the aorta was chosen as background. GTVp and GTVIn were analyzed separately. We determined the following values for each patient: maximum and mean standardized uptake values (SUV $\max$ and $S U V_{\text {mean }}$ ) of HX4 and the maximum tumor-to- 
blood ratio (TBR), defined as the SUV $V_{\text {max }}$ in GTVp and/or GTVIn divided by the SUV mean in the aorta.

A TBR $>1.4$ was chosen as the cut-off signal intensity defining presence of hypoxia [17, 18]. The fraction and volume of a GTV with a TBR $>1.4$ was considered as the HX4 highuptake fraction (HX4-HF) and HX4 high-uptake volume (HX4-HV), respectively. For the DCE-CT imaging the average perfusion parameters blood flow $(\mathrm{ml} / 100 \mathrm{ml} / \mathrm{min})$ and blood volume $(\mathrm{ml} / 100 \mathrm{ml})$ were calculated within GTVp.

\section{Molecular experiments}

Cell culture

Human non-small cell lung adenocarcinoma H522 cells were cultured in RPMI 1640 (Lonza). Human non-small lung carcinoma cell lines H1299 wild-type and H1299 overexpressing the bacterial nitroreductase NfsA (H1299 NfsA) (kindly provided by Adam Patterson, Auckland University, New Zealand) were cultured in high glucose (4,5g/L) DMEM (Lonza). NfsA overexpression in H1299 cells was obtained via a method as described by Prosser et al. [51]. Both media types were supplemented with $10 \%$ fetal bovine serum (Sigma-Aldrich).

Cellular respiration assays

Oxygen consumption rates (OCRs) were determined using an extracellular Flux analyzer (Seahorse XF96, Seahorse Bioscience). In a 96-well XF96 cell culture plate $3.0 \times 10^{4} \mathrm{H} 522$ and $1.5 \times 10^{4} \mathrm{H} 1299$ or $\mathrm{H} 1299 \mathrm{NfsA}$ cells/well were seeded respectively in $80 \mu$ l culture medium and placed in a $5 \% \mathrm{CO}_{2}$ incubator at $37^{\circ} \mathrm{C}$ to attach. Medium was exchanged to DMEM containing $2 \mathrm{mM}$ L-glutamine and either $10 \mathrm{mM}$ glucose or galactose medium in order to prime the cells to use oxidative phosphorylation. The microplates were placed 20 hours in a $5 \% \mathrm{CO}_{2}$ incubator at $37^{\circ} \mathrm{C}$. Culture medium was exchanged 30 minutes before the assay with $175 \mu \mathrm{l}$ assay medium containing $175 \mu \mathrm{l}$ biocarbonate-free DMEM pH 7.35 +/- 0.05 (Sigma-Aldrich) supplemented with $1.85 \mathrm{~g} / \mathrm{L} \mathrm{NaCl}$ (Sigma-Aldrich), 2 $\mathrm{mM}$ L-glutamine (Gibco) and $3 \mathrm{mg} / \mathrm{L}$ Phenol Red (Sigma-Aldrich) and containing either $10 \mathrm{mM}$ of glucose or galactose. Plates were placed in a $\mathrm{CO}_{2}$-free incubator at $37^{\circ} \mathrm{C}$. Here, prior to the first injection, baseline OCR was determined using a mixing period of 5 minutes and a measurement time of 3 minutes followed by 3 loops of mixing and measuring for 3 minutes each. Medium containing vehicle (purified water) or nitroglycerin (352 pM, 352nM and $35.2 \mu \mathrm{M}$ ) (both kindly provided by Novasep) were injected followed by several mixing and measurements cycles. 


\section{In vitro assessment of pimonidazole binding by flow cytometry}

Cells (H1299 and H1299 NfsA) were seeded in a 6-wells plate at a density of $2.5 \times 10^{4}$ cells per well. The day after, cells were incubated for 16 hours under normoxic $\left(21 \% \mathrm{O}_{2}\right.$, $\left.37^{\circ} \mathrm{C}\right)$ or hypoxic $\left(0.2 \% \mathrm{O}_{2}, 37^{\circ} \mathrm{C}\right)$ conditions. This was followed by the exposure to 35.2 $\mu \mathrm{M}$ nitroglycerin $(8 \mathrm{mg} / \mathrm{l})$ or vehicle to the cells for 1 hour under either normoxia or hypoxic conditions. Note: a normal plasma concentration achieved by a Transiderm Nitro $5 \mathrm{mg}$ patch is $0.16 \pm 0.03 \mu \mathrm{g} / \mathrm{l}$ [21]. Subsequently, cells were incubated for 2 hours under either normoxia or hypoxia with $20 \mu \mathrm{M}$ pimonidazole (Bio-connect) followed by trypsinization and fixation with (4\% PFA) for 1 hour at room temperature. Cells were washed with PBS containing 1\% BSA, permeabilized with $0.2 \%$ Triton X-100 in PBS for 30 minutes at RT. Subsequently samples were incubated with 10\% BSA in PBS for 30 minutes at room temperature after which they were incubated with a rabbit antipimonidazole antibody (Hypoxyprobe) (1:50) in PBS 1\% BSA for 2 hours at $37^{\circ} \mathrm{C}$. Samples were washed with PBS and incubated with a goat anti-rabbit Alexa 488 antibody (1:500) for 30 minutes. After 3 times washing with PBS containing 1\% BSA cells were resuspended in 1\% BSA containing PBS and FACS analyses were performed.

\section{Statistics}

The clinical data are reported as median \pm 1 standard deviation (SD). Differences between paired measurements were assessed using the Wilcoxon Signed rank test. Correlation coefficients were calculated using Spearman's correlation coefficient (SPSS, IBM, Germany). The results of the molecular experiments were presented as median \pm range of at least 3 individual experiments. Means were compared using a MannWhitney-U-test (SPSS, IBM, Germany). For both the clinical data as the molecular data $p$-value of $\leq 0.05$ was assumed to be statistically significant.

\section{Acknowledgements}

Nitroglycerin and its vehicle for laboratory use were kindly provided by Cornelius Ruloff from Novasep Synthesis. Funding: Authors acknowledge the financial support provided from ERC advanced grant (ERC-ADG-2015, n 694812 - Hypoximmuno) and by the Dutch technology Foundation STW (grant n 10696 DuCAT \& n P14-19 Radiomics STRaTegy), which is the applied science division of NWO, and the Technology Program of the Ministry of Economic Affairs. Authors also acknowledge financial support from EU 7th framework program (METOXIA - $n^{\circ}$ 2008-222741, ARTFORCE - $n^{\circ} 257144$ and ImmunoSABR - $n^{\circ}$ 733008), SME Phase 2 (EU proposal 673780 - RAIL), Kankeronderzoeksfonds Limburg from the Health Foundation Limburg, the Belgian 
cancer foundation and the Dutch Cancer Society (KWF UM 2011-5020, KWF UM 20094454, KWF MAC 2013-6425, KWF MAC 2013-6089, KWF 2015-7635).

\section{Author contributions}

$B R, E V, J W, D d R$ and $P L$ were involved in designing the clinical study. MvG, LD and AY designed the preclinical studies, while MvG performed the preclinical studies. DCE-CT scans were performed under the supervision of MD. DCE-CT-image analysis, HX4 image analyses were performed by A.E. and C.Z under the supervision of WvE. BR, MvG, AE, C.Z and LD analyzed the data. All authors contributed to the scientific discussions and interpretation of the results. BR and MvG wrote the manuscript with suggestions and contributions of all authors.

\section{Competing financial interests}

None of the authors declare any competing financial interests. 


\section{Supplementary data}

A

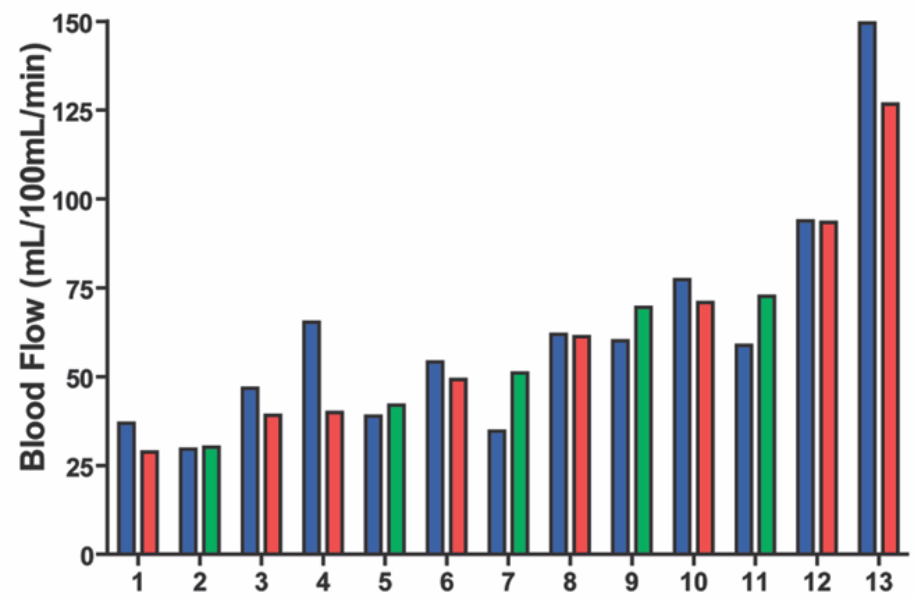

B

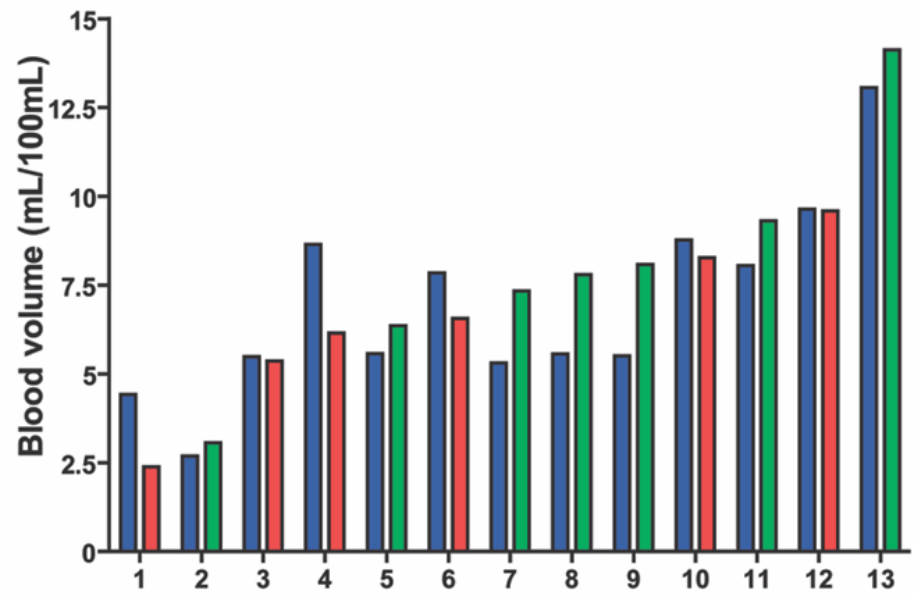

Supplementary Figure S1: Perfusion parameters in 13 patients with both a baseline DCE-CT scan and DCE-CT scan after nitroglycerin.

$A=$ tumor blood flow at baseline (blue columns) vs after nitroglycerin per patient. $B=$ tumor blood volume at baseline (blue columns) vs after nitroglycerin per patient. Green columns= increase, Red columns= decrease. 

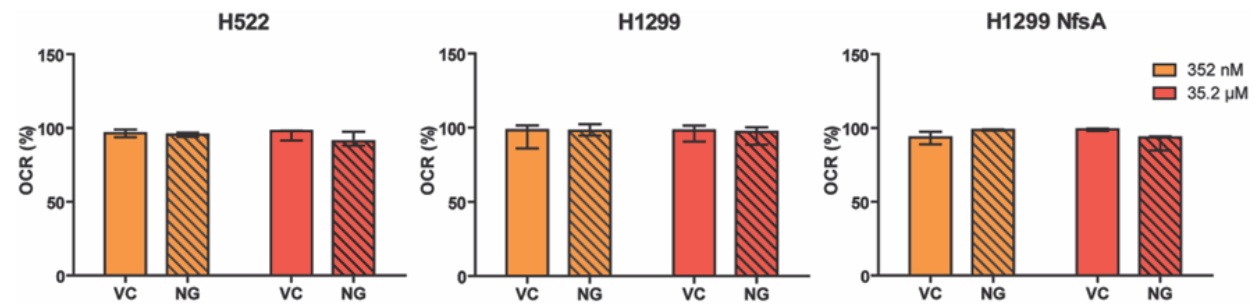

Supplementary Figure S2. Nitroglycerin at plasma concentrations does not affect cellular respiration upon galactose exposure.

Relative inhibition of mitochondrial respiration of H1299, H1299 NfsA or H522 cells upon treatment with nitroglycerin (NG, $352 \mathrm{nM}$ or $35.2 \mu \mathrm{M}$ ) compared to vehicle (VC). Measurements were normalized for protein concentration and the percentage of baseline OCR before adding nitroglycerin or vehicle. Results are shown for median \pm range of at least 3 independent experiments.

Supplementary Table S1. Overview of the performed scans and analyses

\begin{tabular}{lll}
\hline Scans & $\begin{array}{l}\text { Number of } \\
\text { patients }\end{array}$ & Analysis \\
\hline Baseline HX-4 PET & 32 & Baseline hypoxia in GTVp and GTVIn \\
Baseline HX-4 PET + Nitroglycerin HX4 PET & 25 & $\begin{array}{l}\text { Nitroglycerin effect on hypoxia in GTVp and } \\
\text { GTVln }\end{array}$ \\
Baseline DCE-CT & 22 & $\begin{array}{l}\text { Baseline perfusion in GTVp } \\
\text { Baseline DCE-CT + Baseline HX-4 PET }\end{array}$ \\
$\begin{array}{lll}\text { Correlation between baseline hypoxia and } \\
\text { Baseline DCE-CT + Nitroglycerin DCE-CT }\end{array}$ & 13 & $\begin{array}{l}\text { Nitroglycerin effect on perfusion in GTVp } \\
\text { Baseline DCE-CT + Baseline HX-4 PET + }\end{array}$ \\
Nitroglycerin DCE-CT + Nitroglycerin HX4 PET & 12 & $\begin{array}{l}\text { Correlation between nitroglycerin effect on } \\
\text { perfusion and effect on hypoxia in GTVp }\end{array}$ \\
\hline
\end{tabular}


Chapter 6

Supplementary Table S2. Patient characteristics

\begin{tabular}{llll}
\hline Parameters & & Cases $(n)$ & Percentage (\%) \\
\hline Number of patients & $62 \pm 11(40-82)$ & 34 & \\
Age years, median \pm SD (range) & & & \\
Gender & Male & 21 & 62 \\
& Female & 13 & 38
\end{tabular}

WHO-PS

$\begin{array}{lll}0 & 6 & 18 \\ 1 & 28 & 82\end{array}$

Smoking status

$\begin{array}{lll}\text { Active } & 6 & 18 \\ \text { Quit } & 26 & 76 \\ \text { Never } & 1 & 3 \\ \text { Unknown } & 1 & 3\end{array}$

Stage

$\begin{array}{lll}\text { I } & 2 & 6 \\ \text { II II } & 2 & 6 \\ \text { IV } & 19 & 56 \\ & 11 & 32\end{array}$

T-stage

$\begin{array}{lll}x & 1 & 3 \\ 1 & 0 & 0 \\ 2 & 10 & 29 \\ 3 & 10 & 29 \\ 4 & 13 & 38\end{array}$

$\mathrm{N}$-stage

$\begin{array}{lll}0 & 7 & 21 \\ 1 & 1 & 3 \\ 2 & 13 & 38 \\ 3 & 13 & 38\end{array}$

M-stage

$\begin{array}{lll}\text { M0 } & 23 & 67 \\ \text { M1a } & 4 & 12 \\ \text { M1b } & 7 & 21\end{array}$




\section{References}

1. Oronsky, B.T., S.J. Knox, and J.J. Scicinski, Is Nitric Oxide (NO) the Last Word in Radiosensitization? A Review. Transl Oncol, 2012. 5(2): p. 66-71.

2. Riganti, C., et al., Nitric oxide reverts the resistance to doxorubicin in human colon cancer cells by inhibiting the drug efflux. Cancer Res, 2005. 65(2): p. 516-25.

3. Siemens, D.R., et al., Phase /l study of nitric oxide donor for men with increasing prostate-specific antigen level after surgery or radiotherapy for prostate cancer. Urology, 2009. 74(4): p. 878-83.

4. Yasuda, H., Solid tumor physiology and hypoxia-induced chemo/radio-resistance: novel strategy for cancer therapy: nitric oxide donor as a therapeutic enhancer. Nitric Oxide, 2008. 19(2): p. 205-16.

5. Yasuda, $\mathrm{H}_{\text {., }}$ et al., Randomized phase II trial comparing nitroglycerin plus vinorelbine and cisplatin with vinorelbine and cisplatin alone in previously untreated stage IIIB/IV non-small-cell lung cancer. J Clin Oncol, 2006. 24(4): p. 688-94.

6. Bonaventura, J., Clinical implications of the loss of vasoactive nitric oxide during red blood cell storage. Proc Natl Acad Sci U S A, 2007. 104(49): p. 19165-6.

7. Brown, G.C., Nitric oxide and mitochondrial respiration. Biochim Biophys Acta, 1999. 1411(2-3): p. 351-69.

8. Hagen, T., et al., Redistribution of intracellular oxygen in hypoxia by nitric oxide: effect on HIF1alpha. Science, 2003. 302(5652): p. 1975-8.

9. Jordan, B.F., et al., Changes in tumor oxygenation/perfusion induced by the no donor, isosorbide dinitrate, in comparison with carbogen: monitoring by EPR and MRI. Int J Radiat Oncol Biol Phys, 2000. 48(2): p. 565-70.

10. Zlotecki, R.A., et al., Pharmacologic modification of tumor blood flow and interstitial fluid pressure in a human tumor xenograft: network analysis and mechanistic interpretation. Microvasc Res, 1995. 50(3): p. 429-43.

11. Lambin, P., et al., Decision support systems for personalized and participative radiation oncology. Adv Drug Deliv Rev, 2016.

12. Dubois, L.J., et al., New ways to image and target tumour hypoxia and its molecular responses. Radiotherapy and oncology : journal of the European Society for Therapeutic Radiology and Oncology, 2015. 116(3): p. 352-7.

13. Glimelius, B. and M. Lahn, Window-of-opportunity trials to evaluate clinical activity of new molecular entities in oncology. Annals of oncology : official journal of the European Society for Medical Oncology / ESMO, 2011. 22(8): p. 1717-25.

14. Zegers, C.M., et al., Evaluation of tumour hypoxia during radiotherapy using [F]HX4 PET imaging and blood biomarkers in patients with head and neck cancer. European journal of nuclear medicine and molecular imaging, 2016.

15. van Elmpt, W., et al., Multiparametric imaging of patient and tumour heterogeneity in non-small-cell lung cancer: quantification of tumour hypoxia, metabolism and perfusion. Eur J Nucl Med Mol Imaging, 2016. 43(2): p. 240-8.

16. Zegers, C.M., et al., Imaging of tumour hypoxia and metabolism in patients with head and neck squamous cell carcinoma. Acta oncologica, 2015. 54(9): p. 1378-84.

17. Zegers, C.M., et al., Repeatability of hypoxia PET imaging using [(1)(8)F]HX4 in lung and head and neck cancer patients: a prospective multicenter trial. Eur J Nucl Med Mol Imaging, 2015. 42(12): p. 1840-9.

18. Zegers, C.M., et al., In vivo quantification of hypoxic and metabolic status of NSCLC tumors using [18F]HX4 and [18F]FDG-PET/CT imaging. Clinical cancer research : an official journal of the American Association for Cancer Research, 2014. 20(24): p. 6389-97.

19. Lambin, P., et al., Predicting outcomes in radiation oncology--multifactorial decision support systems. Nature reviews. Clinical oncology, 2013. 10(1): p. 27-40.

20. Reymen, B., et al., Data from: Nitroglycerin in non-small cell lung cancer: does it impact tumor hypoxia and tumor perfusion? A window-of-opportunity clinical trial., 2016, Cancer Data. 
21. McAllister, A., et al., Plasma levels of nitroglycerin generated by three nitroglycerin patch preparations, Nitradisc, Transiderm-Nitro and Nitro-Dur and one ointment formulation, Nitrobid. Br J Clin Pharmacol, 1986. 21(4): p. 365-9.

22. Chang, C.F., A.R. Diers, and N. Hogg, Cancer cell metabolism and the modulating effects of nitric oxide. Free radical biology \& medicine, 2015. 79: p. 324-36.

23. Siemens, D.R., et al., Hypoxia increases tumor cell shedding of MHC class I chain-related molecule: role of nitric oxide. Cancer Res, 2008. 68(12): p. 4746-53.

24. Sonveaux, P., et al., Nitric oxide delivery to cancer: why and how? Eur J Cancer, 2009. 45(8): p. 1352-69.

25. Reinmuth, N., et al., Randomized, double-blind phase /I study to compare nitroglycerin plus oral vinorelbine plus cisplatin with oral vinorelbine plus cisplatin alone in patients with stage IIIB/IV non-small cell lung cancer (NSCLC). Lung Cancer, 2014. 83(3): p. 363-8.

26. Han, J.Y., et al., A randomized phase II study of irinotecan plus cisplatin versus irinotecan plus capecitabine with or without isosorbide-5-mononitrate in advanced non-small-cell lung cancer. Ann Oncol, 2012. 23(11): p. 2925-30.

27. Dingemans, A.M., et al., A randomized phase I/ study comparing paclitaxel-carboplatin-bevacizumab with or without nitroglycerin patches in patients with stage IV nonsquamous nonsmall-cell lung cancer: NVALT12 (NCT01171170)dagger. Ann Oncol, 2015. 26(11): p. 2286-93.

28. Davidson, A., et al., A phase III randomized trial of adding topical nitroglycerin to first-line chemotherapy for advanced nonsmall-cell lung cancer: the Australasian lung cancer trials group NITRO trial. Annals of oncology : official journal of the European Society for Medical Oncology / ESMO, 2015. 26(11): p. 2280-6.

29. Arrieta, O., et al., Phase I/ study. Concurrent chemotherapy and radiotherapy with nitroglycerin in locally advanced non-small cell lung cancer. Radiother Oncol, 2014. 111(2): p. 311-5.

30. Yasuda, H., et al., Nitroglycerin treatment may enhance chemosensitivity to docetaxel and carboplatin in patients with lung adenocarcinoma. Clin Cancer Res, 2006. 12(22): p. 6748-57.

31. Dungel, P., et al., Neither nitrite nor nitric oxide mediate toxic effects of nitroglycerin on mitochondria. J Biochem Mol Toxicol, 2011. 25(5): p. 297-302.

32. Brown, G.C., Nitric oxide as a competitive inhibitor of oxygen consumption in the mitochondrial respiratory chain. Acta Physiol Scand, 2000. 168(4): p. 667-74.

33. Brown, G.C. and C.E. Cooper, Nanomolar concentrations of nitric oxide reversibly inhibit synaptosomal respiration by competing with oxygen at cytochrome oxidase. FEBS Lett, 1994. 356(2-3): p. 295-8.

34. Caneba, C.A., et al., Nitric oxide is a positive regulator of the Warburg effect in ovarian cancer cells. Cell death \& disease, 2014. 5: p. e1302.

35. Gohil, V.M., et al., Nutrient-sensitized screening for drugs that shift energy metabolism from mitochondrial respiration to glycolysis. Nat Biotechnol, 2010. 28(3): p. 249-55.

36. Bonora, E., et al., Defective oxidative phosphorylation in thyroid oncocytic carcinoma is associated with pathogenic mitochondrial DNA mutations affecting complexes I and III. Cancer Res, 2006. 66(12): p. 6087-96.

37. Mocellin, S., V. Bronte, and D. Nitti, Nitric oxide, a double edged sword in cancer biology: searching for therapeutic opportunities. Med Res Rev, 2007. 27(3): p. 317-52.

38. Shan, S.Q., et al., Effects of diethylamine/nitric oxide on blood perfusion and oxygenation in the R3230AC mammary carcinoma. Br J Cancer, 1997. 76(4): p. 429-37.

39. Peeters, S.G., et al., A comparative study of the hypoxia PET tracers [(1)(8)F]HX4, [(1)(8)F]FAZA, and [(1)(8)F]FMISO in a preclinical tumor model. Int J Radiat Oncol Biol Phys, 2015. 91(2): p. 351-9.

40. Dubois, L.J., et al., Preclinical evaluation and validation of [18F]HX4, a promising hypoxia marker for PET imaging. Proceedings of the National Academy of Sciences of the United States of America, 2011. 108(35): p. 14620-5.

41. Zegers, C.M., et al., Hypoxia imaging with [(1)(8)F]HX4 PET in NSCLC patients: defining optimal imaging parameters. Radiotherapy and oncology : journal of the European Society for Therapeutic Radiology and Oncology, 2013. 109(1): p. 58-64.

42. Ng, Q.S., et al., Lung cancer perfusion at multi-detector row CT: reproducibility of whole tumor quantitative measurements. Radiology, 2006. 239(2): p. 547-53. 
43. Sauter, A.W., et al., Intraobserver and interobserver agreement of volume perfusion CT (VPCT) measurements in patients with lung lesions. European journal of radiology, 2012. 81(10): p. 2853-9.

44. Garcia-Bou, R., et al., Evidence for a relationship between mitochondrial Complex I activity and mitochondrial aldehyde dehydrogenase during nitroglycerin tolerance: effects of mitochondrial antioxidants. Biochimica et biophysica acta, 2012. 1817(5): p. 828-37.

45. Aerts, H.J., N.A. van Riel, and W.H. Backes, System identification theory in pharmacokinetic modeling of dynamic contrast-enhanced MRI: influence of contrast injection. Magn Reson Med, 2008. 59(5): p. 1111-9.

46. Lambin, P., et al., Radiomics: extracting more information from medical images using advanced feature analysis. Eur J Cancer, 2012. 48(4): p. 441-6.

47. Chen, L., et al., (1)(8)F-HX4 hypoxia imaging with PET/CT in head and neck cancer: a comparison with (1)(8)F-FMISO. Nuclear medicine communications, 2012. 33(10): p. 1096-102.

48. Doss, M., et al., Biodistribution and radiation dosimetry of the hypoxia marker 18F-HX4 in monkeys and humans determined by using whole-body PET/CT. Nuclear medicine communications, 2010. 31(12): p. 1016-24.

49. van Loon, J., et al., PET imaging of hypoxia using [18F]HX4: a phase I trial. European journal of nuclear medicine and molecular imaging, 2010. 37(9): p. 1663-8.

50. Janssens, G., et al., Diffeomorphic registration of images with variable contrast enhancement. International journal of biomedical imaging, 2011. 2011: p. 891585.

51. Prosser, G.A., et al., Creation and screening of a multi-family bacterial oxidoreductase library to discover novel nitroreductases that efficiently activate the bioreductive prodrugs CB1954 and PR-104A. Biochemical pharmacology, 2013. 85(8): p. 1091-103. 



\section{Chapter}

\section{Chemical reactivity window determines prodrug efficiency toward glutathione transferase overexpressing cancer cells}

Reprinted with permission from Molecular pharmaceutics, 2016. 13(6): p. 2010-25. Copyright (2016) American Chemical Society.

doi: 10.1021/acs.molpharmaceut.6b00140

Marike W. van Gisbergen*, Marcus Cebula*, Jie Zhang, Astrid Ottosson-Wadlund, Ludwig Dubois, Philippe Lambin, Kenneth D. Tew, Danyelle M. Townsend, Guido R. M. M. Haenen, Marie-José Drittij-Reijnders, Hisao Saneyoshi, Mika Araki, Yuko Shishido, Yoshihiro Ito, Elias S. J. Arnér, Hiroshi Abe, Ralf Morgenstern, Katarina Johansson

* contributed equally 


\section{Abstract}

Glutathione transferases (GSTs) are often overexpressed in tumors and frequently correlated to bad prognosis and resistance against a number of different anticancer drugs. To selectively target these cells and to overcome this resistance we previously have developed prodrugs that are derivatives of existing anticancer drugs (e.g., doxorubicin) incorporating a sulfonamide moiety. When cleaved by GSTs, the prodrug releases the cytostatic moiety predominantly in GST overexpressing cells, thus sparing normal cells with moderate enzyme levels. By modifying the sulfonamide it is possible to control the rate of drug release and specifically target different GSTs. Here we show that the newly synthesized compounds, 4-acetyl-2-nitro-benzenesulfonyl etoposide (ANS-etoposide) and 4-acetyl-2-nitro-benzenesulfonyl doxorubicin (ANS-DOX), function as prodrugs for GSTA1 and MGST1 overexpressing cell lines. ANS-DOX, in particular, showed a desirable cytotoxic profile by inducing toxicity and DNA damage in a GST-dependent manner compared to control cells. Its moderate conversion of $500 \mathrm{nmol} / \mathrm{min} / \mathrm{mg}$, as catalyzed by GSTA1, seems hereby essential since the more reactive 2,4-dinitrobenzenesulfonyl doxorubicin (DNS-DOX) (14000 nmol/min/mg) did not display a preference for GSTA1 overexpressing cells. DNS-DOX, however, effectively killed GSTP1 $(20 \mathrm{nmol} / \mathrm{min} / \mathrm{mg})$ and MGST1 (450 nmol/min/mg) overexpressing cells as did the less reactive 4mononitrobenzenesulfonyl doxorubicin (MNS-DOX) in a MGST1-dependent manner (1.5 $\mathrm{nmol} / \mathrm{min} / \mathrm{mg}$ ) as shown previously. Furthermore, we show that the mechanism of these prodrugs involves a reduction in GSH levels as well as inhibition of the redox regulatory enzyme thioredoxin reductase 1 (TrxR1) by virtue of their electrophilic sulfonamide moiety. TrxR1 is upregulated in many tumors and associated with resistance to chemotherapy and poor patient prognosis. Additionally, the prodrugs potentially acted as a general shuttle system for DOX, by overcoming resistance mechanisms in cells. Here we propose that GST-dependent prodrugs require a conversion rate "window" in order to selectively target GST overexpressing cells, while limiting their effects on normal cells. Prodrugs are furthermore a suitable system to specifically target GSTs and to overcome various drug resistance mechanisms that apply to the parental drug.

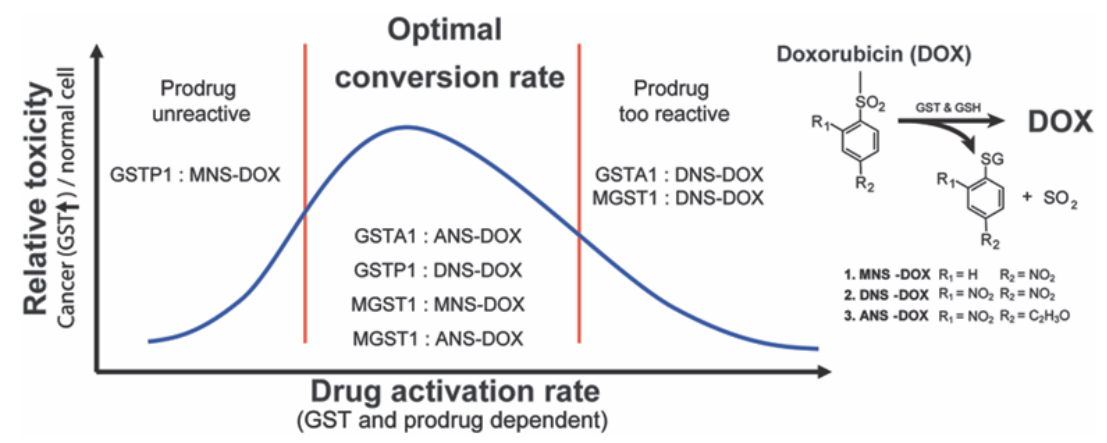




\section{Introduction}

Cancer is one of the leading causes of death in privileged countries. In patients suffering from cancer, the choice of therapy depends on several factors, such as the histopathology of the tumor, the stage of the disease, and the patient's condition. Chemotherapy is commonly used as anticancer treatment, but patients often suffer from severe side effects, which are of major concern. Especially highly proliferating cells, present in the bone marrow, hair follicles, and gastrointestinal mucosa, are affected by conventional chemotherapeutics leading to common side effects like myelosuppression, alopecia, and mucositis [1]. Further, there are additional and more specific side effects depending on the cytostatic drug used. Doxorubicin (DOX), e.g., a common topoisomerase II inhibitor that is used to treat patients with different solid and hematological malignancies, can also cause cardiotoxicity [2] and eventually congestive heart failure [3]. Another major problem that can limit the effectiveness of cancer treatment is drug resistance. Drug resistance is related to numerous changes in the tumor and its microenvironment [4] and can reflect both tumor and normal tissue [5]. An approach to reduce side effects and drug resistance is the usage of prodrugs. Prodrugs are chemically modified, preferably nontoxic, versions of the active anticancer agent. Upon entrance into cancer cells the prodrug can be activated whereupon the active cytostatic molecule is released. Activation is typically catalyzed by an enzyme that is highly abundant in cancer cells, leading to predominant high concentrations in tumor cells while sparing normal cells [6].A suggested enzyme family to be targeted to activate prodrugs in cancer cells is glutathione transferases (GSTs) [7-15]. The GSTs are often highly overexpressed in tumors and frequently correlated to drug resistance and bad prognosis $[16,17]$. GSTs are divided into three different groups, cytosolic, mitochondrial, and microsomal GSTs, each containing various isoforms. These isoforms have specific as well as overlapping substrate specificities in catalyzing conjugation, reduction, and isomerization reactions, thereby contributing to cellular protection against chemical insult, oxidative stress, and lipid peroxidation. Furthermore, GSTs are suggested to have essential functions in cell signaling $[12,15,18-20]$.

In an attempt to take advantage of the overexpression of GSTs in different tumors we previously presented a new strategy using two different forms of prodrugs that can be activated and thereby release DOX in GST overexpressing cells. We showed that these prodrugs, 4-mononitrobenzenesulfonyl doxorubicin (MNS-DOX) and 2,4-dinitrobenzenesulfonyl doxorubicin (DNS-DOX), could be activated by two different GSTs, microsomal glutathione transferase 1 (MGST1) and glutathione transferase pi (GSTP). MGST1, which is located in the ER membrane, and the outer membrane of mitochondria as well as the cytosolic GSTP are often overexpressed, connected to drug resistance and correlated to bad prognosis in many different cancers. Our study showed that the prodrugs tested were 
more toxic to GST overexpressing cells compared to control cells, indicating that the GSTs tested could activate the prodrugs and release DOX [8].

A<smiles>COc1cccc2c1C(=O)c1c(O)c3c(c(O)c1C2=O)[C@H](O[C@H]1C[C@H](N)[C@H](O)[C@H](C)O1)C[C@@](O)(C(=O)CO)C3</smiles>

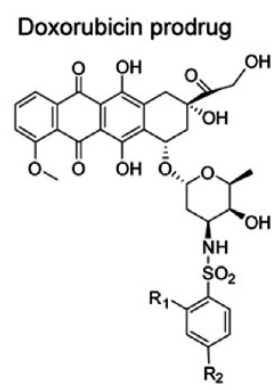

1. MNS-DOX $\mathrm{R}_{1}=\mathrm{H} \quad \mathrm{R}_{2}=\mathrm{NO}_{2}$

2. DNS-DOX $R_{1}=\mathrm{NO}_{2} \quad \mathrm{R}_{2}=\mathrm{NO}_{2}$

3. ANS-DOX $R_{1}=\mathrm{NO}_{2} \quad \mathrm{R}_{2}=\mathrm{C}_{2} \mathrm{H}_{3} \mathrm{O}$

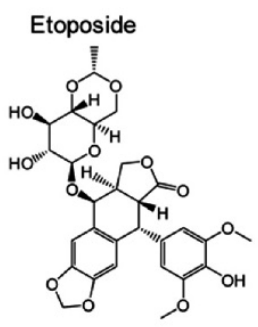

Etoposide produg

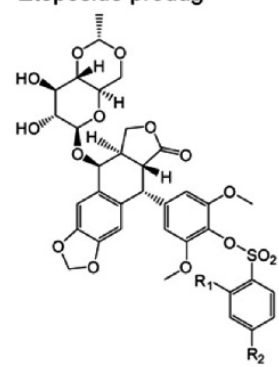

1. ANS-Etoposide $\mathrm{R}_{1}=\mathrm{NO}_{2} \quad \mathrm{R}_{2}=\mathrm{C}_{2} \mathrm{H}_{3} \mathrm{O}$

B

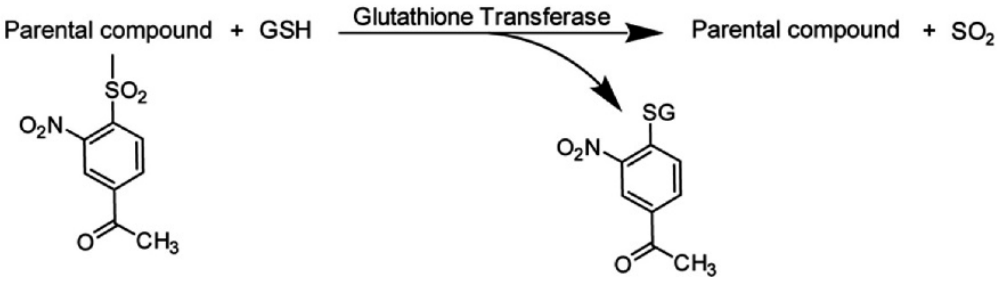

Figure 1. Chemical structures and conversion of prodrugs.

A. Chemical structures of DOX and etoposide as well as the prodrugs MNS-DOX, DNS-DOX, ANS-DOX, and ANS-etoposide are depicted. B. Conversion of the prodrug yields a free doxorubicin/etoposide, sulfur dioxide and an acetylmononitro/dinitro benzene moiety conjugated to GSH. These prodrugs are hypothesized to enter the cell via a passive diffusion. In the cell, GSTs are able to activate the prodrugs via their sulfonamidase and sulfonamide cleavage activity.

To follow up on these findings we herein expanded our efforts to glutathione transferase alpha 1 (GSTA1) and included the newly synthesized DOX derivative 4acetyl-2-nitro DOX (ANS-DOX) in the study (Fig. 1). GSTA1 is expressed in most cells at moderate levels and has been shown to possess both GST and glutathione peroxidase activity protecting against oxidative stress [15]. However, GSTA1 has also been shown to be overexpressed in various cancers such as colon cancer [21], lung cancer [22, 23], exfoliated human bladder cancer[24], and clear cell renal cell carcinoma [25] and is connected to nitrogen mustard and Bleomycin resistance [26, 27]. Overexpression was furthermore found to support proliferation of cells in benign adrenocortical adenomas from patients with Cushing's syndrome [28]. In contrast to MGST1 and GSTP1, GSTA1 overexpression was not associated with a major resistance to doxorubicin [29, 30], thus potentially being a more effective enzyme to target for these prodrugs. 

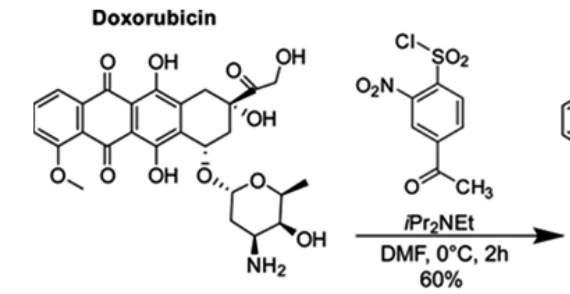

ANS-DOX(1)
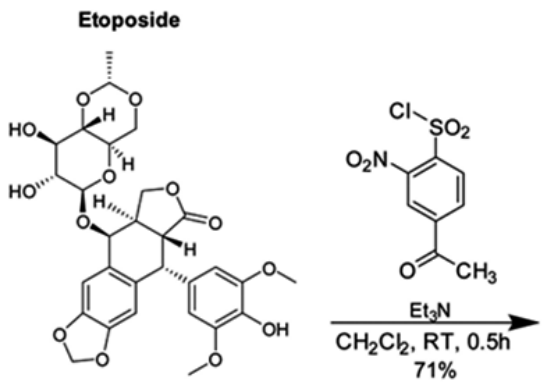

ANS-Etoposide(2)
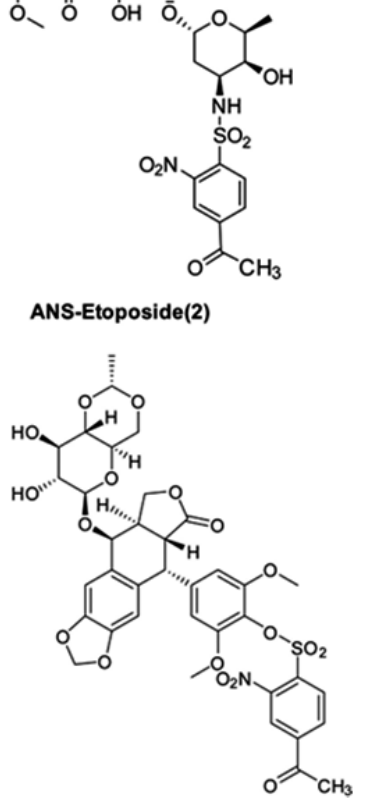

Scheme 1. Synthesis of ANS-DOX (1) and ANS-Etoposide (2); See Materials and Methods for Details

Furthermore, the prodrug concept may include additional functional facets contributing to its cytotoxic profile, which we wished to address within this study. An efficient accumulation and activation within the cancer cell, for example, might lead to GSH depletion, which in turn sensitizes the cells to the activated drug or induces oxidative stress as a concomitant factor. Additionally, intracellular nucleophiles other than GSH might be efficiently targeted due to the electrophilic nature of the sulfonamide bond in the prodrugs. A prime candidate is the selenoprotein thioredoxin reductase 1 (TrxR1), which is the central enzyme of the redox regulatory and antioxidant thioredoxin (Trx) system. It is often upregulated in cancers and has been identified as a promising target for anticancer therapy [31]. Due to an accessible and highly reactive Sec-residue within its active site, it is considered to be a target of many electrophilic compounds with TrxR1 inhibition being linked to cancer cell death [32]. Reducing the antioxidant capacity of cancer cells through targeting of the Trx and GSH systems simultaneously has previously been suggested as a principle of anticancer therapy and might thus be synergistic factors contributing to prodrug induced toxicity [33-35].

In addition, we investigated whether etoposide could be converted into a novel prodrug using a similar strategy as for DOX. The parental compound etoposide is used as a cytostatic drug for the treatment of Ewing's sarcomas, lung cancer, lymphomas, and, in 
addition, in combination with carboplatin to treat primary brain tumors such as glioblastomas [36-40]. Etoposide can act against topoisomerase II and thereby influence the chromosome segregation and replication in the exposed cancer cell resulting in apoptosis [40, 41] A major problem with etoposide is that it can induce major side effects such as bone marrow suppression, fatigue, skin rash, diarrhea, severe delayed toxicity, and even leukemia. It is therefore an attractive candidate for the development of prodrugs to potentially reduce these side effects $[42,43]$.

In this study we show that GSTs are a suitable enzyme class for the development of novel prodrugs and that different GSTs can be targeted selectively. Specifically, we synthesized and characterized a new derivative of DOX, ANS-DOX as substrate for various GST, and studied its potential as prodrug in both GSTA1 and MGST1 overexpressing cells. Additionally, we show that the working mechanism of prodrugs involves a reduction of the GSH levels as a consequence of activation, as well as disruption of redox regulatory pathways via TrxR1 inhibition, which might synergize with the mechanism of the activated drug and augment toxicity toward cancer cells. We also ascertained whether etoposide is a suitable candidate for the development of novel prodrugs by characterizing the etoposide based prodrug, 4-acetyl-2-nitro-benzenesulfonyl etoposide (ANS-etoposide), in the same cellular systems. In summary, our data show that the GST catalyzed conversion rate can be precisely manipulated by adjusting the reactivity of the prodrugs; this is also shown to be important for both GST specificity and cell killing potential. Additionally, we suggest that prodrugs may overcome multiple drug resistance mechanisms and that they can target redox pathways, particularly those regulated via TrxR1, thus potentially targeting cancer cells that are resistant to the parental drug.

\section{Materials and Methods}

\section{Chemicals and Growth Media}

Cell culture media and additives, including Dulbecco's modified Eagle's medium (DMEM), Fetal Bovine Serum (FBS), Geneticin (G418), Phosphate-Buffered Saline (PBS), and penicilin-streptomycin were obtained from GIBCO. Hygromycin B was obtained from Invitrogen. 1-Chloro-2,4-dinitrobenzene (CDNB), reduced glutathione, and doxorubicin were purchased from Sigma-Aldrich, whereas etoposide was purchased from Vepesid, Bristol-Myers. Coommassie Plus protein assay was purchased from Thermo Scientific. Horseradish peroxidase labeled goat antirabbit IgG was obtained from DakoCytomation. Other reagents for SDS-polyacrylamide gel electrophoresis and Western blot analysis were obtained from Sigma-Aldrich. Development of the blots was done by the usages of Amersham EL Western blotting reagents obtained from GE healthcare. Additionally, 3- 
(4,5-dimethylthiazol-2-yl)-2,5-diphenyltetrazolium bromide (MTT) and crystal violet were purchased from Sigma-Aldrich.

\section{Synthesis of the prodrugs}

Prodrugs were synthesized as depicted in Scheme 1 for ANS-DOX (upper panel) and ANS-etoposide (lower panel). DNS-DOX was synthesized as previously described by Johansson et al.[8] Emission spectra were obtained using an EnSpire 2300 Multilabel Reader (PerkinElmer). All spectra were measured in a 96-well optical bottom plate (Fisher Scientific, \#265301) with $10 \mu \mathrm{M}$ DOX, ANS-DOX, and DNS-DOX in a $200 \mu \mathrm{L}$ volume. Measurements were taken at room temperature in $0.1 \mathrm{M}$ potassium phosphate buffer pH 6.5. Excitation wavelength was $480 \mathrm{~nm}$, and emission was recorded between 500 and $700 \mathrm{~nm}$.

\section{Synthesis of ANS-DOX (1)}

$\mathrm{N}, \mathrm{N}$-Diisopropylethylamine $(60 \mu \mathrm{L}, 340 \mu \mathrm{mol})$ was added to a solution of doxorubicin hydrochloride $(15 \mathrm{mg}, 26 \mu \mathrm{mol})$ in dimethylformamide $(520 \mu \mathrm{L})$ at $0{ }^{\circ} \mathrm{C}$. 4-Acetyl-2nitrobenzenesulfonyl chloride $(15 \mathrm{mg}, 58 \mu \mathrm{mol})$ was added to the reaction mixture. After stirring for $2 \mathrm{~h}$, the reaction mixture was diluted with $\mathrm{CHCl}_{3}$ and washed with saturated $\mathrm{NaHCO}_{3}(\mathrm{aq})$. The organic layer was dried over $\mathrm{Na}_{2} \mathrm{SO}_{4}$ and evaporated in vacuo. The residue was purified by a neutral flash silica gel column chromatography and eluted with $\mathrm{CHCl}_{3}$-acetone $(3: 1, \mathrm{v} / \mathrm{v}$ ) to give the desired ANS-DOX (12 mg, $16 \mu \mathrm{mol}$, 60\%). ${ }^{1} \mathrm{H}$ NMR (500 MHz, DMSO-d $\left.\mathrm{d}_{6}\right): \delta 8.26(1 \mathrm{H}, \mathrm{s}, \mathrm{AcNs}), 8.16(1 \mathrm{H}, \mathrm{d}, \mathrm{dox}, J=1.8 \mathrm{~Hz})$, $8.14(1 \mathrm{H}, \mathrm{d}, \mathrm{dox}, J=1.8 \mathrm{~Hz}), 8.11-8.10(1 \mathrm{H}, \mathrm{d}, \mathrm{AcNs}, J=8.0 \mathrm{~Hz}), 8.10-8.08(1 \mathrm{H}, \mathrm{d}, \mathrm{AcNs}, J$ $=8.0 \mathrm{~Hz}), 7.86-7.80(2 \mathrm{H}, \mathrm{m}, \mathrm{dox}), 7.59-7.58(1 \mathrm{H} . \mathrm{d}, \mathrm{dox}, J=8.6 \mathrm{~Hz}), 5.38(1 \mathrm{H}, \mathrm{s}, \mathrm{dox})$, $5.15(1 \mathrm{H}, \mathrm{s}, \mathrm{dox}), 4.82-4.83(1 \mathrm{H}, \mathrm{t}, \mathrm{dox}), 4.55(2 \mathrm{H}, \mathrm{s}, \mathrm{dox}), 4.11-4.07(1 \mathrm{H}, \mathrm{d}-\mathrm{d}, \mathrm{dox}, \mathrm{J}=$ 6.9, $6.3 \mathrm{~Hz}), 3.96(3 \mathrm{H}, \mathrm{s}, \mathrm{dox}), 3.62-3.57(1 \mathrm{H}, \mathrm{d}-\mathrm{q}, \mathrm{dox}, J=4.5,4.5,5.1,4.0 \mathrm{~Hz}), 3.50(1 \mathrm{H}$, s, dox), 2.90-2.89 (2H, d, dox, J = 6.3 Hz), $2.47(1 \mathrm{H}, \mathrm{s}, \mathrm{dox}), 2.12-2.07(2 \mathrm{H}, \mathrm{m}, \mathrm{dox})$, 1.93-1.87 (1H, s-t, dox, $J=4.6,3.4,4.6 \mathrm{~Hz}), 1.40-1.37(1 \mathrm{H}, \mathrm{d}-\mathrm{d}, J=5.1,5.1 \mathrm{~Hz}), 1.21$ $(3 \mathrm{H}, \mathrm{s}, \mathrm{AcNs}), 1.09-1.08(3 \mathrm{H}, \mathrm{d}, \mathrm{dox}, J=5.7 \mathrm{~Hz}), 1.17-1.16(1 \mathrm{H}, \mathrm{t}, \mathrm{dox}) .{ }^{13} \mathrm{C}$ NMR $(125$ $\left.\mathrm{MHz}, \mathrm{DMSO}-\mathrm{d}_{6}\right): \delta 214.21,195.984,186.90,186.85,161.25,156.56,154.86,147.94$, $140.73,137.79,135.84,135.07,134.56,131.96,130.54,124.26,120.40,119.45$, 111.15, 111.09, 100.33, 79.71, 75.41, 70.47, 69.39, 67.19, 64.16, 57.07, 56.36, 50.82, $32.66,32.61,30.13,27.37,17.46$. LRMS (ESI) m/z: $\left[\mathrm{M}-\mathrm{H}^{-}\right]$calcd for $\mathrm{C}_{35} \mathrm{H}_{34} \mathrm{~N}_{2} \mathrm{O}_{16} \mathrm{~S}$, 770.16; found, 769.04 .

\section{Synthesis of ANS-Etoposide (2)}

Etoposide (10 mg, $17 \mu \mathrm{mol}$ ) was dissolved in $\mathrm{CH}_{2} \mathrm{Cl}_{2}(200 \mu \mathrm{L})$. To the solution was added $\mathrm{Et}_{3} \mathrm{~N}(3 \mu \mathrm{L}, 34 \mu \mathrm{mol})$ and 4-acetyl-2-nitrobenzenesulfonyl chloride (5 mg, $\left.19 \mu \mathrm{mol}\right)$, and the mixture was stirred at room temperature for $30 \mathrm{~min}$. The mixture was purified by preparative layer chromatography eluted with EtOAc/toluene $(3: 1, v / v)$ to give the 
desired ANS-etoposide (10 mg, $12 \mu \mathrm{mol}, 71 \%) .{ }^{1} \mathrm{H}$ NMR $\left(300 \mathrm{MHz}, \mathrm{CDCl}_{3}\right): \delta 8.38(1 \mathrm{H}$, s), $8.22(1 \mathrm{H}, \mathrm{s}), 6.85(1 \mathrm{H}, \mathrm{s}), 6.53(1 \mathrm{H}, \mathrm{s}), 6.24(2 \mathrm{H}, \mathrm{s}), 6.03-6.00(2 \mathrm{H}, \mathrm{dd}, J=0.98,7.81)$, $4.93(1 \mathrm{H}, \mathrm{d}, J=3.42), 4.77(1 \mathrm{H}, \mathrm{q}, J=4.88), 4.66(2 \mathrm{H}, \mathrm{dd}, J=7.32,11.23), 4.44(1 \mathrm{H}, \mathrm{t}, J=$ 9.23), $4.29(1 \mathrm{H}, \mathrm{t}, J=8.30), 4.21(1 \mathrm{H}, \mathrm{dd}, J=3.91,11.23), 3.72-3.27(12 \mathrm{H}, \mathrm{m}), 2.85-2.82$ $(6 \mathrm{H}, \mathrm{m}), 1.41(3 \mathrm{H}, \mathrm{d}, J=5.37) .{ }^{13} \mathrm{C} \operatorname{NMR}\left(75 \mathrm{MHz}, \mathrm{CDCl}_{3}\right) ; \delta 194.5,174.7,152.2,148.9$, $148.4,147.5,141.2,139.8,135.1,131.7,130.9,128.5,127.2,124.1,123.9,110.6,109.1$, 107.6, 101.8, 79.7, 74.5, 73.1, 68.0, 66.4, 56.0, 41.1, 37.5, 26.9, 20.2. LRMS (ESI) m/z: $\left[\mathrm{M}+\mathrm{Na}^{+}\right]$calcd for $\mathrm{C}_{37} \mathrm{H}_{37} \mathrm{NNaO}_{18} \mathrm{~S}^{+}, 838.16$; found, 838.47.

\section{Activation of prodrugs}

The conversion of DNS-DOX and ANS-DOX to DOX was measured using a Shimadzu RF 510L fluorescent spectrophotometer from Analytical Instruments Division, Kyoto Japan, using an excitation at $480 \mathrm{~nm}$ and emission at $550 \mathrm{~nm}$. The enzymatic activation of ANS-DOX $(10 \mu \mathrm{M})$ or DNS-DOX $(10 \mu \mathrm{M})$ to DOX by GSTA1 and GSTP1 (kind gift from B. Mannervik, Stockholm University) was determined in $0.1 \mathrm{M}$ potassium phosphate buffer (pH 6.5) with $5 \mathrm{mM} \mathrm{GSH}$. For MGST1, $0.1 \mathrm{M}$ potassium phosphate buffer $\mathrm{pH} 6.5$ containing $0.1 \%$ Triton X-100 and 5 mM GSH was used. A standard curve of 0.025, 0.05, $0.1,0.25,0.5,0.1$, and $2.5 \mu \mathrm{M}$ DOX was constructed to convert the increase in fluorescence to a metabolic rate. All measurements were taken in triplicate.

\section{Measurement of GST activity with CDNB}

The specific activity of all purified enzymes was measured in a $100 \mu \mathrm{L}$ cuvette with a Cary 60 UV-visible spectrophotometer (Agilent Technologies, Santa Clara, USA) by following the change in absorbance at $340 \mathrm{~nm}$. For MGST1, $5 \mathrm{mM} \mathrm{GSH}$ (Sigma-Aldrich, St. Louis, MO) and 0.5 mM CDNB (Merck, Darmstadt, Germany) as second substrate were used, respectively, in $0.1 \mathrm{M}$ potassium phosphate buffer $\mathrm{pH} 6.5$ containing $0.1 \%$ Triton X-100. The molar extinction coefficient used for CDNB conjugation was $9.6 \mathrm{mM}^{-}$ ${ }^{1} \mathrm{~cm}^{-1}$ [44]. GSTA1 and GSTP1 were measured with $1 \mathrm{mM} \mathrm{GSH}$ and $1 \mathrm{mM}$ CDNB in $0.1 \mathrm{M}$ potassium phosphate buffer $\mathrm{pH} 6.5$ at $30{ }^{\circ} \mathrm{C}$. The change in absorbance, after correction for the nonenzymatic reaction, was used to calculate the concentration of the active enzyme based on previously published values. All measurements were taken in triplicate, and slopes were fitted using the Cary WinUV software package (Agilent Technologies, Santa Clara, USA).

To study the involvement of MGST1 in drug resistance, the MCF7 cells (human mammary adenocarcinoma) were used. The cells were either transfected with a vector overexpression rat MGST1 or a control vector expressing an antisense sequence against rat MGST1, as described previously [7]. To study GSTA1, V79 cells (Chinese hamster lung fibroblasts) were used. These cells were stably transfected with a vector overexpressing 
GSTA1, and the activity of these was compared with cells expressing control vectors. The GSTA1 containing PCEP4GSTA1 vector is based on the mammalian expression vector pCEP4 (Invitrogen, Inc.) and was used to transfect the V79MZ clone of the V79 cells using a FuGENE6 reagent. The construction of these cells was previously described in Sundberg et al. [45]. These cell lines were cultured in DMEM, supplemented with $10 \%$ fetal bovine serum (FBS), $100 \mathrm{U} / \mathrm{mL}$ penicillin-100 $\mu \mathrm{g} / \mathrm{mL}$ streptomycin (PEST), and 1 mM sodium pyruvate. Additionally $1 \mathrm{mg} / \mathrm{mL}$ Geneticin (G418) or $100 \mu \mathrm{g} / \mathrm{mL}$ hygromycin was added for selection for the MCF7 or V79 cells, respectively. Targeting of TrxR1 by the prodrugs was studied in V79 and GSTA1 overexpressing V79 cells as well as in HEK293 cells (human embryonic kidney). HEK293 cells were cultured in EMEM (Eagle's Minimum Essential Medium, ATCC no. 30-2003). Growth media was supplemented with $10 \% \mathrm{FBS}, \mathrm{PEST}$, and $25 \mathrm{nM}$ sodium selenite. All cells used in this study were cultured in a humidified environment at $37^{\circ} \mathrm{C}$ and with a $5 \% \mathrm{CO}_{2}$ level.

\section{Protein determination and Western Blotting (WB)}

Cells were cultured in a $T-75 \mathrm{~cm}^{2}$ bottles. When the cells reached $70 \%$ confluence, they were washed with $1 \times$ PBS, trypsinized, and resuspended in culture media. The cells were centrifuged for $5 \mathrm{~min}$ at $2000 \mathrm{rpm}$, and the formed pellet was resuspended in lysis buffer (0.1 M potassium phosphate buffer pH 7.5 containing 1\% Triton X-100 and 1\% SDS). The samples were kept on ice during the whole procedure. The cell suspension was transferred into a $1.5 \mathrm{~mL}$ Eppendorf tube. Subsequently, the sonication was performed using the Soniprep 150 MSE, the duration of $10 \mathrm{~s}$, for 3 times at 6 A on ice. The protein determination was performed by using the Coomassie Plus protein assay by Thermo Scientific, Rockford, USA. Twenty micrograms of protein was loaded on a $15 \%$ SDS polyacrylamide gel in a WB system. After running the protein separation, the proteins were transferred to a nitrocellulose membrane, after which they are labeled using a polyclonal rabbit IgG against rat MGST1, which was generated in-house (R. Morgenstern), or against human GSTA1 (1:1000) (Fine chemicals AB Malmö, Sweden), as the primary antibody. As secondary antibody, horseradish peroxidase labeled goat antirabbit IgG obtained from DakoCytomation, Glostrup, Denmark, was used. The blots were developed by using Amersham EL Western blotting reagents obtained from GE Healthcare, Buckinghamhire, UK.

\section{GST Activity in isolated microsomes and total cell lysate}

MGST1 activity was determined in isolated microsomes. The microsomes were prepared by centrifuging the cells at $2000 \mathrm{rpm}$ for $5 \mathrm{~min}$ and resuspended in distilled water containing $0.25 \mathrm{M}$ sucrose and sonicated for $2 \times 10 \mathrm{~s}$ at $4 \mathrm{~A}$ on ice. This suspension was further centrifuged for $10 \mathrm{~min}$ at $3000 \mathrm{rpm}$, followed by transferring the supernatant into a new Eppendorf tube. A mixture of $0.8 \mathrm{M} \mathrm{CaCl}_{2}$ and $0.5 \mathrm{M} \mathrm{MgCl}_{2}(1: 1$ 
$\mathrm{Mg}-\mathrm{Ca}$ ) was added and incubated for $10 \mathrm{~min}$. The samples were then centrifuged for 10 min at $7000 \mathrm{rpm}$, after which the cytosolic fraction in the supernatant was removed. The pellet, containing the microsomes was resuspended in $0.15 \mathrm{M}$ Tris- $\mathrm{HCl}$ buffer $(\mathrm{pH} 8$, containing $1 \mathrm{mM}$ EDTA) and an additional $20 \mu \mathrm{L} \mathrm{Mg-Ca} \mathrm{mixture} \mathrm{was} \mathrm{added.}$ Subsequently the samples were centrifuged for $10 \mathrm{~min}$ at $13000 \mathrm{rpm}$, and the pellet was resuspended in $0.25 \mathrm{M}$ sucrose (in distilled water containing $1 \mathrm{mM}$ EDTA, pH 7.5). The samples were kept on ice during the complete procedure. All centrifugation steps were performed at $4{ }^{\circ} \mathrm{C}$.

The activity of GSTA1 was measured in total cell lysate from GSTA1 overexpressing V79 cells. The cells were centrifuged at $2000 \mathrm{rpm}$ for $5 \mathrm{~min}$, and the cell suspension was transferred into a $1.5 \mathrm{~mL}$ Eppendorf tube. The cell lysate from the V79 cells was obtained by the usage of a lysis buffer containing $0.1 \mathrm{M}$ potassium phosphate buffer $(\mathrm{pH} 6.5)$ with 1\% Triton X-100. After which, the sonication was performed using the Soniprep 150 MSE for the duration of $10 \mathrm{~s}$, for 3 times at $6 \mathrm{~A}$ on ice. The samples were kept on ice during the complete procedure. Afterward, the protein determination was performed by using the Coomassie Plus protein assay by Thermo Scientific, Rockford, USA.

The enzymatic GST activity of isolated microsomes and total cell lysate was determined in $0.1 \mathrm{M}$ potassium phosphate buffer $\mathrm{pH} 6.5$ containing $0.1 \%$ Triton $\mathrm{X}-100$ and $5 \mathrm{mM}$ GSH. As substrate, $0.5 \mathrm{mM}$ CDNB was used, and the reaction was carried out at room temperature. Absorbance change, representing the product formation rate, was measured at a wavelength of $340 \mathrm{~nm}$, using a single beam, Philips PU 8700 UV/visible spectrophotometer (Philips Scientific and Analytical Equipment, Cambridge, UK). All the enzymatic activities were corrected for the nonenzymatic reaction.

\section{Short-term toxicity test}

To measure the short-term ( $24 \mathrm{~h}$ ) cytotoxicity of the prodrugs in the cell lines, a MTT ((3-(4,5-dimethylthiazol-2-yl)-2,5-diphenyltetrazolium bromide)) test was used as previously described [7, 46]. Briefly, the cells were seeded at a density of $1 \times 10^{4}$ cells/well for the MCF7 cells and $2 \times 10^{4}$ cells/well for V79 cell in a 96-well plate. After 1 day, the cells were exposed to DOX, DNS-DOX, ANS-DOX, etoposide, ANS-etoposide, or vehicle (DMSO) for $24 \mathrm{~h}$ at various concentrations in DMEM without phenol red. After $24 \mathrm{~h}$ the media was removed, and the cells were incubated in $0.5 \mathrm{mg} / \mathrm{mL} \mathrm{MTT}$ for $4 \mathrm{~h}$ at $37{ }^{\circ} \mathrm{C}$. The formazan crystals formed were dissolved in DMSO. The absorbance was measured with a spectrophotometer at $590 \mathrm{~nm}$ minus reference at $650 \mathrm{~nm}$ [8].

\section{Long-term toxicity test}

The colony forming efficiency (CFE) assay was used as a long-term (7 days) toxicity test to determine the MGST1 overexpressing cells ability to form colonies after the exposure 
to the toxic agents [47]. The control cells and the MGST1 overexpressing MCF7 cells were seeded in a 6-well plate, with a density of 2000 cells per well. Twenty-four hours after seeding, the cells were exposed to DOX or ANS-DOX in serum-free DMEM (SFM) for $3 \mathrm{~h}$ at different concentrations. After the incubation period the SFM was changed to complete medium and refreshed at day 3. After 7 days the media was removed and cells were washed with PBS and analyzed with the CFE test. The cells were fixated with $10 \%$ formaldehyde for $20 \mathrm{~min}$ and stained with $0.01 \%$ crystal violet for $5 \mathrm{~min}$. Colonies were counted using a light-microscope (a colony $\geq 16$ cells). Since the GSTA1 overexpression V79 cells do not form proper colonies we used the MTT test to monitor the long-term effects of the drugs on these cells. These MTT tests were performed in the following manner: 200 cells per well were seeded on day 0 in complete medium in a 96-well plate; subsequently, on day 2 the cells were exposed to DOX or ANS-DOX, in $\mathrm{SFM}$ for $3 \mathrm{~h}$ at different concentrations. Whereupon the medium was changed to complete medium, and the MTT assay was performed on day 7 as described above.

\section{Analysis of the uptake and activation of ANS-DOX in GSTA1 overexpressing cells}

The vector control cells and GSTA1 overexpressing cells were seeded in 96-well plates. After $24 \mathrm{~h}$ the cells were exposed to either $20 \mu \mathrm{M}$ DOX or ANS-DOX for 15, 30, 60, and $120 \mathrm{~min}$. After the exposure cells were incubated with $40 \mathrm{ng} / \mathrm{mL}$ Hoechst for $30 \mathrm{~min}$ to stain the nuclei and subsequently fixated in $2 \%$ ice-cold paraformaldehyde for $10 \mathrm{~min}$ in RT. The wells were covered with PBS, and fluorescence was measured using the Operetta High Content Imaging System. For cell quantification, seven fields of view for each cell culture well, covering edges and center, were recorded in bright field and two channels for fluorescence detection of DOX (excitation, $500 \mathrm{~nm}$; emission, $595 \mathrm{~nm}$ ) and Hoechst (excitation, $380 \mathrm{~nm}$; emission, $445 \mathrm{~nm}$ ). The excitation and exposure times were fixed for each channel, with all samples analyzed with the same settings. Determinations of fluorescence signals were subsequently performed using the Columbus (PerkinElmer) and Excel (Microsoft) computer programs.

\section{Activation of DNA damage signaling}

The activation of DNA damage signaling in cells exposed to ANS-DOX and DOX was investigated by WB. GSTA1 overexpressing and control cells were exposed to either 0.2 $\mu \mathrm{M}$ DOX or $2 \mu \mathrm{M}$ ANS-DOX for different time periods or for $3 \mathrm{~h}$ with the different concentrations. After incubation with the drugs at indicated time-points or concentrations, the cells were washed with PBS and lysed in $0.1 \mathrm{M}$ potassium phosphate buffer pH 7.5 containing 1\% Triton X-100, 1\% SDS, and phosphatase and protease inhibitors from Roche. Protein samples were run on a $12 \%$ SDS polyacrylamide gel in a WB system. The proteins were then transferred to a nitrocellulose membrane. For the primary antibodies phospho-histone H2AX (Ser139) antibody 1:500 (Cell 
Signaling), phospho-p53 (Ser15) antibody 1:1000 (Cell signaling), and Cdk2 (M2) (Santa Cruz) antibody as loading control 1:1000 were used. As a secondary antibody the horseradish peroxidase labeled goat antirabbit IgG (DakoCytomation) was used. Development of the blots was done via using Amersham EL Western blotting reagents (GE Healthcare).

\section{Comet assay}

The levels of DNA damage were measured after $3 \mathrm{~h}$ exposure to either ANS-DOX ( 1 and $5 \mu \mathrm{M})$ or DOX $(0.02$ and $0.1 \mu \mathrm{M})$. The alkaline version of the comet assay was performed as described previously by Jarvis et al.[48] At least 50 comets were scored per treatment performed in triplicates using a Leica DMLB fluorescent microscope and Comet Assay 3 (Perceptive Instruments Ltd., Haverhill, UK).

\section{GSH measurements and sensitization to DOX upon GSH reduction by BSO}

GSTA1 overexpressing V79 cells were seeded in a 6-well plate the day before exposure to either $5 \mu \mathrm{M}$ ANS-DOX or DNS-DOX. After 2 or $6 \mathrm{~h}$, respectively, cells were washed with PBS and incubated on ice for 30 min with KPE lysis buffer (containing $0.1 \mathrm{M}$ potassium phosphate buffer $\mathrm{pH}$ 7.5, $11.2 \mathrm{mM}$ EDTA, and 1\% Triton X-100). Cells were scraped and centrifuged at $14000 \mathrm{rpm}$ for $10 \mathrm{~min}$ at $4{ }^{\circ} \mathrm{C}$. Sulfosalicylic acid (1.3\%) was added, and samples were stored at $-80{ }^{\circ} \mathrm{C}$ until further usage. GSH content was determined as previously described by Rahman et al.[49] Protein concentrations were determined by using the Bradford assay (BioRad).

To study DOX sensitization upon a reduction in GSH levels, V79 control cells were seeded at a density of $2.5 \times 10^{3}$ cells/well in a 96-well plate and allowed to attach overnight. Subsequently, cells were exposed to $250 \mu \mathrm{M}$ BSO (dl-buthionine-sulfoximine, Sigma-Aldrich) to reduce the GSH content. After $24 \mathrm{~h}$ medium was replaced with DOX containing medium and incubated for another $24 \mathrm{~h}$ at $37{ }^{\circ} \mathrm{C}, 5 \% \mathrm{CO}_{2}$. The medium was removed and a MTT assay was performed as described previously [8].

\section{In vitro characterization of TrxR1 inhibition}

Rat TrxR1 and human Trx1 were recombinantly produced and purified essentially as described previously [50, 51]. TrxR1 activity was measured in TE buffer (50 mM Tris, pH 7.5, $2 \mathrm{mM}$ EDTA) using $250 \mu \mathrm{M}$ NADPH as well as either wild-type Trx1/insulin or juglone (5-OH-1,4-naphthoquinone) as substrates. In contrast to the Trx1 coupled reduction of insulin, which requires an intact Sec-residue in the C-terminal active site, redox-cycling with juglone is essentially Sec-independent but requires an intact $\mathrm{N}$ terminal active site. The behavior of the enzyme, when targeted by an inhibitor, toward 
both substrates can be used to indicate whether an inhibitor preferentially targets the Sec-residue, while leaving the rest of the enzyme essentially intact [52-54].) To assess Cterminal Sec-dependent activity of TrxR1, $20 \mathrm{nM}$ recombinant enzyme was incubated with either $10 \mu \mathrm{M}$ DNS-DOX or ANS-DOX for $10 \mathrm{~min}$ at room temperature in the presence or absence of $250 \mu \mathrm{M}$ NADPH. Subsequently, $10 \mu \mathrm{M}$ wild-type Trx1 and 160 $\mu \mathrm{M}$ insulin (and $250 \mu \mathrm{M}$ NADPH to the samples where it is not present) were added, and the reaction was followed by measuring NADPH consumption via a change in absorbance at $340 \mathrm{~nm}$. Sec-independent activity was studied by incubating $20 \mathrm{nM}$ of recombinant TrXR1 with either $10 \mu \mathrm{M}$ DNS-DOX or ANS-DOX for $10 \mathrm{~min}$ at room temperature in the presence of $250 \mu \mathrm{M} N A D P H$, upon which $40 \mu \mathrm{M}$ juglone was added and the reaction was followed at $340 \mathrm{~nm}$. To study whether the products of the converted prodrugs (free doxorubicin, sulfur dioxide, or the acetylmononitro/dinitro benzene moiety conjugated to GSH, see Fig. 1) can target TrxR1, $10 \mu \mathrm{M}$ DNS-DOX was incubated with $5 \mathrm{mM} \mathrm{GSH}$ and 50 nM GSTA1 for 30 min. Subsequently, 20 nM TrxR1 and $250 \mu \mathrm{M}$ NADPH were added, and the mixture was incubated for 10 more minutes. Finally, $10 \mu \mathrm{M}$ wild-type Trx1 and $160 \mu \mathrm{M}$ insulin were added, and the reaction was followed at $340 \mathrm{~nm}$.

\section{TrxR1 activity in cell lysates}

A total of $2 \times 10^{6}$ HEK293, V79, or GSTA1 overexpressing V79 cells were seeded per dish ( $10 \mathrm{~cm}$ ). Approximately $48 \mathrm{~h}$ (HEK293 cells) or 24 h (V79 and GSTA1 overexpressing cells) after seeding, cells were exposed to the compounds as indicated for $3 \mathrm{~h}$, harvested by trypsinization, and washed in cold PBS. The samples were centrifuged at $900 \mathrm{~g}$ for 5 min, and the resulting cell pellet was resuspended in lysis buffer (50 mM Tris, pH 7.5, 2 mM EDTA, 1\% Triton X-100, and phosphatase and protease inhibitors from Roche). After a 30 min incubation on ice, the cell extracts were cleared by centrifugation at $16,000 \mathrm{~g}$ for $20 \mathrm{~min}$. Protein concentrations of the supernatants were determined using the Bradford reagent (Bio-Rad). Cellular TrxR1 activity was subsequently determined using a modified version of the earlier described end-point insulin assay [55]. Briefly, total cellular protein (20 $\mu \mathrm{g}$ for HEK293 cells or $15 \mu \mathrm{g}$ for V79 and GSTA1 overexpressing cells) was incubated with $20 \mu \mathrm{M}$ wild-type Trx1 in the presence of $160 \mu \mathrm{M}$ insulin and $250 \mu \mathrm{M}$ NADPH using TE buffer for $20 \mathrm{~min}$ at $37{ }^{\circ} \mathrm{C}$ in a total volume of $50 \mu \mathrm{L}$. Samples without additional Trx 1 in the reaction buffer served as a background control. The reaction was stopped by addition of $200 \mu \mathrm{L}$ of $6 \mathrm{M}$ guanidine- $\mathrm{HCl}$ (Acros Organics) with $1 \mathrm{mM}$ DTNB. Absorbance was measured at $412 \mathrm{~nm}$ in a $100 \mu \mathrm{L}$ cuvette using a Cary 60 UV-visible spectrophotometer (Agilent Technologies, Santa Clara, USA). Data were plotted as percentage of untreated control after background subtraction. 


\section{Statistical analysis}

The cytotoxicity study data were analyzed by performing an F-test for equal variances, followed by a two-tailed student t test. The GST activity, Comet assay, and TrxR1 inhibition data were analyzed by using a two-tailed student t test. GraphPad prism software windows version 5.03 (GraphPad Software, 2009, California, USA) was employed in order to perform nonlinear regression analysis, and sequentially, curve comparisons were performed by using an extra sum F-test.

\section{Results}

\section{MGST1, GSTP1, and GSTA1 catalyzed conversion of DNS-DOX and ANS-DOX to DOX}

The modifications in DOX that yield ANS-DOX and DNS-DOX conveniently quench the fluorescence intensity of DOX 10- to 20-fold (Figure 2A). Taking advantage of the fluorescence increase upon conversion to the more fluorescent DOX by MGST1, GSTP1, and GSTA1, we measured the specific activity for each enzyme. Catalytic rates were in general agreement with previous results for DNS-DOX (Fig. 2B)[8]. Oxidative activation of MGST1 or loss of activity of GSTP1 upon storage account for the differences observed. GSTA1, which was not yet tested with DNS-DOX, showed a very high turnover of $13780 \pm 120 \mathrm{nmol} / \mathrm{min} / \mathrm{mg}$ that by far exceeded the activity of MGST1 (463 $\pm 7 \mathrm{nmol} / \mathrm{min} / \mathrm{mg}$ ) and GSTP1 (18.7 $\pm 2 \mathrm{nmol} / \mathrm{min} / \mathrm{mg})$. Subsequently we measured the turnover of all enzymes with the DOX derivative ANS-DOX. Having a less electron withdrawing acetyl group, as is also reflected in the difference of the nonenzymatic conversion rates (Fig. 2C), ANS-DOX showed 10 to 30 times lower conversion rates for all enzymes compared to DNS-DOX as expected [56]. ANS-DOX was thus activated with a rate of $38.7 \pm 0.2 \mathrm{nmol} / \mathrm{min} / \mathrm{mg}$ by MGST1 and $493 \pm 3 \mathrm{nmol} / \mathrm{min} / \mathrm{mg}$ by GSTA1, whereas no activity could be measured for GSTP1 under the experimental conditions (Fig. 2B). The presence of Triton X-100, furthermore, did not alter the ratio of nonenzymatic reactivity for the compounds drastically, suggesting that solubility and lipophilicity is not a major factor (Fig. 2C). A conversion rate of the etoposide based prodrug ANS-etoposide by the purified enzymes was not determined as the molecule lacks fluorescence. 
A Emission spectra-0.1M Phosphate buffer

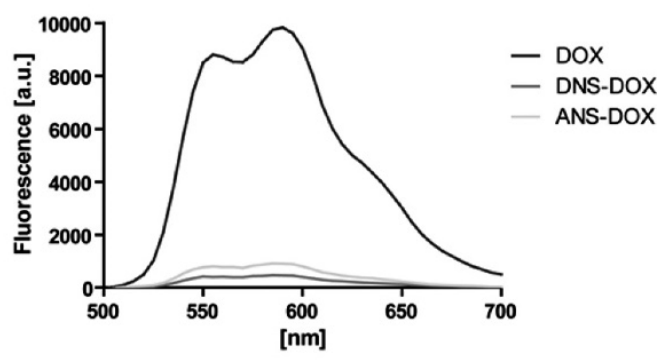

B

\begin{tabular}{l|ccc}
\multirow{2}{*}{ Enzymes } & \multicolumn{3}{|c}{ [nmol/min/mg] } \\
\cline { 2 - 4 } Substrates & MGST1 & GSTP1 & GSTA1 \\
\hline DNS-DOX & $463 \pm 7$ & $18.7 \pm 0.2$ & $13780 \pm 120$ \\
\hline ANS-DOX & $38.7 \pm 0.2$ & N.D. & $493 \pm 3$ \\
CDNB & $7850 \pm 230$ & - & -
\end{tabular}

C

\begin{tabular}{l|cc}
\multirow{2}{*}{ Substrates } & \multicolumn{2}{|c}{$k_{\text {noncat }}\left[\mathrm{M}^{-1} \mathrm{~s}^{-1}\right]$} \\
\hline wNithout $0.1 \%$ Triton X-100 & with $0.1 \%$ Triton X-100 \\
\hline ANS-DOX & $(9.3 \pm 0.4) \times 10^{-1}$ & $(1.1 \pm 0.1) \times 10^{-1}$ \\
& $(6.6 \pm 0.2) \times 10^{-2}$ & $(2.3 \pm 0.7) \times 10^{-2}$
\end{tabular}

Figure 2. Emission spectra of DOX and its derivatives, second order rate constants for the nonenzymatic ANSDOX and DNS-DOX conversion, and the catalyzed conversion of ANS-DOX, DNS-DOX, and CDNB by MGST1, GSTP1, and GSTA1.

A. Emission spectra of DOX (dark gray), DNS-DOX (medium gray), and ANS-DOX (light gray) in $0.1 \mathrm{M}$ potassium phosphate buffer pH 6.5 using excitation at $480 \mathrm{~nm}$. B. Specific activities of MGST1, GSTP1, and GSTA1 were determined using $5 \mathrm{mM}$ GSH and $10 \mu \mathrm{M}$ ANS-DOX, $10 \mu \mathrm{M}$ DNS-DOX, or $0.5 \mathrm{mM}$ CDNB in $0.1 \mathrm{M}$ potassium phosphate buffer $\mathrm{pH} 6.5$ (without or with $0.1 \%$ Triton X-100 in the case of MGST1). The specific CDNB activities of GSTP1 and GSTA1 were compared to literature values to determine the amount of active enzyme in the preparations, which in turn was used to calculate the specific activity toward ANS-DOX and DNS-DOX. N.D., not detectable. Values are means \pm SEM $(n=3)$. (C) Second-order rate constants for the nonenzymatic reaction (knoncat) were obtained in $0.1 \mathrm{M}$ potassium phosphate buffer $\mathrm{pH} 6.5$ (without or with $0.1 \%$ Triton X-100), using $5 \mathrm{mM} \mathrm{GSH}$ as well as $10 \mu \mathrm{M}$ ANS-DOX or DNS-DOX, respectively. Values are means $\pm \operatorname{SEM}(n=3)$.

\section{GSTA1 overexpression amplifies ANS-DOX induced toxicity but has no effect on DOX and DNS-DOX toxicity}

Based on our finding that purified GSTA1 catalyzed the conversion of DNS-DOX much more efficiently (30-fold) than MGST1 (see Fig. 2B), we studied the effect of this DOX derivative and pure DOX in GSTA1 overexpressing cells. Overexpression was confirmed by WB and activity measurements (Fig. 3A). Using a short-term (24 h; MTT) as well as 
long-term (7 days) viability test, no difference in DOX toxicity could be seen between V79 control and GSTA1 overexpressing cells (Fig. 3B). The same behavior was surprisingly observed when exposing cells to DNS-DOX, which showed no GSTA1dependent toxicity in a short-term viability test (24 h; MTT) despite its high catalytic efficiency (13780 $\pm 120 \mathrm{nmol} / \mathrm{min} / \mathrm{mg}$ ) (Fig. 3C). In contrast, the less reactive ANS-DOX variant (493 $\pm 2 \mathrm{nmol} / \mathrm{min} / \mathrm{mg}$ ) induced toxicity to a significantly greater extent in GSTA1 overexpressing compared to control cells in short-term (24 h; MTT) as well as long-term viability test (7 days) (Fig. 3D).

$\mathbf{A}$

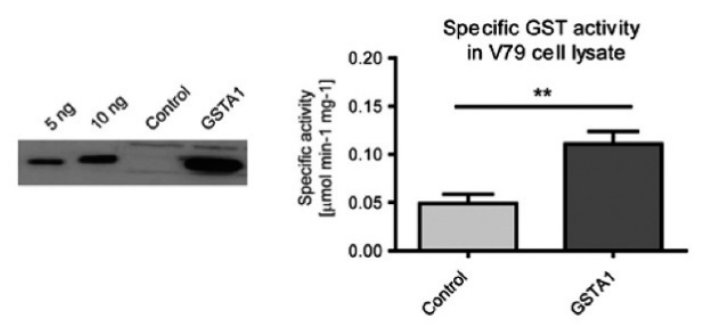

B

DOX (short-term MTT)

DOX (long-term MTT)
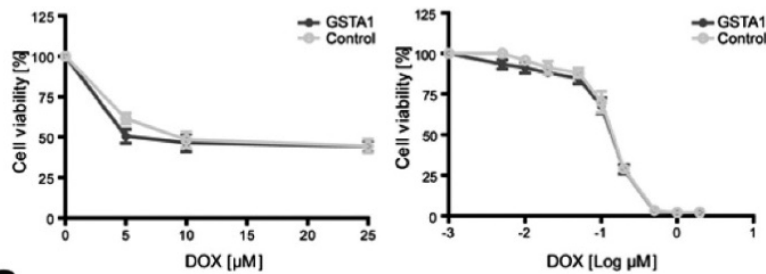

C

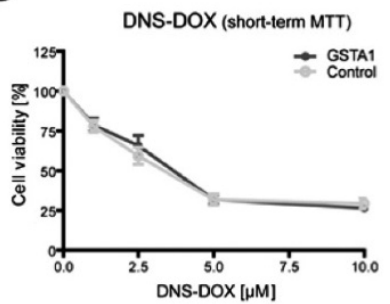

D

ANS-DOX (short-term MTT)
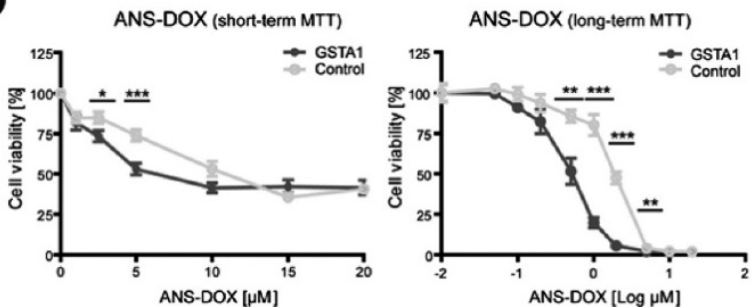

Figure 3. Characterization of GSTA1 overexpressing cell lines and their response to DOX, DNS-DOX, and ANSDOX treatment.

A. Left panel: Western blot of GSTA1. Lanes 1-2: purified human GSTA1; 5 and $10 \mathrm{ng}$. Lane 3: vector control 
cell line. Lane 4: GSTA1 overexpressing cell line. Right panel: Specific GST activity in cell extracts from GSTA1 overexpressing and control V79 cells. Activity was measured using $0.5 \mathrm{mM}$ CDNB and $5 \mathrm{mM} \mathrm{GSH}$. Values are means \pm SEM $\left(n=3 ;{ }^{* * P} \leq 0.01\right)$. B-D Cellular toxicity of (B) DOX $(\log (-3) \mu M$ corresponds to $0 \mu M$ DOX treatment), (C) DNS-DOX, and (D) ANS-DOX for short-term (24 h) or long-term toxicity assay (7 days) with indicated doses determined with MTT assay. GSTA1 overexpressing V79 cells (dark gray/GSTA1) and the vector control (light gray/control). Data are from at least three independent experiments done in triplicates ( $\mathrm{n}$ $\geq 9$ ) (means $\pm S E$ ) ${ }^{*} P \leq 0.05,{ }^{* * P} \leq 0.01$, and $\left.{ }^{* * *} P \leq 0.001\right)$. $\log (-2) \mu \mathrm{M}$ corresponds to $0 \mu \mathrm{M}$ DOX treatment. Curve comparison long-term toxicity assay: DOX ( $p=0.42)$; ANS-DOX ( $p<0.0001)$.

\section{Uptake, conversion, and DNA damage in GSTA1 overexpression cells after ANS- DOX exposure}

In order to further investigate whether ANS-DOX is converted to DOX in a GSTA1dependent manner within the cells we exposed GSTA1 overexpressing and control V79 cells to ANS-DOX as well as DOX and measured the final cellular DOX content at various time points. For this we exploited that the conversion of ANS-DOX yields more fluorescent DOX, which was detected using the Operetta High content imaging system. Using DOX as a control we could see a similar steady and rapid increase in cellular levels in both control cells and GSTA1 overexpressing cells (Fig. 4A). This further indicates that a cellular uptake of DOX (and also ANS-DOX) does not depend on the expression level of GSTA1, but that the different toxicity profiles are indeed dependent on GSTA1 catalysis. Exposing cells to ANS-DOX we could clearly see an GSTA1 and timedependent increase in nuclear DOX fluorescence showing that ANS-DOX is intracellularly converted to DOX by GSTA1 (Fig. 4A). Visualizing single cells, it becomes clear that the control cells at early time points show a predominantly low cytosolic fluorescence (indicative of uncleaved prodrug) with nearly no nuclear DOX, whereas the GSTA1 overexpressing cells display a very strong nuclear DOX fluorescence (Figure 4B; compare merge control and GSTA1 overexpression). A similar pattern could be seen with MGST1 overexpressing cells (data not shown), but to a much lower extent, as expected, since GSTA1 has a much higher ANS-DOX conversion rate compared to MGST1 (Fig. 2B). 
A

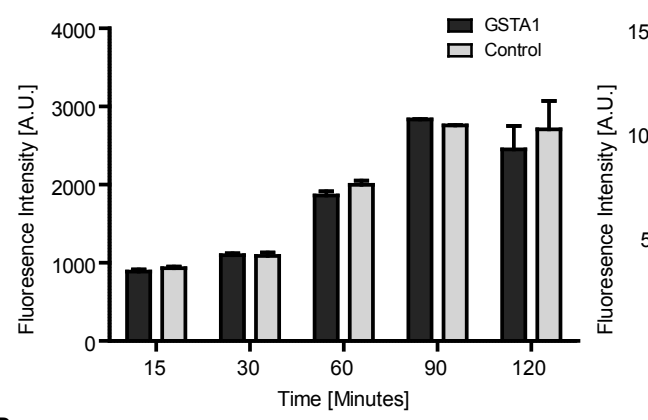

B
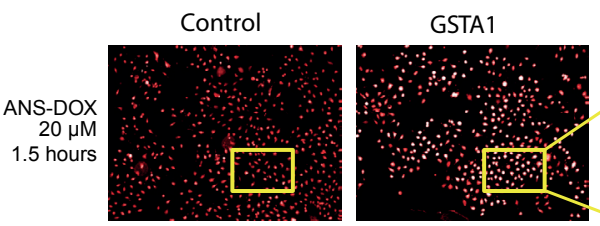

Control
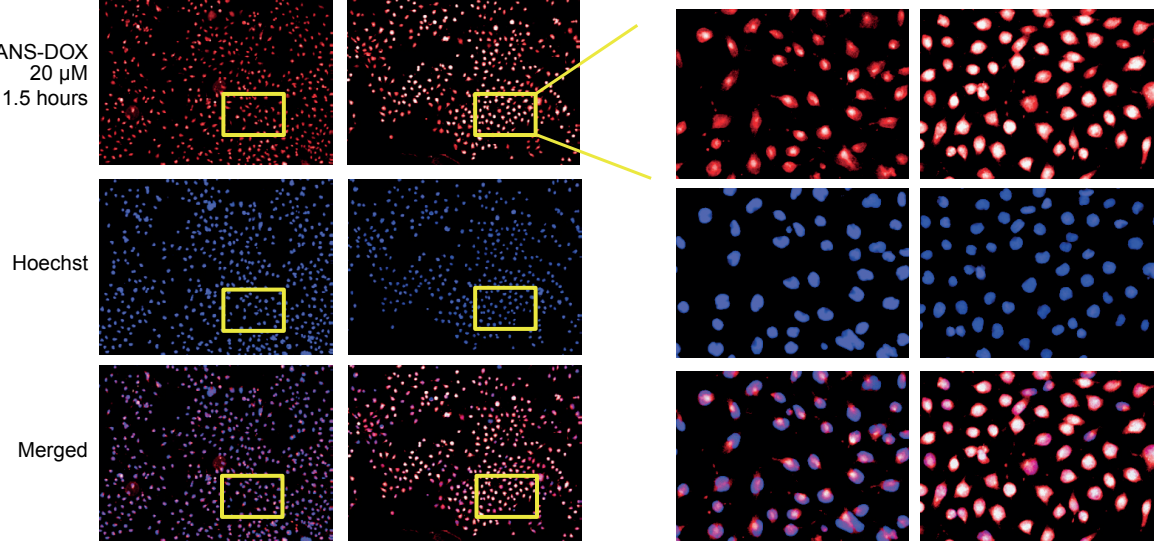

Figure 4. Cellular uptake of DOX and ANS-DOX in GSTA1 overexpressing cells.

A. Representative figure of fluorescent intensity of DOX in the nuclei of cells treated with either $20 \mu \mathrm{M}$ DOX or $20 \mu \mathrm{M}$ ANS-DOX for the indicated time periods in GSTA1 overexpressing (dark gray) and vector control (light gray) cells $(n=3$; mean \pm SEM). B. Representative fluorescence microscopy pictures of GSTA1 overexpression and V79 control cells, treated with $20 \mu \mathrm{M}$ ANS-DOX for $120 \mathrm{~min}$. Upper panel: DOX fluorescence. Middle panel: Hoechst staining of the nucleus. Lower panel: Merge images.

\section{DNA damage in GSTA1 overexpression cells after ANS-DOX exposure}

Looking at DNA damage we noted that GSTA1 overexpression protected the cells somewhat from DOX induced DNA damage in a time-dependent manner when compared to the control cells as seen by expression of the DNA damage markers $\mathrm{p}$ Histon-H2AX and p-p53. This effect could not be seen with ANS-DOX treatment, where control as well as GSTA1 overexpression cells showed significant induction of DNA damage markers (Fig. 5A, left panel). Notable, the GSTA1-dependent protection seems to somewhat disappear at higher concentration of DOX (Fig. 5A, right panel). This furthermore goes in line with the alkaline comet assay (Fig. 5B, right panel), confirming 
that the cells having GSTA1 overexpression displayed less DNA damage compared to control cells at $0.2 \mu \mathrm{M}$ DOX, but not at $0.1 \mu \mathrm{M}$ DOX treatment. GSTA1 has potentially a small protective effect toward DOX, which, however, disappears at higher concentrations where GSTA1 might be overwhelmed. By exposing GSTA1 overexpression cells to ANSDOX, on the other hand, higher levels of DNA damage were observed compared to control cells (Fig. 5B) in line with the cell viability assays (see Fig. 3), the ANS-DOX to DOX conversion (see Figure 4), and also the DNA damage markers (see Fig. 5A).
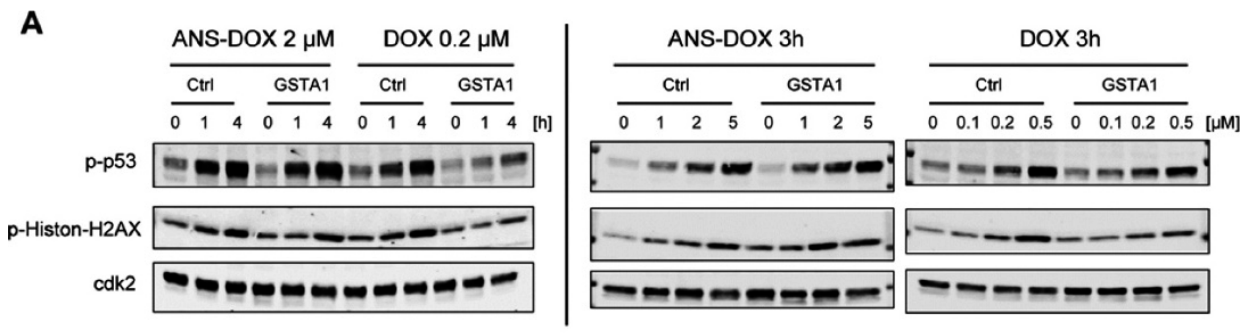

B Comet assay - ANS-DOX
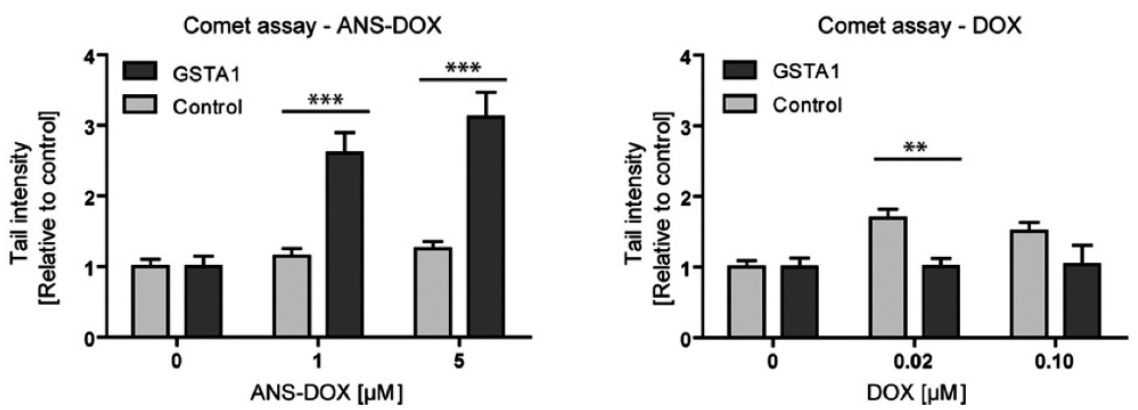

Figure 5. DNA damage in GSTA1 overexpressing cells after ANS-DOX and DOX exposure.

A. Activation of DNA damage signaling was assessed by phosphorylation of p53 at Ser15 and Histon- $\mathrm{\gamma H} 2 \mathrm{AX}$ at Ser139 using Western blot. Cdk2 was used as a loading control. GSTA1 overexpressing and vector control cells were treated with $0.2 \mu \mathrm{M}$ DOX or $2 \mu \mathrm{M}$ ANS-DOX for the indicated time periods or for $3 \mathrm{~h}$ with the indicated concentration. B. DOX and ANS-DOX mediated DNA damage in GSTA1 overexpressing (light gray) and vector control cells (dark gray) were determined after a $3 \mathrm{~h}$ exposure by comet assay. Experiments were performed in triplicate, and data points represent means \pm SEM ${ }^{* *} p<0.01, * * * p<0.001$ as compared with control levels by using a two-tailed student $t$ test.

\section{ANS-DOX reverses the MGST1-dependent resistance against DOX}

MGST1 was previously shown to promote resistance to DOX and to be a potential target in the activation of DOX based prodrugs, highlighting it as a suitable target to further validate ANS-DOX as a potential prodrug [8]. For this we utilized control MCF7 cells, known to have low endogenous levels of GSTs and MCF7 cells engineered to overexpress MGST1 (Fig. 6A) [57]. MCF7 cells are known to easily overexpress features associated with multidrug resistance, particularly using DOX as a model drug. Even if the cells used in this study were not especially adapted to generate a resistant phenotype, 
they still displayed high resistance to DOX, suggesting that they employ several mechanisms to counter DOX induced cell death [27, 58-62]. Resistance to DOX was furthermore significantly promoted by MGST1 overexpression compared to control cells, in agreement with previous studies [8, 9]. This protective effect was indeed observed in short-term ( $24 \mathrm{~h}$ ) exposure studies. However, only marginal effects were found in long-term follow-up CFE experiments (7 days) (Fig. 6B). Total reversal of MGST1-dependent resistance against DOX was shown in cells exposed to ANS-DOX even though no further toxicity was induced in MGST1 overexpressing cells compared to cells with low MGST1 (compare Fig. 6B,C).
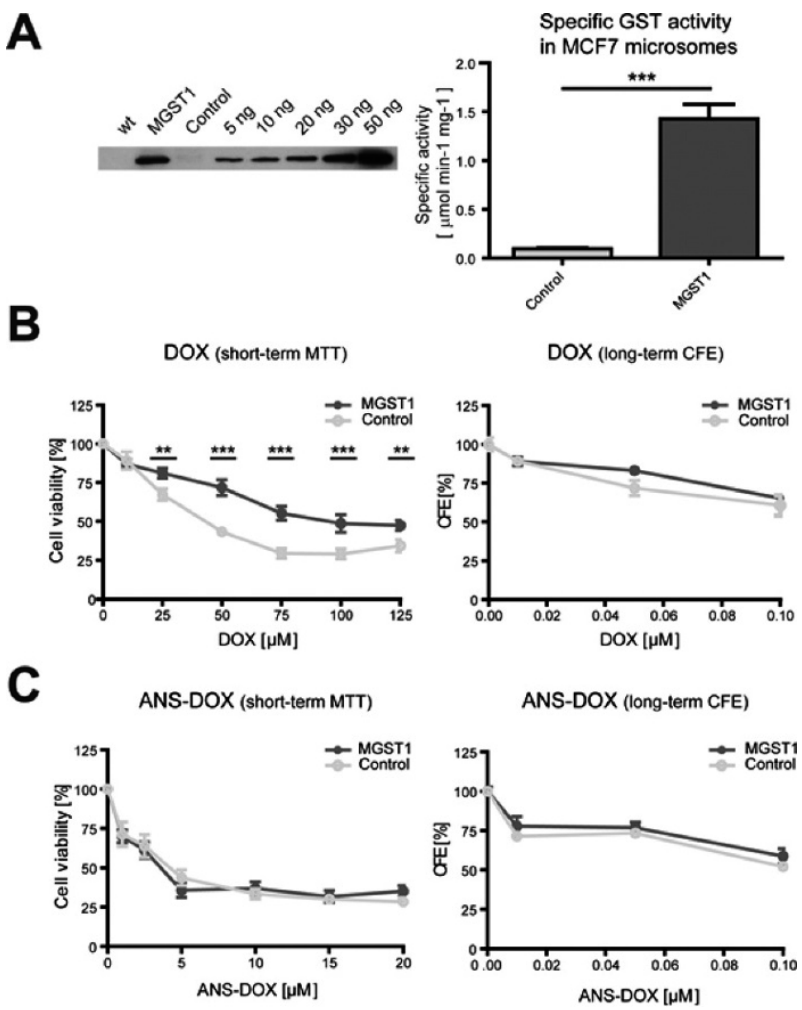

ANS-DOX (long-term CFE)

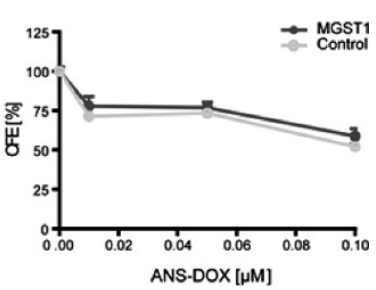

Figure 6. Characterization of MGST1 overexpressing cell lines and their response to DOX and ANS-DOX treatment.

A. Left panel: Western blot of MGST1. Lane 1: cell lysate of wildtype MCF7 cells. Lane 2: MCF7 cells overexpressing MGST1. Lane 3: MCF7 cells containing vector control. Lane 4 until lane 9: purified rat MGST1 enzyme; 5-50 ng. Right panel: Specific GST activity in isolated microsomes from MGST1 overexpressing and control MCF7 cells. Activity was measured using $0.5 \mathrm{mM}$ CDNB and $5 \mathrm{mM} \mathrm{GSH}$. Values are means \pm SEM $(n=$ 3; *** $\mathrm{p} \leq 0.001)$. B-C. Cellular toxicity of (B) DOX or (C) ANS-DOX after $24 \mathrm{~h}$ analyzed with short-term MTT assay (left) or long-term colony forming efficiency (CFE) assay (right; 7 days) with indicated doses. Data are derived from at least three independent experiments done in triplicate (mean \pm SEM, $n \geq 9, * * p \leq 0.01, * * * p \leq$ 0.001). CFE panels are representative data from one experiment measured in triplicate. MGST1 overexpressing MCF7 cells (dark gray/MGST1) and the vector control (light gray/control). 


\section{Prodrug ANS-Etoposide could overcome Etoposide-dependent resistance shown in GSTA1 overexpressing cells}

In addition to DOX and its derivatives we included etoposide and an etoposide based prodrug that was modified with a nitro acetyl-benzene moiety conjugated to the etoposide (ANS-etoposide) through a sulfonic acid ester linkage. In opposition to DOX treatment (see Fig. 3B), cells overexpressing GSTA1 showed significant resistance against etoposide. The GSTA1-dependent resistance against etoposide was reversed when exposing cells to ANS-etoposide, suggesting that the prodrug acts either independently of GSTA1 or that the enzyme-dependent activation of the drug cancels out the protection warranted by GSTA1 overexpression (Fig. 7A). It was previously noted that the $\mathrm{V} 79$ cells are generally more sensitive toward both DOX and etoposide than the MCF7 cells [63]. That was shown also here with both etoposide and ANSetoposide. However, MGST1 overexpression seemed to promote a weak ANSetoposide induced toxicity compared to control cells and in relation to the parental etoposide (Fig. 7B). Since etoposide is not fluorescent as DOX, no GST-dependent conversion rates could be obtained.

A

Etoposide (short-term MTT)
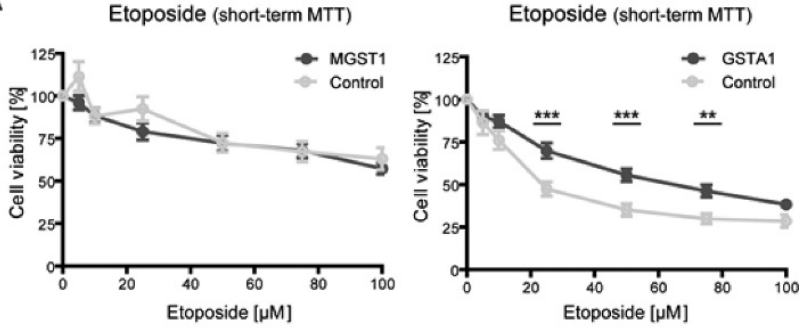

B
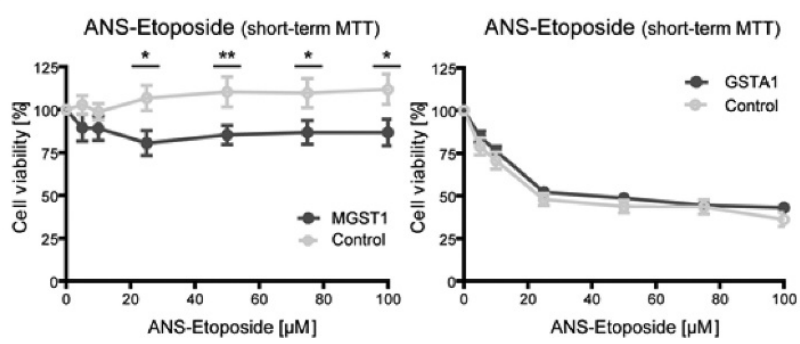

Figure 7. Etoposide and ANS-etoposide toxicity in GSTA1 and MGST1 overexpressing cells.

A-B. Cellular toxicity of (A) etoposide and (B) ANS-etoposide analyzed with the MTT assay after short-term 24 $h$ exposure with indicated doses, in at least three independent experiments measured in at least triplicate (mean \pm SEM, $n \geq 9, * p \leq 0.05, * * p \leq 0.01$, and ${ }^{* * *} p \leq 0.001$ ). Left panel: MGST1 overexpressing MCF7 cells (dark gray/MGST1) and the vector control (light gray/control). Right panel: GSTA1 overexpressing V79 cells (dark gray/GSTA1) and the vector control (light gray/control). 
Reduction of GSH Levels through Prodrug Activation and Sensitization to DOX

To study whether activation of the prodrugs leads to GSH depletion and thus sensitization to DOX, we treated GSTA1 overexpressing V79 cells with $5 \mu \mathrm{M}$ ANS-DOX and DNS-DOX and measured the total GSH content after 2 and $6 \mathrm{~h}$ of exposure. The early time point showed only a minor reduction of the GSH levels, which were replenished again at $6 \mathrm{~h}$ (Fig. 8A). This is not surprising as in this setup a maximum of $2.5 \mathrm{nmol}$ prodrug $(5 \mu \mathrm{M})$ could only deplete about $50 \%$ of the approximately $5 \mathrm{nmol}$ total GSH (500 $\mu \mathrm{g}$ total protein in the sample with a GSH content of about $10 \mathrm{nmol} / \mathrm{mg}$ protein). Nonetheless, this highlights that prodrug uptake and activation is a rapid process involving a decrease of the GSH pool. Furthermore, in the scenario of a high accumulation within the tumor, a more pronounced GSH reduction might contribute to the toxicity profile as the cells become sensitized to the activated DOX [64]. To validate this previously reported sensitization through a reduction in GSH we treated V79 cells with BSO and exposed them to DOX. Already a decrease in the GSH levels of about $65 \%$ (Control, $12 \pm 6 \mathrm{nmol} / \mathrm{mg}$ protein, versus BSO treated, $4 \pm 3 \mathrm{nmol} / \mathrm{mg}$ protein) resulted in a significant increase in DOX toxicity (Fig. 8B) and thus suggests that the concomitant reduction in GSH levels may indeed synergize with the activated drug.

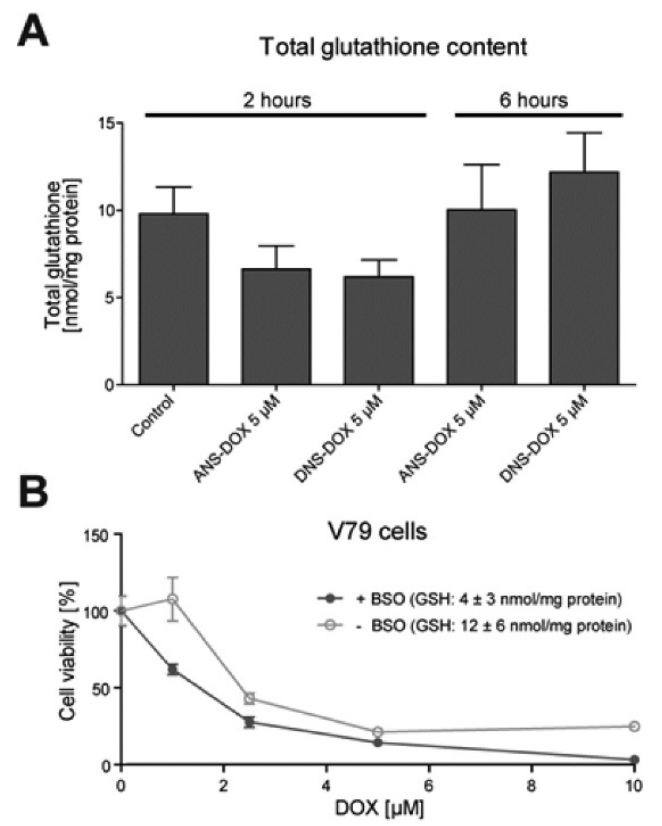

Figure 8. GSH depletion by prodrugs and sensitization to DOX.

A. GSTA1 overexpressing V79 cells were treated with the prodrugs for 2 or $6 \mathrm{~h}$, respectively. Cells were subsequently lysed, and total GSH content was determined (Mean \pm SEM, $n=3$ ). B. Cells were depleted of GSH using BSO and treated with DOX as indicated. GSH levels of control cells, $12 \pm 6 \mathrm{nmol} / \mathrm{mg}$ protein; GSH levels of BSO treated cells, $4 \pm 3 \mathrm{nmol} / \mathrm{mg}$ protein. Cellular toxicity was analyzed with the MTT assay after $24 \mathrm{~h}$ exposure (mean $\pm S E M, n=3$ ). Curve comparison: $p=0.09$. 


\section{Thioredoxin Reductase 1 inhibition as concomitant mechanisms of the prodrugs}

The electrophilic nature of the sulfonamide bond suggests that other intracellular nucleophiles, in addition to GSH, can also be the target of the prodrugs. A prime candidate is the selenoprotein TrxR1, which is the central enzyme in the antioxidant Trx system. Using recombinant TrxR1, DNS-DOX and ANS-DOX could both efficiently inhibit its activity toward Trx1, while having little effect on its ability to redox cycle with juglone (Figure 9A, left panel). Juglone, in contrast to Trx1, does not require an intact Secresidue at the C-terminal $[52,53]$. Inhibition by the prodrugs was furthermore NADPH dependent, suggesting that the reduced, highly nucleophilic and reactive Sec-residue in the C-terminal active site is the prime target (Fig. 9A, right panel). This experiment was performed without a desalting step after a 10 min incubation of $20 \mathrm{nM}$ TrxR1 with 10 $\mu \mathrm{M}$ of DNS-DOX in the presence or absence of NADPH. Subsequently, the remaining DNS-DOX started to competitively inhibit TrxR1 as $10 \mu \mathrm{M} \operatorname{Tr} 1,160 \mu \mathrm{M}$ insulin, and 250 nM NADPH were added to measure the enzyme activity. In contrast to the prodrug, neither DOX, the sulfur dioxide, nor the GSH conjugated acetylmononitro/dinitro benzene moiety, which are the products of prodrug activation via GSH, are inhibitors of TrxR1 (Fig. 9A, right panel). The underlying mechanism thus potentially involves the covalent modification of the Sec-residue by the acetylmononitro/dinitro benzene moiety of the prodrug in a reaction similar to that of the prodrugs with GSH. To study whether TrxR1 is also targeted within the cell we utilized HEK293 cells and treated those with 2 and $10 \mu \mathrm{M}$ of DNS-DOX and ANS-DOX as well as $1 \mu \mathrm{M}$ auranofin. Auranofin is a well-known TrxR1 inhibitor and often used as a standard to compare the efficiency of compounds in targeting cellular TrxR1 [54, 65, 66]. Compared to auranofin, both DNS-DOX and ANS-DOX showed a surprisingly high degree of TrxR1 inhibition even at $2 \mu \mathrm{M}$ (Fig. 9B). However, HEK293 cells with $0.013 \pm 0.001 \mu \mathrm{mol} / \mathrm{min} / \mathrm{mg}$ protein have a rather low GST-dependent CDNB activity compared to V79 cells (V79 control, $0.05 \pm 0.01 \mu \mathrm{mol} / \mathrm{min} / \mathrm{mg}$; GSTA1, $0.11 \pm 0.01 \mu \mathrm{mol} / \mathrm{min} / \mathrm{mg}$; see Fig. 3B). This suggests overall lower GST levels in HEK293 cells and thus slower prodrug activation compared to V79 cells, leaving the prodrugs more time to target TrxR1. To test whether TrxR1 inhibition is limited by prodrug activation via GST/GSH we treated V79 control cells and GSTA1 overexpressing V79 cells with DNS-DOX, ANS-DOX, and auranofin. As expected, TrxR1 inhibition was lower in V79 compared to HEK293 cells and even further lowered by GSTA1 overexpression (Fig. 9C). 
A

B

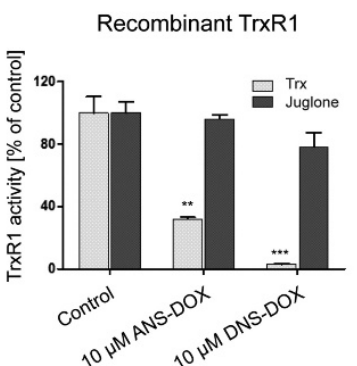

$\overline{\bar{c}}$ TrxR1 activity in HEK293 cells

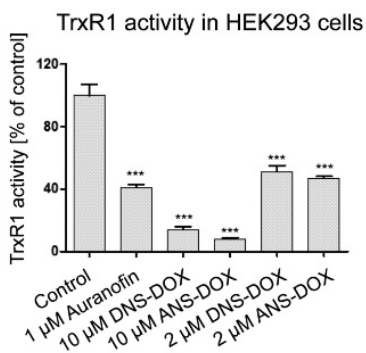

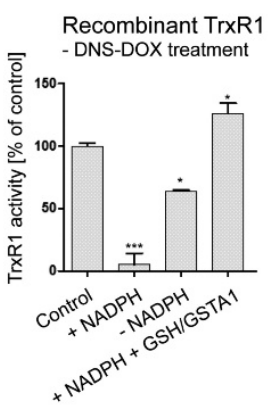

C

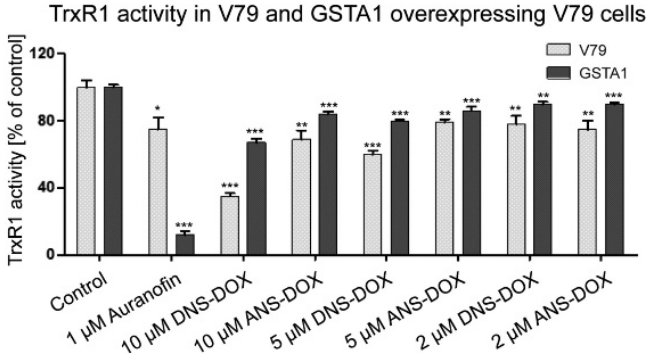

Figure 9. TrxR1 inhibition by DNS-DOX and ANS-DOX.

A. Inhibition of recombinant TrxR1 by auranofin, DNS-DOX, and ANS-DOX. Left panel: In vitro TrxR1 activity. Twenty nanomolar recombinant TrxR1 was incubated with the drugs as indicated for 10 min in the presence of NADPH. Enzyme activity was measured using either Trx1 or juglone as substrate (mean $\pm S E M, n=3$ ). Right panel: In vitro TrxR1 activity. Twenty nanomolar recombinant TrxR1 was incubated with $10 \mu \mathrm{M}$ DNS-DOX either in the presence (+NADPH) or absence (-NADPH) of NADPH for $10 \mathrm{~min}$. Subsequently, enzyme activity was measured using Trx1 as substrate. TrxR1 inhibition by the products of prodrug activation (DOX, SO2, and GSH conjugated acetylmononitro/dinitro benzene moiety) was determined by first incubating $10 \mu \mathrm{M}$ DNSDOX with $5 \mathrm{mM} \mathrm{GSH}$ and $50 \mathrm{nM}$ GSTA1 for $30 \mathrm{~min}$. Subsequently, $20 \mathrm{nM}$ TrxR1 and $250 \mu \mathrm{M}$ NADPH were added for 10 min. Enzyme activity was measured using Trx1 as substrate (mean $\pm \mathrm{SEM}, \mathrm{n}=3$ ). B. Cellular TrxR1 activity. HEK293 cells were treated with the drugs as indicated for $3 \mathrm{~h}$. Enzyme activity was measured in cell extract using a modified form of the Trx1 coupled insulin end point assay. Data are from two independent experiments measured in triplicate (mean \pm SEM, $n=6$ ). C. TrxR1 inhibition in V79 control cells versus GSTA1 overexpressing V79 cells. Cells were treated with the drugs as indicated for $3 \mathrm{~h}$, and enzyme activity was measured in cell extract using a modified form of the Trx1 coupled insulin end point assay. Data are from three independent experiments measured in duplicate (mean \pm SEM, $n=6$ ). ${ }^{*} p \leq 0.05,{ }^{* *} p \leq 0.01$, and ${ }^{* * *} p \leq$ 0.001 as compared with control levels by using a two-tailed student $t$ test. 


\section{Discussion}

The difficulty to selectively target cancer cells and the development of resistance to treatments are among the major problems in current cancer therapy. Additionally, the efficiency of a drug may be limited by a lack of activation or inactivation, poor drug influx or excessive efflux, alterations in expression levels of the drug target, or the activation of adaptive pro-survival pathways [67]. We previously reported that MGST1 and GSTP1 overexpression promotes resistance to DOX but that this resistance could be overcome using the prodrugs MNS-DOX and DNS-DOX, respectively (Fig. 1A) [8]. The major mechanism is the GST catalyzed release of DOX from these prodrugs, leading to selected accumulation and targeting of cells that have this particular GST overexpression, while potentially sparing other cells and minimizing side effects. While expanding our studies to other GSTs in order to further validate this concept we noticed that purified GSTA1 enzyme had an extremely high specific activity and conversion rate with DNS-DOX. Surprisingly, however, despite the efficient release of DOX there was an unexpected lack of cell toxicity augmentation in GSTA1 overexpression cells. We reasoned that there might be an enzyme-dependent "conversion rate window" that is optimal for the cells to be killed selectively by the prodrugs. This hypothesis is supported by our previous study showing that MGST1 overexpression augmented MNS-DOX toxicity while having only small effects on DNS-DOX toxicity despite a 100 times higher conversion rate for DNS-DOX compared to MNS-DOX [8]. A similar behavior was observed in GSTP1 overexpressing cells, where DNS-DOX (converted with $84 \pm 12 \mathrm{nmol} / \mathrm{min} / \mathrm{mg}$ ) toxicity was augmented by GSTP1 overexpression to a greater extent than by MGST1 overexpression, which has a higher conversion rate [8]. Our reasoning is that a limited conversion rate capitalizes more optimal on the overexpression of the particular GST, leading to a stronger GST-dependent accumulation of the converted drug and thus a more selective cell killing. Highly reactive prodrugs, however, might already be converted at rates that are high enough to render a contribution from additional GST overexpression minor. This assumption is further strengthened by a recent study showing that limiting the prodrug reactivity can translate to a prolonged lifetime in the bloodstream and thus to a higher in vivo efficacy. For this, Nandurdikar et al. developed analogues of JS-K, an arylated diazeniumdiolate prodrug, where the nitro group substituents in the aromatic ring were replaced with less electron-withdrawing groups such as cyano or fluoro. Interestingly, a small decrease in reactivity promoted an increased efficacy, whereas too low reactivity resulted in a loss of effect [68].

To further test this hypothesis, we developed a DOX derivative with altered reactivity, 4acetyl-2-nitro-benzenesulfonyl doxorubicin (ANS-DOX), where a nitro group in the para position in DNS-DOX is replaced by an acetyl group (Fig. 1). The less electron withdrawing acetyl group reduced the reactivity about 10-30 times compared to DNS- 
DOX (Fig. 2B). In line with our hypothesis, ANS-DOX toxicity was indeed strongly augmented in a GSTA1-dependent manner despite its lower activity (Fig. 3), which could be further confirmed by the time and GSTA1-dependent increase in nuclear DOX fluorescence (after ANS-DOX conversion) as well as GSTA1-dependent DNA damage (Fig. 4 and 5). A similar behavior could be observed with MGST1, albeit to a smaller extent. ANS-DOX showed hereby a toxicity profile that seems at first independent of the MGST1 status. However, it is likely a combination of two opposing effects that cancel each other out; the MGST1-dependent conversion of ANS-DOX to DOX and MGST1 mediated resistance. Comparing the toxicity profiles of ANS-DOX with the previously studied DNS-DOX and MNS-DOX it becomes apparent that the toxicity of these prodrugs followed the conversion rates inversely [8].

Notably, ANS-DOX induced toxicity in the MCF7 cells at much lower concentrations than DOX in a manner that seems independent of the GST status. This effect was also previously reported for DNS-DOX in the treatment of MCF7 cells [8], suggesting that these modifications alter the properties of DOX in a way that allow it to circumvent multidrug resistance mechanisms present in the MCF7 cells and thus act as a delivery system for the parental drug. A general effect such an increased uptake and altered intracellular distribution due to a change in the lipophilicity of the drug (logP; ANS-DOX: 2.2 vs. DOX: 0.6), might in part explain the behavior. However, this effect was predominant in MCF7 cells and not in the already more sensitive V79 cells in $24 \mathrm{~h}$ treatment experiments, suggesting that the prodrug might avoid one or more drug resistance mechanisms present in the MCF7 cells. A potential mechanism could be the avoidance of transmembrane transporter proteins such as P-glycoprotein (P-gp) [60], membrane multidrug transporters (MRP1), or the breast cancer resistance protein (BCRP)[69],(70) which were previously reported to be important for DOX resistance in MCF7 cells [70]. As a weak basic drug, the uptake, export, and intracellular behavior of DOX in MCF7 cells might furthermore be affected by a change in its propensity of being protonated [71, 72]. The concept of avoiding drug resistance by using various delivery systems was already tested for DOX. Common approaches utilize nanoparticles for delivery [73], such as polyester-based hyperbranched dendritic-linear (HBDL)-based nanoparticles, which could effectively overcome MGST1 mediated drug resistance in MCF7 cells [74] or mesoporous silica nanoparticles (MSNs) that were used to deliver DOX in a xenograft nude mouse model [59]. These and other targeted delivery systems such as homing peptides, immunoliposomes, or other nanoparticle approaches can furthermore be combined with the prodrug concept, thus providing the opportunity to merge an enhanced drug delivery with a selective activation mechanism, which might enable a more optimal targeting of cancer cells [75-77].

Accumulation of prodrugs in the tumor cells through selective targeting would furthermore promote complementary effects that in turn augment the toxicity induced by the freed parental drug. Prodrug activation, for instance, involves the covalent 
addition of GSH to the sulfonamide moiety, which thus may reduce the GSH pool depending on the molar ratio of prodrug-to-GSH within the cell. Importantly, even a minor reduction might already improve cancer cell death in tumors through increased oxidative stress or a reduction of the detoxifying capacity in these cells $[35,78]$. DOX, for instance, has already been shown to benefit significantly from decreased GSH levels, which might thus be part of the mechanism of ANS-DOX and DNS-DOX toxicity[64]. Additionally, prodrugs might target intracellular nucleophiles other than GSH through their electrophilic sulfonamide bond. A prime candidate in this context is the redox regulatory enzyme TrxR1. It is the key enzyme in the antioxidant Trx system, overexpressed in many tumors and easily inhibited by electrophilic compounds due to a reactive and accessible Sec-residue within its active site. In fact, not only is TrxR1 considered to be an attractive anticancer target by itself [31], but inhibition of the enzyme has been suggested to be part of the mechanism of many drugs [32]. Despite a rapid activation via GSH/GST, both DNS-DOX and ANS-DOX could inhibit TrxR1 in vitro and in cell culture with the more reactive DNS-DOX being more efficient than ANSDOX. The ability to target TrxR1 was hereby dependent on the GST levels, as the activated drug is not targeting the enzyme. Nonetheless, the enzyme was inhibited in GSTA1 overexpressing V79 cells, suggesting that disruption of the Trx system is indeed a contributing factor. The mechanism may involve the induction of oxidative stress through a reduction in the antioxidant capacity or the disruption of redox signaling pathways. Furthermore, inhibition of TrxR1 is suggested to effectively synergize with targeting of the GSH system in the killing of cancer cells as both systems serve to back each other up $[33,34]$. Designing prodrugs that concomitantly target the Trx and GSH systems in cancer cells while releasing a drug that benefits from these concomitant effects would thus be a suitable strategy to target cancer cells.

In parallel we included etoposide and an etoposide based prodrug in this study to validate it as a candidate for the development of novel GST activated prodrugs. The structure of the drug was modified such that a nitro acetyl-benzene moiety became conjugated to the etoposide (ANS-etoposide) through a sulfonic acid ester linkage (Fig. 1A). The choice of ANS as the prodrug modification principle rests also on the fact that sulfonic acid ester linkages are more labile and that DNS modified etoposide would thus be less stable. Treating MGST1 overexpressing cells with ANS-etoposide showed an enzyme-dependent increase in toxicity, which, however, was weakly concentration dependent and had a less potent toxic profile compared to etoposide, suggesting either a compromised uptake or a limited activation. GSTA1 overexpression, however, showed a significant resistance against etoposide induced toxicity compared to control cells. Whereas treating the cells with the prodrug ANS-etoposide, the etoposide-dependent resistance was totally abolished, indicating a GSTA1-dependent activation of the prodrug. Potentially, the GSTA1-dependent activation of ANS-etoposide might compensate for the etoposide-induced resistance caused by overexpression. The 
GSTA1-dependent protection to etoposide toxicity could potentially be explained by the intracellular distribution of the enzyme and the mechanism of etoposide induced cell death, which has not been completely delineated yet. Jamil et al. showed, for instance, that low concentrations of etoposide could induce cell death via the mitochondrial arm of p53 [79], a cellular compartment where also GSTA1 was shown to be present [80]. Furthermore, it is not known whether the chemical modification of etoposide to ANSetoposide alters the DNA topoisomerase II inhibitory properties of the molecule (we know that the modification of DOX occurs at an amino group critical for toxic properties). Also, the chemical liability of the sulfonic acid ester bond could potentially lead to a rapid cellular, perhaps enzyme catalyzed, conversion to etoposide thus showing little difference compared to etoposide treatment. This idea needs to be tested with a less reactive mononitro derivative.

Despite the above outlined benefits of GST/GSH activatable drugs, their development and use may have potential drawbacks as they may become highly toxic in sites rich in GSTs such as the liver [81]. To circumvent this problem we propose to use established drugs with known toxicity profiles, particularly those that are known to have low liver toxicity, as the basis for prodrugs. A very rapid activation of ANS-DOX in liver for example, may lead to the same toxicity as if the DOX would have been used directly. However, at the same time toxicity in all other cells, except the GSTA1 overexpressing cancer cells, would be reduced thus showing overall fewer side effects. Our experiments on V79 control cells and GSTA1 overexpressing V79 cells as a model system essentially showed that ANS-DOX was less toxic in the V79 control cells compared to DNS-DOX and DOX, whereas in GSTA1 overexpressing cells toxicity was similar no matter if DOX, ANS-DOX, or DNS-DOX was used (see Fig. 3). Nonetheless, future in vivo studies are needed to fully grasp the potential short-term and long-term side effects of these prodrugs.

This study further explored the concept of using GST activated prodrugs in cancer treatment. We highlight that a low GST catalyzed conversion rate and a low background activity optimizes the GST-dependent accumulated of the activated drug and thus the augmentation of drug induced toxicity. Further, we show that rational modifications of the different DOX derivatives can affect not only the uptake and stability, but also the GST catalyzed conversion rate of this prodrug. We also show that decreased GSH levels and disruption of the Trx system via TrxR1 inhibition are concomitant factors of prodrug toxicity that likely synergize with the effects of the freed parental drug. Furthermore, we expanded the prodrug concept toward etoposide. Tailored modification might thus produce prodrugs that can be used to target specific types of cancers depending on their GST levels and composition as well as those displaying drug resistance $[82,83]$. Designing different prodrug-types for different patient populations could contribute to a more personalized cancer therapy approach, were the therapeutic window is increased. Notably, TLK-286, a previously developed prodrug that targets GSTP1 
overexpressing cancer cells went through to Phase III clinical trials showing the potential of this strategy $[13,14]$.

In conclusion, this study showed that the modification of existing drugs at the common amino or hydroxyl functional groups could be an effective way to develop novel selective drugs, which can overcome resistance mechanisms occurring in conventional treatments. Altering properties of DOX showed promising results to target resistant cancer cells and for the selective targeting of GST overexpressing tumors, thus potentially also reducing the side effects to normal tissue.

\section{Acknowledgments}

These studies were financially supported by the Swedish Research council, the Swedish Cancer Society, SSF, Carl Tryggers Stiftelse, AstraZeneca, and funds from the University of Maastricht and Karolinska Institutet. J.Z. was financially supported by the Swedish Research Council (No. 524-2011-6998). MEXT (Ministery of Education, Culture, Sports, Science and Technology of Japan) and NEDO (New Energy and Industrial Technology Development Organization of Japan) financially supported H.A. 


\section{References}

1. Darzynkiewicz, Z., F. Traganos, and D. Wlodkowic, Impaired DNA damage response--an Achilles' heel sensitizing cancer to chemotherapy and radiotherapy. Eur J Pharmacol, 2009. 625(1-3): p. 143-50.

2. Simunek, T., et al., Anthracycline-induced cardiotoxicity: overview of studies examining the roles of oxidative stress and free cellular iron. Pharmacol Rep, 2009. 61(1): p. 154-71.

3. van Acker, F.A., et al., 7-monohydroxyethylrutoside protects against chronic doxorubicin-induced cardiotoxicity when administered only once per week. Clin Cancer Res, 2000. 6(4): p. 1337-41.

4. Tredan, O., et al., Drug resistance and the solid tumor microenvironment. J Natl Cancer Inst, 2007. 99(19): p. 1441-54.

5. Lippert, T.H., H.J. Ruoff, and M. Volm, Resistance in malignant tumors: can resistance assays optimize cytostatic chemotherapy? Pharmacology, 2008. 81(3): p. 196-203.

6. Rautio, J., et al., Prodrugs: design and clinical applications. Nat Rev Drug Discov, 2008. 7(3): p. 255-70.

7. Johansson, K., et al., Microsomal glutathione transferase 1 in anticancer drug resistance. Carcinogenesis, 2007. 28(2): p. 465-70.

8. Johansson, K., et al., Characterization of new potential anticancer drugs designed to overcome glutathione transferase mediated resistance. Mol Pharm, 2011. 8(5): p. 1698-708.

9. Scotlandi, K., et al., Overcoming resistance to conventional drugs in Ewing sarcoma and identification of molecular predictors of outcome. J Clin Oncol, 2009. 27(13): p. 2209-16.

10. Townsend, D.M. and K.D. Tew, The role of glutathione-S-transferase in anti-cancer drug resistance. Oncogene, 2003. 22(47): p. 7369-75.

11. Sau, A., et al., Glutathione transferases and development of new principles to overcome drug resistance. Arch Biochem Biophys, 2010. 500(2): p. 116-22.

12. Hayes, J.D., J.U. Flanagan, and I.R. Jowsey, Glutathione transferases. Annu Rev Pharmacol Toxicol, 2005. 45: p. 51-88.

13. Tew, K.D. and D.M. Townsend, Redox platforms in cancer drug discovery and development. Curr Opin Chem Biol, 2011. 15(1): p. 156-61.

14. Tew, K.D., TLK-286: a novel glutathione S-transferase-activated prodrug. Expert Opin Investig Drugs, 2005. 14(8): p. 1047-54.

15. Singh, S., Cytoprotective and regulatory functions of glutathione S-transferases in cancer cell proliferation and cell death. Cancer Chemother Pharmacol, 2015. 75(1): p. 1-15.

16. Di Pietro, G., L.A. Magno, and F. Rios-Santos, Glutathione S-transferases: an overview in cancer research. Expert Opin Drug Metab Toxicol, 2010. 6(2): p. 153-70.

17. Mcllwain, C.C., D.M. Townsend, and K.D. Tew, Glutathione S-transferase polymorphisms: cancer incidence and therapy. Oncogene, 2006. 25(11): p. 1639-48.

18. Jakobsson, P.J., et al., Common structural features of MAPEG -- a widespread superfamily of membrane associated proteins with highly divergent functions in eicosanoid and glutathione metabolism. Protein Sci, 1999. 8(3): p. 689-92.

19. Singhal, S.S., et al., Antioxidant role of glutathione S-transferases: 4-Hydroxynonenal, a key molecule in stress-mediated signaling. Toxicol Appl Pharmacol, 2015. 289(3): p. 361-70.

20. Kalinina, E.V., N.N. Chernov, and M.D. Novichkova, Role of glutathione, glutathione transferase, and glutaredoxin in regulation of redox-dependent processes. Biochemistry (Mosc), 2014. 79(13): p. 1562-83.

21. Hengstler, J.G., et al., Resistance factors in colon cancer tissue and the adjacent normal colon tissue: glutathione S-transferases alpha and pi, glutathione and aldehyde dehydrogenase. Cancer Lett, 1998. 128(1): p. 105-12.

22. Oguztuzun, S., et al., The expression of GST isoenzymes and p53 in non-small cell lung cancer. Folia Histochemica Et Cytobiologica, 2010. 48(1): p. 122-127.

23. Carmichael, J., et al., Glutathione S-Transferase Isoenzymes and Glutathione-Peroxidase Activity in Normal and Tumor Samples from Human-Lung. Carcinogenesis, 1988. 9(9): p. 1617-1621. 
24. Oguztuzun, S., et al., Expression of glutathione-S-transferases isoenzymes and p53 in exfoliated human bladder cancer cells. Urologic Oncology-Seminars and Original Investigations, 2011. 29(5): p. 538-544.

25. Chuang, Overexpression of glutathione S-transferase alpha in clear cell renal cell carcinoma. (vol 123, pg 421, 2005). American Journal of Clinical Pathology, 2005. 124(3): p. 476-476.

26. Giaccia, A.J., et al., The Hypersensitivity of the Chinese-Hamster Ovary Variant Bl-10 to Bleomycin Killing Is Due to a Lack of Glutathione S-Transferase-Alpha Activity. Cancer Research, 1991. 51(16): p. 4463-4469.

27. Tew, K.D., Glutathione-associated enzymes in anticancer drug resistance. Cancer Res, 1994. 54(16): p. 4313-20.

28. Sarkar, D., et al., Overexpression of glutathione-S-transferase A1 in benign adrenocortical adenomas from patients with Cushing's syndrome. J Clin Endocrinol Metab, 2001. 86(4): p. 1653-9.

29. Beaumont, P.O., et al., Role of glutathione S-transferases in the resistance of human colon cancer cell lines to doxorubicin. Cancer Res, 1998. 58(5): p. 947-55.

30. Harbottle, A., et al., Role of glutathione S-transferase P1, P-glycoprotein and multidrug resistanceassociated protein 1 in acquired doxorubicin resistance. Int J Cancer, 2001. 92(6): p. 777-83.

31. Urig, S. and K. Becker, On the potential of thioredoxin reductase inhibitors for cancer therapy. Seminars in Cancer Biology, 2006. 16(6): p. 452-465.

32. Cebula, M., E.E. Schmidt, and E.S.J. Arner, TrxR1 as a Potent Regulator of the Nrf2-Keap1 Response System. Antioxidants \& Redox Signaling, 2015. 23(10): p. 823-853.

33. Harris, I.S., et al., Glutathione and Thioredoxin Antioxidant Pathways Synergize to Drive Cancer Initiation and Progression. Cancer Cell, 2015. 27(2): p. 211-222.

34. Fath, M.A., et al., Enhancement of Carboplatin-Mediated Lung Cancer Cell Killing by Simultaneous Disruption of Glutathione and Thioredoxin Metabolism. Clinical Cancer Research, 2011. 17(19): p. 62066217.

35. Trachootham, D., J. Alexandre, and P. Huang, Targeting cancer cells by ROS-mediated mechanisms: a radical therapeutic approach? Nat Rev Drug Discov, 2009. 8(7): p. 579-91.

36. Balamuth, N.J. and R.B. Womer, Ewing's sarcoma. Lancet Oncol, 2010. 11(2): p. 184-92.

37. Habermann, T.M., Antibody therapy in aggressive lymphomas. Hematology Am Soc Hematol Educ Program, 2007: p. 257-64.

38. Horn, L., E.L. Castellanos, and D.H. Johnson, Update on new drugs in small cell lung cancer. Expert Opin Investig Drugs, 2011. 20(4): p. 441-5.

39. Straus, D.J., Chemotherapy only for localized Hodgkin lymphoma. J Intern Med, 2011. 270(3): p. 197-205.

40. Sevim, H., J.F. Parkinson, and K.L. McDonald, Etoposide-mediated glioblastoma cell death: dependent or independent on the expression of its target, topoisomerase I/ alpha? J Cancer Res Clin Oncol, 2011. 137(11): p. 1705-12.

41. Karpinich, N.O., et al., The course of etoposide-induced apoptosis from damage to DNA and p53 activation to mitochondrial release of cytochrome c. J Biol Chem, 2002. 277(19): p. 16547-52.

42. Ratain, M.J., et al., Acute nonlymphocytic leukemia following etoposide and cisplatin combination chemotherapy for advanced non-small-cell carcinoma of the lung. Blood, 1987. 70(5): p. 1412-7.

43. Mistry, A.R., et al., DNA topoisomerase II in therapy-related acute promyelocytic leukemia. N Engl J Med, 2005. 352(15): p. 1529-38.

44. Habig, W.H., M.J. Pabst, and W.B. Jakoby, Glutathione S-Transferases - First Enzymatic Step in Mercapturic Acid Formation. Journal of Biological Chemistry, 1974. 249(22): p. 7130-7139.

45. Sundberg, K., et al., Glutathione conjugation and DNA adduct formation of dibenzo[a,I]pyrene and benzo[a]pyrene diol epoxides in V79 cells stably expressing different human glutathione transferases. Chemical Research in Toxicology, 2002. 15(2): p. 170-179.

46. Mosmann, T., Rapid Colorimetric Assay for Cellular Growth and Survival - Application to Proliferation and Cyto-Toxicity Assays. Journal of Immunological Methods, 1983. 65(1-2): p. 55-63.

47. Sundqvist, K., et al., Cyto-Toxic and Genotoxic Effects of Areca Nut-Related Compounds in Cultured Human Buccal Epithelial-Cells. Cancer Research, 1989. 49(19): p. 5294-5298.

48. Jarvis, I.W.H., et al., Persistent activation of DNA damage signaling in response to complex mixtures of PAHs in air particulate matter. Toxicology and Applied Pharmacology, 2013. 266(3): p. 408-418. 


\section{Chapter 7}

49. Rahman, I., A. Kode, and S.K. Biswas, Assay for quantitative determination of glutathione and glutathione disulfide levels using enzymatic recycling method. Nature Protocols, 2006. 1(6): p. 3159-3165.

50. Ren, X.L., et al., Mutagenesis of Structural Half-Cystine Residues in Human Thioredoxin and Effects on the Regulation of Activity by Selenodiglutathione. Biochemistry, 1993. 32(37): p. 9701-9708.

51. Xu, J.Q. and E.S.J. Arner, Pyrroloquinoline quinone modulates the kinetic parameters of the mammalian selenoprotein thioredoxin reductase 1 and is an inhibitor of glutathione reductase. Biochemical Pharmacology, 2012. 83(6): p. 815-820.

52. Anestal, K., et al., Cell Death by SecTRAPs: Thioredoxin Reductase as a Prooxidant Killer of Cells. Plos One, 2008. 3(4).

53. Cenas, N., et al., Interactions of quinones with thioredoxin reductase - A challenge to the antioxidant role of the mammalian selenoprotein. Journal of Biological Chemistry, 2004. 279(4): p. 2583-2592.

54. Prast-Nielsen, S., et al., Noble metal targeting of thioredoxin reductase - covalent complexes with thioredoxin and thioredoxin-related protein of 14 kDa triggered by cisplatin. Free Radical Biology and Medicine, 2010. 49(11): p. 1765-1778.

55. Arner, E.S. and A. Holmgren, Measurement of thioredoxin and thioredoxin reductase. Curr Protoc Toxicol, 2001. Chapter 7: p. Unit 74.

56. Shibata, A., et al., Fluorogenic probes using 4-substituted-2-nitrobenzenesulfonyl derivatives as caging groups for the analysis of human glutathione transferase catalyzed reactions. Analyst, 2013. 138(24): p. 7326-7330.

57. Morgenstern, R., et al., The Distribution of Microsomal Glutathione Transferase among Different Organelles, Different Organs, and Different Organisms. Biochemical Pharmacology, 1984. 33(22): p. 3609-3614.

58. Lou, P.J., et al., Reversal of doxorubicin resistance in breast cancer cells by photochemical internalization. Int J Cancer, 2006. 119(11): p. 2692-8.

59. Wang, X., et al., Increasing the cytotoxicity of doxorubicin in breast cancer MCF-7 cells with multidrug resistance using a mesoporous silica nanoparticle drug delivery system. Int J Clin Exp Pathol, 2014. 7(4): p. 1337-47.

60. Donmez, Y. and U. Gunduz, Reversal of multidrug resistance by small interfering RNA (siRNA) in doxorubicin-resistant MCF-7 breast cancer cells. Biomed Pharmacother, 2011. 65(2): p. 85-9.

61. AbuHammad, S. and M. Zihlif, Gene expression alterations in doxorubicin resistant MCF7 breast cancer cell line. Genomics, 2013. 101(4): p. 213-20.

62. De, U., et al., A novel anthracene derivative, MHY412, induces apoptosis in doxorubicin-resistant MCF7/Adr human breast cancer cells through cell cycle arrest and downregulation of P-glycoprotein expression. Int J Oncol, 2014. 44(1): p. 167-76.

63. Alpsoy, A., S. Yasa, and U. Gunduz, Etoposide resistance in MCF-7 breast cancer cell line is marked by multiple mechanisms. Biomedicine \& Pharmacotherapy, 2014. 68(3): p. 351-355.

64. Kisara, S., et al., Effect of glutathione depletion by buthionine sulfoximine on doxorubicin toxicity in mice. Res Commun Mol Pathol Pharmacol, 1995. 89(3): p. 401-10.

65. Gromer, S., et al., Human placenta thioredoxin reductase. Isolation of the selenoenzyme, steady state kinetics, and inhibition by therapeutic gold compounds. The Journal of biological chemistry, 1998. 273(32): p. 20096-101.

66. Omata, Y., et al., Sublethal concentrations of diverse gold compounds inhibit mammalian cytosolic thioredoxin reductase (TrXR1). Toxicology in vitro : an international journal published in association with BIBRA, 2006. 20(6): p. 882-90.

67. Holohan, C., et al., Cancer drug resistance: an evolving paradigm. Nature Reviews Cancer, 2013. 13(10): p. 714-726.

68. Nandurdikar, R.S., et al., Structural modifications modulate stability of glutathione-activated arylated diazeniumdiolate prodrugs. Bioorganic \& Medicinal Chemistry, 2012. 20(9): p. 3094-3099.

69. Doyle, L.A., et al., A multidrug resistance transporter from human MCF-7 breast cancer cells. Proceedings of the National Academy of Sciences of the United States of America, 1998. 95(26): p. 15665-15670. 
70. Wind, N.S. and I. Holen, Multidrug resistance in breast cancer: from in vitro models to clinical studies. Int J Breast Cancer, 2011. 2011: p. 967419.

71. Swietach, P., et al., Importance of intracellular pH in determining the uptake and efficacy of the weakly basic chemotherapeutic drug, doxorubicin. PLoS One, 2012. 7(4): p. e35949.

72. Webb, B.A., et al., Dysregulated pH: a perfect storm for cancer progression. Nat Rev Cancer, 2011. 11(9): p. 671-7.

73. Xue, X. and X.J. Liang, Overcoming drug efflux-based multidrug resistance in cancer with nanotechnology. Chinese Journal of Cancer, 2012. 31(2): p. 100-109.

74. Zeng, X.H., R. Morgenstern, and A.M. Nystrom, Nanoparticle-directed sub-cellular localization of doxorubicin and the sensitization breast cancer cells by circumventing GST-Mediated drug resistance. Biomaterials, 2014. 35(4): p. 1227-1239.

75. Safari, J. and Z. Zarnegar, Advanced drug delivery systems: Nanotechnology of health design A review. Journal of Saudi Chemical Society, 2014. 18(2): p. 85-99.

76. Tiwari, G., et al., Drug delivery systems: An updated review. Int J Pharm Investig, 2012. 2(1): p. 2-11.

77. Svensen, N., J.G. Walton, and M. Bradley, Peptides for cell-selective drug delivery. Trends Pharmacol Sci, 2012. 33(4): p. 186-92.

78. Traverso, N., et al., Role of glutathione in cancer progression and chemoresistance. Oxid Med Cell Longev, 2013. 2013: p. 972913.

79. Jamil, S., et al., Etoposide induces cell death via mitochondrial-dependent actions of p53. Cancer Cell International, 2015. 15.

80. Raza, H., et al., Multiple isoforms of mitochondrial glutathione S-transferases and their differential induction under oxidative stress. Biochemical Journal, 2002. 366: p. 45-55.

81. Deponte, M., Glutathione catalysis and the reaction mechanisms of glutathione-dependent enzymes. Biochimica Et Biophysica Acta-General Subjects, 2013. 1830(5): p. 3217-3266.

82. Morgenstern, R., A simple alternate substrate test can help determine the aqueous or bilayer location of binding sites for hydrophobic ligands/substrates on membrane proteins. Chemical Research in Toxicology, 1998. 11(6): p. 703-707.

83. Morgenstern, R., et al., Studies on the Activity and Activation of Rat-Liver Microsomal Glutathione Transferase, in Particular with a Substrate-Analog Series. Journal of Biological Chemistry, 1988. 263(14): p. 6671-6675. 

Chapter

General discussion 

The success of cancer treatment is dependent amongst others on the resistance to the treatment and therapy tolerability of healthy tissues. Treatment resistance can be acquired during oncogenic/tumor formation, but can also arise during the treatment course. A tolerable treatment depends on the (over)sensitivity of normal tissues. Currently there is an urgent need to reduce both treatment resistance and normal tissue toxicity. The discovery of treatment-related biomarkers predicting outcome is of great interest in cancer therapy. A biomarker might help patients and clinicians to select the appropriate cancer treatment leading to a personalized cancer treatment approach. In this thesis, it was investigated how intrinsic or extrinsic changes of the tumor can contribute to a personalized patient treatment plan. The role of mitochondrial function, ROS and hypoxia modulation in tumors were investigated in view of the possibility of implementing them in a personalized treatment approach. To assess this, mitochondrial dysfunction has been acquired using different approaches such as mtDNA depletion (chapter 3 and 4), cytoplasmic hybrids (chapter 4), OXPHOS complex I inhibitors (chapter 4) and complex I inhibition in a dual-targeting approach (chapter 5). Additionally, repurposing drug (chapter 6) and prodrug approaches (chapter 7) have been evaluated. Molecular approaches have been used to investigate the effect of energetic stress and/or stress inducers (radiation and hypoxia) in relation to mitochondrial function (chapter 3 and 4). Different drug strategies (dual-targeting CAIXphenformin, repurposing nitroglycerin, prodrugs such as ANS-DOX) were investigated for the modulation of cancer treatment limiting parameters such as toxicity, hypoxia and drug resistance (chapter 5, 6 and 7). This chapter discusses the studies described in this thesis in relation to current literature and addresses also future perspectives.

\section{The influence of mitochondrial DNA and mitochondrial dysfunction on cancer therapy.}

During evolution mitochondria kept at least a part of their own DNA which consists out of 13 genes encoding for different subunits of the oxidative phosphorylation [1, 2]. Where mitochondria are often referred to as the 'power plant of the cell' they are important for several other cellular systems. They play an important role in calcium homeostasis, apoptosis, reactive oxygen species signaling and production, redox reactions and they contribute to the maintenance of the pyrimidines pool [3-7]. The human mitochondrial genome is a very dynamic system. It is almost completely coding and multiple copies of mtDNA occur within every mitochondrion.

Multiple variations of mtDNA, like point mutations, deletions and copy number alterations have been reported in patients' populations. Also in cancer patients mtDNA alterations have been observed (chapter 2). Both somatic and germline mutations were found to relate to cancer, either to cancer risk or in association with tumor progression 
[8-12]. Petros et al. have demonstrated in a cohort study for prostate cancer an association with tumor progression for different germline mtDNA mutations in cytochrome oxidase. In a proof-of-principle study they showed for a germline mutation in ATP6, which codes for a subunit of ATP synthase (Complex $V$ of the OXPHOS), a 7-fold larger tumor volume for mutants compared to wild-type tumors [13]. However, there are also studies that indicate that most mtDNA variations could potentially just be passenger mutations. These passenger mutations are mutations that have no influence on the cellular characteristics and don't drive tumorigenesis and tumor progression [14, 15]. Additionally, variations in mtDNA copy numbers levels have been reported to be associated with an increased risk for tumorigenesis in breast cancer [16], RCCs [17] and non-small cell lung [18] cancer patients but contradictory results also have been reported [19-21]. A recent meta-analysis observed an association in breast cancer and lymphomas with increased mtDNA copy numbers [22]. In contrast an increased mtDNA copy number was found to be negatively associated with hepatic malignancies [22].

Currently in cancer treatment, an increasing number of drugs are being developed or used which interact with the mitochondrial function and/or the mtDNA. For instance, chemotherapeutics can induce a reduction in mtDNA copy number levels $[23,24]$. A conventional chemotherapeutic drug interacting with mitochondrial function is cisplatin, since it binds to mtDNA and impairs mtDNA synthesis [24, 25].

mtDNA depletion is often an extreme method to investigate mtDNA related mitochondrial dysfunction, in both tumorigenic or normal tissue. In chapter 3 we used such mtDNA depleted models to study the effect of mitochondrial dysfunction on irradiation outcome. Several research groups observed different radiation responses in mtDNA models, from a radioresistant phenotype in various (tumor) cell lines in vitro $[26,27]$ to even an increased radiation response in an in vivo model [28]. In our study, also distinctive radiation responses were found. Cells depleted from their mitochondrial DNA displayed a more radiation resistant phenotype for an immortalized bronchial epithelial cell line while a more radiosensitive phenotype for two different tumor cell lines, although intrinsic radiosensitivity (surviving fraction after 2Gy) was not altered. Also for other treatment modalities, cells depleted from their mtDNA can show a more resistant phenotype, as for example has been observed for cisplatin exposure [25]. This mitochondrial dysfunction dependent cisplatin resistance might be dependent on glutathione levels [24]. In our study we observed that the antioxidant capacity of cells, i.e. altered GSH:GSSG ratio's and mitochondrial super oxide dismutase (SOD2), could possibly explain a reduced response upon irradiation. Similar results have been reported by others $[29,30]$. Additionally, our findings on clonogenic survival were in agreement with the observed increased residual DNA damage upon mtDNA depletion.

DNA damage repair upon irradiation often has been suggested to be dependent on ATP [31-33]. Additionally, it has been shown that oxygen consumption and mitochondrial 
ATP generation in irradiated normal cells was concurrent with the mitochondrial relocation of the cell-cycle dependent kinase CDK1 which could be inhibited by CDK1 phosphorylation-deficient $\mathrm{Cl}$ subunits [34]. Also for tumor cells it has been found that mitochondrial derived ATP is enhanced upon irradiation leading to increased survival [35]. Based on these reports, we investigated if we could observe any differences in ATP levels upon irradiation. However, for the mtDNA depleted cell lines investigated we did not observe any alterations in cellular ATP levels in agreement with Lu et al. [35]. This can be explained by the fact that tumor cells are very flexible in altering their metabolism and have the ability to acquire necessary nutrients from their environment and utilize them in order to maintain viability and generate new biomass (for an excellent recent review see [36]).

\section{Metabolic transformation of cancer}

Metabolic adaption and transformation of cells is a phenomenon often observed in cancer. We observed in chapter 3 a decrease of OXPHOS upon normoxic conditions and an increased glucose metabolism for the mtDNA depleted cell lines but no changes in ATP production. Tumor cells require large amounts of energy in order to stimulate their growth. Tumors hijack multiple systems in order to obtain enough energy for expansion. Multiple metabolic pathways are found to be deregulated such as glutamine, amino acid and lipid metabolism, the pentose phosphate pathway as well as glycolysis [36-43]. The aerobic glycolysis of tumors, already observed by Warburg [41] in the beginning of the $20^{\text {th }}$ century, is currently exploited in the clinic using FDG-PET imaging to detect tumors and to monitor tumor progression [44]. However, recently the validity of the Warburg effect has been questioned. Although it is often suggested that in tumors the oxidative phosphorylation (OXPHOS) is absent due to the upregulation of glycolysis, like a crabtree effect, both metabolic profiles often co-exist in well-oxygenated tumor areas in order to meet the metabolic demands of the tumor. Observed changes in OXPHOS are not only heterogeneous between tissue types but also within samples obtained from the same tumor, suggesting that differences are not only caused by the environment of different tumor types but also are subject to physiological conditions such as hypoxia or genetic alterations [45].

\section{CAIX and OXPHOS inhibition: how do they interact?}

It has been suggesting that in fastly proliferating tumor cells glucose metabolism is dominant since CDC42 expression (a gene involved in cell division) was found to be correlated with the expression of the glycolysis pathway [45]. In areas with reduced oxygen, the hypoxic areas, hypoxia inducible factor 1 alpha (HIF-1 $\alpha$ ) downregulates 
OXPHOS [46] and promotes glycolysis via regulation of expression of the glucose transporters GLUT1 and GLUT3 and the lactate efflux transporter MCT4 [11, 47, 48]. Downregulation of OXPHOS gene expression was found to correlate with a worse prognosis in different patients populations e.g. bladder urothelial carcinoma, cervical squamous cell carcinoma, endocervical adenocarcinoma, glioblastoma multiforme etc. [49]. OXPHOS downregulation also was found to correlate with the induction of the epithelial-to-mesenchymal transition which is linked to invasion and metastasis formation [49-51]. HIF-1 $\alpha$ stabilization can also occur under normoxic conditions. Succinate, which accumulates upon succinate dehydrogenase inhibition, causes reduction of the prolyl hydroxylases resulting in stabilization of HIF-1 $\alpha$ under normoxic conditions, similar as has been described for VHL mutations [52]. The discovery of mutations in nuclear TCA genes such as succinate dehydrogenase [53, 54], fumarate hydratase [55, 56] and iso-citrate dehydrogenases [57-59] suggests a role for these mutations in oncogenic transformation and accelerated the interest in mitochondrial encoded genes.

In chapter 4 we investigated if the increase of carbonic anhydrase IX (CAIX) could be observed in tumor cells displaying a mitochondrial dysfunction. The hypoxia regulated CAIX plays an important role in sustaining the cellular pH balance [60,61]. Here we remarkably didn't observe an increase in CAIX expression under both normoxic and hypoxic conditions for mtDNA depleted tumor cell lines (from chapter 3 ) as well as for a newly generated osteosarcoma model harboring a mtDNA m.3243A>G point mutation, i.e. a cybrid model (chapter 4). This mutation is responsible for the "mitochondrial encephalomyopathy, lactic acidosis and stroke-like episodes" (MELAS) syndrome $[62,63]$. In contrast, we observed a decreased CAIX expression under hypoxic conditions in these models, which seemed to be dependent on the mutation percentage of the mtDNA. As one cell can contain up to thousands of copies of mtDNA, both mutated and wild-type mtDNA can co-exist ranging from 1 to $99 \%$ within the same mitochondrion, cell, tissue or organism making heteroplasmy levels another important factor to take in account. When a mutation is present in all copies of the mtDNA, this is referred to as homoplasmy. From the research field of mitochondrial diseases, we have learned that the mutation often should exceed a certain threshold in order for a patient to show pathological symptoms in case of severe inherited mitochondrial diseases (chapter 2). These thresholds can be mutation specific [64] as well as tissue specific [65]. Homoplasmic mtDNA variations also can be involved in less severe diseases (e.g. hearing lost) which need an additional trigger (exposure to the antibiotic aminoglycoside) to demonstrate their pathogenic phenotype $[66,67]$. We expect a similar mechanism in case of treatment-related pathologies such as radiation-induced toxicity.

Increased lactic acid levels are often observed in mitochondrial diseases and tumorigenic tissues $[63,68]$. In tumors this increased lactate production is the result of 
both the Warburg (aerobic glycolysis) and the Pasteur effect (anaerobic glycolysis) and has been found to be correlated with radiation resistance in a xenograft model and related to a worse prognosis in patients $[69,70]$. Overall, enhanced glycolysis within tumors will result in an increased acidification of the intracellular $\mathrm{pH}$. In order to maintain a physiological intracellular $\mathrm{pH}$, this acidification will be counteracted by an increased carbonic anhydrase activity. Tumor cells under metabolic stress and hypoxia adjust their metabolism and cell signaling in order to increase their survival. In chapter 4 we exposed cells to both metabolic stress and limited oxygen tensions. When cells suffer from both metabolic stress and hypoxia probably other mechanisms are altered possibly explaining the decreased HIF-1 $\alpha$ stabilization and reduced levels of CAIX. One of these mechanism could be the AMPK signaling pathway as it is found to be involved in both mechanisms [71]. Deregulation of AMPK could contribute to an altered translation via inhibition of the mTOR pathway, leading to reduced amounts of HIF-1 $\alpha$ protein and consequently result in decreased CAIX expression levels. Also, other mechanisms such as autophagy could potentially play a role as this process might already be upregulated to meet the cellular metabolic demands accompanied with a decreased need of HIF-1 $\alpha$ signaling. Upregulation of autophagy was observed in cybrids containing a mitochondrial ND5 mutation [72] and is also observed under hypoxic conditions [73]. Mitochondria undergo also autophagy, named mitophagy [74], which can be induced by hypoxia. Mitochondrial NAD-dependent protein deacetylase sirtuin-3 (SIRT3) is a protein with many interesting properties and is found to play a role in inducing fatty acid oxidation [75], AMPK activation [76, 77] and antioxidants as it is found to regulate SOD2 upon calorie restriction mechanisms [78], and is involved in the activation of hypoxia induced mitophagy [79]. Under hypoxic conditions SIRT3 is also involved in destabilization of HIF-1 $\alpha$ under hypoxic conditions [80]. Based on our findings we can conclude that tumor cells exposed to a combination of metabolic stress and hypoxia probably adjust their metabolism and cell signaling in order to survive.

\section{Modulating the $\mathrm{pH}$ balance by using an OXPHOS modulating dual- targeting drug approach}

Combining the important role of CAIX in maintaining the cellular $\mathrm{pH}$ balance and the predominant expression on hypoxic tumor cells correlating with a worse prognosis [81] makes it an appealing target for cancer treatment. In the past, several modalities have been developed to inhibit CAIX function in order to increase the treatment response $[82,83]$. CAIX inhibitors could contribute to intracellular acidification and therefore induce cell death $[84,85]$. A dual-targeting approach where homing strategy is combined with a therapeutic strategy could be a valuable tool to improve success rates of treatment while reducing normal tissue toxicity. Phenformin is a potential drug 
candidate for modification in such a dual-targeting approach and its functionalized form has been investigated in chapter 5 . Phenformin a is very potent drug developed for diabetes mellitus treatment, but was found to induce lactic acidosis in patients and was therefore subsequently withdrawn from the market. Currently, a closely related drug, metformin is still used in daily practice as the incidence rates of lactic acidosis are lower. Metformin displays improved treatment response rates in cancer patients, while studies in cancer patients for phenformin have yet to be performed, although preclinical studies are promising $[86,87]$. The proposed mechanism of action of phenformin is its ability to inhibit mitochondrial respiration via complex I inhibition and activation of AMPK $[88,89]$. As it reduces oxygen consumption it additionally will improve tumor oxygenation [90]. Targeting phenformin directly to the hypoxic areas of tumor is a promising approach as therapeutic doses and possible adverse effects could be reduced. Unfortunately, we were not able to observe decreased oxygen consumption in tumor cells with increased CAIX expression levels. Our dual-targeting approach using phenformin failed to exhibit its complex I inhibiting properties. However, this doesn't imply that a dual-targeting approach for phenformin or in general is not attractive, but further research is needed focused on making better suited compounds. Although methods to reduce tumor hypoxia can vary a lot in mechanisms, hypoxia modulation remains very important to improve treatment efficiency.

\section{Improvement of tumor oxygenation by a repurposing drug approach}

Neoangiogenesis maintains the blood flow in a tumor but as the tumor mass expands and the demand for oxygen and nutrients increases, tumors become hypoxic as these newly formed vessels are leaky and immature. One approach to counteract hypoxia in tumors would be the reduction of oxygen consumption in tumor cells and thereby reducing the limited diffusion of oxygen in a tumor. Treatments already used for other diseases could attribute to a well-tolerated and cheap therapy to alter the oxygen status of a tumor. Repurposing drugs like metformin, a drug developed for diabetes mellitus type 2, could lead to reduced costs in drug development and is currently investigated in clinical trials (see above). Metformin is just like phenformin a biguanide however it is less potent probably due to a lower lipophilicity and therefore uptake in cells and binding to the mitochondrial membrane is decreased [91, 92]. Although having a decreased potency, metformin treated patients harbor a lower incidence of lactic acidosis, making it currently more suitable to use in clinical practice. Recently, derivatives of metformin, mitometformin, were developed in order to target directly the mitochondria and increase the efficiency of the drug [93]. Another approach to reduce tumor hypoxia would be to mature vessel formation, and thereby improve the vasculature of a tumor. However, studies with an antibody against VEGF (bevacizumab) resulted in increased malignancy and metastatic cancers [94]. Next to these findings bevacizumab has also been linked to drug resistance, which has been described to be 
associated with hypoxia-induced autophagy, evidenced by an increased BNIP3 expression [95].

Potentially the increase of the tumor's perfusion would be an alternative approach. Nitroglycerin is a nitric oxide (NO) donor and known for its vasodilation properties in angina pectoris [96]. Yasuda et al. performed a randomized phase II clinical trial in which cisplatin-vinorelbin chemotherapy was combined with nitroglycerin. An improved overall response rate and overall survival was observed in favor of patients treated with nitroglycerin, while treatment toxicity remained equal between the patient groups either receiving a nitroglycerin patch or placebo [97]. However, others didn't observe additional benefit of applying nitroglycerin as adjuvant to standard cancer treatment $[98,99]$. Therefore, we can conclude that the addition of nitroglycerin to standard therapy does not have a major impact on the survival of unselected patients with nonsmall cell lung cancer (NSCLC). In chapter 6 we investigated in a clinical window-ofopportunity trial the influence of the application of nitroglycerin in NSCLC patients on hypoxic tumors. Imaging hypoxia and perfusion was done before and after administration of nitroglycerin. We observed a decrease in the hypoxic fraction for patients with hypoxic tumors and tracer uptake was negatively correlated with tumor perfusion in these hypoxic tumors. Using in vitro experiments we showed that the metabolism of 2-nitroimidazoles is not influenced by nitroglycerin itself and that nitroglycerin does not inhibit mitochondrial respiration, as previously has been suggested [100-102] using human achievable concentrations [103]. Our data suggest that stratification of patients with hypoxic tumors for the use of nitroglycerin patch as adjuvant to anti-cancer treatment in this selected population could be beneficial for their treatment. However, repeated imaging acquisitions could be a burden for patients and therefore further research should be focused on investigating how this inconvenience could be reduced. Possibly a radiogenomics and/or blood biomarkers approach could be helpful in reducing the amount of scans and imaging modalities required.

Hypoxia activated prodrugs (HAP) are another approach to reduce normal tissue side effects and target the hypoxic areas of a tumor at the same time. The first well-known hypoxia activated prodrug is tirapazamine. Phase III trials however didn't show superior effects compared to standard of care, potentially due to the lack of patient selection [104]. PR-104A is another prodrug that was developed to be hypoxia activated and to generate a by-stander effect [105]. Unfortunately, activation of the prodrug PR-104A took also place in some human cell lines under aerobic conditions, suggesting the presence of an aerobic reductase able to activate the prodrug. Indeed aldo-keto reductase $1 \mathrm{C} 3$ could cause the fragmentation of PR-104 upon normoxic conditions [105, 106]. TH-302 is a HAP being developed to directly target the hypoxic areas of tumor and thereby reducing the hypoxic fraction of a tumor and increasing the sensitivity to other treatment options like chemotherapeutic agents and radiotherapy while showing a 
better normal toxicity profile, good preclinical [107, 108] and clinical applications [109]. In two phase III clinical trials, TH-302 failed to show improved outcomes again related to the lack of patient selection in these trials.

\section{The use of prodrugs to overcome chemoresistance}

In hypoxic tumors not only resistance to radiotherapy is often encountered as a problem for effective treatment, also for chemotherapeutics hypoxic tumors often have decreased response rates as many chemotherapeutics require oxygen for optimal response rates [110]. It has been suggested that upon hypoxia, alkylating agents have reduced efficiency due to the increased production of glutathione, which may compete with the target DNA for alkylation [111]. Drug efficiency may further be limited by lack of activation or inactivation, poor drug influx or excessive efflux, alterations in expression levels of the drug target, or the activation or adaptation of pro-survival pathways [112]. Mechanisms involved in the glutathione metabolism play an important role in chemoresistance, but not only in hypoxic tumors. Previously it has been reported that the development of prodrugs could decrease this resistant phenotype and reduce side effects of drugs.

Glutathione S-transferases (GST) is a protein family of significant importance in chemotherapeutic resistance as overexpression is reported in various tumor types [113]. As GSTs serve Phase II detoxification enzymes and are involved in the regulation of oxidative stress, using them to activate the prodrugs could be a valuable concept in overcoming GST mediated drug resistance. Previously it was shown that 2 different prodrug derivatives could be activated by different types of GSTs [114]. In chapter 7 a prodrug was developed to reduce chemotherapeutic resistance of doxorubicin mediated by GSTA1. Indeed, our newly developed GST activated prodrug was able to overcome GSTA1 induced chemoresistance. Our prodrug involves the covalent addition of glutathione (GSH) to the sulfonamide moiety, which potentially reduces the GSH pool within the cell and causes an augmented effect of released parental drug. Already a slight decline in GSH reduces the detoxification capabilities of the cells and would improve oxidative stress mediated cytotoxicity $[115,116]$. The same mechanism might be observed in the response upon radiotherapy in chapter 3, as we found that glutathione levels did potentially rescue the clonogenic survival upon radiation exposure. Next to GSTs, our developed prodrugs inhibit thioredoxin reductase 1 (TrxR1), another important antioxidant enzyme in the thioredoxin (Trx) system. TrxR1 itself has been suggested to be an interesting target for adjuvant cancer therapy [117]. As both the Trx and GSH system can serve as each other's back-up system, designing prodrugs that target both systems at the same time while releasing a cytotoxic compound is a potentially interesting strategy to treat tumor cells. Our strategy for designing these 
GSTs activated pro-drugs was also applied on etoposide. GSTA1 overexpression showed resistance against etoposide; by exposing GSTA1 cells to the prodrug this resistant phenotype was eliminated. It should be mentioned that GSTs are not only highly expressed in tumors but also in the liver [118]. Therefore, using only well-known cytostatic drugs with known toxicity profiles should be used for this approach. The GST mediated release for the prodrug would then be the same compared to the parental drug, but with limited toxicity in cells which don't show GST overexpression.

In the different studies discussed within this chapter we found that modulation of mitochondrial function and mitochondrial proteins can contribute to alterations of treatment responses. Mitochondrial proteins are involved in many complex mechanisms in cancer. Tumor tissues are very heterogeneous and adaptive towards their environment. Heterogeneity is not only observed within a tumor but also between tumors. Elucidating the exact functional consequences of changes in expression, abundance or regulatory capacity of mitochondrial proteins is challenging and makes the search for the perfect modulations for all tumors very arduous. However, stratification of patients will have added value to answer this challenge and identify certain subpopulations which can benefit from these treatment modulations (Fig 1.).

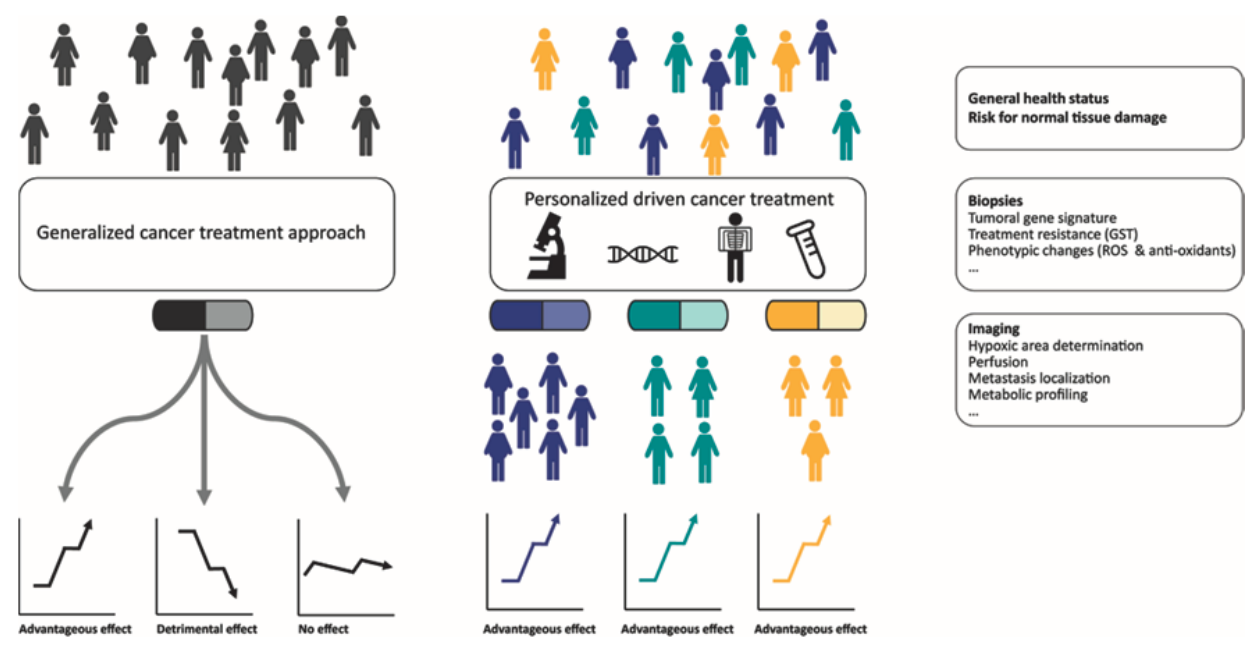

Figure 1. A schematic representation on the contribution of patient stratification on improving response rates in cancer patients. Adapted from [119].

\section{Future perspectives: therapeutic considerations and approaches}

More and more cancer treatment plans are tailor made since therapy integrates the genotype and phenotype of both cancerous and healthy tissue in a personal treatment 
plan for the patient. Prodrugs are developed to be metabolized and exert their pharmacological ability in a specific region of interest. Investigating their specificity of only being metabolized in these regions is an important aspect of generating a safe new prodrug. Activation of the prodrug by the normal tissue should be limited but preferably even be absent. Different potential concepts can be used from nanomolecules (like Doxil (liposomal doxorubicin) [120]) to macromolecules with even the conjugation of an antibody to a cytotoxic agent [121]. This generates a wide range of applications for more specific drugs.

Modulating drugs in order to add specific organelle targeting properties would be an interesting approach. The field of mitochondria and their role in cancer treatment response is currently gaining more and more attention from researchers. Currently drugs which specifically target the mitochondrial function are under investigation and form the future treatment options. Drugs interact with mitochondria in mainly three different manners. 1) Influencing ROS production and antioxidant capacity of the cells. Mitochondria are responsible for a large amount of ROS in cells, recently it has been shown in preclinical studies that an antioxidant preferentially targeting the mitochondria, namely mitoQ, could reduce those effects [122]. For instance, cisplatin causes in approximately 30\% of the patients' nephrotoxicity related to increased ROS production. 2) Inhibition of metabolism. A drug analogue developed to target the mitochondria even more specifically than its parental counterpart is mito-metformin. It was shown to be more potent in inhibiting $\mathrm{Cl}$, activating AMPK, enhancing radiosensitivity in pancreatic tumor cells and abrogating tumor growth in xenografts [93]. 3) Influencing drug metabolism and detoxification. A mitochondrial targeting form of doxorubicin was developed especially to target cells resistant to doxorubicin caused by the ABCB1/P-glycoprotein, a drug efflux protein. In both in vitro and in vivo proliferation and tumor growth was reduced while apoptosis was increased [123].

However, investigating new drugs even if they are modulated and bringing them into the clinic is expensive and timely. The application of new window-of-opportunity trials $[124,125]$ would help to identify promising drugs faster and the beneficiary patient population quicker. Also, repurposing drugs would benefit the patient and reduce costs of drug development. As already exploited in this thesis for nitroglycerin, such studies could also be performed for metformin or phenformin. As both of them already have a well-known toxicity profile applying these drugs in a window-of-opportunity trial would be a valuable new approach to see if they hold promise as cancer treatment for a particular subset of patients. Investigating which subsets of patients could benefit from newly developed drugs would contributes to a personalized cancer treatment approach and hopefully improve the prognosis of cancer patients (Fig 1.). 


\section{References}

1. Gross, J. and D. Bhattacharya, Mitochondrial and plastid evolution in eukaryotes: an outsiders' perspective. Nature reviews. Genetics, 2009. 10(7): p. 495-505.

2. Embley, T.M. and R.P. Hirt, Early branching eukaryotes? (0959-437X (Print)).

3. Redza-Dutordoir, M. and D.A. Averill-Bates, Activation of apoptosis signalling pathways by reactive oxygen species. Biochimica et biophysica acta, 2016. 1863(12): p. 2977-2992.

4. Murgia, M., et al., Controlling metabolism and cell death: at the heart of mitochondrial calcium signalling. Journal of molecular and cellular cardiology, 2009. 46(6): p. 781-8.

5. Chandel, N.S., et al., Reactive oxygen species generated at mitochondrial complex III stabilize hypoxiainducible factor-1alpha during hypoxia: a mechanism of $\mathrm{O} 2$ sensing. The Journal of biological chemistry, 2000. 275(33): p. 25130-8.

6. Mailloux, R.J., X. Jin, and W.G. Willmore, Redox regulation of mitochondrial function with emphasis on cysteine oxidation reactions. Redox biology, 2014. 2: p. 123-39.

7. Khutornenko, A.A., et al., Pyrimidine biosynthesis links mitochondrial respiration to the p53 pathway. Proceedings of the National Academy of Sciences of the United States of America, 2010. 107(29): p. 12828-33.

8. Booker, L.M., et al., North American white mitochondrial haplogroups in prostate and renal cancer. The Journal of urology, 2006. 175(2): p. 468-72; discussion 472-3.

9. Canter, J.A., A.R. Kallianpur, and J.H. Fowke, Re: North American white mitochondrial haplogroups in prostate and renal cancer. The Journal of urology, 2006. 176(5): p. 2308-9; author reply 2309.

10. Bai, R.K., et al., Mitochondrial genetic background modifies breast cancer risk. Cancer research, 2007. 67(10): p. 4687-94.

11. Hayashi, M., et al., Induction of glucose transporter 1 expression through hypoxia-inducible factor 1alpha under hypoxic conditions in trophoblast-derived cells. The Journal of endocrinology, 2004. 183(1): p. 145-54.

12. Stewart, J.B., et al., Simultaneous DNA and RNA Mapping of Somatic Mitochondrial Mutations across Diverse Human Cancers. PLoS genetics, 2015. 11(6): p. e1005333.

13. Petros, J.A., et al., mtDNA mutations increase tumorigenicity in prostate cancer. Proceedings of the National Academy of Sciences of the United States of America, 2005. 102(3): p. 719-24.

14. Calabrese, F.M., et al., A comprehensive characterization of rare mitochondrial DNA variants in neuroblastoma. Oncotarget, 2016. 7(31): p. 49246-49258.

15. Ju, Y.S., et al., Origins and functional consequences of somatic mitochondrial DNA mutations in human cancer. eLife, 2014. 3.

16. Shen, J., et al., Mitochondrial copy number and risk of breast cancer: a pilot study. Mitochondrion, 2010. 10(1): p. 62-8.

17. Hofmann, J.N., et al., A nested case-control study of leukocyte mitochondrial DNA copy number and renal cell carcinoma in the Prostate, Lung, Colorectal and Ovarian Cancer Screening Trial. Carcinogenesis, 2014. 35(5): p. 1028-31.

18. Hosgood, H.D., 3rd, et al., Mitochondrial DNA copy number and lung cancer risk in a prospective cohort study. Carcinogenesis, 2010. 31(5): p. 847-9.

19. Mambo, E., et al., Tumor-specific changes in mtDNA content in human cancer. International journal of cancer, 2005. 116(6): p. 920-4.

20. Dai, J.G., et al., Mitochondrial genome microsatellite instability and copy number alteration in lung carcinomas. Asian Pacific journal of cancer prevention : APJCP, 2013. 14(4): p. 2393-9.

21. Meierhofer, D., et al., Decrease of mitochondrial DNA content and energy metabolism in renal cell carcinoma. Carcinogenesis, 2004. 25(6): p. 1005-10.

22. Hu, L., X. Yao, and Y. Shen, Altered mitochondrial DNA copy number contributes to human cancer risk: evidence from an updated meta-analysis. Sci Rep, 2016. 6: p. 35859. 
23. Ashley, N. and J. Poulton, Mitochondrial DNA is a direct target of anti-cancer anthracycline drugs. Biochemical and biophysical research communications, 2009. 378(3): p. 450-5.

24. Garrido, N., et al., Cisplatin-mediated impairment of mitochondrial DNA metabolism inversely correlates with glutathione levels. Biochem J, 2008. 414(1): p. 93-102.

25. Yang, Z., et al., Cisplatin preferentially binds mitochondrial DNA and voltage-dependent anion channel protein in the mitochondrial membrane of head and neck squamous cell carcinoma: possible role in apoptosis. Clin Cancer Res, 2006. 12(19): p. 5817-25.

26. Cloos, C.R., et al., Mitochondrial DNA depletion induces radioresistance by suppressing G2 checkpoint activation in human pancreatic cancer cells. Radiation research, 2009. 171(5): p. 581-7.

27. Tang, J.T., et al., Mitochondrial DNA influences radiation sensitivity and induction of apoptosis in human fibroblasts. Anticancer research, 1999. 19(6B): p. 4959-64.

28. Bol, V., et al., Reprogramming of tumor metabolism by targeting mitochondria improves tumor response to irradiation. Acta oncologica, 2015. 54(2): p. 266-74.

29. Hosoki, A., et al., Mitochondria-targeted superoxide dismutase (SOD2) regulates radiation resistance and radiation stress response in HeLa cells. Journal of radiation research, 2012. 53(1): p. 58-71.

30. Epperly, M.W., et al., Manganese superoxide dismutase (SOD2) inhibits radiation-induced apoptosis by stabilization of the mitochondrial membrane. Radiation research, 2002. 157(5): p. 568-77.

31. Petermann, E., M. Ziegler, and S.L. Oei, ATP-dependent selection between single nucleotide and long patch base excision repair. DNA repair, 2003. 2(10): p. 1101-14.

32. Lammens, K., et al., The Mre11:Rad50 structure shows an ATP-dependent molecular clamp in DNA double-strand break repair. Cell, 2011. 145(1): p. 54-66.

33. Liu, J., et al., Enhanced cytotoxic effect of low doses of metformin combined with ionizing radiation on hepatoma cells via ATP deprivation and inhibition of DNA repair. Oncology reports, 2012. 28(4): p. 1406-12.

34. Qin, L., et al., CDK1 Enhances Mitochondrial Bioenergetics for Radiation-Induced DNA Repair. Cell reports, 2015. 13(10): p. 2056-63.

35. Lu, C.L., et al., Tumor cells switch to mitochondrial oxidative phosphorylation under radiation via mTORmediated hexokinase II inhibition--a Warburg-reversing effect. PloS one, 2015. 10(3): p. e0121046.

36. Pavlova, N.N. and C.B. Thompson, The Emerging Hallmarks of Cancer Metabolism. Cell metabolism, 2016. 23(1): p. 27-47.

37. Yuneva, M.O., et al., The metabolic profile of tumors depends on both the responsible genetic lesion and tissue type. Cell metabolism, 2012. 15(2): p. 157-70.

38. Yanagida, O., et al., Human L-type amino acid transporter 1 (LAT1): characterization of function and expression in tumor cell lines. Biochimica et biophysica acta, 2001. 1514(2): p. 291-302.

39. De Preter, G., et al., Inhibition of the pentose phosphate pathway by dichloroacetate unravels a missing link between aerobic glycolysis and cancer cell proliferation. Oncotarget, 2016. 7(3): p. 2910-20.

40. Kamphorst, J.J., et al., Hypoxic and Ras-transformed cells support growth by scavenging unsaturated fatty acids from lysophospholipids. Proceedings of the National Academy of Sciences of the United States of America, 2013. 110(22): p. 8882-7.

41. Warburg, O., F. Wind, and E. Negelein, The Metabolism of Tumors in the Body. The Journal of general physiology, 1927. 8(6): p. 519-30.

42. Porporato, P.E., et al., Metabolic changes associated with tumor metastasis, part 2: Mitochondria, lipid and amino acid metabolism. Cellular and molecular life sciences: CMLS, 2016. 73(7): p. 1349-63.

43. Payen, V.L., et al., Metabolic changes associated with tumor metastasis, part 1: tumor pH, glycolysis and the pentose phosphate pathway. Cellular and molecular life sciences: CMLS, 2016. 73(7): p. 1333-48.

44. Zegers, C.M., et al., In vivo quantification of hypoxic and metabolic status of NSCLC tumors using [18F]HX4 and [18F]FDG-PET/CT imaging. Clinical cancer research : an official journal of the American Association for Cancer Research, 2014. 20(24): p. 6389-97.

45. $\mathrm{Hu}$, J., et al., Heterogeneity of tumor-induced gene expression changes in the human metabolic network. Nature biotechnology, 2013. 31(6): p. 522-9.

46. Papandreou, I., et al., HIF-1 mediates adaptation to hypoxia by actively downregulating mitochondrial oxygen consumption. Cell metabolism, 2006. 3(3): p. 187-97. 
47. Ullah, M.S., A.J. Davies, and A.P. Halestrap, The plasma membrane lactate transporter MCT4, but not MCT1, is up-regulated by hypoxia through a HIF-1alpha-dependent mechanism. The Journal of biological chemistry, 2006. 281(14): p. 9030-7.

48. Mimura, I., et al., Dynamic change of chromatin conformation in response to hypoxia enhances the expression of GLUT3 (SLC2A3) by cooperative interaction of hypoxia-inducible factor 1 and KDM3A. Molecular and cellular biology, 2012. 32(15): p. 3018-32.

49. Gaude, E. and C. Frezza, Tissue-specific and convergent metabolic transformation of cancer correlates with metastatic potential and patient survival. Nature communications, 2016. 7: p. 13041.

50. Porporato, P.E., et al., A mitochondrial switch promotes tumor metastasis. Cell reports, 2014. 8(3): p. 754-66.

51. Christiansen, J.J. and A.K. Rajasekaran, Reassessing epithelial to mesenchymal transition as a prerequisite for carcinoma invasion and metastasis. Cancer research, 2006. 66(17): p. 8319-26.

52. Selak, M.A., et al., Succinate links TCA cycle dysfunction to oncogenesis by inhibiting HIF-alpha prolyl hydroxylase. Cancer cell, 2005. 7(1): p. 77-85.

53. Hoekstra, A.S., et al., Parent-of-origin tumourigenesis is mediated by an essential imprinted modifier in SDHD-linked paragangliomas: SLC22A18 and CDKN1C are candidate tumour modifiers. Human molecular genetics, 2016

54. Tufton, N., et al., Outcomes of annual surveillance imaging in an adult and paediatric cohort of Succinate Dehydrogenase B mutation carriers (SDHB). Clinical endocrinology, 2016.

55. Linehan, W.M., et al., Comprehensive Molecular Characterization of Papillary Renal-Cell Carcinoma. The New England journal of medicine, 2016. 374(2): p. 135-45.

56. Sciacovelli, M., et al., Fumarate is an epigenetic modifier that elicits epithelial-to-mesenchymal transition. Nature, 2016. 537(7621): p. 544-547.

57. Lu, C., et al., IDH mutation impairs histone demethylation and results in a block to cell differentiation. Nature, 2012. 483(7390): p. 474-8.

58. Ohba, S., et al., Mutant IDH1 expression drives TERT promoter reactivation as part of the cellular transformation process. Cancer research, 2016. 6(22) p. 6680-6689.

59. Parsons, D.W., et al., An integrated genomic analysis of human glioblastoma multiforme. Science, 2008. 321(5897): p. 1807-12.

60. Wykoff, C.C., et al., Hypoxia-inducible expression of tumor-associated carbonic anhydrases. Cancer Res, 2000. 60(24): p. 7075-83.

61. Svastova, E., et al., Hypoxia activates the capacity of tumor-associated carbonic anhydrase IX to acidify extracellular pH. FEBS Lett, 2004. 577(3): p. 439-45.

62. Goto, Y., I. Nonaka, and S. Horai, A mutation in the tRNA(Leu)(UUR) gene associated with the MELAS subgroup of mitochondrial encephalomyopathies. Nature, 1990. 348(6302): p. 651-3.

63. El-Hattab, A.W., et al., MELAS syndrome: Clinical manifestations, pathogenesis, and treatment options. Mol Genet Metab, 2015. 116(1-2): p. 4-12.

64. Durham, S.E., et al., Normal levels of wild-type mitochondrial DNA maintain cytochrome c oxidase activity for two pathogenic mitochondrial DNA mutations but not for m.3243A-->G. American journal of human genetics, 2007. 81(1): p. 189-95.

65. Rossignol, R., et al., Mitochondrial threshold effects. The Biochemical journal, 2003. 370(Pt 3): p. $751-62$.

66. Jing, W., et al., Mitochondrial mutations associated with aminoglycoside ototoxicity and hearing loss susceptibility identified by meta-analysis. Journal of medical genetics, 2015. 52(2): p. 95-103.

67. Usami, S., et al., Prevalence of mitochondrial gene mutations among hearing impaired patients. Journal of medical genetics, 2000. 37(1): p. 38-40.

68. Shim, H., et al., c-Myc transactivation of LDH-A: implications for tumor metabolism and growth. Proceedings of the National Academy of Sciences of the United States of America, 1997. 94(13): p. 6658-63.

69. Quennet, V., et al., Tumor lactate content predicts for response to fractionated irradiation of human squamous cell carcinomas in nude mice. Radiother Oncol, 2006. 81(2): p. 130-5. 


\section{Chapter 8}

70. Blatt, S., et al., Lactate as a predictive marker for tumor recurrence in patients with head and neck squamous cell carcinoma (HNSCC) post radiation: a prospective study over 15 years. Clinical oral investigations, 2016. 20(8): p. 2097-2104.

71. Cairns, R.A., I.S. Harris, and T.W. Mak, Regulation of cancer cell metabolism. Nat Rev Cancer, 2011. 11(2): p. 85-95.

72. Wang, T.K., et al., Effects of carbonic anhydrase-related protein VIII on human cells harbouring an A8344G mitochondrial DNA mutation. Biochem J, 2014. 459(1): p. 149-60.

73. Rouschop, K.M., et al., The unfolded protein response protects human tumor cells during hypoxia through regulation of the autophagy genes MAP1LC3B and ATG5. J Clin Invest, 2010. 120(1): p. 127-41.

74. Springer, M.Z. and K.F. Macleod, In Brief: Mitophagy: mechanisms and role in human disease. J Pathol, 2016. 240(3): p. 253-255.

75. Hirschey, M.D., et al., SIRT3 regulates mitochondrial fatty-acid oxidation by reversible enzyme deacetylation. Nature, 2010. 464(7285): p. 121-5.

76. Palacios, O.M., et al., Diet and exercise signals regulate SIRT3 and activate AMPK and PGC-1alpha in skeletal muscle. Aging, 2009. 1(9): p. 771-83.

77. Fu, J., et al., trans-(-)-epsilon-Viniferin increases mitochondrial sirtuin 3 (SIRT3), activates AMP-activated protein kinase (AMPK), and protects cells in models of Huntington Disease. The Journal of biological chemistry, 2012. 287(29): p. 24460-72.

78. Qiu, X., et al., Calorie restriction reduces oxidative stress by SIRT3-mediated SOD2 activation. Cell Metab, 2010. 12(6): p. 662-7.

79. Qiao, A., et al., Sirt3-mediated mitophagy protects tumor cells against apoptosis under hypoxia. Oncotarget, 2016. 7(28): p. 43390-43400.

80. Finley, L.W., et al., SIRT3 opposes reprogramming of cancer cell metabolism through HIF1alpha destabilization. Cancer cell, 2011. 19(3): p. 416-28.

81. van Kuijk, S.J., et al., Prognostic Significance of Carbonic Anhydrase IX Expression in Cancer Patients: A Meta-Analysis. Front Oncol, 2016. 6: p. 69.

82. Dubois, L., et al., Specific inhibition of carbonic anhydrase IX activity enhances the in vivo therapeutic effect of tumor irradiation. Radiother Oncol, 2011. 99(3): p. 424-31.

83. van Kuijk, S.J., et al., The Sulfamate Small Molecule CAIX Inhibitor S4 Modulates Doxorubicin Efficacy. PLoS One, 2016. 11(8): p. e0161040.

84. Supuran, C.T. and J.Y. Winum, Carbonic anhydrase IX inhibitors in cancer therapy: an update. Future medicinal chemistry, 2015. 7(11): p. 1407-14.

85. Pastorek, J. and S. Pastorekova, Hypoxia-induced carbonic anhydrase IX as a target for cancer therapy: from biology to clinical use. Seminars in cancer biology, 2015. 31: p. 52-64.

86. Yuan, P., et al., Phenformin enhances the therapeutic benefit of BRAF(V600E) inhibition in melanoma. Proceedings of the National Academy of Sciences of the United States of America, 2013. 110(45): p. 18226-31.

87. Navarro, P., et al., Targeting Tumor Mitochondrial Metabolism Overcomes Resistance to Antiangiogenics. Cell reports, 2016. 15(12): p. 2705-18.

88. Zhang, L., H. He, and J.A. Balschi, Metformin and phenformin activate AMP-activated protein kinase in the heart by increasing cytosolic AMP concentration. American journal of physiology. Heart and circulatory physiology, 2007. 293(1): p. H457-66.

89. Miskimins, W.K., et al., Synergistic anti-cancer effect of phenformin and oxamate. PloS one, 2014. 9(1): p. e85576.

90. Zannella, V.E., et al., Reprogramming metabolism with metformin improves tumor oxygenation and radiotherapy response. Clinical cancer research : an official journal of the American Association for Cancer Research, 2013. 19(24): p. 6741-50.

91. Wheaton, W.W., et al., Metformin inhibits mitochondrial complex I of cancer cells to reduce tumorigenesis. eLife, 2014. 3: p. e02242.

92. Hawley, S.A., et al., Calmodulin-dependent protein kinase kinase-beta is an alternative upstream kinase for AMP-activated protein kinase. Cell metabolism, 2005. 2(1): p. 9-19. 
93. Cheng, G., et al., Mitochondria-Targeted Analogues of Metformin Exhibit Enhanced Antiproliferative and Radiosensitizing Effects in Pancreatic Cancer Cells. Cancer research, 2016. 76(13): p. 3904-15.

94. Mountzios, G., G. Pentheroudakis, and P. Carmeliet, Bevacizumab and micrometastases: revisiting the preclinical and clinical rollercoaster. Pharmacology \& therapeutics, 2014. 141(2): p. 117-24.

95. Hu, Y.L., et al., Hypoxia-induced autophagy promotes tumor cell survival and adaptation to antiangiogenic treatment in glioblastoma. Cancer research, 2012. 72(7): p. 1773-83.

96. Steinhorn, B.S., J. Loscalzo, and T. Michel, Nitroglycerin and Nitric Oxide--A Rondo of Themes in Cardiovascular Therapeutics. N Engl J Med, 2015. 373(3): p. 277-80.

97. Yasuda, H., et al., Randomized phase II trial comparing nitroglycerin plus vinorelbine and cisplatin with vinorelbine and cisplatin alone in previously untreated stage IIIB/IV non-small-cell lung cancer. Journal of clinical oncology : official journal of the American Society of Clinical Oncology, 2006. 24(4): p. 688-94.

98. Arrieta, O., et al., Phase II study. Concurrent chemotherapy and radiotherapy with nitroglycerin in locally advanced non-small cell lung cancer. Radiotherapy and oncology : journal of the European Society for Therapeutic Radiology and Oncology, 2014. 111(2): p. 311-5.

99. Dingemans, A.M., et al., A randomized phase II study comparing paclitaxel-carboplatin-bevacizumab with or without nitroglycerin patches in patients with stage IV nonsquamous nonsmall-cell lung cancer: NVALT12 (NCT01171170)dagger. Annals of oncology : official journal of the European Society for Medical Oncology, 2015. 26(11): p. 2286-93.

100. Brown, G.C. and C.E. Cooper, Nanomolar concentrations of nitric oxide reversibly inhibit synaptosomal respiration by competing with oxygen at cytochrome oxidase. FEBS letters, 1994. 356(2-3): p. 295-8.

101. Dungel, P., et al., Neither nitrite nor nitric oxide mediate toxic effects of nitroglycerin on mitochondria. Journal of biochemical and molecular toxicology, 2011. 25(5): p. 297-302.

102. Caneba, C.A., et al., Nitric oxide is a positive regulator of the Warburg effect in ovarian cancer cells. Cell death \& disease, 2014. 5: p. e1302.

103. McAllister, A., et al., Plasma levels of nitroglycerin generated by three nitroglycerin patch preparations, Nitradisc, Transiderm-Nitro and Nitro-Dur and one ointment formulation, Nitrobid. British journal of clinical pharmacology, 1986. 21(4): p. 365-9.

104. Reddy, S.B. and S.K. Williamson, Tirapazamine: a novel agent targeting hypoxic tumor cells. Expert opinion on investigational drugs, 2009. 18(1): p. 77-87.

105. Patterson, A.V., et al., Mechanism of action and preclinical antitumor activity of the novel hypoxiaactivated DNA cross-linking agent PR-104. Clinical cancer research : an official journal of the American Association for Cancer Research, 2007. 13(13): p. 3922-32.

106. Guise, C.P., et al., The bioreductive prodrug PR-104A is activated under aerobic conditions by human aldo-keto reductase 1C3. Cancer research, 2010. 70(4): p. 1573-84.

107. Peeters, S.G., et al., TH-302 in Combination with Radiotherapy Enhances the Therapeutic Outcome and Is Associated with Pretreatment [18F]HX4 Hypoxia PET Imaging. Clinical cancer research : an official journal of the American Association for Cancer Research, 2015. 21(13): p. 2984-92.

108. Meng, F., et al., Molecular and cellular pharmacology of the hypoxia-activated prodrug TH-302. Molecular cancer therapeutics, 2012. 11(3): p. 740-51.

109. Chawla, S.P., et al., Phase II study of the safety and antitumor activity of the hypoxia-activated prodrug TH-302 in combination with doxorubicin in patients with advanced soft tissue sarcoma. Journal of clinical oncology : official journal of the American Society of Clinical Oncology, 2014. 32(29): p. 3299-306.

110. Teicher, B.A., J.S. Lazo, and A.C. Sartorelli, Classification of antineoplastic agents by their selective toxicities toward oxygenated and hypoxic tumor cells. Cancer research, 1981. 41(1): p. 73-81.

111. Vaupel, P., O. Thews, and M. Hoeckel, Treatment resistance of solid tumors - Role of hypoxia and anemia. Medical Oncology, 2001. 18(4): p. 243-259.

112. Holohan, C., et al., Cancer drug resistance: an evolving paradigm. Nature reviews. Cancer, 2013. 13(10): p. 714-26.

113. Townsend, D.M. and K.D. Tew, The role of glutathione-S-transferase in anti-cancer drug resistance. Oncogene, 2003. 22(47): p. 7369-75. 


\section{Chapter 8}

114. Johansson, K., et al., Characterization of new potential anticancer drugs designed to overcome glutathione transferase mediated resistance. Molecular pharmaceutics, 2011. 8(5): p. 1698-708.

115. Trachootham, D., J. Alexandre, and P. Huang, Targeting cancer cells by ROS-mediated mechanisms: a radical therapeutic approach? Nature reviews. Drug discovery, 2009. 8(7): p. 579-91.

116. Traverso, N., et al., Role of glutathione in cancer progression and chemoresistance. Oxidative medicine and cellular longevity, 2013. 2013: p. 972913.

117. Urig, S. and K. Becker, On the potential of thioredoxin reductase inhibitors for cancer therapy. Seminars in cancer biology, 2006. 16(6): p. 452-65.

118. Deponte, M., Glutathione catalysis and the reaction mechanisms of glutathione-dependent enzymes. Biochimica et biophysica acta, 2013. 1830(5): p. 3217-66.

119. Advantages of personalized cancer therapy. [Web Page] 2016 11/16/2016]; Available from: http://personalizedtherapy.eu/advantages.

120. Gabizon, A., et al., Prolonged circulation time and enhanced accumulation in malignant exudates of doxorubicin encapsulated in polyethylene-glycol coated liposomes. Cancer research, 1994. 54(4): p. 987-92.

121. Mayer, A., et al., A phase I study of single administration of antibody-directed enzyme prodrug therapy with the recombinant anti-carcinoembryonic antigen antibody-enzyme fusion protein MFECP1 and a bisiodo phenol mustard prodrug. Clin Cancer Res, 2006. 12(21): p. 6509-16.

122. Mukhopadhyay, P., et al., Mitochondrial-targeted antioxidants represent a promising approach for prevention of cisplatin-induced nephropathy. Free radical biology \& medicine, 2012. 52(2): p. 497-506.

123. Buondonno, I., et al., Mitochondria-Targeted Doxorubicin: A New Therapeutic Strategy against Doxorubicin-Resistant Osteosarcoma. Molecular cancer therapeutics, 2016.

124. Orloff, J., et al., The future of drug development: advancing clinical trial design. Nature reviews. Drug discovery, 2009. 8(12): p. 949-57.

125. Dubois, L.J., et al., New ways to image and target tumour hypoxia and its molecular responses. Radiotherapy and oncology : journal of the European Society for Therapeutic Radiology and Oncology, 2015. 116(3): p. 352-7. 
Summary 

Currently cancer is still one of the deadliest diseases. Effective treatment of solid malignancies is dependent on the tolerability of normal tissues and its effectiveness to fight the malignant cells. A cancer treatment that reduces at the same time treatment resistance and normal tissue toxicity is highly desirable. Multiple hallmarks of cancer have been described which influence tumorigenesis and treatment response. Deregulation of cellular energetics is one of these hallmarks and the topic of this thesis. Under normal physiological conditions mitochondria play an important role in the regulation of cellular metabolism and therefore they are often referred to as the "power plants of a cell'. Besides nuclear genes also 37 genes located in the mitochondria's own DNA (mtDNA) are involved in the regulation of mitochondrial function and cellular respiration. Mitochondria and mtDNA are heterogeneous and dynamic on different levels. Mutations in mtDNA can for instance be present only in a subpopulation of the mtDNA, and as every mitochondrion can contain multiple copies of mtDNA this can lead to a different distribution of mutations in tissues. Additionally, mitochondria play a very dynamic role in various processes, as they are able to use fusion and fission to maintain functional mitochondria upon metabolic and/or environmental stress. Several associations have been found between altered mitochondrial function and tumorigenesis and even cancer treatment.

The aim of this thesis was to investigate the role of mitochondrial function, reactive oxygen species (ROS) and hypoxia modulation in tumors in view of the possibility of implementing them in a personalized treatment approach. Several molecular approaches have been used to investigate the effect of energetic stress and/or stress inducers (irradiation and hypoxia) in relation to mitochondrial function (chapter 3 and 4). In chapter 3 we evaluated the effect of full mtDNA depletion, often used as an extreme model to investigate mitochondrial dysfunction, on radiotherapy response. Here, we found that mtDNA depleted cells derived from a variety of cell lines displayed a different response upon radiation, a phenomenon that has been previously reported by others. The antioxidant capacity of cells, in this case represented by decreased ratio of GSH:GSSG and mitochondrial super oxide dismutase (SOD2), could possibly explain a reduced response upon irradiation for different cell types and potentially influence the response towards radiotherapy in these cellular models. Hypoxia forms a well-known limitation for effective radiotherapy and corresponds with a decreased prognosis. Normally under hypoxic conditions, cells make a shift to glycolysis (the crab-tree effect) and show an increased extracellular acidification. Carbonic anhydrase (CA) IX is a hypoxia-regulated enzyme involved in maintaining the balance between a physiological intracellular $\mathrm{pH}$ and extracellular acidification. In chapter 4 we investigated the effect of mitochondrial dysfunction on CAIX in 3 different manners. The induction of mitochondrial dysfunction by depleting the mitochondrial own genome, introduction of a mtDNA point mutation (normally inducing mitochondrial encephalomyopathy, lactic acidosis, and stroke-like episodes" (MELAS)) and pharmacological inhibition of complex 
(C) I of the oxidative phosphorylation (OXPHOS) led to a decreased CAIX expression (both protein and mRNA) under hypoxic conditions. Similar results were obtained for another HIF-1 $\alpha$ downstream target, VEGF. In general, HIF-1 $\alpha$ protein levels themselves were reduced upon hypoxia after the induction of a mitochondrial dysfunction, suggesting involvement of the oxidative phosphorylation in HIF-1 $\alpha$ stabilization. However, the precise mechanisms still have to be further elucidated.

Furthermore, different drug strategies (dual-targeting CAIX phenformin, repurposing nitroglycerin or prodrugs such as ANS-DOX) were investigated for the ability of influencing different cancer treatment limiting parameters such as toxicity, hypoxia and drug resistance (chapter 5, 6 and 7). CAIX is upregulated in several tumors and its expression is correlated with a worse patient prognosis. Therefore, it can be exploited for targeting purposes of drugs, thereby reducing their potential side effects. In chapter 5 we used this approach to target both CAIX and the $\mathrm{Cl}$ of the OXPHOS by using a compound related to the biguanide metformin. Although phenformin is a more potent compound than metformin it has an unfavorable toxicity profile, but therefore it is an interesting drug to use for a dual-targeting approach. The phenformin CAIXi conjugated derivative 18 has good binding affinities to CAIX and CAXII, however, the functionality of the compound in respect to inhibiting OXPHOS was absent in our experiments. It has been described that inhibition of OXPHOS could provide a better oxygenation of the tumor as oxygen consumption is reduced. Another approach to improve the oxygen status of the tumor would be by modulating the perfusion of the tumor. Nitroglycerin, normally used to treat angina pectoris, releases nitric oxide and exhibits vasodilating properties. In chapter 6 we observed a decreased hypoxic fraction after application of a nitroglycerin patch in tumors upfront characterized as being a 'hypoxic tumor' using HX4 hypoxia PET imaging. In these hypoxic tumors, the HX4 tracer uptake negatively correlated with perfusion. In vitro, we elucidated that the metabolism of the 2nitroimidazole HX4 is not influenced by nitroglycerin itself and that nitroglycerin does not inhibit mitochondrial respiration, as previously has been suggested by others at doses achievable in humans. Taken together, this could suggest that the additional application of nitroglycerin for the treatment of hypoxic tumor could be beneficial, although further studies need to be performed.

Next to a dual-targeting (chapter 5) and drug repurposing (chapter 6) approach also a prodrug approach was tested. This prodrug approach is based on the principle that the prodrug is enzymatically activated under certain circumstances. In this thesis, a prodrug approach was evaluated in order to investigate the possibility of overcoming drug resistance. Unfortunately, many cancer patients suffer from resistance towards chemotherapeutics and thereby their treatment is less effective. Mechanisms involved in glutathione metabolism are often related to drug resistance, a phenomenon that is not exclusively observed in hypoxic tumors. Glutathione transferases (GST) are a class of enzymes involved in detoxification of substances and are often overexpressed in tumors 
contributing to a drug resistant phenotype. GST mediated drug resistance could potentially be overcome by using a prodrugs approach. In chapter 7, we investigated prodrug formulations of two widely used chemotherapeutics, etoposide and doxorubicin, to overcome GST mediated resistance. For microsomal glutathione transferase (MGST1, found in the outer membrane of the mitochondria and in the membrane of the endoplasmic reticulum) overexpressing cells we observed that, 4acetyl-2-nitro-benzenesulfonyl etoposide (ANS-etoposide) (showed a MGST1 dependent therapeutic efficacy. However, this effect was only slightly dose dependent and therapeutic efficacy was lower compared to the parental drug. This suggests that the modification reduced either the uptake of the drug or limited its activation. Overexpression of GSTA1-1, another subclass of GSTs, did show a resistant phenotype towards etoposide which could be counteracted by ANS-etoposide, suggesting that GSTA1-dependent activation of the prodrug took place. For doxorubicin, a drug known for its cardiac toxicity as a side effect, we showed that different sulfonamide moieties have an optimal GST dependent window and can be optimally activated by certain subclasses of GSTs. These prodrugs selectively target GST overexpressing cells, while limiting normal tissue side effects at the same time. However, not only GSTs interacted and were inhibited by the doxorubicin based prodrugs as thioredoxin reductase 1 (TrxR1), another important antioxidant enzyme in the thioredoxin (Trx) system, was also targeted by using these prodrugs. Influencing both the glutathione and the Trx system, these prodrugs and the concurrent effects caused by them provide us with a promising approach of targeting chemoresistant tumors.

In the different studies described within this thesis, we observed that modulation of mitochondrial function and mitochondrial proteins can contribute to alterations of treatment responses. This provides us with new insights in the involvement of mitochondrial function and mitochondrial proteins in cancer therapy. However, as tumor tissues are very heterogeneous and adaptive towards their environment, patient stratification is essential to see which subpopulations of patients would by susceptible for such treatment modulation. 

Samenvatting 

Kanker is tegenwoordig een van de meest dodelijke ziektes. Effectieve behandeling van solide tumoren is afhankelijk van het toelaatbare effect op het normale weefsel. De behandeling dient echter ook effectief genoeg te zijn om de kwaadaardige cellen te bestrijden. Momenteel is het noodzakelijk om de kankerbehandeling effectiever te maken, waarbij therapie resistentie van de tumoren wordt aangepakt, maar waarbij ook de normale weefsels gespaard worden. In het verleden zijn er verschillende eigenschappen van tumoren beschreven die de vorming van tumoren en het effect van kankerbehandelingen beïnvloeden. Een van deze eigenschappen is het ontregelen van het metabolisme van een cel. Onder normale fysiologische omstandigheden spelen mitochondriën vaak een belangrijke rol in het regelen van het metabolisme van een cel. Vanwege deze rol worden ze vaak beschreven als de energiefabrieken van een cel. Tot op heden zijn verschillende verbanden gevonden tussen de veranderingen in de functie van deze mitochondriën, de vorming van tumoren en kankerbehandeling. Naast de nucleaire genen zijn er ook 37 genen, aanwezig in het eigen DNA van de mitochondriën (mtDNA), betrokken bij het regelen van de mitochondriële functie en oxidatieve fosforylatie (OXPHOS). Mitochondriën en mtDNA zijn heterogeen en dynamisch van aard op verschillende niveaus. Mutaties in mtDNA kunnen bijvoorbeeld alleen aanwezig zijn in een subpopulatie van de mtDNA moleculen en aangezien elk individueel mitochondrion meerdere kopieën van het mtDNA kan bevatten, kan dit leiden tot een verschillende distributie van mutaties in weefsels. Tevens spelen mitochondriën een dynamische rol in meerdere processen, omdat ze in staat zijn om 'fusion en fission' (versmelting en deling) toe te passen om hun functionaliteit te behouden als cellen metabolische en/of andere vormen van stress ervaren. De rol van de mitochondriën en de interactie met het metabolisme gedurende de behandeling zijn een fascinerend onderzoeksveld.

Het doel van deze studie was het onderzoeken van de rol en bijdrage van de mitochondriële functie, reactieve zuurstofverbindingen (ROS) en hypoxie veranderingen in tumoren en hoe deze toe te passen in een persoonlijk behandelingsplan van een patiënt. Moleculair biologische experimenten werden uitgevoerd om de relatie tussen de mitochondriële functie en het effect van energetische stress en/of veroorzakers van stress (zoals hypoxie en bestraling) te onderzoeken (hoofdstuk 3 en 4). Als eerste evalueerden we het effect van volledige verwijdering van het mtDNA (depletie) op bestraling. mtDNA depletie wordt vaak gebruikt als een extreem model om de invloed van de mitochondriële dysfunctie te onderzoeken. In hoofdstuk 3 observeerden we dat verschillende cellijnen ontdaan van hun mtDNA andere effecten hebben na bestraling, een observatie die eerder ook door anderen werd gedaan. De antioxidant capaciteit van cellen, hier weergegeven door een afname in de ratio van GSH:GSSG en mitochondriële superoxide dismutase (SOD2), kan dit mogelijk beïnvloeden en verklaren waarom een verminderd effect na bestraling plaatsvindt in bepaalde celmodellen. Voor effectieve radiotherapie is hypoxie een bekende beperkende factor die gerelateerd is aan een 
verminderde prognose voor patiënten. Normaal gezien maken cellen onder hypoxische condities een verandering van hun metabolisme door en stappen ze over op glycolyse, het zo genoemde "crab-tree effect", waarbij meer verzuring buiten de cel optreedt. Koolzuur anhydrase 9 (CAIX) is een enzym dat gereguleerd wordt door hypoxie en betrokken is bij het onderhouden van de balans tussen een fysiologische zuurtegraad binnen en verzuring buiten de cel. In hoofdstuk 4 onderzochten we op 3 verschillende manieren het effect van mitochondriële dysfunctie op CAIX. Het veroorzaken van een mitochondriële dysfunctie door het verwijderen van het mtDNA, het introduceren van een punt mutatie in het mtDNA (hetgeen "mitochondrial encephalomyopathy, lactic acidosis, en stroke-like (=beroerteachtige) episodes" (MELAS) veroorzaakt) en farmacologische inhibitie van complex I van de OXPHOS leiden tot een verminderde CAIX-expressie (op zowel eiwit- als mRNA niveau) onder hypoxische condities. Ook een ander eiwit dat wordt beïnvloed door HIF-1 $\alpha$, VEGF, vertoonde een verlaagde expressie. Over het algemeen waren ook de HIF-1 $\alpha$ eiwitniveaus verlaagd na de inductie van de mitochondriële dysfunctie. Dit suggereert dat verlaging van HIF-1 $\alpha$ eiwitniveaus wellicht kunnen worden veroorzaakt door een verminderde OXPHOS-functie. Echter het exacte mechanisme moet nog verder onderzocht worden.

Tevens werden in deze thesis verschillende manieren van innovatieve medicatietoepassingen onderzocht (dual-targeting CAIX fenformine, een ander gebruik dan de huidige toepassing van nitroglycerine, en prodrugs zoals 4-acetyl-2-nitrobenzenesulfonyl doxorubicine (ANS-DOX) op effectiviteit in het beïnvloeden van verschillende parameters die een nadelige invloed hebben op kankerbehandelingen zoals toxiciteit, hypoxie en resistentie tegen de medicatie (hoofdstuk 5, 6 en 7). CAIXexpressie is verhoogd in verschillende tumoren en deze verhoogde expressie houdt verband met een slechtere prognose voor patiënten. Door deze eigenschappen is CAIX een interessant doelwit zodat bepaalde medicatie specifiek gericht tegen CAIX naar de tumor gebracht kan worden. Hierdoor kunnen mogelijke bijwerkingen van de medicatie worden verminderd. In hoofdstuk 5 gebruikten we deze aanpak om tegelijkertijd de activiteit van CAIX en complex I van de OXPHOS te remmen. Hiervoor gebruikten we een compound die afgeleid is van metformine, namelijk fenformine. Ondanks dat fenformine een krachtigere werking heeft dan metformine, heeft het ook een minder goed toxiciteitsprofiel. Dit maakt fenformine echter een interessant molecule voor onze strategie gebaseerd op 'dual-targeting'. Het fenformine CAIXi geconjugeerde derivaat 18 liet een goede bindingsaffiniteit zien met CAIX en CAXII, echter de functionaliteit van het molecule met betrekking tot het remmen van OXPHOS was afwezig in onze experimenten. In de literatuur is beschreven dat OXPHOS-remming kan zorgen voor een betere zuurstofstatus van de tumor, omdat het zuurstofverbruik is verminderd. Het verbeteren van de perfusie van de tumor zou mogelijk een andere manier zijn om de zuurstofstatus van de tumor te beïnvloeden. Nitroglycerine, een medicijn dat normaal wordt gebruikt voor de behandeling van angina pectoris, geeft stikstofmonoxide af en 
zorgt voor vaatverwijding (vasodilatatie). Na gebruik van een nitroglycerine pleister observeerden we een afname in de hypoxische fractie enkel van de hypoxische tumoren (hoofdstuk 6). Hypoxie is een van de belangrijke limiterende factoren voor de behandeling van tumoren. In hypoxische tumoren is na het gebruik van een nitroglycerine pleister een omgekeerd verband tussen de opname van de hypoxie merker HX4, welke bedoeld is om de hoeveelheid hypoxie binnen een tumor te bepalen, en perfusie. Door middel van in vitro experimenten konden we uitsluiten dat het metabolisme van 2-nitromidazoles, waar HX4 er een van is, niet beïnvloed werd door nitroglycerine zelf. Tevens konden we uitsluiten dat de mitochondriële respiratie werd verminderd door de blootstelling van nitroglycerine aan een concentratie gevonden in het plasma van patiënten. De toevoeging van nitroglycerine aan de behandeling van hypoxische tumoren lijkt daarom veelbelovend, echter verder onderzoek is nog nodig.

Naast het gebruik van een dual-targeting benadering (hoofdstuk 5) en het innovatieve herbestemming van medicijnen (hoofdstuk 6) werd ook het gebruik van een activeerbaar medicijn (prodrug) getest. In deze thesis werd deze benadering gebruikt om te onderzoeken of het mogelijk was om resistentie tegen bepaalde medicatie te ondervangen. Helaas hebben vele kankerpatiënten last van resistentie tegen chemotherapie waardoor hun behandeling minder effectief is, met alle gevolgen van dien. Mechanismen welke betrokken zijn bij het glutathion metabolisme zijn vaak gerelateerd aan resistentie tegen medicatie en zijn een fenomeen dat niet exclusief aanwezig is bij hypoxische tumoren. Glutathion transferases (GSTs) zijn een klasse van enzymen betrokken bij de detoxificatie van stoffen en komen vaak tot overexpressie in tumoren. In verschillende type tumoren zijn GSTs gelinkt aan de resistentie tegen medicatie bij kankerpatiënten. Mogelijk kan door het gebruik van prodrugs de GST gemedieerde medicijnresistentie overwonnen worden. In hoofdstuk 7 gebruikten we prodrugs gebaseerd op 2 vaak gebruikte chemotherapieën, namelijk etoposide en doxorubicine, om GST gemedieerde resistentie te overwinnen. Bij cellen die microsomale glutathion transferase 1 (MGST1, aanwezig in de buitenste membraan van het mitochondrion en in het membraan van het endoplasmatisch reticulum) tot overexpressie brengen, zagen we voor ANS-etoposide een MGST1 afhankelijke toename van de therapeutische doeltreffendheid. Echter deze toename was enkel beperkt afhankelijk van de toegevoegde concentratie en leidde tot een mindere efficiëntie vergeleken met de oorspronkelijke niet-gemodificeerde medicatie. Dit suggereert dat de modificatie leidde tot verminderde opname en/of tot verminderde activatie. Overexpressie van GSTA1-1, een andere subklasse van GSTs, liet een resistent fenotype tegenover etoposide zien die teruggebracht kon worden door het gebruik van ANSetoposide. Dit suggereert dat GSTA1-1 afhankelijke activatie van de prodrug aanwezig was. Voor doxorubicine, een medicijn dat bekend staat voor het veroorzaken van cardiotoxiciteit bij patiënten, lieten we zien dat verschillende sulfonamide restgroepen 
kunnen zorgen voor een optimaal GST afhankelijk venster. De verschillende vormen van de prodrugs kunnen optimaal geactiveerd worden door verschillende subklasses van GSTs en selectief werken op de cellen met een GST overexpressie. Hierdoor kunnen bijwerkingen op normaal weefsel verminderd worden. In deze studie observeerden we echter dat niet alleen GSTs een interactie aangingen met de prodrugs, maar dat ook een ander belangrijk antioxidant enzym (thioredoxine reductase; TrxR1) in het thioredoxine systeem werd beïnvloed door de prodrugs. Het beïnvloeden van zowel het glutathion als het Trx systeem zorgt voor een verhoogd effect en is daardoor een veelbelovende aanpak voor de behandeling van chemoresistente tumoren.

In de verschillende studies beschreven in deze thesis observeerden we dat het beïnvloeden van de mitochondriële functie en mitochondriële eiwitten kan bijdragen tot de verandering van het effect van de behandeling. Dit zorgt voor nieuwe inzichten in de betrokkenheid van mitochondriële functie en mitochondriële eiwitten in kankertherapie. Omdat echter tumorweefsels erg divers zijn en zich makkelijk kunnen aanpassen aan veranderende omstandigheden en omgeving, is de selectie van patiënten die baat hebben bij de aangepaste behandelingsmethoden echter van groot belang. 
Valorization addendum 

In this thesis, the scientific relevance of modulation of mitochondrial DNA and the role of mitochondria in the context of cancer treatment has been demonstrated. Scientific knowledge and findings should eventually benefit the society in one or another way. Worldwide, cancer is now-a-days one of the leading causes of death. In 2015, 104988 new patients were diagnosed with cancer in the Netherlands [1]. Next to the emotional burden of such a disease also the financial aspect plays an important role for patients and society. In the Netherlands, cancer treatment costs were already 4,8 billion euro in 2011 [2] and are rising every year. Currently, in our society a debate is ongoing on health and insurance policy in order to control the costs of cancer treatments combined with an improvement of survival rates and quality of life [3]. As not every patient is the same and there is not yet a perfect cancer therapy, stratification of patients is an important tool to select only the patients who will benefit the most from a certain (combination) treatment in order to reduce costs.

\section{Clinical relevance and improvement for health care}

As tumors are highly dynamic and heterogeneous in their appearance, treatment should be tailored to the specific needs of a patient; the so-called personalized treatment approach. Currently cancer researchers try to find better biomarkers to predict the patients' outcome to a specific treatment or to use as target for treatment. Mitochondrial function (chapter 2, 3 and 4), CAIX (chapter 4 and chapter 5), hypoxia (chapter 5 and 6) and GST expression (chapter 8) could serve as such biomarkers. Complementary to the use of specific drugs to eliminate cancer, possible normal tissue toxicity plays an important role in defining the optimal treatment approach. In lung cancer patients for example, the healthy tissue tolerance defines the radiation dose delivered to the tumor. Therefore, it is important to develop methods that can identify patients at risk for e.g. radiation-induced lung toxicity (RILT). Radiation therapy (RT) and mitochondria have common molecular processes. Where mitochondria are important for energy (ATP) and reactive oxygen species (ROS) production, regulation of apoptosis and cellular calcium homeostasis, RT induces the formation of ROS and can lead to cell death. Recently, it has been shown that ionizing radiation can elevate mitochondrial content and function. Furthermore, mitochondrial DNA (mtDNA) mutations have previously been reported to affect mitochondrial function (decreased ATP production, increased ROS production). As described in chapter 2 and 3, evaluation of the mitochondrial status and their functionality could result in a biomarker that helps us to identify new therapeutic targets, potentially limiting normal tissue toxicity or increasing tumor specific efficacy and provide us with better working therapies at the same time although further progress is necessary. Potentially this could not only work for radiotherapy as treatment modality, but could also be expanded to other treatments 
like immunotherapy or chemotherapy and thereby contribute to a more optimal treatment of patients.

CAIX has been found to influence disease progression and is associated with a higher risk to develop metastasis. As CAIX is seen as an endogenous hypoxia marker since its expression is often HIF-1 $\alpha$ regulated, identification of hypoxic areas could be beneficial on several distinctive levels. Different approaches could be pursued to increase the effectiveness of treatments by manipulating the tumoral hypoxic fraction. Drugs such as nitroglycerin, metformin or phenformin are suggested to reduce the hypoxic fraction within tumors. The CAIX inhibitors described in chapter 5 were shown not to be effective as single agents or didn't result in an enhanced efficiency compared to their parental compounds and to standard treatment modalities. Therefore, additional studies were not pursued. The combination of another biguanide metformin with radiotherapy is currently tested in clinical trials and recently mitochondrial targeted derivatives of metformin have been developed. Targeting a biguanide to mitochondria and making it more effective and consequently reduce side effects remains an interesting research area. These newly designed compounds are believed to be more effective than metformin itself and could therefore contribute to a beneficial therapy. Although these types of compounds require further extensive preclinical and clinical testing, the first results are promising. However, until they are proven more effective and equally safe as the parental drug, metformin would currently be the way to go forward.

In chapter 6, we indeed observed a decreased hypoxic fraction upon nitroglycerin administration in patients harboring hypoxic tumors. Since hypoxia causes resistance to radiotherapy, reducing the hypoxic fraction in these treated patients is of great importance. As metformin and nitroglycerin are FDA approved drugs, we know the possible side effects when patients are treated with these drugs. Therefore, these agents could be used relatively safe for repurposing. Of course, the effect on the tumor and its microenvironment still need to be carefully elucidated in patients when combined with conventional treatment modalities; so adverse tumor effects could be prevented. Since nitroglycerin reduced the hypoxic fraction only in patients with hypoxic tumors, assessing if a tumor is hypoxic is essential. Multiple hypoxia tracers and imaging modalities are currently available; however, it is important to validate these tracers in the same model systems to acquire advantages and limitations of each imaging modality. During our nitroglycerin study, we observed that performing several scans using different imaging modalities (HX4 (hypoxia PET/CT), DCE-CT (perfusion CT), FDG PET/CT) increased the burden on patients and physicians excessively resulting in premature termination of the study. Furthermore, we observed that it is utmost important to stratify patients to increase the chance of an effective non-small cell lung cancer (NSCLC) treatment after application of nitroglycerin. 


\section{Gain for society}

The observations made in this thesis could potentially benefit cancer patients and the society in general. Although at first sight the introduction of a personalized medicine approach sounds like an expensive one. But unfortunately, from the economic point of view, a "one size fits all" strategy in cancer treatment does not lead to a favorable treatment outcome for the majority of patients. Therefore, an improvement in treatment response with low developmental costs can be considered as a good strategy. Repurposing of already FDA approved drugs or even the targeted re-use of drugs withdrawn from the market in the past due to toxicity problems in other diseases, could be interesting approaches. Also, implementing drugs in cancer treatment strategies with a known toxicity profile from other diseases would be an interesting approach. In this thesis, we have demonstrated that, with a correct patient stratification, previously developed drugs such as nitroglycerin (chapter 6) could reduce treatment limiting aspects, like for instance hypoxia. Derivatives of drugs such as phenformin or doxorubicin (chapter 5 and 7) in order to target specific areas of a tumor or to modulate their toxicity profile would help us to reduce the costs for society. Using this approach, investigating new potential targets and developing specific therapeutic agents for these targets with unknown toxicity profiles can be prevented. This would reduce the development costs of new treatments and eventually reduce the costs of a certain treatment. Repurposing of drugs is an approach that also gained the interest of the scientific community as it creates opportunities for new research. Gaining more knowledge on the biological processes involved in certain types of treatment as well as their functional consequences benefits not only researchers but also physicians and thereby patients in the long run. Patients however should be more often made aware of the fundamental and translational research essential to improve the treatment of their diseases.

\section{Road to the market}

Although it is still a long road, the studies performed in this thesis do have the potential to be implemented in daily practice and additional studies to prove the efficiency and safety are the next steps to be performed. The dual-targeting agents described in this thesis remain to be further developed with respect to efficacy and safety, as the ones described in this thesis were found not to be effective enough. On the other hand, CAIX expression levels themselves are also interesting for further research. Prior to developing a dual-targeting agent, used for homing a drug to the tumor, CAIX expression levels itself could be used as a biomarker for malignant and invasive tumors and thereby for predicting treatment outcome. Further preclinical and clinical research however is necessary before introducing such a biomarker, imaging agents and/or CAIX 
targeting agents into clinical practice. Similarly, also the GST prodrugs investigated in the thesis need further evaluation and validation regarding efficacy, efficiency and safety for their use as a treatment for chemoresistant tumors. Previous developed GST activated prodrugs such as TLK-286 (Telcyta, canfosfamide) enrolled already into clinical trials, thereby demonstrating the potency of this approach.

For drugs already preclinically used in a safe manner, window-of-opportunity clinical trials can elucidate efficacy in patients in a cost-effective manner. Using this approach, non-effective adjuvant drugs will be filtered out in an earlier development phase instead of going through conventional clinical trials and therefore costs will be reduced. Adjuvant drugs will be evaluated for their specific therapeutic effects as monotherapy (e.g. reducing hypoxia after application), before combining them with standard therapy. When a drug is found to be effective and potentially interesting to continue, clinical trials should be performed. Nitroglycerin has been evaluated already in clinical trials as adjuvant NSCLC treatment, but didn't live up to the expectations, explained by the lack of patient stratification. In our study (chapter 6) we showed that there is an opportunity for the use of nitroglycerin in a subset of patients, namely those patients with hypoxic tumors. Since nitroglycerin targets tumor hypoxia, we believe that patient stratification based on hypoxia imaging is crucial for these types of drugs. Hypoxia imaging is already used in clinical settings and can be exploited for specific targeting of these hypoxic areas in tumors or for guiding proper patient stratification. Taking along better and less invasive patient stratification methods during drug development, will reduce toxicity and increase efficacy for the patient and will eventually lead to a significant cost reduction for the society.

In the future, treatments will become more tailor-made and more specific based on the patients' needs, although the financial pressure on our health system keeps on increasing. Improvement of patient stratification can eliminate unnecessary and unsuccessful treatments and will thereby reduce treatment costs and therapy burden for patients. Investing in modalities to improve stratification is therefore essential. Additionally, drug repurposing whether or not in combination with drug modifications for potential new treatment approaches could be a valid approach to reduce drug development costs and hopefully reduces the burden on our health system.

\section{References}

1. Nederlandse Kankerregistratie. webpage cited: 2016-09-30; Available from: http://www.cijfersoverkanker.nl.

2. Kosten van Ziekten. webpage cited: 2016-11-27; Available from: https://kostenvanziektentool.volksgezond heidenzorg.info/tool/nederlands/.

3. Rusman, F., Hoeveel mag een mensenleven kosten? NRC Handelsblad, 2015-11-14. 
Acknowledgements / Dankwoord 
Dan komt nu echt het allerlaatste, maar waarschijnlijk het eerste zo niet het enige, dat het overgrote gedeelte van jullie van mijn proefschrift lezen. Kortom ik heb maar één kans om het goed te doen, niemand te vergeten en hopelijk lukt dat een beetje, al heb ik het voorgevoel dat ik dat heel snel los moet laten. Maar misschien is dit wel de handigste manier om toch iedereen te bedanken en niemand te vergeten: Bedankt allemaal!

Beste Philippe, bedankt voor de mogelijkheid die je me gegeven hebt om mijn PhD te doen bij Maastro. De compleet uiteenlopende onderwerpen binnen onze projectgroep weet jij feilloos met elkaar te combineren, waardoor ik als AlO ook veel van andere onderwerpen heb kunnen leren.

Ook mijn 'Belgische' co-promotores wil ik natuurlijk hartelijk bedanken voor de uitbreiding van mijn Vlaamse vocabulaire en de begeleiding bij mijn promotietraject.

An, ondertussen ben je al even weg uit Maastricht, maar dat wil niet zeggen dat je minder betrokken bent gebleven bij mijn promotieonderzoek. Dank je wel dat je altijd tijd en aandacht voor me had. Tegenwoordig wordt elke vraag beantwoord via de moderne communicatiemiddelen (telefoonconferenties, Whatsapp, email of Skype, welke hebben we niet gebruikt), maar dat doet geen afbreuk aan je betrokkenheid. Ik ga je $(:) ;$ missen.

Ludwig, de zin "mijn deur staat altijd open", heb ik goed in mijn oren geknoopt. Ik weet nog goed dat ik altijd even snel een vraag kwam stellen met de welbekende woorden: heb je even een minuutje, wat vervolgens erin resulteerde dat ik vaak een half uur tot een uur later pas buitenstond wat kenmerkend is voor de tijd die je voor me nam. Ook al werkte ik op een compleet ander onderwerp dan de rest van de MCAT groep, het sparren met je heb ik altijd gewaardeerd. An en Ludwig, dank jullie wel voor alle input, begeleiding, peptalks als iets soms niet ging zoals ik het wou, de taalcorrecties en jullie vertrouwen in mij. Het gaf me de kans om me te ontwikkelen als wetenschapper en als mens.

De leden van de leescommissie zou ik graag via deze weg willen bedanken: Prof. dr. F.C.S. Ramaekers, Prof. dr. R.P. Coppes, Dr. P. Willems, Prof. dr. L.P.A.J. Schrauwen en Dr. F. van Tienen voor hun tijd om mijn proefschrift te lezen en te beoordelen.

I would also like to thank all my co-authors and collaborators for their contribution and scientific input.

Ralf and Katarina, hereby I would like to thank you both for being master-internship supervisors and now collaborators. The distance between Sweden and the Netherlands is not so far and I am really grateful we were still able to publish the GST paper. Your kind words and motivational speeches always put a smile on my face $(-)$. 
Guido, ooit was je mijn stagebegeleider en gelukkig werken we nog steeds af en toe samen. Dank je wel voor al je hulp bij de GSH metingen, je wetenschappelijke bijdrages, en voor het feit dat je deur altijd open staat. Ook wil ik graag Marie-José bedanken voor alle hulp bij de GSH meetingen.

Roland, ook Groningen - Maastricht lijkt een eind maar het valt goed te overbruggen via emails. Dank je wel voor je bijdrage aan de mtDNA depletie studies.

René and Rana, unfortunally the MNGIE project didn't worked out as we planned for my project but I am grateful for all the help and input you gave me and wish you all the best. Rana, good luck with finishing your PhD project.

Emmani, dank je wel voor alle tijd, aandacht en het helpen in tijd van nood, als de pH meter of de platereader het weer eens begaf. En natuurlijk ook bedankt voor het delen van je chocolade voorraad :;.

In het begin van mijn PhD traject mocht ik veel experimenten doen bij Klinische Genetica en ook via deze weg wil ik jullie graag bedanken. Bert, Florence, Erika, Alexandra, Romy, Tom, Auke, etc., bedankt voor het beantwoorden van mijn vragen en de gezellige praatjes op het lab.

Ook met Maastro-clinic heb ik aangenaam samengewerkt. Cary, Timo, Karen, Aniek en Bart; dank jullie wel voor de prettige samenwerking, input, gezellige meetings en emails.

As I am afraid that I will forget to thank you all, All Maastro-lab fellows and the previous lab members: thanks a lot for all the fun laughs at the Team buildings and the lab, and of course for all the scientific input. It were 5 awesome years!

Beste Carla, als ik aan jou denk moet ik gelijk denken aan de vensterbank waar ik op ging zitten als ik een vraag had. Dank je wel voor het altijd geduldig beantwoorden van mijn vragen en je vriendelijke woorden tijdens een kopje thee.

Technicians (Kim S., Hanneke, Jolanda, Lydie, Evelien, Rianne, Natasja and Kim P.) thanks for being our little lab elves.

Alle mCAT en oud-mCATers ook jullie bedankt. Ook al werkte ik op een ander onderwerp dan de meesten van jullie, toch dachten jullie altijd mee met het opzetten van experimenten, dank jullie wel!

Raymon, de stille kracht bij mCAT, behalve bij bepaalde nummers in de TC. Ik heb me altijd goed geamuseerd als we een duet zongen. Al denk ik wel dat sommige labmensen blij zijn dat dat niet meer voorkomt ;). We drinken snel weer eens een cola. Nicolle, we zijn ongeveer gelijk klaar met onze PhD, en je weet het 'gedeelde smart is halve smart'. Is het al tijd voor bier? Veronica and Damiënne, you are just at the beginning of your PhD but please take this advice from me: Please enjoy your PhD, it is over before you know it. Ala and Linda, our two U2 fans: Thank you for all your input. Ala, thanks a lot 
for helping with the stainings, you are a true magician when it comes to stainings. Rianne en Natasja, bedankt voor alle extra handjes die ik soms goed kon gebruiken, evenals voor het delen van goede recepten. Dank jullie wel voor alles. Natuurlijk wil ik ook mijn oud-studenten bedanken, niet alleen voor de data welke door jullie gegenereerd werden, maar ook omdat ik me dankzij jullie kon ontwikkelen als begeleider en als persoon. Rianne, Kelly, Vincent en Thara bedankt hiervoor.

Autophagy (Kasper, Kim S., Hanneke, Tom en Marijke) dank jullie wel voor het af en toe adopteren van me. Zo was dat mito eilandje toch niet zo heel erg klein en ver weg. Jullie gaven me altijd goede input en spraken (soms) wijze woorden ;). Dank jullie wel dat jullie deur altijd open stond. Hanneke, we hebben wat afgelachen samen op het lab en erbuiten. Gelukkig heb ik ooit met je de afspraak gemaakt dat wat buiten werktijd gebeurt buiten werktijd blijft. Scheelt toch weer wat fluorescerende golf foto's ;). Tom, mede-aio, de rust zelve, en mede koffiedrinker, altijd maakte je tijd voor een goed gesprek over wetenschap of andere zaken, dank je wel voor al je steun. Marijke, ook al zit je nog niet zo lang bij ons op het lab, toch dank je wel voor je bemoedigende woorden de laatste maanden. Kim S. met wie moet ik nu dansjes doen op jaren 80 nummers? Just keep on dancing!

Notch, Marc, Jan, Arjan, Carolien, Eloy, Venus, Lydie, Jolanda, Lorena and Jonathan.

Thanks a lot for all the input during MLM's and at the lab. Marc, bedankt voor je input en voor het overbrengen van je voorliefde voor de wetenschap. Jan, dank je wel voor de goede input bij het geven van presentaties, je weet echt als geen ander hoe een verhaal te vertellen. Ook wou ik je bedanken voor de twee keer dat ons team de Maastro teambuilding heeft gewonnen. Ik denk dat we door ons dansje wel bonuspunten hebben verdiend. Carolien, dank je wel voor je vriendelijke woorden en gezellige sushi etentjes. Venus, thank you for the nice dinners and company. Arjan, dank je wel voor het delen van al je kennis en de gezellige weekenden. Je was er altijd $;$; en gelukkig heb je me nooit laten schrikken, dank je wel! Notch, PhD students, thank you for all the laughs, bloopers and fun times we had. I know you are all getting involved in learning Dutch so maybe this is a good sentence to start with: een dag niet gelachen, is een dag niet geleefd. Good luck with your PhD!

Ook wil ik de oud-Maastro medewerkers bedanken: Frank, Caroline, Roger, Marco, Barry, Maud, Twan, Kranthi, Simon, Sarah en Sanaz. Ook jullie hebben bijgedragen aan de fijne tijd die ik heb gehad, terwijl ik rondliep op Maastro-Lab. Eerst op nivo 5 en later op de derde verdieping. Dank jullie wel!

Sanaz, thank you for the company during late nights, sweet words and for your help at our Rotterdamtrip. Sarah, mijn oud - kamergenootje: dank je wel voor alles! Het was erg leuk om samen met jou een kamer te delen. Kranthi, how is life after the PhD... often we dreamed about it and now it is becoming reality :) Thank you for joining me on the ride! Simon, het is altijd goed om met iemand je voorliefde voor panda's te delen, 
bedankt. Tevens wil ik je bedanken voor het geven van alle tips \& tricks gedurende het afronden van mijn PhD.

Maar natuurlijk zijn het niet mijn collega's alleen die ervoor hebben gezorgd dat mijn $\mathrm{PhD}$ een leuke en leerzame tijd was. Ook familie en vrienden, dank jullie wel voor alles.

De afdeling Boxtel: Karin, Rodger, Jurrie, Joyce en Eva. Eva dank je wel voor het voorstellen van mij aan Teun. Het wordt zeer gewaardeerd. Karin, Rodger, Jurrie en Joyce, Dank jullie wel voor jullie warme vriendschap en de gezellige avonden en promotieontwijkingsactiviteiten.

Annette, we were colleagues in Stockholm but we remained staying in touch. Every email, post-card or letter puts a smile on my face. Next time I am coming along with the Indian.

Anna, my dearest friend and cover designer. You live now on the other side of the globe. Did you really have to move so far away? You took me in your house in Stockholm, and from there our friendship grew. Thank you for always being there for me, even when it is at night time for you, or for me. You are a true friend! Stor kram!

Veer en Mo, wat een avonturen hebben we ondertussen al beleefd. Ooit begonnen als huisgenootjes, maar hier groeide een hechte vriendschap uit. De onderburen zijn vast blij dat we zijn verhuisd en dat ze niet meer naar de toppers in concert op volume 100 hoeven te luisteren. Jullie zijn er altijd voor me. Soms zien we elkaar wat minder (Mo waarom moest je nu ook helemaal naar Singapore verhuizen?) maar zodra we elkaar zien zit het direct weer goed. Dank jullie wel voor jullie warme vriendschap, toppers!

Marcelle en Jean: Jullie staan al mijn hele leven voor me klaar. Dank jullie wel voor alle goede zorgen en voor de interesse in mijn promotietraject.

Natuurlijk wil ik ook de familie van Teun bedanken: Sylvia, Joris, Dorien, Noélie, Han en Jacqueline. Sylvia, dank je wel dat je zo'n lief en zacht mens bent. Dank je wel voor je oprechtheid en betrokkenheid. Ook Joris, Dorien en Noélie wil ik graag via deze weg bedanken voor alle lieve knuffels, appjes en kaartjes. Noélie ik kom je snel weer knuffelen hoor. Han en Jacqueline, dank jullie wel voor de goede zorgen.

En als laatste wil ik mijn grootste motivators en persoonlijke cheerleaders bedanken.

Maarten, Mieke, Lotte en Eef. Maarten, je bent de grote broer tegen wie ik al mijn hele leven opkijk. Je zorgt ervoor dat ik het beste uit mijzelf weet te halen en weet ook goed te benadrukken wanneer ik even gas terug moet nemen. Je kunt als de beste dingen soms in zijn perspectief plaatsen, maar dat neemt niet weg dat je er altijd voor mij bent. Gelukkig zorg je samen met Mieke vaak voor de nodige afleiding. Mieke, dank je wel voor al je betrokkenheid, fijne gesprekken over iets anders dan werk, en dat jullie deur altijd open staat. Lotte en Eef, mijn lieve nichtjes, op dit moment begrijpen jullie nog niet heel veel van dit alles, maar toch hebben ook jullie bijgedragen aan mijn goede 
humeur tijdens mijn PhD. De filmpjes die jullie ouders maakten als jullie weer een mijlpaal bereikten of iets geks/stouts deden toverde vaak een grote glimlach op mijn gezicht. Dank jullie wel hiervoor! Dikke knuffel!

Papa en mama. Echt geen woord kan beschrijven hoe zeer ik jullie wil bedanken. Degene die altijd in mij geloofd hebben waren jullie. Elke studie, en elk vervolgstap van mijn studie zagen jullie soms met angst tegemoet. Het feit dat ik altijd een uitdaging zocht en me niet wou laten definiëren door die stomme dyslexie zorgde ervoor dat ik altijd het beste uit mezelf kon halen met de veiligheid dat ik wist dat het goed was als het niet zou lukken. Papa, dank je wel dat je zelfs nu nog altijd alles voor me nakijkt als ik het vraag. Je leest mijn (gepubliceerde) artikelen en zoekt zelfs onbekende terminologie op zodat je het begrijpt. Je fameuze zin, dat zoeken we even op staat voor altijd in mijn geheugen gegrift. Ook was jij degene die mijn interesse in wetenschap, al vroeg aanwakkerde door altijd geduldig en uitvoerig over allerlei natuurverschijnselen te vertellen. Je bent zelf geen wetenschapper geworden maar je had er zo een kunnen zijn (:). Mama, de doorzetter van het stel. Je bent de grootste doorzetter die ik ken. Dank je wel dat je me al vroeg heb geleerd hebt, dat alles kan als je er maar in gelooft en dat je (bijna) alles kan leren. Ondanks dat je zelf een harde werker bent, ben je lief, erg betrokken en maak je nog altijd veel tijd voor me vrij. Gelukkig dacht je vaak mee naar oplossingen, wat soms zorgde voor een nachtelijke Whatsapp conversatie. Maar dank jullie wel voor jullie rotsvaste vertrouwen in mij en alle steun die jullie me geven. Deze is voor jullie!

Teun, we leerden elkaar al na 6 maanden kennen, hetgeen ervoor zorgde dat je gelijk werd meegezogen in de achtbaan die een PhD traject heet. Gedurende mijn hele PhD periode was je ervoor mij. Je verhuisde zelfs van Brabant naar Limburg, omdat het anders niet ging werken, omdat mijn cellijnen ook in het weekend vaak wat liefde nodig hadden. Zelf heb je het niet altijd gemakkelijk gehad, maar ik ben onwijs trots hoe het nu met je gaat. Je bent er altijd voor me en hebt me vaak beschermd voor alle randzaken zodat ik me kon concentreren op mijn PhD. Je bent een topper, echt, beloofd, pinkie promise. Samen kunnen we de wereld aan. 


\section{Curriculum Vitae}

Marike van Gisbergen was born on December $12^{\text {th }}, 1984$ in Geleen, the Netherlands. She was raised in a small village, Bunde, situated in the south of the Netherlands. Here, she spent her childhood and went to primary school. After this she started her high school at the Sint Maartens College in Maastricht and graduated in 2003 from the HAVO. In 2004, she obtained her propaedeutic in Applied Psychology from the Fontys University, Eindhoven, the Netherlands. In the following year, she obtained her propaedeutic in Biology and Medical Laboratory Research, University of Applied Sciences Utrecht, the Netherlands. She continued her bachelor studies at the Zuyd University, Heerlen, the Netherlands, and obtained her bachelor of applied Sciences in Biology and Medical Laboratory Research - biomedical and biochemical sciences in 2008. After obtaining her bachelor degree she started in 2009 with the Master in Molecular Life Sciences; Molecular Health sciences at the University of Maastricht. During her master studies, she performed her first internship at the department of Pharmacology and Toxicology at the same university. Here she investigated the reversibility of MonoHER (7-mono-O-( $\beta$-hydroxyethyl)rutoside) adduct formation with thiols. For her second internship, she went abroad to Scandinavia. In the group of prof. dr. Ralf Morgenstern in the Division of Biochemical Toxicology (Institute of Environmental Medicine) at the Karolinska Institute in Stockholm, Sweden, she characterized newly developed thiol reactive cytostatic drugs in relationship to glutathione transferase overexpression. In 2011, she obtained her Master degree and she continued to work on the same topic for a short period. In January 2012 Marike started her PhD studies at the department of Radiation Oncology (MaastRO lab) at the University of Maastricht. Here her research involved studying the role of mitochondria in a personalized cancer treatment approach. The results of her research are presented in this thesis. 



\section{List of publications}


1. M.W. van Gisbergen, K. Offermans, A.M. Voets, N.G. Lieuwes, R. Biemans, R.F. Hoffmann, L. Dubois*, P. Lambin*, Mitochondrial dysfunction inhibits hypoxiainduced HIF-1 $\alpha$ stabilization and expression of downstream targets. In preparation

2. M.W. van Gisbergen, A.M. Voets, R. Biemans, R.F. Hoffmann, M.J. Drittij-Reijnders, G.R.M.M. Haenen, I.H. Heijink, K.M.A. Rouschop, L. Dubois*, P. Lambin*, Distinct radiation responses after in vitro mtDNA depletion are potentially related to oxidative stress. Submitted

3. E.B.M. Nascimento, L.M. Sparks, A. Divoux, M.W. van Gisbergen, E.P.M. Broeders, J.A. Jörgensen, G. Schaart, N.D. Bouvy, W.D. van Marken Lichtenbelt, P. Schrauwen, Genetic markers of brown adipose tissue and in vitro brown adipose tissue activity in humans. Submitted

4. B. Reymen*, M.W. van Gisbergen*, A. J.G. Even, C. M.L. Zegers, M. Das, E. Vegt, J. E. Wilberger, F. Mottaghy, A. Yaromina, W. van Elmpt, D. De Ruysscher, L. Dubois, P. Lambin, Nitroglycerin's influence on tumor hypoxia and perfusion in non-small cell lung cancer: a window-of opportunity trial. Submitted

5. R. Yadak, P.S. Smitt, M.W. van Gisbergen, N.P. van Til, I.F. de Coo, Mitochondrial Neurogastrointestinal Encephalomyopathy: From Pathogenesis to Emerging Therapeutic Options. Frontiers in Cellular Neuroscience, 2017. 11(31). doi: 10.3389/fncel.2017.00031.

6. S.J. van Kuijk*, N.K. Parvathaneni*, R. Niemans*, M.W. van Gisbergen*, F. Carta, D. Vullo, S. Pastorekova, A. Yaromina, C.T. Supuran, L.J. Dubois**, J.Y. Winum**, and P. Lambin**, New approach of delivering cytotoxic drugs towards CAIX expressing cells: A concept of dual-target drugs. European journal of medicinal chemistry, 2017. 127: p. 691-702. doi: 10.1016/j.ejmech.2016.10.037.

7. P. Lambin, J. Zindler, B.G. Vanneste, L.V. De Voorde, D. Eekers, I. Compter, K.M. Panth, J. Peerlings, R.T. Larue, T.M. Deist, A. Jochems, T. Lustberg, J. van Soest, E.E. de Jong, A.J. Even, B. Reymen, N. Rekers, M. van Gisbergen, E. Roelofs, S. Carvalho, R.T. Leijenaar, C.M. Zegers, M. Jacobs, J. van Timmeren, P. Brouwers, J.A. Lal, L. Dubois, A. Yaromina, E.J. Van Limbergen, M. Berbee, W. van Elmpt, C. Oberije, B. Ramaekers, A. Dekker, L.J. Boersma, F. Hoebers, K.M. Smits, A.J. Berlanga, and S. Walsh, Decision support systems for personalized and participative radiation oncology. Advanced drug delivery reviews, 2017. 109: p. 131-153. doi: 10.1016/j.addr.2016.01.006. 
8. M.W. van Gisbergen*, M. Cebula*, J. Zhang, A. Ottosson-Wadlund, L. Dubois, P. Lambin, K.D. Tew, D.M. Townsend, G.R. Haenen, M.J. Drittij-Reijnders, H. Saneyoshi, M. Araki, Y. Shishido, Y. Ito, E.S. Arner, H. Abe, R. Morgenstern, and K. Johansson, Chemical Reactivity Window Determines Prodrug Efficiency toward Glutathione Transferase Overexpressing Cancer Cells. Molecular pharmaceutics, 2016. 13(6): p. 2010-25. doi: 10.1021/acs.molpharmaceut.6b00140.

9. M.W. van Gisbergen, A.M. Voets, M.H. Starmans, I.F. de Coo, R. Yadak, R.F. Hoffmann, P.C. Boutros, H.J. Smeets, L. Dubois, and P. Lambin, How do changes in the mtDNA and mitochondrial dysfunction influence cancer and cancer therapy? Challenges, opportunities and models. Mutation research. Reviews in mutation research, 2015. 764: p. 16-30. doi: 10.1016/j.mrrev.2015.01.001.

10. L.J. Dubois, R. Niemans, S.J. van Kuijk, K.M. Panth, N.K. Parvathaneni, S.G. Peeters, C.M. Zegers, N.H. Rekers, M.W. van Gisbergen, R. Biemans, N.G. Lieuwes, L. Spiegelberg, A. Yaromina, J.Y. Winum, M. Vooijs, and P. Lambin, New ways to image and target tumour hypoxia and its molecular responses. Radiotherapy and oncology: journal of the European Society for Therapeutic Radiology and Oncology, 2015. 116(3): p. 352-7. doi: 10.1016/j.radonc.2015.08.022.

11. H. Jacobs, M. Moalin, M.W. van Gisbergen, A. Bast, W.J. van der Vijgh, and G.R. Haenen, An essential difference in the reactivity of the glutathione adducts of the structurally closely related flavonoids monoHER and quercetin. Free radical biology \& medicine, 2011. 51(11): p. 2118-23. doi: 10.1016/j.freeradbiomed.2011.09.013.

*Indicates equal contribution

** Indicates equal contribution 